\title{
Protein thiol oxidoreductas and allergic airways disease
}

Citation for published version (APA):

Hoffman, S. M. (2016). Protein thiol oxidoreductas and allergic airways disease. [Doctoral Thesis, Maastricht University]. https://doi.org/10.26481/dis.20160302sh

Document status and date:

Published: 01/01/2016

DOI:

10.26481/dis.20160302sh

Document Version:

Publisher's PDF, also known as Version of record

\section{Please check the document version of this publication:}

- A submitted manuscript is the version of the article upon submission and before peer-review. There can be important differences between the submitted version and the official published version of record.

People interested in the research are advised to contact the author for the final version of the publication, or visit the DOI to the publisher's website.

- The final author version and the galley proof are versions of the publication after peer review.

- The final published version features the final layout of the paper including the volume, issue and page numbers.

Link to publication

\footnotetext{
General rights rights.

- You may freely distribute the URL identifying the publication in the public portal. please follow below link for the End User Agreement:

www.umlib.nl/taverne-license

Take down policy

If you believe that this document breaches copyright please contact us at:

repository@maastrichtuniversity.nl

providing details and we will investigate your claim.
}

Copyright and moral rights for the publications made accessible in the public portal are retained by the authors and/or other copyright owners and it is a condition of accessing publications that users recognise and abide by the legal requirements associated with these

- Users may download and print one copy of any publication from the public portal for the purpose of private study or research.

- You may not further distribute the material or use it for any profit-making activity or commercial gain

If the publication is distributed under the terms of Article $25 \mathrm{fa}$ of the Dutch Copyright Act, indicated by the "Taverne" license above, 


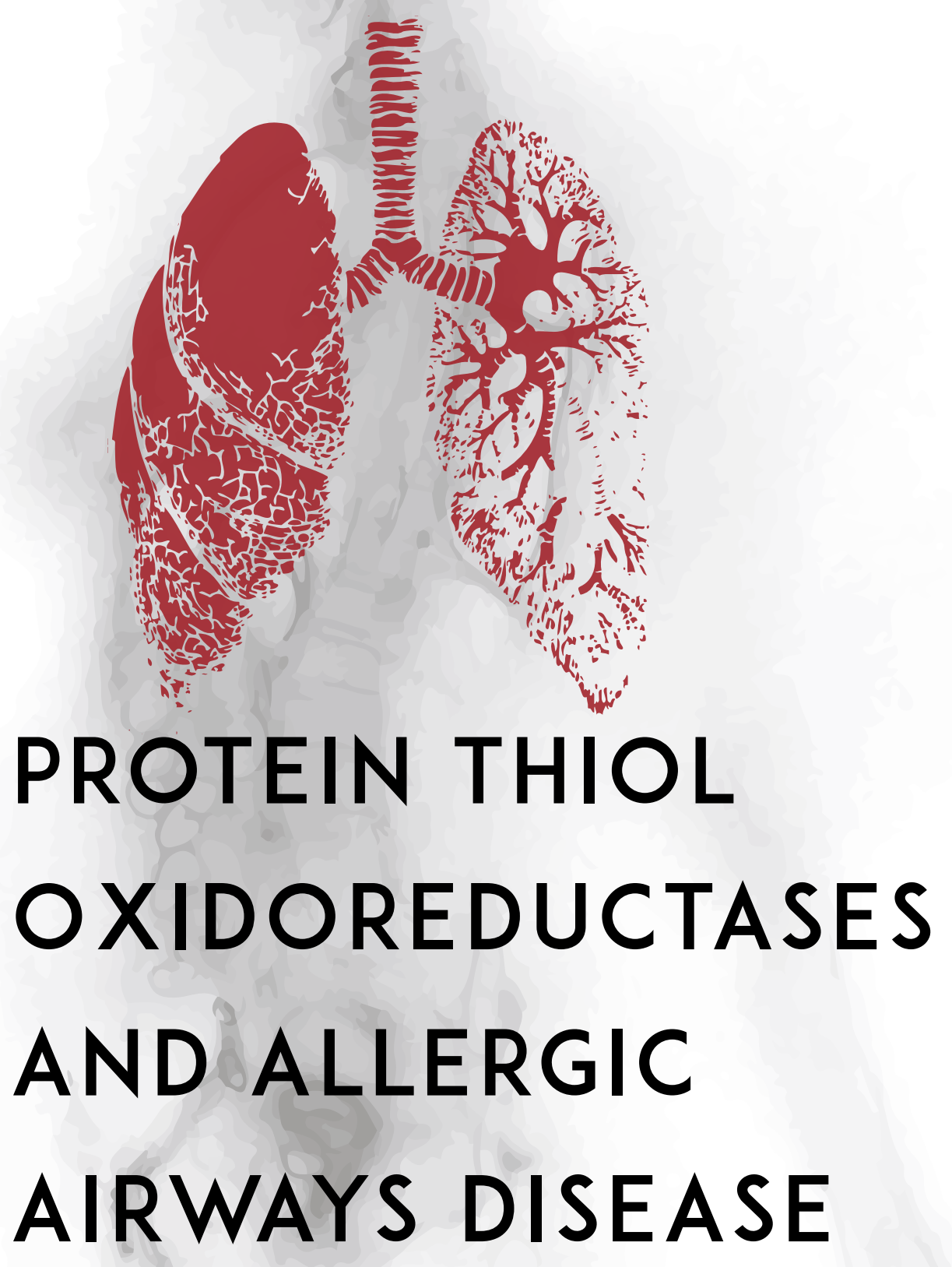

Sidra M. Hoffman 
(C) Copyright, Sidra M. Hoffman

Cover Design: VanBrunt, Ashley

Print: Datawyse | Universitaire Pers Maastricht ISBN: 9789461595416 


\title{
Protein thiol oxidoreductases and allergic airways disease
}

\author{
DISSERTATION \\ To obtain the degree of doctor at Maastricht University \\ on the authority of Rector Magnificus, Prof. dr. L.L.G. Soete \\ in accordance with the decision on the Board of Deans, \\ to be defended in public on Wednesday March 2, 2016 at 14:00 hours \\ by
}

Sidra M Hoffman 


\section{Promoter}

Prof. dr. E.F.M. Wouters

Prof. dr. Y.M.W. Janssen-Heininger (University of Vermont)

\section{Copromoter}

Dr. V. Anathy (University of Vermont)

\section{Committee}

Prof. dr. E. Dompeling, Chairman

Prof. dr. A. Bast

Prof. dr. G. Brusselle (Ghent University)

Prof. dr. B.W.W. Kramer

Prof. dr. R. Louis (University of Liège) 


\section{Contents}

1: General Introduction $\quad 7$

2: $\quad$ Endoplasmic reticulum stress mediates house dust mite-induced airway epithelial $\quad 39$ apoptosis and fibrosis

3: Epithelial NF-KB orchestrates house dust mite-induced airway inflammation, hyperresponsiveness, and fibrotic remodeling

4: $\quad$ Genetic ablation of glutaredoxin-1 causes enhanced resolution of enhanced airways hyperresponsiveness and mucus metaplasia in mice with allergic airways disease

5: Ablation of glutaredoxin-1 modulates airway neutrophilia, IL-17A and airways hyperresponsiveness in house dust mite-induced allergic airways disease

6: Summary \& Future Directions

7: $\quad$ Valorisation

Dankwoord

Curriculum Vitae

List of Publications 



\section{CHAPTER 1}

General Introduction 


\section{Asthma}

Asthma is a disorder characterized by chronic airway inflammation, mucus secreting cell hyperplasia and metaplasia, airway hyperreactivity (AHR) and airway remodeling [1]. The prevalence of asthma has increased dramatically over the past two decades, affecting 300 million people globally. As this number grows, the demand for therapeutic intervention also increases, and hundreds of molecules are currently in development [2]. Current therapeutics, however, are of limited efficacy as will be discussed further in this review.

The etiology of asthma is complex and multifactorial. Clinical manifestations include allergic asthma, severe and steroid-resistant asthma, as well as exacerbations induced by air pollution, cigarette smoke, obesity and exercise-associated, or even aspirin-induced asthma [3, 4]. The cause(s) of asthma remain incompletely understood, but it is believed that the complex interplay between genetic and environmental factors becomes unbalanced and predisposes individuals to hypersensitive reactions. In the mid-1980's, type 2 CD4+ lymphocytes ( $T_{H} 2$ cells) were thought to be integral to the pathogenesis of allergic airway disease based on the observation that specific $T_{H} 2$ subtypes existed in asthmatic patients [1]. These T cells were able to secrete cytokines (IL-3, -4, -5, -9, -13 and GM-CSF) responsible for the recruitment, priming and survival of the primary effector cells (e.g., mast cells and eosinophils) [5]. $\mathrm{T}_{\mathrm{H}} 2-$ type cytokines are now known to contribute to tissue damage, mucus metaplasia, airway obstruction and chronic inflammation of the airways; all hallmarks of persistent asthma [6]. Antigen-presenting dendritic cells (DCS) were also identified in individuals susceptible to indoor and outdoor environmental allergens [7]. In the last decade, studies have been aimed at addressing how specific DC subsets recognize allergens and communicate with naive T cells through Class II MHC T cell receptor (CD3) interaction and engagement of co-stimulatory molecules [5]. Based upon these collective studies, among the potential new targets being evaluated for treatment of asthma are the immune-stimulating cytokines IL-4 and IL-13. These cytokines are critical to asthma pathophysiology, and may hold promise in the treatment of specific patient populations [8].

Patients suffering from allergic airway disease often exhibit increased serum IgE levels and predominant eosinophilic infiltration [9] and eosinophilic driven chemokines CCL-11 and -24 within the airways. The discovery that administration of a monoclonal antibody targeting FcepsilonR1, the high affinity IgE binding site on mast cells, blocks mast cell activation and degranulation [10], led to clinical trials using the monoclonal antibody, Omalizumab. Omalizumab led to an almost total inhibition of the early and late asthmatic responses to inhaled allergen. However, only one third to one half of patients with severe allergic asthma appeared to respond to Omalizumab, potentially corresponding to the extent 
that blockade of IgE binding to mast cells and dendritic cells produce down-regulation of FcER1 [5]. More recently, multiple studies have investigated the ability of Mepolizumab, a humanized monoclonal antibody against interleukin-5 (IL-5) to selectively inhibit eosinophilic inflammation, reduce the number of eosinophils in both sputum and blood, and reduce the frequency of exacerbations and the need for treatment with systemic glucocorticoids in patients with severe asthma and persistent eosinophilic inflammation. Following a trial evaluating dose ranging efficacy and safety in response to intravenous Mepolizumab [11], a newly reported study utilizing the same defining characteristics (blood eosinophil count, number of previous exacerbations and dose of inhaled corticosteroids) evaluated subcutaneous versus intravenous administration of Mepolizumab to determine whether the use of anti-IL-5 therapy would mitigate the requirement for frequent glucocorticoid use in patients with severe asthma. The occurrence of exacerbation was significantly lessened in those patients receiving both intravenous and subcutaneous Mepolizumab and was associated with improvements in markers of asthma control [12].

Recent clinical and experimental observations have suggested however, that asthma is much more heterogeneous than proposed by the $T_{H} 2$ paradigm, and roles for $T_{H} 1$ cells, regulatory $T\left(T_{\text {Reg }}\right)$ cells, $T_{H} 17$, and innate lymphoid cells (ILC) have been identified. While it is known that multiple $T$ cell lineages contribute to the pathogenesis of allergic airway disease, the extent and degree to which these cells orchestrate the respective pheno(endo)types of human asthma remains unknown. Non- $T_{H} 2$ responses, such as IL-17A, E, or F production are frequently found in the lungs of asthma patients, particularly those with severe or corticosteroid-resistant asthma [3]. IL-17E, released by alveolar macrophages, is associated with $T_{H} 1$ and $T_{H} 17$ immunity and may be involved in the mixed T-cell response [1]. IL-17 and IL-22 have been shown to sustain inflammation in allergic diseases and affect a variety of cell types, particularly epithelial cells in the inflamed tissues [13]. Abundant neutrophilic infiltration may also play a role in asthma pathophysiology possibly through release of ROS, RNS, cytokines, lipid mediators and enzymes including elastase, myeloperoxidases and cathepsin G [14]. Notably, the presence of neutrophils in lungs of severe asthmatics has been linked to IL-17 production, and is associated with steroid resistance [15-18].

Originally described as $\mathrm{T}_{H} 2$ cytokine producing non-B/non-T cells, Innate Lymphoid Cells (ILCS) were identified by multiple groups in 2010. One study identified c-Kit, Sca-1, IL-17R and IL-33-expressing 'natural helper' cells, distinct from lymphoid progenitors in adipose tissue of the peritoneal cavity in response to IL-2, important in the protection against helminth infection [19]. Similar observations were reported characterizing a distinct population of 'nuocytes' in the mesenteric lymph nodes expanded in vivo in response to IL-25 and IL-33, and were an early source of IL-13 during helminth infection with $N$. 
brasiliensis [20], and additional studies also identified these cells in the spleen and liver, and labeled them 'innate helper type 2 cells' [21]. Despite subtle differences reported among natural helper cells, nuocytes and innate helper type 2 cells [22], these cells are now categorized as group 2 innate lymphoid cells (ILC2s). Specific functions for ILC subsets have been identified, as ILC2 cells expressing GATA3 and IL-33R are implicated in allergen-induced asthma [23, 24], while ILC3 cells are speculated to contribute to obesity-induced asthma [25]. ILC2s produce $\mathrm{T}_{\mathrm{H}} 2$ cytokines and are stimulated by IL-25 and IL-33 release [3] and recent studies in wild-type (WT) mice showed expansion of the ILC2 population in the lung and bronchoalveolar lavage fluid in a model of house dust mite (HDM)-induced allergic asthma, and were a major source of IL-5 and IL-13 [26]. Additionally, in vivo adoptive transfer experiments demonstrated that IL-13 produced by ILC2s was sufficient to mediate IL-33-induced airway inflammation [21, 27, 28]. ILC2s may therefore be critical mediators of allergic airway inflammation in the lung.

In addition to deregulation of the immune response, there is evolving awareness of the active participation of structural elements, such as the airway epithelium, airway smooth muscle, and endothelium in asthma, especially as the disease progresses to a more severe and chronic phenotype [5]. Hallmark pathological features of asthma, such as remodeling of the epithelium, are thought to induce the clinical symptoms of the disease: airway obstruction, coughing, dyspnea, and wheezing [5]. Inhaled corticosteroids and combination therapies with long-acting $\beta$-agonists are the mainstay pharmacotherapies for controlling the symptoms of asthma. Although several more immunotherapies are currently in development as described above (IgE mAb, IL-5 mAb, and IL-4 mAb) [29], none of these treatments can cure established asthma. Therefore, further elucidation of the biochemical processes that culminate in the diverse phenotypes of human asthma, and the contribution of immune and structural cells herein are paramount toward the identification of new therapeutic targets.

\subsection{Airway epithelium}

Epithelial cells are the primary component of tissues lining the surfaces of organs or internal cavities and thus, are involved in a plethora of physiological processes. Until recently, it was believed that the primary contribution of epithelial cells to host protection was a result of physicochemical effector functions and, in the case of epithelial barrier disruption or pathogen invasion, a systemic immune response would be invoked by direct microbial challenge [30]. This concept is being amended based on growing evidence from mouse models and is widely accepted that epithelial cell dysregulation can be a primary cause of inflammatory pathology in various tissues. The direct response of epithelial cells to 
infection and/or stress can strongly influence dendritic cell action and subsequent regulation of adaptive immune responses [30].

Multiple mechanisms are used to regulate immune homeostasis in epithelial tissues. Integrating microbial and other stress inputs into immune regulatory systems ensures the maintenance of a healthy immune balance and host defense, while preventing excessive and potentially dangerous inflammatory responses. These regulatory mechanisms prevent disease pathogenesis in most healthy individuals. However, deregulation of immune homeostasis in respective tissues is believed to cause chronic inflammatory disease in a significant number of patients [31].

The airway epithelium provides a physical barrier between host and environment that protects from injurious and infectious stimuli that gain access to the respiratory tract through inspiration or aspiration. Well-characterized functions of the airway epithelium include mechanical clearance of offending agents and production of antimicrobial agents [32]. Furthermore, airway epithelial cells function critically to coordinate innate and adaptive immune responses. Airway epithelial cells express a number of Toll-like receptors (TLRs), and local and systemic inflammation results in prominent activation of the nuclear factor-kappa B (NF-KB) pathway in these cells, which is sufficient to generate lung injury [33]. In patients with chronic asthma, long-term remodeling of the lung has been observed, resulting in thickening of the basement membranes within the lung and conducting airways, mucus secreting cell hyperplasia, smooth muscle proliferation, increased fibrosis and decreased lung capacity [34].

When functioning normally, the airway epithelium forms a physical barrier maintained by the formation of tight junctions. Disruption of tissue homeostasis and injury/ repair mechanisms in allergictype inflammation has been implicated in impaired barrier function within the airway epithelium. This allows inhaled substances to pass more easily into the airway and interact with immune and inflammatory cells [35]. The regulation of oxidants generated by the exposure of the airways to a torrent of chemical, particulate and/or biological insults is also impaired in asthma. Enhanced production of oxidant, coupled to defective antioxidant defenses, that include glutathione peroxidase, superoxide dismutase or catalase [36], collectively may contribute to altered homeostatic function of epithelial cells. Strong evidence exists to support the structural and functional role of the airway epithelium in asthma, and as will be discussed in the next paragraph, activation of a cardinal pathway in epithelial cells that plays a critical role in the pathogenesis of asthma. 


\subsection{Endoplasmic reticulum stress}

The endoplasmic reticulum (ER) is a membranous network of branching tubules and sacs that extends throughout the cytoplasm of the cell, adjoining the nuclear envelope [37]. The ER is primarily recognized for protein biosynthesis, folding, assembly and modification, notably glycosylation and disulfide bond formation of numerous soluble and membrane proteins [38, 39]. In comparison to the reducing environment of the cytosol where molar amounts of glutathione (GSH) may exceed that of glutathione disulfide (GSSG) by 100-fold, a highly oxidizing environment exists within the ER (GSH:GSSG 3:1) to promote protein disulfide bond formation [39]. The ER also functions as a dynamic calcium store, which responds to growth factors, hormones, and stimuli that perturb cellular energy levels, nutrient availability or redox status [37]. Evidence suggests that the ER is a key site where intracellular signals mediated by these factors are sensed, integrated, and transmitted, allowing coordination of downstream responses. Recently, it has been shown that the ER mediates a specific set of intracellular signaling pathways in response to the accumulation of unfolded or misfolded proteins. It is known that physiological states that increase the demand for protein folding, or stimuli that disrupt the reactions by which proteins are posttranslationally modified to form their final conformation, create an increased protein folding load beyond the capacity of the ER. This causes unfolded or misfolded proteins to accumulate in the ER lumen- a condition referred to as ER stress. Studies of the UPR have broadened the understanding of the mechanisms by which inflammation can be initiated [37].

In mammalian cells, the main UPR signaling cascades are initiated by three ER-localized protein sensors: protein kinase-like ER kinase (PERK), activating transcription factor 6 (ATF6), and inositolrequiring $1 \alpha(\operatorname{IRE} 1 \alpha)$. Under normal physiological conditions, these proteins are held inactive by their interaction with the ER chaperone BiP (GRP78); however, during ER stress, BiP preferentially binds unfolded or misfolded proteins resulting in the activation of ER stress proteins, inhibition of protein synthesis, increased expression of ER-resident chaperones, and degradation of terminally misfolded proteins [37]. Following its homodimerization and trans-autophosphorylation, PERK functions to phosphorylate the $\alpha$-subunit of eukaryotic translation-initiation factor $2 \alpha$ (elF $2 \alpha$ ) [40, 41]. This results in the inhibition of the assembly of the $80 \mathrm{~S}$ ribosome and consequently, protein synthesis. Phosphorylation of elF2 $\alpha$ also results in the preferential translation of the transcription factor ATF4 [42, 43], inducing the expression of UPR target genes involved in amino-acid biosynthesis and transport, the oxidative stress response, and ER-stress-induced apoptosis [44]. Activation of IRE1 $\alpha$ via autophosphorylation allows targeting of the ubiquitously expressed XBP1 mRNA, in which the nuclease activity of IRE1 $\alpha$ removes a 26-base intron, triggering translation of the XBP1 transcription factor. Active XBP1 translocates to the 
nucleus initiating the expression of UPR target genes $[45,46]$. In parallel with XBP1, cleaved ATF6 functions to induce transcription of genes encoding ER chaperones and enzymes that promote protein folding, maturation, secretion and ER-associated protein degradation [47]. However, if the cell ultimately fails to resolve the protein-folding defect and restore homeostasis in the ER, the UPR will evoke apoptotic pathways to protect the organism by removing the stressed cells that produce misfolded or malfunctioning proteins $[45,46]$.

Downstream of the PERK- elF2 $\alpha$ - ATF4 and ATF6 pathways, ER-stress-induced apoptosis is largely mediated by CCAAT/-enhancer-binding protein homologous protein (CHOP). Using human and mouse cell culture and in vivo models of ER stress-induced apoptosis, it has recently been shown that cytosolic calcium induces expression of the Fas death receptor through calcium/calmodulin- protein kinase II, and c-Jun N-terminal kinase (JNK) [48]. IRE1 can also interact with tumor-necrosis factor- $\alpha$ (TNF $\alpha$ )-receptorassociated factor 2 (TRAF2), which can function pro-apoptotically through association with apoptosis signal-regulating kinase 1 (ASK1) and JNK [39]. It has also been suggested that chronic activation of ATF6 $\alpha$ can lead to the induction of CHOP and subsequent up regulation of proapototic Bak-mediated activation of caspases and apoptosis [49-52].

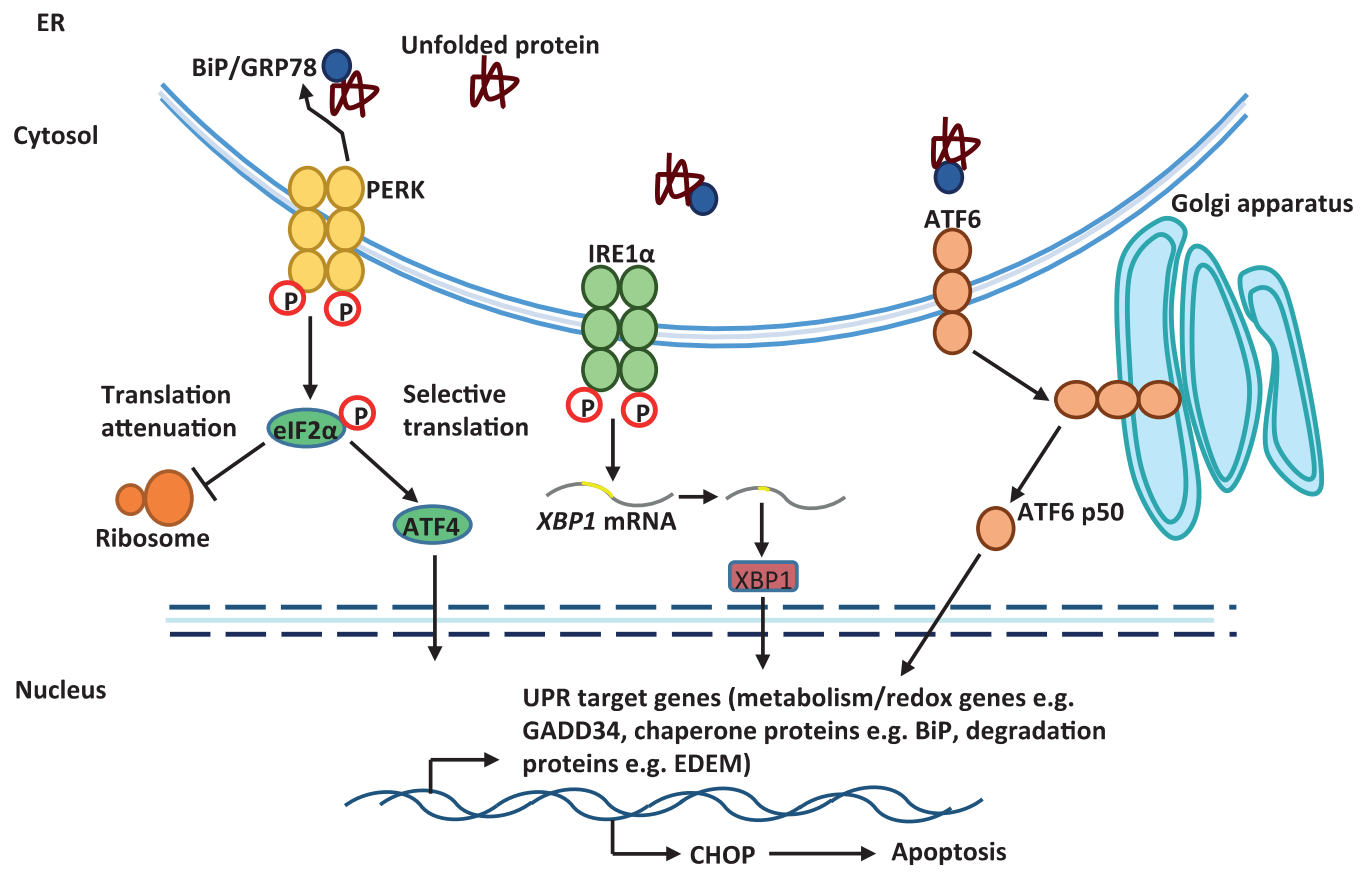

Figure 1. Endoplasmic reticulum stress and the UPR. During conditions of ER stress, BiP preferentially binds unfolded or misfolded proteins resulting in the activation of PERK, IRE1 $\alpha$ and ATF6: initiators of the three main 
signaling cascades of the UPR. PERK mediates general repression of mRNA translation by phosphorylation of elF $2 \alpha$ however, concomitant selective translation of ATF4 activates transcription of UPR target genes. IRE1 $\alpha$ dimerization triggers its endoribonuclease activity to induce cleavage of $x b p 1 \mathrm{mRNA}$, which is ultimately translated to produce XBP1. ATF6 released from BiP translocates to the golgi where it is cleaved, yielding an active cytosolic fragment (ATF6 p50). Cleaved ATF6 acts with XBP1 to induce expression of genes encoding UPR target genes.

\section{PDIs and oxidative protein folding}

There is accumulating evidence to suggest that protein misfolding and production of reactive oxygen species (ROS) in the ER are closely linked. The process of protein folding in the ER not only requires energy but also oxidizing conditions for intramolecular and intermolecular disulfide bond formation [53]. ER-resident enzymes including protein disulfide isomerase (PDI), ERp57, ERp72, PDIR, PDIp, P5 and oxidoreductin 1 (ERO1) are responsible for electron transport during disulfide bond formation [53, 54]. PDI directly accepts electrons from protein folding substrates, resulting in the oxidation of cysteine residues and the formation of disulfide bonds. ERO1 then uses a flavin-dependent reaction to transfer electrons from PDI to molecular oxygen, thereby oxidizing PDI. Hydrogen peroxide is produced as a byproduct of this process, and it has been estimated that approximately $25 \%$ of ROS generated in a cell is a consequence of disulfide bond formation in the ER during oxidative protein folding [53]. Furthermore, additional oxidative stress can result from the depletion of reduced GSH during reactions that reduce unstable and improperly formed disulfide bonds [55]. Importantly, cells have evolved mechanisms to limit the accumulation of ROS in response to ER stress. Following PERK activation and phosphorylation, nuclear factor-erythroid-derived 2 (NF-E2)-related factor 2 (NRF2) translocates to the nucleus and initiates transcription of a set of antioxidant and oxidant-detoxifying enzymes including $\mathrm{NAD}(\mathrm{P}) \mathrm{H}$-quinone oxidoreductase, haem oxygenase 1 and glutathione $S$-transferase $[56,57]$. Additionally, NRF2 and ATF4 both induce the transcription of genes involved in the maintenance of glutathione, an essential redox buffer in the cell $[44,58,59]$.

However, posttranslational modifications that alter PDI function have been recently described. Nitrosylation of cysteine residues in the active site of PDI has been observed in the brain tissue of patients with sporadic Parkinson's and Alzheimer's disease [60], both of which involve ER stress and UPR activation [39]. Additionally, PDI undergoes S-glutathionylation upon treatment with the anti-cancer agent PABA/NO [61], leading to enzyme inactivation, activation of the UPR, and cancer cell death [39]. Disturbance of redox homeostasis in the ER is an almost universal consequence of various human pathologies affecting the organelle, and collectively these studies provide evidence that redox-regulation of PDI is an upstream signaling event in the UPR. 
Previous studies have shown that several PDI members possess potential proapoptotic functions, and recently, more evidence of proapoptotic activities has emerged. Cell biological and biochemical studies have revealed ERp57 to be a component of the calnexin/calreticulin chaperone system that promotes the folding and quality control of glycoproteins in the ER [62]. The overall sequence identity at the amino acid level between ERp57 and PDI is 33\% [63], making ERp57 the closest known homolog of PDI. ERp57 is a versatile redox enzyme comprised of four thioredoxin-like domains, two of which are redox-active and contain a CGHC motif, whereas the other two domains are redox-inactive and are devoid of CXXC motifs [62]. In human endothelial cells, reducing ERp57 expression inhibited hyperoxiaor tunicamycin-induced apoptosis by blocking caspase-3 activation and Bip/GRP78 induction [64]. Our laboratory has demonstrated that decreasing or inhibiting ERp57 and glutathione S-transferase pi 1 (GSTP1) reduced oxidative processing and S-glutathionylation of Fas, resulting in cell survival (to be discussed in Chapter 6) [65]. Moreover, we have previously shown that decreasing ERp57 expression attenuates influenza A virus burden and reduces subsequent caspase-12 activation and apoptosis in epithelial cells [66]. More recently, studies including work from our laboratory on ER stress-mediated apoptosis, has shown involvement of ERp57 in disulfide-mediated oligomerization of proapoptotic Bak on the ER and mitochondria associated membranes [52, 67-69].

\section{Endoplasmic reticulum stress and asthma}

In recent years, ER stress has been implicated in the pathogenesis of numerous inflammatory disorders, and moreover, accumulating evidence suggests prolonged ER stress and UPR in the development and progression of chronic lung diseases including cystic fibrosis, $\alpha 1$-antitrypsin deficiency, idiopathic pulmonary fibrosis, pulmonary hypertension, chronic obstructive pulmonary disorders (COPD), and bronchial asthma [70]. Kim et al. demonstrated that ER stress markers GRP78 and CHOP were increased in peripheral blood mononuclear cells, and significantly elevated in bronchoalveolar lavage (BAL) fluid from asthmatic patients compared to healthy subjects [71]. Furthermore, an increase in the ER protein-folding load has been shown to result in the activation of NF-KB, a central player in inflammation and immunity [72, 73]. Inhibition of ER stress using 4-phenylbutyric acid (4-PBA) in an Ovalbumin (OVA)-LPS murine model of allergic airways disease attenuated both neutrophilic and eosinophilic inflammation and airways hyperresponsiveness through the inhibition of nuclear translocation of NF-KB [71]. Previous studies have also shown that ER stress leads to the UPR in airway epithelial cells [74, 75]. Findings by Martino et al. demonstrated that the mechanism of OVA- or IL-13induced mucin overproduction in airways is in part mediated by IRE1ß-dependent activation of XBP-1, and XBP1-dependent transcription of anterior gradient homolog 2 (AGR2), a gene implicated in airway 
and intestinal epithelial mucin production [76]. These reports however, did not address the implications of ER stress in other facets of asthma, such as subepithelial fibrosis, a feature of airway remodeling. Our group recently demonstrated induction of severe ER stress in human nasal and bronchial epithelial cells, as well as in mice after administration of HDM. Consistent with previously published data, we also demonstrated that chronic ER stress-mediated activation of ATF6 $\alpha$ led to increases in specific protein folding enzymes and subsequent activation of apoptotic executioner, caspase-3 [66, 77, 78]. Interestingly, knockdown of ATF6 $\alpha$ in human bronchial epithelial cells, as well as ATF6 $\alpha$ and ERP57 in mice, resulted in decreased airway epithelial apoptosis as measured by activation of caspase-3. As previously mentioned, It has been suggested that chronic activation of ATF6 $\alpha$ can lead to the induction of $\mathrm{CHOP}$ and subsequent up-regulation of proapototic Bak-mediated activation of caspases and apoptosis [49-52]. Accordingly, our results suggest that ATF6 $\alpha$ activation led to up regulation of CHOP as well as ERp57. SiRNA-mediated knockdown of ATF6a and ERp57 during HDM administration in mice resulted in decreased tissue resistance $(\mathrm{G})$ and tissue stiffness $(\mathrm{H})$ in mice, demonstrating an association between ER stress, apoptosis, and airway hyperresponsiveness to methacholine [67]. Collectively, these studies highlight mechanisms contributing to asthma pathogenesis, and that inhibition of ER stress responses may provide a potential therapeutic avenue to alleviate primary facets of allergic asthma.

Understanding how genetic factors modify UPR is of great relevance in chronic inflammatory diseases such as asthma. Moffatt et al. systematically mapped the effects of single nucleotide polymorphisms (SNPs) on the presence of childhood onset asthma by genome-wide association and found that genetic variants regulating Orosomucoid like 3 (ORMDL3), a gene encoding for the ERresident transmembrane protein ORMDL3, highly expressed in cells participating in the immune response are determinants of susceptibility to childhood asthma $[79,80]$. The ER is essential for the generation of $\mathrm{Ca}^{2+}$ signal transduction $[81,82]$ and as previously mentioned, alterations of protein folding or $\mathrm{Ca}^{2+}$ levels within the ER can result in the UPR. Cantero-Recasens et al. demonstrated that increased expression of ORMDL3 increased cytosolic $\mathrm{Ca}^{2+}$ levels, simultaneously reducing ER-mediated $\mathrm{Ca}^{2+}$ signaling and bolstering activation of UPR signaling molecules and target genes. siRNA-mediated knockdown of endogenous ORMDL3 reverted these effects, potentiating $\mathrm{ER} \mathrm{Ca}^{2+}$ release and attenuating the UPR [83], thus identifying a novel target in $\mathrm{ER} \mathrm{Ca}^{2+}$ signaling during disease pathogenesis.

While the association between ER stress, the UPR and asthma pathogenesis has yet to be clearly defined, accumulating evidence suggests that regulation of ER stress is a prospective molecular therapeutic target for inflammatory disorders. Chemical chaperones are small molecules that accumulate in the ER and directly facilitate protein folding, thereby inhibiting ER stress and associated 
UPR $[84,85]$. Interestingly, ER stress markers GRP78 and CHOP as well as ATF6 $\alpha$ signaling was dramatically reduced by treatment with 4-PBA in a model of steroid resistant asthma [71]. Furthermore, 4-PBA inhibited ER stress and substantially attenuated features of established allergic airway inflammation such as mucus metaplasia and collagen deposition, at doses compared to those previously reported for acute lung injury [86, 87]. Similarly, trimethylamine-N-oxide (TMAO) effectively inhibited CHOP and IL-13 production in a dose-dependent manner in a model of OVA-induced allergic airways disease. Despite success in mice, future studies will need to address the many open questions about the molecular mechanisms explaining these effects and the physiological significance of the various ER-stress signaling pathways in mediating inflammatory responses.

\subsection{Nuclear Factor-kappa B (NF-KB) signaling}

NF-KB is critically involved in many aspects of the immune system: from the development of organs and cells, to maintaining proper functioning of both the innate and adaptive immune systems. NF-kB is also a critical regulator of cell survival, cell death, and inflammation [88], and thus has been widely implicated in the etiology of diseases from bacterial infections to cancers [89]. Originally identified as a nuclear trans-acting factor that bound the $\mathrm{kB}$ light-chain enhancer in B cells, NF-kB was later found to be a family of five structurally related homo- and heterodimerizing transcription factors defined by a Rel homology domain [88]. Evolutionarily conserved from sponges to fruit flies to mammals, three of the NF-KB proteins, RelA (p65), RelB, and c-Rel are active following translation, whereas p50 and p52 are posttranslationally generated from the partial C-terminal degradation of precursors known as p105 and p100

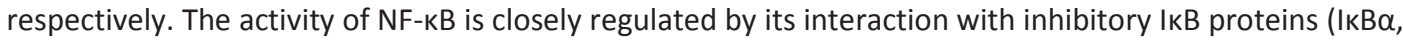
$\mathrm{I} \mathrm{KB} \beta$, and $(\mathrm{K} B \gamma)$ that have varying affinities for individual NF-KB dimers. Thus, in most cells, NF- $\mathrm{KB}$ is present as an inactive, IKB-bound complex in the cytoplasm.

The fundamental model posits the existence of at least two receptor-activated pathways termed the canonical and non-canonical NF-KB pathways. After receptor binding, IKB molecules are degraded, thus freeing NF-KB dimers to translocate into the nucleus and engage in transactivation of genes possessing consensus NF-KB binding sites [88]. Canonical NF-KB signaling is activated by various receptors, including microbial pattern recognition receptors such as TLRs, cytokine, and lymphocyte antigen receptors [88]. Signaling from these receptors ultimately converges on the IKK complex, consisting of the catalytic kinase subunits IKK $\beta$ and IKK $\alpha$ and a scaffolding protein called NF-KB essential modulator (NEMO or IKKY). Upon receptor ligation, stimulation of the IKK complex leads to phosphorylation of IKB $\alpha$ proteins by IKK $\beta$. This phosphorylation event triggers IKB ubiquitination and 
proteasomal degradation via the $26 \mathrm{~S}$ proteasome, releasing the sequestered NF-KB dimers RelA and p50. RelA and p50 translocate into the nucleus, bind DNA, and initiate transcription of proinflammatory and pro-survival genes [90].

The non-canonical (or alternative) pathway is largely for activation of p100/RelB complexes during B- and T-cell organ development. Receptor binding of specific agonists (e.g., Lymphotoxin B, B-cell activating factor, and CD40) leads to activation of the NF-KB inducing kinease NIK, which phosphorylates and activates the IKK $\alpha$ complex, which in turn phosphorylates the IKB domain of p100, leading to its partial proteolysis and liberation of the p52/RelB complex [90].

The association between NF-kB activation and inflammation has been demonstrated in various human diseases and animal models of disease, and NF-KB has been critically implicated in mediating inflammation using genetic approaches or chemical inhibitors. In the majority of cases, either epithelial cells of the infected tissue or tissue-resident hematopoietic cells, such as mast cells or dendritic cells (DCs), initiate the inflammatory response by triggering pro-inflammatory pathways through NF-KB in response to inflammatory stimuli [91]. Within the lung, NF-KB activation has correlated with the pathogenesis of chronic infections, lung cancer, and asthma [92-94]. Evidence for the role for NF-KB in airway inflammation has increased in both human patients with asthma and numerous models of allergic airways disease, with distinct manifestations of NF-KB activity within the bronchiolar epithelium [95]. Investigations involving mice that lack specific NF-KB subunits have demonstrated an abrogated response to antigenic and allergenic stimuli, thus revealing potential therapeutic targets aimed at inhibiting the biological activity of NF-kB.

It is well known that asthma, and other chronic inflammatory lung diseases, are associated with enhanced oxidative stress. NF-KB, as a redox sensitive transcription factor, has been demonstrated to require reactive oxygen species (ROS) for activation by proinflammatory stimuli [95]. In contrast, work from our laboratory has also demonstrated the ability of oxidants to inhibit NF-KB signaling within the lung epithelium via S-nitrosylation or S-glutathionylation of cysteine 179 of the IKK $\beta$ subunit [96]. Thus, it is likely that regulatory oxidative events could play a significant anti-inflammatory role in the lung by limiting the activation of NF-KB. 


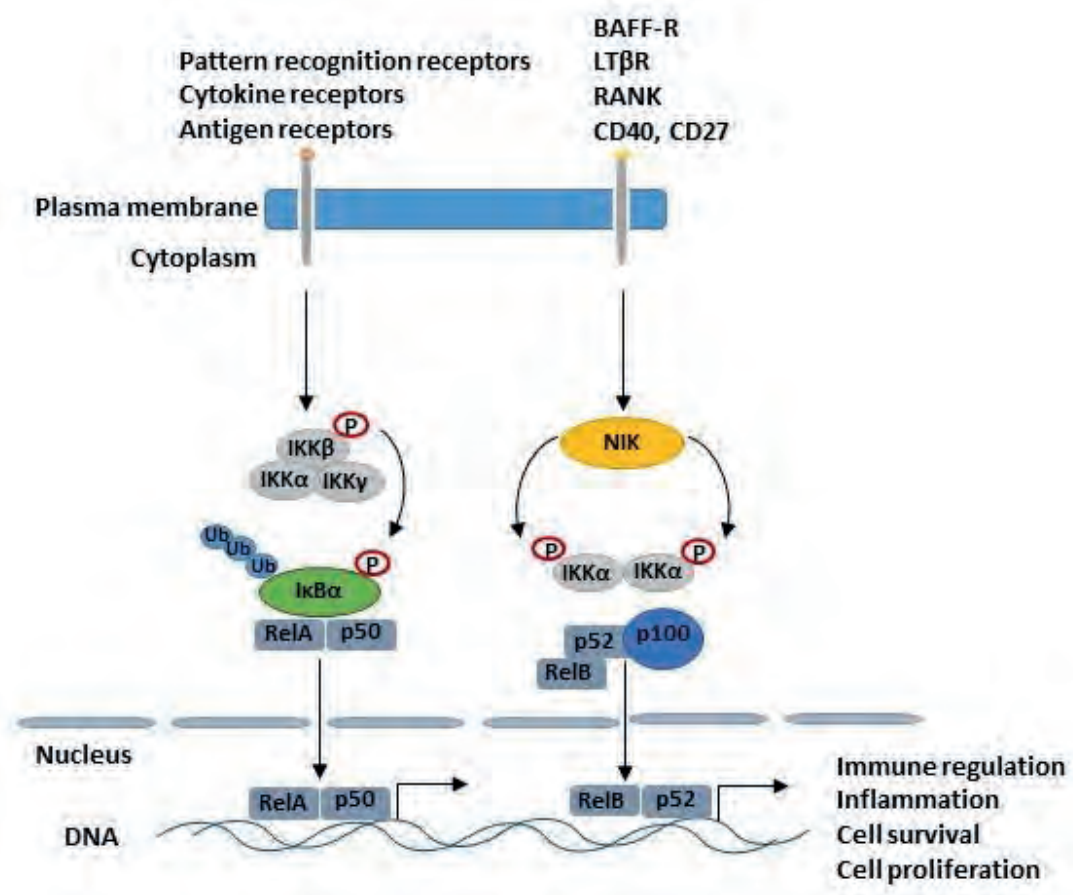

Figure 2. Canonical and non-canonical NF-KB activation. In the canonical pathway, NF-kB dimers p50/RelA are maintained in the cytoplasm by interaction with IkB $\alpha$. Upon stimulation, IKK is activated and phosphorylates IkB $\alpha$ leading to its ubiquitination and degredation by the $26 \mathrm{~S}$ proteasome. NF-kB subunits p50/RelA translocate into the nucleus and begin transcription of genes important in inflammation and immune regulation. Receptor binding in the non-canonical pathway leads to activation of NF-kB inducing kinase NIK, and phosphorylation and activation of the IKKa complex. Phosphorylation of 2 serine residues adjacent to the ankryn repeat C-terminal IkB domain of p100 results in partial proteolysis and liberation of the p52/RelB complex.

\subsection{Protein thiol redox homeostasis}

Oxidants, such as hydrogen peroxide, are largely thought to be harmful, as excessive amounts can induce cellular damage and have been implicated in a variety of diseases. Contrasting this notion, other studies have demonstrated that oxidants play a much more complex and not always harmful role in the regulation of cellular processes. A key manifestation of this is the oxidation and modification of cysteines (Figure 1), which holds significant biological importance as many of these modifications can control protein function [97-101]. While all cysteines contain a sulfhydryl moiety, not all are susceptible to oxidation. Reactive cysteines generally have a $\mathrm{pK}_{\mathrm{a}}$ value at or lower than neutral $\mathrm{pH}$, compared to the standard cysteine $\mathrm{pK}_{\mathrm{a}}$ around 8-8.5. This decrease in $\mathrm{pK}_{\mathrm{a}}$ is due in large part to the microenvironment surrounding the cysteine, which allows the formation of a thiolate anion (-S) that can be readily oxidized. Upon exposure to oxidants such as hydrogen peroxide, nitric oxide, peroxynitrite or other 
organic peroxides, the thiolate form of reactive cysteines can become oxidized to sulfenic acid (S-OH, sulfenylation), which is considered the "gateway oxidation" that can give rise to further oxidations [102]. Nitric oxide gives rise to nitrosylated protein cysteines (S-NO, S-nitrosylation), a post-translational modification thought to significantly contribute to biological function of nitric oxide [103]. Further reversible modifications of sulfenic acid include thiolation, cysteinylation, and S-glutathionylation. Sglutathionylation (P-SSG) represents the conjugation of the tri-peptide, glutathione (GSH), with a reactive cysteine in a protein. Both S-NO and S-SG are believed to be important in protection against irreversible protein oxidation, including the formation of cysteine sulfinic acid $\left(\mathrm{S}-\mathrm{O}_{2} \mathrm{H}\right)$ and sulfonic acid $\left(\mathrm{S}-\mathrm{O}_{3} \mathrm{H}\right)$, the hyper-oxidized forms of cysteine which typically lead to degradation. The exact mechanisms that govern inter-conversations between the various oxidized states in intact cells and tissues remain unknown. In addition to protecting the target protein from further oxidation, S-glutathionylation can have profound effects on protein activity, and protein activation and inactivation and other functional changes have been reported, depending on the target protein. Conversely, prolonged exposure of sulfenic acid intermediates to oxidants can generate irreversiblly oxidized sulfinic and sulfonic acid forms of cysteine.

The existence of enzymes involved in maintaining protein cysteine redox homeostasis provides further evidence for the biological importance of these modifications. Glutaredoxins (Grx), under normal physiological conditions, can specifically reverse S-glutathionylation of target proteins [96]. Thioredoxins (Trx), can reduce disulfide bonds as well as reverse S-nitrosylation of proteins [104], and sulfiredoxins (Srx) can reverse S-glutathionylation of certain targets $[105,106]$. Other enzymes involved in cysteine chemistry include protein disulfide isomerases (PDI), which are largely responsible for disulfide bond formation, as well as glutathione S-transferases (GST). Of these, GSTP was recently described as a putative catalyst of S-glutathionylation [107], although the specific targets of GSTP-catalyzed Sglutathionylation remain unknown.

\subsection{Redox perturbations in chronic inflammation and allergic asthma: ROS sources}

Despite the aforementioned importance of cysteine redox modifications, the source(s) of oxidants that regulate these events is difficult to determine. As has been previously discussed, numerous potential exogenous and endogenous sources of oxidants exist. Activation of NADPH oxidase in phagocytic cells, a quintessential factor in the host defense system, promotes intra-phagosomal ROS production to kill ingested pathogens. NADPH oxidase also contributes to activation of secreted proteolytic enzymes and receptor-mediated activation of pro-inflammatory signaling pathways [108]. While conflicting results 
exist, the role of active NADPH oxidase in allergic airway disease has been demonstrated by studies utilizing Gp91 ${ }^{\text {phox }}$ (subunit of flavocytochrome b558, the catalytic core of NADPH oxidase deficient mice $\left(\mathrm{Gp} 91^{\text {phox } / /}\right)$ in the Ovalbumin (OVA) model of allergic airway disease. It has been reported that Gp91 deficiency decreased ROS production, airway inflammation, and airway mucus production in Gp91 ${ }^{\text {phox- }}$ mice [109]. In contrast, it was also demonstrated that mice lacking $\mathrm{Gp} 91^{\text {phox }}$ exhibited enhanced influx of inflammatory cells and $\mathrm{T}_{H} 2$ cytokine levels $[110,111]$. Mechanistically, bone marrow-derived DCs from Gp91 $1^{\text {phox- } /-}$ animals were found to produce more IL-12 in response to LPS, suggesting enhanced $\mathrm{T}_{H} 1$ differentiation. Moreover, splenocytes isolated from sensitized Gp91 ${ }^{\text {phox- }- \text { mice }}$ produced less IL-13 in response to OVA challenge in vitro, indicating decreased $\mathrm{T}_{\mathrm{H}} 2$ differentiation. $\mathrm{T}$ cell differentiation appeared unaffected, however, as cytokine production by $\mathrm{T}$ cells was unaltered by the absence of Gp91 ${ }^{\text {phox }}$ under $T_{H} 0, T_{H} 1, T_{H} 2$ as well as $T_{H} 17$ polarizing conditions [109].

In DCs, Gp91 ${ }^{\text {phox }}$ is recruited to early phagosomes, mediating sustained production of low levels of ROS which cause alkalinization of the phagosomal lumen. Although, under certain conditions, such as prolonged contact between DCs and pro-inflammatory ligands, substantial production of ROS may occur and affect DC responses. Interaction of DCs with TLR ligands can for instance enhance the level of ROS production by DCs [112]. Phagosomal alkalinization normally observed in DCs however, was lost in bone

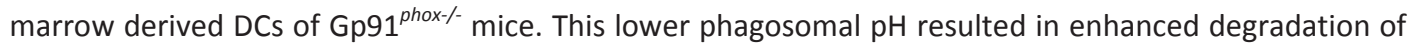
proteins and decreased efficiency of antigen cross-presentation [113, 114]. These studies implicate Gp91 ${ }^{\text {phox }}$ in the processing of antigens rather than in killing pathogens in DCs.

Gp91 ${ }^{\text {phox }}$ is also present in non-hematopoietic cells and its role in these cells in allergic airway disease has been investigated using chimeric mice. These experiments showed that the absence $G$ p $91^{\text {phox }}$ in nonhematopoietic cells resulted in reduced AHR in response to OVA, and inhibition of eosinophil migration across the endothelium. The effects on AHR could be bypassed by intratracheal administration of eosinophils, suggesting that the induction of NADPH oxidase in endothelium by VCAM1 is necessary for eosinophil recruitment [115].

Virtually all cell types express a non-phagocytic isoform of NADPH oxidase (NOX). Superoxide or hydrogen peroxide production by these NOX enzymes is tightly controlled and localized, and is involved in many physiological processes, relaying signals via posttranslational modifications in target proteins, notably via cysteine oxidations, described above. Their role in allergic airway inflammation and asthma has recently been elegantly reviewed [116, 117], and recent studies have also demonstrated their importance in the development of allergic airway disease. Duox1 is already known to be induced by the typical $T_{H} 2$ cytokines IL-4 and IL-13, and is associated with features of asthma such as mucin production. 
Interestingly, histamine, via the $\mathrm{H} 1$ receptor, has been shown to induce ROS production through Duox1 in primary human bronchial epithelial cells. Moreover, a synergistic effect with IL-4 inducing Duox1 gene and protein expression, and histamine further enhancing Duox activity has been observed [118]. Duox2 has also been shown to associate with TLR4 in bronchial and TLR2 in nasal epithelium upon HDM extract stimulation and shown to be necessary for the development of allergic airway disease and allergic rhinitis respectively [15]. The relevance of Duox-mediated ROS production in the development of allergic airway disease in vivo was furthermore shown using DUOXA deficient mice in the OVA model. Mice deficient in both Duox1 and 2, displayed reduced mucus cell metaplasia, lower levels of $\mathrm{T}_{\mathrm{H}} 2$ cytokines and somewhat surprisingly lower numbers of neutrophils, but not eosinophils in bronchoalveolar lavage fluid (BALF) in conjunction with attenuated AHR to methacholine [16]. To date, no reports on Duox levels in patients with asthma have been published, but these enzymes likely contribute to asthma pathology as they can affect the aforementioned processes.

Another non-phagocytic NAPDH oxidase of interest in asthma is NOX4 [17], as intrinsic NOX4 mRNA and protein expression in primary airway smooth muscle cells isolated from asthma patients was increased relative to healthy controls. Importantly, the enhanced contractility displayed by asthmatic smooth muscle cells was attenuated following NOX4 knockdown and pharmacological inhibition [17]. Additionally, NOX4 is required for expression of differentiation markers in smooth muscle cells in vitro and mediates proliferation and hypertrophy induced by TGF- $\beta$ [18]. NOX4 also has been implicated in other disorders of the lung, such as IPF [119], and initiation of NOX4-induced ROS production at the surface of fibroblasts can enhance epithelial cell apoptosis [19]. Together, these NOX-mediated cellular responses are thought to be important in the pathophysiology of asthma, and application of the general NOX inhibitor apocynin in the OVA model decreased AHR and inflammation and affected activation of redox sensitive transcription factors including activator protein-1 (AP1) and Nuclear Factor kappa B (NF$\mathrm{KB}$ ) in vitro [20]. Moreover, a prior study observed attenuation of AHR by apocynin treatment, but did not demonstrate effects on inflammation [21]. Importantly, a number of allergens have been shown to contain NADPH oxidase activity as well [22]. A variety of pollen grain extracts not only contained intrinsic NADPH oxidase activity, but this activity was crucial to the induction of ROS production in mouse lung epithelial cells, as well as markers of oxidative stress prior to inflammatory cell influx [26]. Furthermore, this initial induction of local oxidative stress by these extracts was important in augmenting antigeninduced allergic airway inflammation. It has also been shown that challenging mice with ragweed extract lacking NADPH oxidase activity resulted in diminished recruitment of eosinophils and mucus metaplasia, an effect that is not accompanied by altered production of $\mathrm{T}_{\mathrm{H}} 2$ cytokines [22]. 
Lastly, exacerbations of allergic airway disease are often associated with enhanced exposure to oxidants and oxidizing particulates and gases such as pollution and cigarette smoke. Air pollution is thought to contribute to the increase in incidence of allergy and allergic asthma in industrial countries. One reason for this increase could be the interaction of pollutants with known allergens thereby increasing their allergenicity, or with non-allergic substances causing them to become allergens. These possibilities were investigated in vitro by examining the effect of ozone fumigation of ragweed pollen. Ozone fumigation was found to cause damage to the pollen membrane system, which can lead to enhanced release of pollen-associated lipid immune-modulators [27], but it did not affect the content of allergens per se. Ozone fumigation did significantly increase NAD(P)H oxidase enzyme activity [28] however, which could increase the pollen's allergenicity [26].

\subsection{Cysteine redox perturbations in chronic inflammation and allergic asthma: Glutathione and its regulatory enzymes}

Within the lung tissue, thiols in the form of glutathione (GSH) and sulfhydryl groups of proteins are among the most susceptible oxidant-sensitive targets and can be either reversibly oxidized to sulfenic acids $(-\mathrm{SOH})$ or disulfides $(\mathrm{S}-\mathrm{S})$, or irreversibly oxidized to sulfinic $\left(-\mathrm{SO}_{2} \mathrm{H}\right)$ and sulfonic $\left(-\mathrm{SO}_{3} \mathrm{H}\right)$ acids, as discussed earlier. Although reversible oxidations are believed to protect proteins from irreversible oxidation, the sulfenic acid moiety is very unstable and readily reacts with other thiols to form intra- or intermolecular disulfides, potentially altering protein function. Protein S-glutathionylation (PSSG), the reversible conjugation of a GSH molecule to a reactive cysteine via a disulfide bond, is a critical regulator of cellular function by controlling diverse signaling pathways and has been associated with inflammation. Accumulation of PSSG occurs in different cell types under a variety of oxidative conditions [120]. Although total levels of glutathione in BALF, sputum and EBC have been reported to be increased in asthma, few studies examined levels of GSSG and these show conflicting data as reviewed by Reynaert, N.L. [121]. A recent study on a relatively large cohort of children predominantly with severe asthma found increased levels of GSSG in both macrophages the BALF, which were associated with decreased HDAC activity, increased apoptosis and impaired phagocytosis [122]. An associated study also found lower levels of reduced GSH and protein cysteines in the airways and plasma of children with severe asthma. These results were associated with dysfunctional Nrf2 activity, despite elevated Nrf2 mRNA and protein expression [123]. Regardless, evidence suggests that the state of GSH redox equilibrium in immune cells is crucial in determining the balance of $T_{H} 1 / T_{H} 2$ immune responses and antigen presentation. For instance, lowering intracellular GSH levels was shown to negatively impact antigen 
processing in DCs, especially of antigens containing disulfide bonds [2]. Nrf2 has also been shown to influence antigen presentation activity by controlling the redox equilibrium of DCs [124]. These data correlate with the reports described above on the role of $\mathrm{Gp} 91^{\text {phox }}$ in antigen processing in DCs. Further, higher intracellular levels of reduced GSH favored $\mathrm{T}_{\mathrm{H}} 1$ development through IL-12 production in antigen presenting cells [23]. Conversely, exposure of macrophages to the $T_{H} 1$ cytokine IFN $\gamma$ increased the GSH/GSSG ratio, whereas exposure to the $T_{H} 2$ cytokine IL-4 decreased this ratio [24]. Administration of $\gamma$ glutamylcysteinylethyl ester, a GSH precursor, to monocytes in vitro as well as in the OVA model in vivo also increased IL-12 and suppressed production of $\mathrm{T}_{\mathrm{H}} 2$ cytokines IL- 4 and IL- 5 and eosinophil chemotaxis [25].

\section{Glutathione peroxidase}

At present, it is not clear if the expression of the enzymes involved in GSH synthesis is altered in asthmatics. However, enzymes that regulate GSH redox status and that use GSH have been studied in the context of asthma. Glutathione peroxidase 3 (GPx3) or extracellular GPx, the most abundant GPx isoform in the lung, was found to be increased in the BALF and bronchial epithelial cells of asthma patients compared to healthy controls [125]. GPx1, the most abundant intracellular GPx isoform, was increased in the OVA model of allergic asthma, and furthermore, GPx1-deficient mice exhibited attenuated eosinophilia, goblet cell hyperplasia, collagen deposition and AHR. Moreover, in vitro studies demonstrated that CD4+ $T$ helper cells derived from GPx1-deficient mice exhibited enhanced proliferation and produced more ROS and IL-2 after stimulation compared to WT cells. Gpx1 $1^{-/}$cells were also predominantly $T_{H} 1$ and $T_{H} 17$-biased. Together, these data indicate that the effects of GPx1 on immune cell proliferation and differentiation may predominate over its effects on protection against oxidative damage [126]. Expression of GPx2 was shown to be elevated in the airway epithelium in the OVA model of allergic airway disease, but unlike GPx1-deficient mice, GPx2-deficient mice showed enhanced airway inflammation and reactivity in this model [127]. Discrete localization and functions of different GPx isoforms could explain both protective versus enhancing roles in allergic airway disease. With respect to the latter, indications exist that GPx2 might function less as a GSH peroxidase and could be more involved in inhibiting prostaglandin synthesis and enhance other anti-inflammatory mechanisms [127].

Glutathione-S-transferase

Members of the glutathione-S-transferase (GST) superfamily have been under investigation in asthma since they are critical in the protection of cells against toxic products of ROS-mediated reactions. GSTP1 is the predominant cytosolic GST in the human lung, and is highly expressed in bronchial type 2 
pneumocytes. GSTP1 is located on chromosome 11q13, a locus linked to asthma susceptibility. Furthermore, specific GSTP1 polymorphisms are strongly associated with asthma [128]. Interestingly, challenge of ragweed-sensitive patients with allergen alone or in combination with diesel exhaust particles induced IgE and histamine production in patients expressing lle105, a variant of GSTP1 [129]. GSTP expression was found to be decreased in nasal epithelial cells derived from children with asthma compared to non-asthmatic children. Further, GSTP1 is functionally involved in specific pathophysiological processes that underlie clinical features of asthma. Eosinophilia, goblet cell hyperplasia, IL-5 levels, airway remodeling and lung resistance were enhanced in GSTP-deficient mice compared to WT controls in the OVA model. As in humans, strain-dependent effects were observed in mice that correlated with endogenous levels of GSTP expression [130]. GSTP-null mice also displayed increased cysteine disulfide levels in response to HDM relative to WT animals [116].

\section{Glutaredoxin}

Glutaredoxin (Grx) is a specific and well-characterized catalyst of protein deglutathionylation [120]. A member of the thiol-disulfide oxidoreductase family, Grx promotes reversible reduction of protein-SSG to free sulfhydryl groups (Figure 1). In addition, Grx is unique in that it regenerates itself through the oxidation of GSH, notably at high concentrations of GSH [131]. In this reaction, Grx itself is Sglutathionylated at Cys-22, and the reduced state of Grx is restored by the coupling GSH to a second GSH molecule, forming a glutathione disulfide (GSSG) [96]. So far, Grx and PSSG have only been reported in one study in patients with asthma. Our group has recently shown that Grx1 levels are increased, and PSSG levels are decreased, in the sputum from asthmatics. These alterations in Grx1 seemed largely independent of the inflammatory cells present in the sputum, but expression of Grx1 was increased in bronchial epithelial cells isolated from asthma patients [132]. PSSG levels on the other hand were only decreased in asthmatics with a dominant eosinophilic or neutrophilic sputum cellular profile, and positively correlated with the percentage of lymphocytes in the sputum. Sputum PSSG levels in asthma are therefore likely influenced not only by Grx1 levels, but also by other factors such as oxidative changes related to inflammation. Whether decreases in sputum PSSG are associated with enhanced protein deglutathionylation and whether this increases the vulnerability of these proteins to oxidative damage has yet to be determined. Importantly, decreased lung function was associated with higher sputum Grx1 levels and lower PSSG levels. Additionally, PSSG levels correlated with asthmatic controls in neutrophilic patients [132]. These data correspond with the increases in Grx1 expression and activity predominantly in bronchial epithelium in the OVA model of allergic airway disease [96]. A follow-up 
study also demonstrated that additional OVA challenges lead to further increases in Grx1 expression within the whole lung. No clear effects on lung PSSG were observed in WT mice in response to OVA, but marked increases in PSSG content was detected 48h after repeated antigen challenge in the lungs of Grx1-deficient mice. To investigate the impact of Grx1/PSSG alterations on the development of allergic airway disease, Grx1-deficient mice $\left(G / r \times 1^{\%}\right)$ were sensitized and challenged with OVA. No effect of Grx1 ablation on OVA-specific IgE levels were observed, but inflammatory cell influx and expression of proinflammatory mediators were decreased in $\mathrm{Glr} \mathrm{1}^{-/}$mice. WT and $\mathrm{Glr} \times \mathrm{1}^{-1}$ mice demonstrated

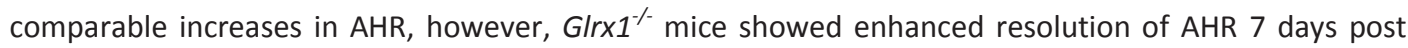
challenge cessation. This was accompanied by marked decreases in mucus metaplasia and IL-13 expression, in the absence of effects on inflammation. These results demonstrate that the Grx1/Sglutathionylation redox status in mice is a critical regulator of AHR and its resolution, and suggest that avenues to increase S-glutathionylation of specific targets may attenuate AHR [133].

\section{Thioredoxin}

Thioredoxin (Trx), represents another class of thiol-oxidoreductases important in cellular redox homeostasis, for which numerous target proteins have been identified, and has emerged as a critical regulator of biological processes [134]. Serum levels of Trx were significantly increased in patients during asthmatic exacerbation, and serum Trx levels correlated with lung function and eosinophil cationic protein levels during these times. Levels of serum Trx may therefore correspond to the extent of airway obstruction and eosinophil activation in the lung, and could represent a possible protective response [135].

This latter possibility alludes to several animal studies that show a beneficial effect of Trx in animal models of asthma. Reduced eosinophilia, mucus metaplasia and AHR were observed in Trxoverexpressing mice, for example, while effects on $T_{H} 2$ responses were not [136]. Furthermore, studies have shown that exogenously administered recombinant Trx suppressed allergic inflammation in animal models of asthma. Intraperitoneal administration of recombinant human (rh) Trx1 during antigen challenge significantly inhibited airway remodeling, lung eosinophilia and AHR, and resulted in decreased eotaxin, macrophage inflammatory protein-1alpha and IL-13 expression in the lung. Importantly, administration of rhTrx1 significantly ameliorated existing goblet cell hyperplasia in OVA-sensitized and challenged WT mice [137]. Administration of WT Trx also suppressed AHR and increased mRNA expression of several $\mathrm{T}_{H} 1$ cytokines including including IL-1 $\beta$ and IL-18 in the lungs of OVA-sensitized mice. However, the 32S/35S mutant Trx, the catalytically-inactive form of this enzyme, did not affect these endpoints, confirming that the effects of Trx are dependent on its enzymatic activity. Trx may also 
regulate the $T_{H} 1$ response by increasing expression of IFNY and IL-12 in monocytes and T cells in vitro, but these effects were not observed in the Trx transgenic mice described above [136]. The effects of Trx on AHR and airway inflammation are subject to some controversy however [138], and differences in genetic background, immunization protocols, as well as the potential divergent functions of intracellular vs extracellular and endogenous vs exogenous Trx could be responsible for these discrepancies. Repression of eosinophilia could be attributed to multiple mechanisms of action of Trx. Interestingly, preincubation with Trx was found to directly suppress eotaxin and RANTES-induced chemotaxis of eosinophils. Trx did not affect expression of the receptor of these two chemokines, CCR3, but attenuated the downstream activation of ERK 1/2 and p38 MAPK [139].

The anti-inflammatory effects of Trx are generally thought to be dependent on its catalytic function. Importantly however, Trx also directly attenuates eosinophil and neutrophil chemotaxis, and may therefore have clinical benefits. Along these lines, it is important to note that the half-life of Trx in lung tissue is 6 times longer than in sera (51.3 hrs vs 8.5 hrs) [140].

\subsection{Potential for therapeutic intervention and targeting of oxidative events and cysteine oxidations in asthma}

Asthma represents a major health concern worldwide and while current mainstay therapies, namely $\beta$ agonists and glucocorticoids reduce airway inflammation, reverse bronchoconstriction and improve quality of life, there remains a significant patient population for whom these treatments are ineffective [29]. Additionally, these treatments have little or no effect on structural alterations of the disease, and do not reverse or prevent disease progression. Current popular belief holds that oxidants are "bad," contributing to cellular damage and tissue pathology. The possibility that oxidants can exert regulatory biological functions, and even constitute anti-inflammatory mechanisms, however, is not implausible and has great clinical relevance. Given that our understanding of means to inhibit inflammation in allergic asthma is incomplete to date, ongoing and future studies are significant in that they attempt to identify the contribution of specific ROS-producing enzymes and resultant modification of protein cysteine residues. We envision the use of chemical compounds to specifically enhance $S$-glutathionylation in the context of allergic asthma. Of direct translational relevance NOV-002, a dimeric version of oxidized glutathione (GSSG), which may enhance PSSG by altering the GSH:GSSG ratio [122, 123]. Such studies have the potential to unravel beneficial redox reactions catalyzed by oxidants that are targeted with the goal to dampen the extent of and enhance resolution of allergic inflammation. 


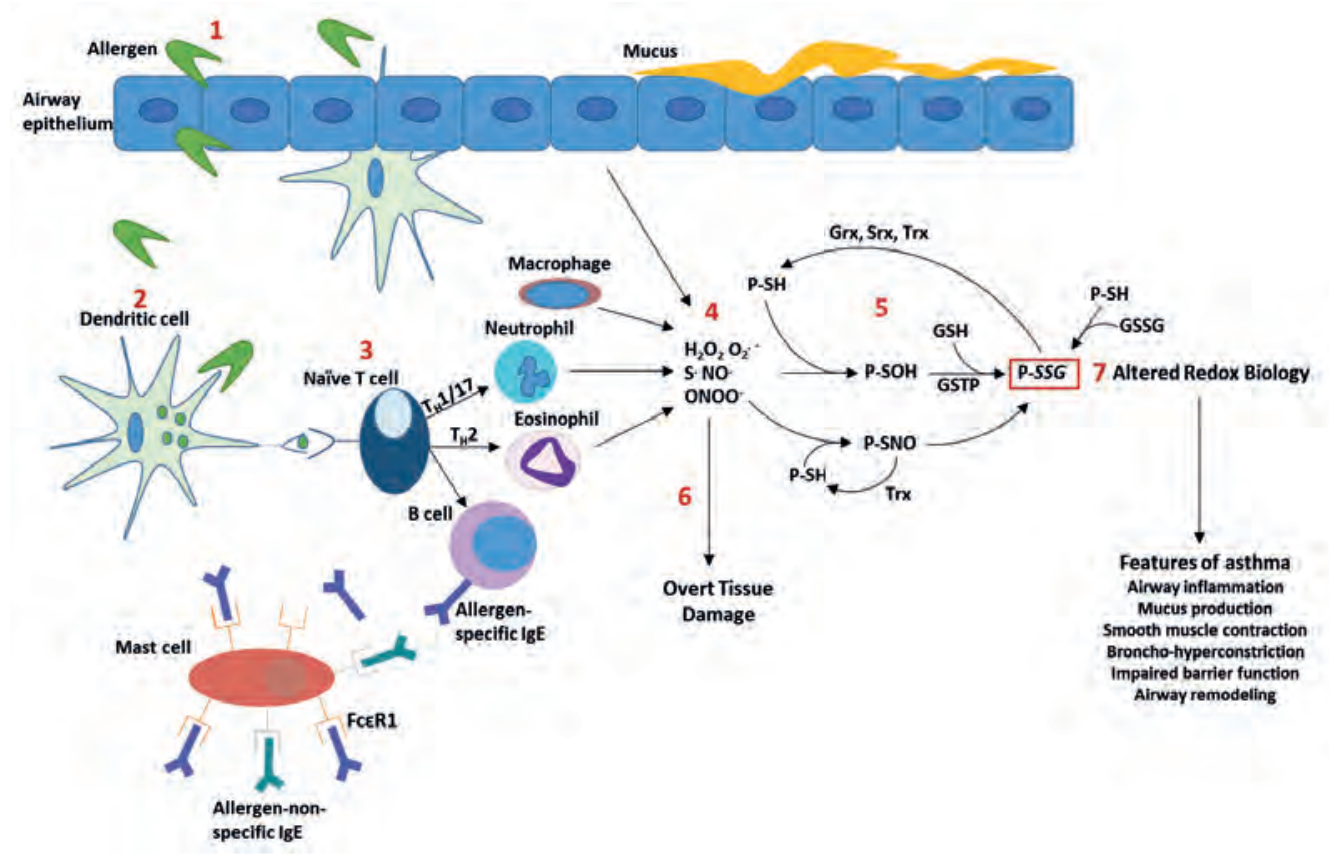

Figure 3. Overview of allergen-initiated innate and adaptive immune responses and associated changes in protein thiol redox status. (1) Inhalation of allergens leads to disruption of epithelial cell homeostasis and impaired barrier function, allowing the allergen to interact with dendritic cells and other immune cells, including T cells. (2) Allergens are engulfed and processed by dendritic cells, which present antigenic epitopes to naïve T cells. (3) Naïve T cells polarize leading to recruitment of neutrophils and eosinophils (innate immune response) and activation of B cells, driving the adaptive immune response. (4) Reactive oxygen and nitrogen species are produced by inflammatory cells and structural cells including epithelial cells, resulting in oxidative modifications of reactive cysteines within proteins (P) (5). The downstream effects of oxidant overproduction have traditionally been associated with tissue damage (6). However, altered protein thiol redox biology (7) has emerged as a putative mechanism where oxidants contribute to the chronic features of asthma. This (patho) biological role of oxidants has the potential to be exploited towards the development of new and targeted therapeutics for patients with asthma. $\mathrm{H}_{2} \mathrm{O}_{2}$, hydrogen peroxide; $\mathrm{O}_{2}{ }^{-}$, superoxide; $\mathrm{S}$, thiyl; NO nitric oxide; ONOO peroxynitrite; $\mathrm{P}-\mathrm{SH}$, protein thiol; $\mathrm{P}-\mathrm{SOH}, \mathrm{protein}$ sulfenic acid; P-SNO, S-nitrosylation; P-SSG, S-glutathionylation; Grx, glutaredoxin; Srx, sulfiredoxin; Trx, thioredoxin; GSTP, glutathione S-transferase pi; GSSG, glutathione disulfide.

\subsection{Mouse models of asthma}

To better understand the mechanisms underlying the asthmatic response, studies have characterized the physiological and inflammatory responses in genetically diverse inbred mouse strains following allergen sensitization and exposure. From these studies, it has been shown that the strains differ in their ability to mount an allergen-induced asthmatic response. Whitehead et al. described C57BI/6 mice to behave differently from Balb/C mice in models of allergic inflammation [6]. The C57BI/6 strain has been 
demonstrated to have a predominantly $T_{H} 1$ immune response to antigen, whereas a predominantly $T_{H} 2$ response is elicited in Balb/C mice [6]. Sensitization and challenge with Ovalbumin (OVA) has shown to induce elevated levels of IgE and more pronounced AHR in Balb/C than C57BI/6 mice [141, 142]. Alternatively, $\mathrm{C} 57 \mathrm{BI} / 6$ mice accumulated more eosinophils dispersed throughout the lung as compared to Balb/C mice, which exhibited eosinophilic infiltration to a lesser extent and were primarily localized around the airways $[6,142]$. Alcorn et al. further investigated these disparate inflammatory cell profiles and the functional importance of NF-KB activation within the airway epithelium in Balb/C and C57BI/6 mice, to unravel whether the extent or locale of NF-kB could explain the differences in intensity of allergic airway disease between these strains [143]. OVA-sensitized mice displayed prominent increases in nuclear RelA translocation in the bronchiolar epithelium however, no nuclear localization was apparent in $\mathrm{C} 57 \mathrm{BI} / 6$ mice subjected to the same protocol. Interestingly, assessment of NF-KB DNA binding in the whole lung by EMSA demonstrated that NF-KB binding increased in response to OVA to a similar extent in both strains of mice [143]. This suggests that C57BI/6 mice are not refractory to NF-kB activation compared with Balb/C mice in response to antigen, but rather it is the location of NF- $\mathrm{KB}$ activation that differs between the strains. Additionally, Balb/C mice exhibited significantly higher levels of TNF- $\alpha$ (a potent activator of the NF-kb pathway) in BAL fluid than C57BI/6 mice in response to antigen challenge. Furthermore, neutralization of TNF- $\alpha$ activity attenuated OVA-induced nuclear localization of RelA in the airway epithelia of Balb/C mice [143], strongly suggesting the involvement of TNF- $\alpha$ in the rapid activation of epithelial NF-KB in Balb/C mice. While it is not clear which of these two backgrounds best reflects human allergic asthma, experiments in both backgrounds have contributed significantly to our current understanding of the mechanisms of this disease.

Understanding the role of environmental factors in asthma has benefitted from mouse models, specifically the role of allergy and $\mathrm{T}_{\mathrm{H}} 2$ cell responses in mice with allergic asthma. Mice can be sensitized to a number of foreign proteins such as OVA, house dust mite or cockroach extracts, as well as Aspergillus fumigatus and ragweed pollen, by immunization with adjuvants such as aluminum hydroxide (alum) or low doses of endotoxin [29]. The most widely-used model of asthma involves allergic sensitization by intraperitoneal injections of OVA complexed with the $T_{H} 2$ adjuvant alum [144]. After sensitization, antigen-specific $\mathrm{T}_{\mathrm{H}} 2$ cells are induced and recruited into the lungs and cause the development of eosinophil-predominant inflammation and AHR in response to allergen challenge. Repeated inhaled administration of OVA into the lungs leads to common features of human allergic asthma, such as mucous secretion, goblet cell hyperplasia, and airway remodeling. However, the intraperitoneal sensitization process in mice might not reflect the natural process of sensitization that 
occurs in humans, differences that might reflect the impact of the unique mucosal environment of the lung on developing immune responses [144]. As previously mentioned, airway epithelial cells are the first line of defense against the inhaled environment, and are crucial in initiating activation of innate and adaptive immune responses. It has been demonstrated that LPS-mediated allergen sensitization through the airway followed by antigen challenge can provoke an IL-17 response, shown to be complemented by the induction of $T_{H} 2$ immunity [144]. Additional studies exposing mice to a combination of both flagellin, a TLR5 agonist, and OVA via the airways elicited an even more robust allergic phenotype [145] hence, the strength and nature of responses to antigen challenge are strongly influenced by the methods in which the mice are sensitized.

In addition to the issue of route of sensitization, several points of criticism have been raised questioning the physiological and clinical relevance of using OVA to model human asthma such as tolerance following extended exposure and perhaps most importantly, ovalbumin is not among the myriad of allergens considered central triggers for asthma [146]. The most commonly used allergen is house dust mite extract (HDM), a multifaceted allergen to which $50-85 \%$ of asthmatics are allergic [147]. HDM extract contains many allergenic components including fecal matter, LPS, chitin and proteins such as Dermatophagoides pteronyssinus, all of which are thought to contribute to the allergic response. Exposure to HDM induces allergic airways disease in mice through TLR4 activation within airway structural cells, and production of pro-inflammatory cytokines thymic stromal lymphopoietin (TSLP), granulocyte-macrophage colony stimulating factor (GM-CSF), interleukin 25 (IL-25), and IL-33 [148].

Finally, transgenic approaches such as overexpression of the $\mathrm{T}_{\mathrm{H}} 2$-specific transcription factor GATA3 or the $T_{H} 17$ transcription factor RORyt have also provided an effective method to study the role of $T_{H} 2$ and $T_{H} 17$ adaptive immune responses following allergic sensitization in vivo [148, 149]. With the broadening of mouse models, investigators are able to examine the diverse underlying mechanisms resulting in the inflammatory phenotypes that resemble endotypes of clinical asthma, allowing for close comparison and identification of important pathophysiologic biomarkers.

\section{Aims and outline of the thesis}

Allergic asthma is a complex disease characterized by chronic airway inflammation and airway hyperreactivity (AHR); it is a serious public health issue, affecting approximately 300 million people worldwide [3]. Our current understanding of allergic asthma has been propelled by mouse models of allergic airways disease, illuminating a role for airway epithelial cells and changes in redox regulation in the organization of inflammatory responses. Further investigation into the functional significance of specific 
modules of redox signaling (notably S-glutathionylation and glutaredoxin) in ER stress and NF-KB activation has the potential to elucidate novel biochemical pathways that control the pathophysiology of allergic airways inflammation, and potentially lead to novel therapeutic strategies.

A major goal of this thesis was to examine the impact of regulation of protein thiol redox changes in the pathogenesis of allergic airways disease. In this thesis we focused on two different protein thiol oxidoreductases, ERp57, and Glrx1. ERp57 controls protein disulfides and has been involved in ER stress while Glrx1 has been involved in protein S-glutathionylation and pro-inflammatory signaling, notably the regulation of NF-KB. The chapters that comprise this thesis aim to describe the molecular mechanisms of ER stress and NF-KB activation during HDM-induced allergic airways disease, as well as the role of glutaredoxin-1 (Grx1) in the pathogenesis of allergic inflammation through the use of G/rx $1^{-1}$ mice in the ovalbumin (OVA) and HDM models of allergic inflammation. Chapter 2 examines the contribution of ER stress transducer activating transcription factor 6 and protein disulfide isomeraseERp57 (a protein thiol oxidoreductase) in the pathogenesis of primary facets of allergic asthma, using specific small interfering RNAs (siRNAs) in vivo. Chapter 3 describes a comprehensive investigation of the kinetics of the activation of NF-KB and the ensuing allergic response to HDM, from $1 \mathrm{~h}$ after a single challenge up to $72 \mathrm{~h}$ following 15 instillations. Additionally, this chapter illustrates the functional importance of epithelial NF-KB in response to HDM through the use of a $\mathrm{CC} 10-\mathrm{IKB} \alpha_{S R}$ mouse refractory to IKB $\alpha$ degradation and subsequent NF-KB activation in the lung epithelium. Chapter 4 details the characterization of the genetic ablation of the protein thiol oxidoreductase GIrx1 in the acute and resolution phases of OVA-induced allergic inflammation. Chapter 5 expands upon the results in Chapter 4, and describes the pathophysiological role of Grx1 following chronic exposure to HDM extract.

Finally, a summarization of the overall findings and discussion, as well as future directions will be presented in Chapter 6. 


\section{References}

1. Hansbro, P.M., G.E. Kaiko, and P.S. Foster, Cytokine/anti-cytokine therapy - novel treatments for asthma? Br J Pharmacol, 2011. 163(1): p. 81-95.

2. Short, S., et al., Defective antigen processing correlates with a low level of intracellular glutathione. Eur J Immunol, 1996. 26(12): p. 3015-20.

3. Kim, H.Y., R.H. DeKruyff, and D.T. Umetsu, The many paths to asthma: phenotype shaped by innate and adaptive immunity. Nat Immunol, 2010. 11(7): p. 577-84.

4. Narayanankutty, A., et al., Biochemical pathogenesis of aspirin exacerbated respiratory disease (AERD). Clin Biochem, 2013. 46(7-8): p. 566-78.

5. Holgate, S.T., A brief history of asthma and its mechanisms to modern concepts of disease pathogenesis. Allergy Asthma Immunol Res, 2010. 2(3): p. 165-71.

6. Whitehead, G.S., et al., Allergen-induced airway disease is mouse strain dependent. Am J Physiol Lung Cell Mol Physiol, 2003. 285(1): p. L32-42.

7. Steinman, R.M., The dendritic cell system and its role in immunogenicity. Annu Rev Immunol, 1991. 9: p. 271-96.

8. May, M., Drug companies hope to breathe life into asthma pipeline. Nat Med, 2011. 17(6): p. 642-3.

9. Hamelmann, E., et al., Role of IgE in the development of allergic airway inflammation and airway hyperresponsiveness--a murine model. Allergy, 1999. 54(4): p. 297-305.

10. McKeage, K., Omalizumab: a review of its use in patients with severe persistent allergic asthma. Drugs, 2013. 73(11): p. 1197-212.

11. Pavord, I.D., et al., Mepolizumab for severe eosinophilic asthma (DREAM): a multicentre, doubleblind, placebo-controlled trial. Lancet, 2012. 380(9842): p. 651-9.

12. Ortega, H.G., et al., Mepolizumab Treatment in Patients with Severe Eosinophilic Asthma. N Engl J Med, 2014. 371(13): p. 1198-1207.

13. Souwer, Y., et al., IL-17 and IL-22 in atopic allergic disease. Curr Opin Immunol, 2010. 22(6): p. 821-6.

14. Hamid, Q. and M. Tulic, Immunobiology of asthma. Annu Rev Physiol, 2009. 71: p. 489-507.

15. Ryu, J.H., et al., Distinct TLR-mediated pathways regulate house dust mite-induced allergic disease in the upper and lower airways. J Allergy Clin Immunol, 2013. 131(2): p. 549-61.

16. Chang, S., et al., Dual oxidase regulates neutrophil recruitment in allergic airways. Free Radic Biol Med, 2013. 65: p. 38-46.

17. Sutcliffe, A., et al., Increased nicotinamide adenine dinucleotide phosphate oxidase 4 expression mediates intrinsic airway smooth muscle hypercontractility in asthma. Am J Respir Crit Care Med, 2012. 185(3): p. 267-74.

18. Sturrock, A., et al., Transforming growth factor-beta1 induces Nox4 NAD(P)H oxidase and reactive oxygen species-dependent proliferation in human pulmonary artery smooth muscle cells. Am J Physiol Lung Cell Mol Physiol, 2006. 290(4): p. L661-L673.

19. Waghray, M., et al., Hydrogen peroxide is a diffusible paracrine signal for the induction of epithelial cell death by activated myofibroblasts. FASEB J, 2005. 19(7): p. 854-6.

20. Kim, S.Y., et al., Anti-inflammatory effects of apocynin, an inhibitor of NADPH oxidase, in airway inflammation. Immunol Cell Biol, 2012. 90(4): p. 441-8.

21. Muijsers, R.B., et al., Apocynin and $1400 \mathrm{~W}$ prevents airway hyperresponsiveness during allergic reactions in mice. Br J Pharmacol, 2001. 134(2): p. 434-40. 
22. Bacsi, A., et al., Effect of pollen-mediated oxidative stress on immediate hypersensitivity reactions and late-phase inflammation in allergic conjunctivitis. J Allergy Clin Immunol, 2005. 116(4): p. 836-43.

23. Murata, Y., M. Amao, and J. Hamuro, Sequential conversion of the redox status of macrophages dictates the pathological progression of autoimmune diabetes. Eur J Immunol, 2003. 33(4): p. 1001-11.

24. Dobashi, K., et al., Regulation of LPS induced IL-12 production by IFN-gamma and IL-4 through intracellular glutathione status in human alveolar macrophages. Clin Exp Immunol, 2001. 124(2): p. 290-6.

25. Koike, Y., et al., Glutathione redox regulates airway hyperresponsiveness and airway inflammation in mice. Am J Respir Cell Mol Biol, 2007. 37(3): p. 322-9.

26. Boldogh, I., et al., ROS generated by pollen NADPH oxidase provide a signal that augments antigen-induced allergic airway inflammation. J Clin Invest, 2005. 115(8): p. 2169-79.

27. Traidl-Hoffmann, C., et al., Lipid mediators from pollen act as chemoattractants and activators of polymorphonuclear granulocytes. J Allergy Clin Immunol, 2002. 109(5): p. 831-8.

28. Pasqualini, S., et al., Ozone affects pollen viability and $N A D(P) H$ oxidase release from Ambrosia artemisiifolia pollen. Environ Pollut, 2011. 159(10): p. 2823-30.

29. Umetsu, D.T., et al., Asthma: an epidemic of dysregulated immunity. Nat Immunol, 2002. 3(8): p. 715-20.

30. Swamy, M., et al., Epithelial decision makers: in search of the 'epimmunome'. Nat Immunol, 2010. 11(8): p. 656-65.

31. Wullaert, A., M.C. Bonnet, and M. Pasparakis, NF-kappaB in the regulation of epithelial homeostasis and inflammation. Cell Res, 2011. 21(1): p. 146-58.

32. Cheng, D.S., et al., Airway epithelium controls lung inflammation and injury through the NFkappa B pathway. J Immunol, 2007. 178(10): p. 6504-13.

33. Everhart, M.B., et al., Duration and intensity of NF-kappaB activity determine the severity of endotoxin-induced acute lung injury. J Immunol, 2006. 176(8): p. 4995-5005.

34. Walsh, E.R., K. Stokes, and A. August, The role of eosinophils in allergic airway inflammation. Discov Med, 2010. 9(47): p. 357-62.

35. Holgate, S.T., The airway epithelium is central to the pathogenesis of asthma. Allergol Int, 2008. 57(1): p. 1-10.

36. Comhair, S.A. and S.C. Erzurum, Redox control of asthma: molecular mechanisms and therapeutic opportunities. Antioxid Redox Signal, 2010. 12(1): p. 93-124.

37. Zhang, K. and R.J. Kaufman, From endoplasmic-reticulum stress to the inflammatory response. Nature, 2008. 454(7203): p. 455-62.

38. Kaufman, R.J., Stress signaling from the lumen of the endoplasmic reticulum: coordination of gene transcriptional and translational controls. Genes Dev, 1999. 13(10): p. 1211-33.

39. Townsend, D.M., S-glutathionylation: indicator of cell stress and regulator of the unfolded protein response. Mol Interv, 2007. 7(6): p. 313-24.

40. Shi, Y., et al., Identification and characterization of pancreatic eukaryotic initiation factor 2 alphasubunit kinase, PEK, involved in translational control. Mol Cell Biol, 1998. 18(12): p. 7499-509.

41. Harding, H.P., Y. Zhang, and D. Ron, Protein translation and folding are coupled by an endoplasmic-reticulum-resident kinase. Nature, 1999. 397(6716): p. 271-4.

42. Lu, P.D., H.P. Harding, and D. Ron, Translation reinitiation at alternative open reading frames regulates gene expression in an integrated stress response. J Cell Biol, 2004. 167(1): p. 27-33.

43. Yaman, I., et al., The zipper model of translational control: a small upstream ORF is the switch that controls structural remodeling of an mRNA leader. Cell, 2003. 113(4): p. 519-31. 
44. Harding, H.P., et al., An integrated stress response regulates amino acid metabolism and resistance to oxidative stress. Mol Cell, 2003. 11(3): p. 619-33.

45. Ron, D. and P. Walter, Signal integration in the endoplasmic reticulum unfolded protein response. Nat Rev Mol Cell Biol, 2007. 8(7): p. 519-29.

46. Schroder, M. and R.J. Kaufman, The mammalian unfolded protein response. Annu Rev Biochem, 2005. 74: p. 739-89.

47. Yamamoto, K., et al., Transcriptional induction of mammalian ER quality control proteins is mediated by single or combined action of ATF6alpha and XBP1. Dev Cell, 2007. 13(3): p. 365-76.

48. Timmins, J.M., et al., Calcium/calmodulin-dependent protein kinase II links ER stress with Fas and mitochondrial apoptosis pathways. J Clin Invest, 2009. 119(10): p. 2925-41.

49. Yao, S., et al., Activating transcription factor 6 mediates oxidized LDL-induced cholesterol accumulation and apoptosis in macrophages by up-regulating CHOP expression. J Atheroscler Thromb, 2013. 20(1): p. 94-107.

50. Fu, H.Y., et al., Ablation of C/EBP homologous protein attenuates endoplasmic reticulummediated apoptosis and cardiac dysfunction induced by pressure overload. Circulation, 2010. 122(4): p. 361-9.

51. McCullough, K.D., et al., Gadd153 sensitizes cells to endoplasmic reticulum stress by downregulating Bcl2 and perturbing the cellular redox state. Mol Cell Biol, 2001. 21(4): p. 1249-59.

52. Hoffstrom, B.G., et al., Inhibitors of protein disulfide isomerase suppress apoptosis induced by misfolded proteins. Nat Chem Biol, 2010. 6(12): p. 900-6.

53. Tu, B.P. and J.S. Weissman, Oxidative protein folding in eukaryotes: mechanisms and consequences. J Cell Biol, 2004. 164(3): p. 341-6.

54. Tu, B.P. and J.S. Weissman, The FAD- and O(2)-dependent reaction cycle of Ero1-mediated oxidative protein folding in the endoplasmic reticulum. Mol Cell, 2002. 10(5): p. 983-94.

55. Cuozzo, J.W. and C.A. Kaiser, Competition between glutathione and protein thiols for disulphidebond formation. Nat Cell Biol, 1999. 1(3): p. 130-5.

56. Mathers, J., et al., Antioxidant and cytoprotective responses to redox stress. Biochem Soc Symp, 2004(71): p. 157-76.

57. Zhang, D.D., Mechanistic studies of the Nrf2-Keap1 signaling pathway. Drug Metab Rev, 2006. 38(4): p. 769-89.

58. Cullinan, S.B., et al., Nrf2 is a direct PERK substrate and effector of PERK-dependent cell survival. Mol Cell Biol, 2003. 23(20): p. 7198-209.

59. Cullinan, S.B. and J.A. Diehl, PERK-dependent activation of Nrf2 contributes to redox homeostasis and cell survival following endoplasmic reticulum stress. J Biol Chem, 2004. 279(19): p. 20108-17.

60. Uehara, T., et al., S-nitrosylated protein-disulphide isomerase links protein misfolding to neurodegeneration. Nature, 2006. 441(7092): p. 513-7.

61. Townsend, D.M., et al., A glutathione S-transferase pi-activated prodrug causes kinase activation concurrent with S-glutathionylation of proteins. Mol Pharmacol, 2006. 69(2): p. 501-8.

62. Ellgaard, L. and E.M. Frickel, Calnexin, calreticulin, and ERp57: teammates in glycoprotein folding. Cell Biochem Biophys, 2003. 39(3): p. 223-47.

63. Ferrari, D.M. and H.D. Soling, The protein disulphide-isomerase family: unravelling a string of folds. Biochem J, 1999. 339 ( Pt 1): p. 1-10.

64. Xu, D., et al., Knockdown of ERp57 increases BiP/GRP78 induction and protects against hyperoxia and tunicamycin-induced apoptosis. Am J Physiol Lung Cell Mol Physiol, 2009. 297(1): p. L44-51.

65. Anathy, V., et al., Oxidative processing of latent Fas in the endoplasmic reticulum controls the strength of apoptosis. Mol Cell Biol, 2012. 32(17): p. 3464-78. 
66. Roberson, E.C., et al., Influenza induces endoplasmic reticulum stress, caspase-12-dependent apoptosis, and c-Jun $\mathrm{N}$-terminal kinase-mediated transforming growth factor-beta release in lung epithelial cells. Am J Respir Cell Mol Biol, 2012. 46(5): p. 573-81.

67. Hoffman, S.M., et al., Endoplasmic reticulum stress mediates house dust mite-induced airway epithelial apoptosis and fibrosis. Respir Res, 2013. 14: p. 141.

68. Zhao, G., H. Lu, and C. Li, Proapoptotic activities of protein disulfide isomerase (PDI) and PDIA3 protein, a role of the Bcl-2 protein Bak. J Biol Chem, 2015. 290(14): p. 8949-63.

69. Ono, Y., et al., A sigma-1 receptor antagonist (NE-100) prevents tunicamycin-induced cell death via GRP78 induction in hippocampal cells. Biochem Biophys Res Commun, 2013. 434(4): p. 904-9.

70. Osorio, F., B. Lambrecht, and S. Janssens, The UPR and lung disease. Semin Immunopathol, 2013. 35(3): p. 293-306.

71. Kim, S.R., et al., Endoplasmic reticulum stress influences bronchial asthma pathogenesis by modulating nuclear factor kappaB activation. J Allergy Clin Immunol, 2013. 132(6): p. 1397-408.

72. Pahl, H.L. and P.A. Baeuerle, Expression of influenza virus hemagglutinin activates transcription factor NF-kappa B. J Virol, 1995. 69(3): p. 1480-4.

73. Meyer, M., et al., Hepatitis B virus transactivator MHBst: activation of NF-kappa B, selective inhibition by antioxidants and integral membrane localization. EMBO J, 1992. 11(8): p. 29913001.

74. Martino, M.E., et al., Airway epithelial inflammation-induced endoplasmic reticulum Ca2+ store expansion is mediated by X-box binding protein-1. J Biol Chem, 2009. 284(22): p. 14904-13.

75. Kelsen, S.G., et al., Cigarette smoke induces an unfolded protein response in the human lung: $a$ proteomic approach. Am J Respir Cell Mol Biol, 2008. 38(5): p. 541-50.

76. Martino, M.B., et al., The ER stress transducer IRE1beta is required for airway epithelial mucin production. Mucosal Immunol, 2013. 6(3): p. 639-54.

77. Galindo, I., et al., The ATF6 branch of unfolded protein response and apoptosis are activated to promote African swine fever virus infection. Cell Death Dis, 2012. 3: p. e341.

78. Hetz, C., The unfolded protein response: controlling cell fate decisions under ER stress and beyond. Nat Rev Mol Cell Biol, 2012. 13(2): p. 89-102.

79. Hjelmqvist, L., et al., ORMDL proteins are a conserved new family of endoplasmic reticulum membrane proteins. Genome Biol, 2002. 3(6): p. RESEARCH0027.

80. Moffatt, M.F., et al., Genetic variants regulating ORMDL3 expression contribute to the risk of childhood asthma. Nature, 2007. 448(7152): p. 470-3.

81. Pozzan, T., et al., Molecular and cellular physiology of intracellular calcium stores. Physiol Rev, 1994. 74(3): p. 595-636.

82. Berridge, M.J., P. Lipp, and M.D. Bootman, The versatility and universality of calcium signalling. Nat Rev Mol Cell Biol, 2000. 1(1): p. 11-21.

83. Cantero-Recasens, G., et al., The asthma-associated ORMDL3 gene product regulates endoplasmic reticulum-mediated calcium signaling and cellular stress. Hum Mol Genet, 2010. 19(1): p. 111-21.

84. Bandyopadhyay, A., et al., Chemical chaperones assist intracellular folding to buffer mutational variations. Nat Chem Biol, 2012. 8(3): p. 238-45.

85. Yancey, P.H., Organic osmolytes as compatible, metabolic and counteracting cytoprotectants in high osmolarity and other stresses. J Exp Biol, 2005. 208(Pt 15): p. 2819-30.

86. Makhija, L., et al., Chemical chaperones mitigate experimental asthma by attenuating endoplasmic reticulum stress. Am J Respir Cell Mol Biol, 2014. 50(5): p. 923-31.

87. Kim, H.J., et al., Inhibition of endoplasmic reticulum stress alleviates lipopolysaccharide-induced lung inflammation through modulation of NF-kappaB/HIF-1alpha signaling pathway. Sci Rep, 2013. 3: p. 1142. 
88. Razani, B. and G. Cheng, NF-kappaB: much learned, much to learn. Sci Signal, 2010. 3(138): p. pe29.

89. Courtois, G. and T.D. Gilmore, Mutations in the NF-kappaB signaling pathway: implications for human disease. Oncogene, 2006. 25(51): p. 6831-43.

90. Gilmore, T.D., Introduction to NF-kappaB: players, pathways, perspectives. Oncogene, 2006. 25(51): p. 6680-4.

91. Ghosh, S. and M.S. Hayden, New regulators of NF-kappaB in inflammation. Nat Rev Immunol, 2008. 8(11): p. 837-48.

92. Basseres, D.S., et al., Requirement of the NF-kappaB subunit p65/RelA for K-Ras-induced lung tumorigenesis. Cancer Res, 2010. 70(9): p. 3537-46.

93. Hewson, C.A., et al., Rhinovirus induces MUC5AC in a human infection model, \& in vitro via NF$\{k a p p a\} B$ \& EGFR pathways. Eur Respir J, 2010.

94. Poynter, M.E., C.G. Irvin, and Y.M. Janssen-Heininger, Rapid activation of nuclear factor-kappaB in airway epithelium in a murine model of allergic airway inflammation. Am J Pathol, 2002.

160(4): p. 1325-34.

95. Janssen-Heininger, Y.M., et al., Nuclear factor kappaB, airway epithelium, and asthma: avenues for redox control. Proc Am Thorac Soc, 2009. 6(3): p. 249-55.

96. Reynaert, N.L., et al., Dynamic redox control of NF-kappaB through glutaredoxin-regulated Sglutathionylation of inhibitory kappaB kinase beta. Proc Natl Acad Sci U S A, 2006. 103(35): p. 13086-91.

97. Michalek, R.D., et al., The requirement of reversible cysteine sulfenic acid formation for $T$ cell activation and function. J Immunol, 2007. 179(10): p. 6456-67.

98. Sakai, J., et al., Reactive oxygen species-induced actin glutathionylation controls actin dynamics in neutrophils. Immunity, 2012. 37(6): p. 1037-49.

99. Adachi, T., et al., S-glutathiolation of Ras mediates redox-sensitive signaling by angiotensin II in vascular smooth muscle cells. J Biol Chem, 2004. 279(28): p. 29857-62.

100. Barrett, W.C., et al., Regulation of PTP1B via glutathionylation of the active site cysteine 215. Biochemistry, 1999. 38(20): p. 6699-705.

101. Nolin, J.D., et al., The glutaredoxin/S-glutathionylation axis regulates interleukin-17A-induced proinflammatory responses in lung epithelial cells in association with S-glutathionylation of nuclear factor kappaB family proteins. Free Radic Biol Med, 2014. 73: p. 143-53.

102. Finkel, T., Signal transduction by reactive oxygen species. J Cell Biol, 2011. 194(1): p. 7-15.

103. Evangelista, A.M., M.J. Kohr, and E. Murphy, S-nitrosylation: specificity, occupancy, and interaction with other post-translational modifications. Antioxid Redox Signal, 2013. 19(11): p. 1209-19.

104. Benhar, M., et al., Regulated protein denitrosylation by cytosolic and mitochondrial thioredoxins. Science, 2008. 320(5879): p. 1050-4.

105. Findlay, V.J., et al., A novel role for human sulfiredoxin in the reversal of glutathionylation. Cancer Res, 2006. 66(13): p. 6800-6.

106. Park, J.W., et al., Deglutathionylation of 2-Cys peroxiredoxin is specifically catalyzed by sulfiredoxin. J Biol Chem, 2009. 284(35): p. 23364-74.

107. Townsend, D.M., et al., Novel role for glutathione S-transferase pi. Regulator of protein SGlutathionylation following oxidative and nitrosative stress. J Biol Chem, 2009. 284(1): p. 436-45.

108. Terada, L.S., Specificity in reactive oxidant signaling: think globally, act locally. J Cell Biol, 2006. 174(5): p. 615-23.

109. Sevin, C.M., et al., Deficiency of gp91phox inhibits allergic airway inflammation. Am J Respir Cell Mol Biol, 2013. 49(3): p. 396-402. 
110. Banerjee, E.R. and W.R. Henderson, Jr., Role of T cells in a gp91phox knockout murine model of acute allergic asthma. Allergy Asthma Clin Immunol, 2013. 9(1): p. 6.

111. Banerjee, E.R. and W.R. Henderson, Jr., Defining the molecular role of gp91phox in the immune manifestation of acute allergic asthma using a preclinical murine model. Clin Mol Allergy, 2012. 10(1): p. 2.

112. Vulcano, M., et al., Toll receptor-mediated regulation of NADPH oxidase in human dendritic cells. J Immunol, 2004. 173(9): p. 5749-56.

113. Savina, A., et al., NOX2 controls phagosomal $\mathrm{pH}$ to regulate antigen processing during crosspresentation by dendritic cells. Cell, 2006. 126(1): p. 205-18.

114. Jancic, C., et al., Rab27a regulates phagosomal $\mathrm{pH}$ and NADPH oxidase recruitment to dendritic cell phagosomes. Nat Cell Biol, 2007. 9(4): p. 367-78.

115. Abdala-Valencia, H., et al., Nonhematopoietic NADPH oxidase regulation of lung eosinophilia and airway hyperresponsiveness in experimentally induced asthma. Am J Physiol Lung Cell Mol Physiol, 2007. 292(5): p. L1111-25.

116. Schroer, K.T., et al., Downregulation of glutathione S-transferase pi in asthma contributes to enhanced oxidative stress. J Allergy Clin Immunol, 2011. 128(3): p. 539-48.

117. van der Vliet, A., Nox enzymes in allergic airway inflammation. Biochim Biophys Acta, 2011. 1810(11): p. 1035-44.

118. Rada, B., et al., Histamine stimulates hydrogen peroxide production by bronchial epithelial cells via histamine $\mathrm{H} 1$ receptor and dual oxidase. Am J Respir Cell Mol Biol, 2014. 50(1): p. 125-34.

119. Hecker, L., et al., NADPH oxidase-4 mediates myofibroblast activation and fibrogenic responses to lung injury. Nat Med, 2009. 15(9): p. 1077-81.

120. Shelton, M.D. and J.J. Mieyal, Regulation by reversible S-glutathionylation: molecular targets implicated in inflammatory diseases. Mol Cells, 2008. 25(3): p. 332-46.

121. Reynaert, N.L., Glutathione biochemistry in asthma. Biochim Biophys Acta, 2011. 1810(11): p. 1045-51.

122. Fitzpatrick, A.M., et al., Glutathione oxidation is associated with airway macrophage functional impairment in children with severe asthma. Pediatr Res, 2011. 69(2): p. 154-9.

123. Fitzpatrick, A.M., et al., Heterogeneity of severe asthma in childhood: confirmation by cluster analysis of children in the National Institutes of Health/National Heart, Lung, and Blood Institute Severe Asthma Research Program. J Allergy Clin Immunol, 2011. 127(2): p. 382-389 e1-13.

124. Chan, R.C., et al., Pro-oxidative diesel exhaust particle chemicals inhibit LPS-induced dendritic cell responses involved in T-helper differentiation. J Allergy Clin Immunol, 2006. 118(2): p. 455-65.

125. Comhair, S.A., et al., Extracellular glutathione peroxidase induction in asthmatic lungs: evidence for redox regulation of expression in human airway epithelial cells. FASEB J, 2001. 15(1): p. 70-78.

126. Won, H.Y., et al., Glutathione peroxidase 1 deficiency attenuates allergen-induced airway inflammation by suppressing Th2 and Th17 cell development. Antioxid Redox Signal, 2010. 13(5): p. 575-87.

127. Dittrich, A.M., et al., Glutathione peroxidase-2 protects from allergen-induced airway inflammation in mice. Eur Respir J, 2010. 35(5): p. 1148-54.

128. Spiteri, M.A., et al., Polymorphisms at the glutathione S-transferase, GSTP1 locus: a novel mechanism for susceptibility and development of atopic airway inflammation. Allergy, 2000. 55 Suppl 61: p. 15-20.

129. Gilliland, F.D., et al., Effect of glutathione-S-transferase M1 and P1 genotypes on xenobiotic enhancement of allergic responses: randomised, placebo-controlled crossover study. Lancet, 2004. 363(9403): p. 119-25.

130. Zhou, J., et al., Glutathione transferase P1: an endogenous inhibitor of allergic responses in a mouse model of asthma. Am J Respir Crit Care Med, 2008. 178(12): p. 1202-10. 
131. Reynaert, N.L., E.F. Wouters, and Y.M. Janssen-Heininger, Modulation of glutaredoxin-1 expression in a mouse model of allergic airway disease. Am J Respir Cell Mol Biol, 2007. 36(2): p. 147-51.

132. Kuipers, I., et al., Increased glutaredoxin-1 and decreased protein S-glutathionylation in sputum of asthmatics. Eur Respir J, 2013. 41(2): p. 469-72.

133. Hoffman, S.M., et al., Genetic ablation of glutaredoxin-1 causes enhanced resolution of airways hyperresponsiveness and mucus metaplasia in mice with allergic airways disease. Am J Physiol Lung Cell Mol Physiol, 2012. 303(6): p. L528-38.

134. Lee, S., S.M. Kim, and R.T. Lee, Thioredoxin and thioredoxin target proteins: from molecular mechanisms to functional significance. Antioxid Redox Signal, 2013. 18(10): p. 1165-207.

135. Ito, W., et al., Thioredoxin in allergic inflammation. Int Arch Allergy Immunol, 2011. 155 Suppl 1: p. 142-6.

136. Torii, M., et al., Thioredoxin suppresses airway inflammation independently of systemic Th1/Th2 immune modulation. Eur J Immunol, 2010. 40(3): p. 787-96.

137. Imaoka, H., et al., Endogenous and exogenous thioredoxin 1 prevents goblet cell hyperplasia in a chronic antigen exposure asthma model. Allergol Int, 2009. 58(3): p. 403-10.

138. Ichiki, H., et al., Thioredoxin suppresses airway hyperresponsiveness and airway inflammation in asthma. Biochem Biophys Res Commun, 2005. 334(4): p. 1141-8.

139. Kobayashi, N., et al., Thioredoxin reduces C-C chemokine-induced chemotaxis of human eosinophils. Allergy, 2009. 64(8): p. 1130-5.

140. Hoshino, T., et al., Redox-active protein thioredoxin prevents proinflammatory cytokine- or bleomycin-induced lung injury. Am J Respir Crit Care Med, 2003. 168(9): p. 1075-83.

141. Brewer, J.P., A.B. Kisselgof, and T.R. Martin, Genetic variability in pulmonary physiological, cellular, and antibody responses to antigen in mice. Am J Respir Crit Care Med, 1999. 160(4): p. 1150-6.

142. Takeda, K., et al., Strain dependence of airway hyperresponsiveness reflects differences in eosinophil localization in the lung. Am J Physiol Lung Cell Mol Physiol, 2001. 281(2): p. L394-402.

143. Alcorn, J.F., et al., Strain-dependent activation of NF-kappaB in the airway epithelium and its role in allergic airway inflammation. Am J Physiol Lung Cell Mol Physiol, 2010. 298(1): p. L57-66.

144. Wilson, R.H., et al., Allergic sensitization through the airway primes Th17-dependent neutrophilia and airway hyperresponsiveness. Am J Respir Crit Care Med, 2009. 180(8): p. 720-30.

145. Wilson, R.H., et al., The Toll-like receptor 5 ligand flagellin promotes asthma by priming allergic responses to indoor allergens. Nat Med, 2012. 18(11): p. 1705-10.

146. Chapman, D.G., et al., Animal models of allergic airways disease: where are we and where to next? J Cell Biochem, 2014. 115(12): p. 2055-64.

147. Nelson, R.P., Jr., et al., Allergen-specific IgE levels and mite allergen exposure in children with acute asthma first seen in an emergency department and in nonasthmatic control subjects. J Allergy Clin Immunol, 1996. 98(2): p. 258-63.

148. McKinley, L., et al., TH17 cells mediate steroid-resistant airway inflammation and airway hyperresponsiveness in mice. J Immunol, 2008. 181(6): p. 4089-97.

149. Ano, S., et al., Transcription factors GATA-3 and RORgammat are important for determining the phenotype of allergic airway inflammation in a murine model of asthma. J Immunol, 2013. 190(3): p. 1056-65. 


\section{CHAPTER 2}

Endoplasmic reticulum stress mediates house dust mite-induced airway epithelial apoptosis and fibrosis

Sidra M. Hoffman, Jane E. Tully, James D. Nolin, Karolyn G. Lahue, Dylan H. Goldman, Nirav Daphtary, Minara Aliyeva, Charles G. Irvin, Anne E. Dixon, Matthew E. Poynter and Vikas Anathy

Respiratory Research 2013; 14. 141. 


\section{ABSTRACT}

Background: The endoplasmic reticulum (ER) stress response participates in many chronic inflammatory and autoimmune diseases. In the current study, we sought to examine the contribution of ER stress transducers in the pathogenesis of three principal facets of allergic asthma: inflammation, airway fibrosis, and airways hyperresponsiveness.

Methods: House Dust Mite (HDM) was used as an allergen for in vitro and in vivo challenge of primary human and murine airway epithelial cells. ER stress transducers were modulated using specific small interfering RNAs (siRNAs) in vivo. Inflammation, airway remodeling, and hyperresponsiveness were measured by total bronchoalveolar lavage (BAL) cell counts, determination of collagen, and methacholine responsiveness in mice, respectively.

Results: Challenge of human bronchiolar and nasal epithelial cells with HDM extract induced the ER stress transducer, activating transcription factor $6 \alpha$ (ATF6 $\alpha$ ) as well as protein disulfide isomerase, ERp57, in association with activation of caspase-3. SiRNA-mediated knockdown of ATF6 $\alpha$ and ERp57 during HDM administration in mice resulted in a decrease in components of HDM-induced ER stress, disulfide mediated oligomerization of Bak, and activation of caspase-3. Furthermore, siRNA-mediated knockdown of ATF6 $\alpha$ and ERp57 led to decreased inflammation, airway hyperresponsiveness and airway fibrosis.

Conclusion: Collectively, our work indicates that HDM induces ER stress in airway epithelial cells and that ATF6 $\alpha$ and ERp57 play a significant role in the development of cardinal features of allergic airways disease. Inhibition of ER stress responses may provide a potential therapeutic avenue in chronic asthma and sub-epithelial fibrosis associated with loss of lung function.

\section{Key words}

Allergen, HDM, unfolded protein response, ER stress, apoptosis, asthma, airway fibrosis 


\section{INTRODUCTION}

Airway inflammation and fibrosis impact lung structure and function in allergic asthma [1]. For instance, chronic asthmatics display extensive airway remodeling characterized by sub-epithelial fibrosis, goblet cell hyperplasia and increased thickness of the basement membrane [2-4]. To date, the processes that facilitate airway fibrosis in allergic asthma remain poorly understood and require a deeper understanding of the cellular and molecular responses to allergens in order to identify potential therapeutic targets.

House Dust Mite (HDM) is one of the most commonly found airborne allergens [5], inducing an allergic response in $50-85 \%$ of asthmatics [5, 6]. Extracts of HDM contain fungal spores, chitin, fecal pellets (containing proteases), Dermatophagoide (Der) family of proteins and lipopolysaccharide (LPS) [7-10]. Studies in rodents have shown that these components can activate multiple receptors present on airway epithelial cells, inducing the secretion of growth factors, the production of cytokines that regulate subsequent activation of T cells, mucus metaplasia, inflammation, airways hyperresponsiveness (AHR), and fibrosis $[5,11,12]$.

Physiological demand for increases in protein folding can create an imbalance in synthesis and capacity to fold. This leads to an increase in misfolded proteins in the endoplasmic reticulum (ER), initiating the ER stress response [13]. In mammalian cells, misfolded proteins are sensed by three ER transmembrane proteins: Inositol Requiring Enzyme 1 (IRE1), activating transcription factor 6 (ATF6), and PKR-like ER kinase (PERK) [14]. A prolonged unfolded protein response (UPR) can cause CCAAT/enhancer-binding protein (C/EBP) homologous protein (CHOP)-induced apoptosis [13]. Additionally, to cope with excessive protein folding load, the protein disulfide isomerases (PDIs), which construct disulfide bridge (-S-S-) formation in the ER, are upregulated [15]. One such PDI, ERp57, mediates misfolded protein-induced apoptosis by oligomerization of Bak through the formation of intermolecular disulfide (-S-S-) bridges and the permeabilization of mitochondria [16]. Studies thus far have investigated ER stress-dependent IRE1 signaling during mucus metaplasia in ovalbumin-induced allergic airway disease $[17,18]$. ER stress is known to play a prominent role in apoptosis of alveolar type II epithelial cells in Idiopathic Pulmonary Fibrosis (IPF) $[19,20]$ and Hermansky Pudlak Syndrome (HPS) [21]. It remains unknown whether ER stress responses are triggered by human asthma relevant allergens such as HDM. Furthermore, it is not clear whether allergen-induced airway epithelial ER stress 
and apoptosis are linked to sub-epithelial fibrosis and impairment in respiratory mechanics in a murine model of allergic airway disease.

The goal of the present study was to evaluate the impact of HDM, an asthma-relevant allergen, on ER stress responses, apoptosis in airway epithelial cells and subsequent effects on fibrosis and lung function. Our results demonstrate enhanced expression of ER stress transducers in murine and human epithelial cells in response to HDM challenge. In mice, airway epithelial ER stress was associated with up regulation of apoptotic and fibrotic markers after HDM exposure. In vivo siRNA mediated knockdown of ATF6 $\alpha$ and ERp57 attenuated inflammation and AHR, and abrogated airway fibrosis. These results indicate a critical role of airway epithelial ER stress in allergen-induced airway inflammation and fibrosis.

\section{MATERIALS AND METHODS}

Cell Culture, siRNA transfection and Caspase-3 assay. A human bronchial epithelial cell line (HBE) was kindly provided by Dr. Albert van der Vliet-University of Vermont, and cultured as described previously $[22,23]$ and primary human nasal epithelial cells were cultured as described previously [24]. Human cell lines were exposed to either PBS or $25 \mu \mathrm{g} / \mathrm{ml}$ of HDM (Greer, Lenoir, NC). All protocols that utilize primary human nasal epithelial cells were approved by the Institutional Review Board. Cells were transfected with plasmids or siRNA as described $[25,26]$. Caspase-3 activities were measured using Caspase-Glo 3 (Promega, Madison, WI) reagents, according to the manufacturer's protocol (Promega, Madison, WI). Results were expressed in Relative Luminescence Units (RLU), after subtraction of background luminescence values. Cell death was measured by MTT assay [25]. All results were obtained from 3 independent experiments conducted in triplicate.

HDM and OVA-LPS models of allergic airway disease. For all experiments, 8 to 12 wk old WT BALB/C mice (Jackson Laboratories) were used, as approved by the Institutional Animal Care and Use Committee. Mice ( $n=10 /$ group) were anesthetized with isofluorane and exposed to $50 \mu \mathrm{g}$ of the allergen, HDM (GREER-containing 35 endotoxin units/mg) extract, resuspended in PBS, via intranasal administration on day 0 and boosted again on day 7. Mice were then administered 50 $\mathrm{\mu g}$ of HDM consecutively on days $14-18$, and euthanized $48 \mathrm{~h}$ post final exposure. The control group was given $50 \mu \mathrm{l}$ of sterile PBS alone at all time points. Alternatively, mice were sensitized via oropharyngeal administration of $100 \mu \mathrm{g}$ of low endotoxin Ovalbumin (Grade V, Sigma Aldrich) in PBS with $0.1 \mu \mathrm{g}$ of LPS on days 0 and 7, challenged using 6 doses of aerosolized 1\% OVA in PBS for 30min on days 14-19, and 
euthanized on day 21. This protocol was adapted from a previously described method of airway sensitization and challenge [27].

SiRNA administration of ERp57 and ATF6 $\alpha$. Mice ( $\mathrm{n}=10 /$ group) were anesthetized with isofluorane and administered $10 \mathrm{mg} / \mathrm{kg}$ of scrambled small interfering (si) RNA or siRNA for ERp57 (Thermo ScientificL45187) and ATF6 (ORIGENE-SR418766) oropharyngeally on days $-1,6$, and 13, and again on days 16

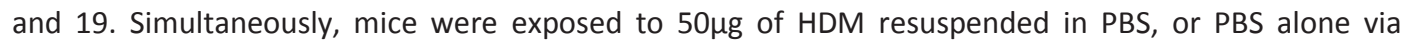
intranasal administration on days 1 and 7. Mice were then administered 50 $\mathrm{\mu g}$ of HDM consecutively, on days 14-18 and euthanized $72 \mathrm{~h}$ following the final HDM exposure. On day 16, when siRNA administration coincided with HDM exposure, mice received siRNA 6h prior to intranasal administration of HDM.

Assessment of AHR. Mice ( $n=10 /$ group) were anesthetized with an intraperitoneal injection of pentobarbital sodium $(90 \mathrm{mg} / \mathrm{kg})$, tracheotomized using an 18 gauge cannula, then mechanically ventilated at 200 breaths/min using a FlexiVent ${ }^{\mathrm{TM}}$ computer controlled small animal ventilator (SCIREQ). While on the ventilator mice also received the paralytic, pancuronium bromide. The parameters Newtonian resistance $(\mathrm{Rn})$, tissue damping $(\mathrm{G})$, and elastance $(\mathrm{H})$ were calculated as previously described $[28,29]$. Airway responsiveness is represented as the average of the 3 peak measurements for each animal, obtained at incremental methacholine doses.

Bronchoalveolar lavage processing. Bronchoalveolar lavage (BAL) from mice ( $n=10 /$ group) was collected. Total and differential cell counts were performed as previously described [20]. Briefly, cells were isolated by centrifugation and total cell counts were enumerated using the Advia 120 automated hematology analyzer system. Differential cell counts were obtained via cytospins using Hema3 stain reagents (Fisher Scientific). Differentials were performed on a minimum of 300 cells per animal.

Western blot analysis. Following dissection, right lung lobes were flash frozen for protein analysis. Lungs were pulverized, and lysed in buffer containing $137 \mathrm{mM}$ Tris $\cdot \mathrm{HCl}(\mathrm{pH} 8.0), 130 \mathrm{mM} \mathrm{NaCl}$, and 1\% NP-40. Proteins from cell lysates were prepared in the same buffer. Insoluble proteins were pelleted via centrifugation, and following protein quantitation of the supernatant, samples were resuspended in loading buffer with dithiothrietol (DTT), and resolved by SDS-PAGE. Proteins were transferred to PVDF and membranes were probed using a standard immunoblotting protocol using the following primary 
antibodies: P-IRE, IRE, GRP78, ATF6 ${ }^{50}$ and CHOP (Abcam), ERp57, GRP94 (Stressgen), Poly (ADP-ribose) polymerase (PARP) (BD Pharmingen) and $\beta$-actin (Sigma).

Non reducing gel electrophoresis. Lung homogenates were resuspended in loading buffer without the reducing agent dithiothrietol (DTT). A separate set of samples were resuspended in loading buffer with DTT to reduce the disulfide bonds. The samples were resolved by SDS-PAGE and subjected to western blot analysis.

Immunofluorescence. Following euthanization, left lobes were fixed with $4 \%$ paraformaldehyde, stored at $4^{\circ} \mathrm{C}$ overnight for fixation of the tissue, mounted in paraffin, and $5 \mu \mathrm{m}$ sections were affixed to glass microscope slides for histopathology as previously described [30]. Sections were prepared for immunofluorescence by deparaffinizing with xylene and rehydrating through a series of ethanols [30]. For antigen retrieval, slides were heated for $20 \mathrm{~min}$ in $95^{\circ} \mathrm{C}$ citrate buffer $(\mathrm{pH} \mathrm{6.0)}$ ) with $0.05 \%$ TWEEN-20 then rinsed in distilled water. Sections were then blocked for $1 \mathrm{~h}$ in $1 \%$ bovine serum albumin (BSA) in PBS, followed by incubation with primary antibody for ERp57 (Stressgen), and Caspase-3 (Cell Signal) at 1:500, overnight at $4^{\circ} \mathrm{C}$. Slides were then washed $3 \times 5 \mathrm{~min}$ in PBS, incubated with Alexafluor 647 at 1:1000 in 1\% BSA, and counterstained with DAPI in PBS at 1:4000 for nuclear localization. Sections were imaged using a Zeiss 510-META confocal laser scanning microscope.

Measurement of collagen and immunohistochemistry. Collagen content was measured via the Sircol assay ( $n=10 /$ group) (Biocolor Ltd, UK). Briefly, lung lobes were diced and placed in $500 \mu \mathrm{l}$ of $10 \mathrm{mg} / \mathrm{mL}$ pepsin in $0.5 \mathrm{M}$ acetic acid for $3 \mathrm{~h}$ at $37^{\circ} \mathrm{C}$, or until lungs were completely digested. The digest was spun at $10,000 \mathrm{~g}$ for $10 \mathrm{~min}$ at room temperature. Fifty microliters of the supernatant was mixed vigorously with $500 \mu \mathrm{L}$ of sircol dye solution for $30 \mathrm{~min}$ and then spun again at $10,000 \mathrm{~g}$ for $10 \mathrm{~min}$. Excess dye was decanted off, and the resulting pellet was dissolved in $500 \mu \mathrm{L}$ of an alkaline solution, $200 \mu \mathrm{L}$ of which was pipetted in duplicates into a 96 well plate and measured at $540 \mathrm{~nm}$. To evaluate regional changes in alpha-smooth muscle actin ( $\alpha S M A)$, fixed sections were prepared for immunostaining by deparaffinizing with xylene and rehydrating through a series of ethanols. For antigen retrieval, slides were heated for $20 \mathrm{~min}$ in $95^{\circ} \mathrm{C}$ citrate buffer ( $\mathrm{pH} \mathrm{6.0)}$ ), then rinsed in distilled water. Sections were then blocked for $1 \mathrm{~h}$ in blocking serum as per manufacturer's instructions (Vectastain Alkaline Phosphatase Universal, Vector). Slides were then washed in TBS with $0.1 \%$ TWEEN-20 3x5min, followed by incubation with primary antibody for aSMA (Sigma) overnight at $4^{\circ} \mathrm{C}$. Sections were washed again and incubated with a 
biotinylated universal secondary antibody (Vectastain Alkaline Phosphatase Universal, Vector) for 30min at room temperature. Slides were washed and incubated with the Vectastain ABC-AP reagent (prepared as per manufacturer's instructions) for $30 \mathrm{~min}$ at room temperature. Sections were then incubated with Vector Red Alkaline Phosphatase Substrate Kit I (Vector) for 10min at room temperature, rinsed with tap water, and counterstained with Mayer's Hemotoxylin.

RNA isolation and mRNA assessment. Flash frozen right lobes of lungs were homogenized in liquid nitrogen and RNA was extracted, DNAse treated using RNEasy columns (QIAgen) and reverse transcribed into cDNA using MMLV (Gibco-BRL) according to manufacturer's instructions. Inflammatory gene expression was determined by semi-quantitative RT-PCR using an Applied Biosystem CFX1000 with 25ng of cDNA per reaction and SYBR green for individual target genes. The following forward and reverse primers were used to amplify the IL-13 (Fwd:5'-CCAGGCCCCTTCTAATGA-3', Rv:5'GCCTCTCCCCAGCAAAGTCT-3') and IFNy (Fwd:5'-GCGTCATTGAATCACACCTG-3' Rv:5'ACCTGTGGGTTGTTGACCTC-3"). The fold induction was normalized using the housekeeping gene cyclophilin.

Densitometric Analysis. Densitometric analyses were performed utilizing BioRad ${ }^{\circledR}$ VersaDoc $^{\mathrm{TM}}$ Imager Software (Minneapolis, MN). Briefly, Western Blot x-ray films were imaged and rectangles of identical size were traced around each band and assessed for density (intensity/ $/ \mathrm{mm}^{2}$ ). Values were normalized to corresponding $\beta$-Actin or total IRE bands following background subtraction.

Statistics. All assays were performed in triplicates. Data were analyzed by one-way analysis of variance (ANOVA) using the Tukey's test to adjust for multiple comparisons or student's t test where appropriate. Histopathological scores were analyzed using the Kruskal-Wallis test and Dunn's multiple comparison post hoc tests. Data from multiple experiments were averaged and expressed as mean values \pm SEM.

\section{RESULTS}

HDM induces ER stress and death in human epithelial cells. HDM is a complex allergen known to activate multiple receptors and their consequent downstream pathways [5]. In the current study, we hypothesized that these events would result in increased ER stress in epithelial cells. To address this hypothesis, primary human nasal epithelial (PHNE) cells from two non-asthmatic subjects and a human bronchial epithelial (HBE) cell line were challenged with either HDM or PBS as a control. The optimal 
dose of $25 \mu \mathrm{g} / \mathrm{ml}-\mathrm{HDM}$ was selected based on our prior analysis in the laboratory, which showed induction of inflammatory as well as robust ER stress responses in lung epithelial cells (data not shown). Seventy-two hours following repeated challenge, both subjects exhibited increases in phosphorylation of IRE1 (P-IRE), albeit to a greater extent in cells from subject 2, as well as increases in ER chaperone GRP78 (Bip), GRP94, and ERp57. ER stress transducer-ATF6 $\alpha$ and downstream transcriptional effector CHOP were also increased after HDM exposure (Fig 1A). With the exception of P-IRE, HBE cells responded in a similar manner with slight differences in kinetics between members of the ER stress responders (Fig. 1A). As previously shown, physiological processes demanding a high rate of protein synthesis and secretion may lead to unresolved ER stress resulting in apoptosis [14]. Accordingly, allergen exposure resulted in significant activation of caspase- 3 at varying levels in both PHNE and HBE cells (Fig. 1B).

During ER stress, activation of ATF6 $\alpha$ is known to specifically up regulate PDIs, chaperones, as well as CEBP homologous protein $\mathrm{CHOP}$, and consequently, these events are known to lead to apoptosis $[13,31]$. To address the contribution of ATF6 $\alpha$ activation, chaperone induction, and downstream activation of apoptosis, HBE cells were transfected with either scrambled small interfering (si) RNA (Siscr) or siRNA for ATF6 $\alpha$. Twenty-four hours following transfection, cells were stimulated with $25 \mu \mathrm{g}$ of HDM or PBS and harvested at 48 and 72h after exposure. Knockdown of ATF6 $\alpha$ in HBE cells resulted in decreased activation of the 50kD fragment of ATF6 $\alpha$, CHOP, and ERp57 in whole cell lysates, indicating a requirement for ATF6 in HDM-driven expression of CHOP and ERp57 (Fig. 1C). Following HDM administration, active caspase- 3 was increased and cell survival was decreased in scrambled siRNA transfected HBE cells. Knockdown of ATF6 $\alpha$ in HBE cells showed significant decrease in caspase-3 activity and an increase in cell survival (Fig. 1D and E) in cell treated with HDM. These results indicate that allergen (HDM) exposure can induce ER stress, and in turn, lead to apoptosis in human lung epithelial cells. 

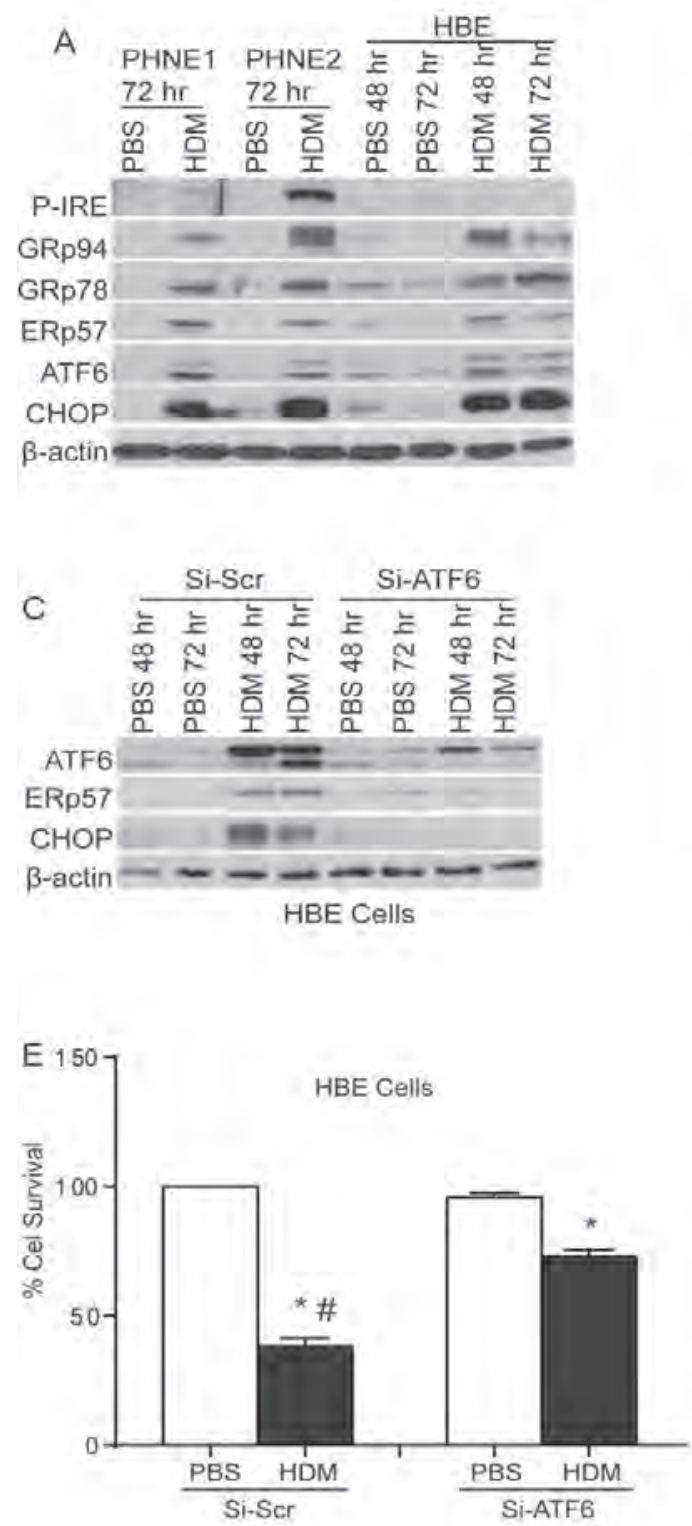
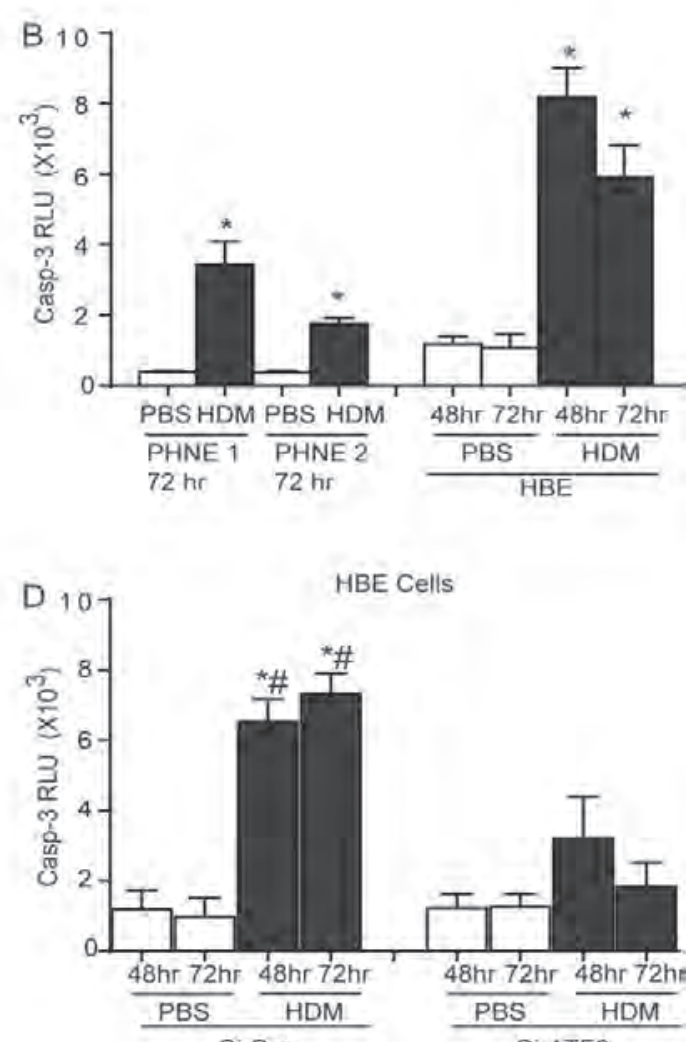

Si-Scr

Si-ATFG

Figure 1. HDM induces ER stress and activation of caspase-3 in primary human nasal (PHNEs) and bronchial epithelial cells (HBE). PHNEs from two subjects and HBEs were treated with HDM for the indicated time. The cell lysates were subjected to western blot analysis to detect ER stress markers (A). Caspase-3 activity was measured using a luminescence assay (B). * indicates $p<0.05$ as compared to their PBS controls by ANOVA from 2 experiments in triplicate. HBE cells were transfected with siRNA for ATF6 $\alpha$ or a non-specific Scr sequence, challenged with HDM, and subjected to western blot analysis to detect ER stress markers (C). Caspase-3 activity was measured using a luminescence assay (D). Cell death was quantified using MTT assay (E). * indicates $p<0.05$ as compared to their respective PBS controls. \# indicates $p<0.05$ as compared to their siRNA transfected HDM challenged samples (by ANOVA). 
HDM induces a robust ER stress response and apoptosis in mouse airway epithelial cells in vivo. To elicit allergic airways disease we challenged mice with a ubiquitous allergen-HDM, or Ovalbumin and compared the responses with mice treated with LPS (a model of acute lung injury and inflammation). Mice were initially sensitized and challenged via intranasal administration of HDM, LPS or low endotoxin OVA with $0.1 \mu \mathrm{g}$ of LPS (as an adjuvant) and were euthanized on day 21 [27] (Fig. 2A). Results in figures 2B and S1 demonstrates activation of ER stress in response to HDM or OVA/LPS in the whole lung, as evidenced by increases in phosphorylation of IRE1, as well as increased expression of GRP78, GRP94 and ERp57. ATF6 $\alpha$ and CHOP were also increased after HDM exposure as compared to controls. In contrast to our observation in the HDM model, ATF6 $\alpha$ did not appear to increase after OVA/LPS, but we observed a slight elevation in GRP94 and CHOP expression (Fig 2B and S1). Analysis of inflammatory cells showed a significant increase in eosinophils and lymphocytes in both models as compared to controls (Table 1). Macrophages were decreased in HDM challenged mice as compared to PBS controls, while in LPS and OVA/LPS challenged mice there was a significant increases in macrophages (Table 1). Immunofluorescence of HDM-instilled lungs indicated increased ERP57 as well as active caspase-3 predominantly in the bronchiolar epithelium, as compared to controls (Fig. 2C). Collectively these results demonstrate that HDM is a potent activator of ER stress in murine models of allergic airway disease, in comparison to Ovalbumin. Based on these results we continued our evaluations of the role of ER stress in allergic airways disease using the HDM model. 

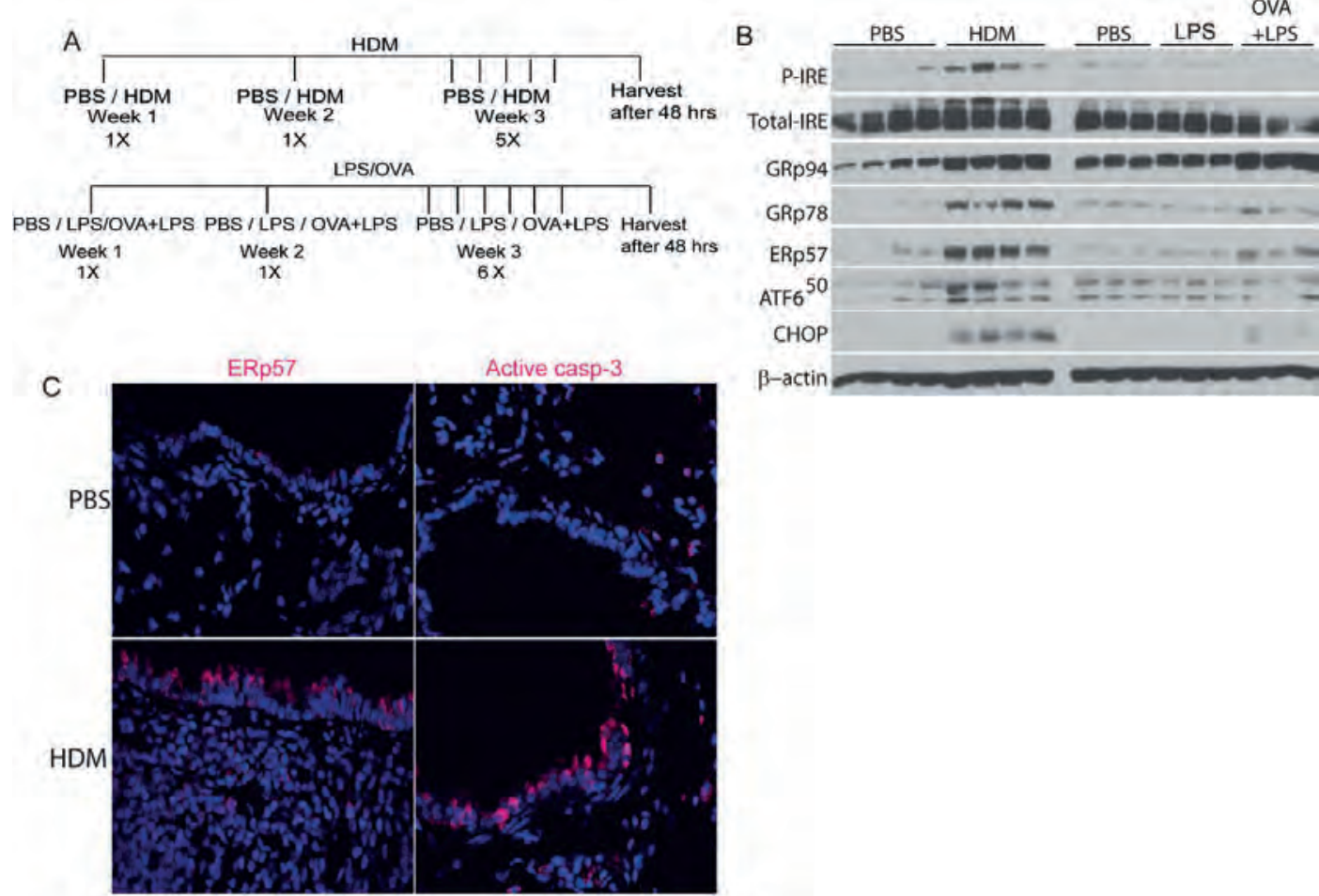

Table 1. Inflammatory profiles of models of allergic airway disease as depicted in A.

*indicates $p<0.05$ as compared to their respective PBS controls.

\# indicates $p<0.05$ as compared to their respective LPS treated mice.

BAL Cell Differentials

\begin{tabular}{rccccc}
\hline$\times 10^{3}$ & PBS & HDM & PBS & LPS & OVALPS \\
\hline MACS & $43.6 \pm 5.0$ & $21.4 \pm 5.0^{*}$ & $27.2 \pm 4.9$ & $40.6 \pm 6.6^{*}$ & $100.5 \pm 16.4^{*} \#$ \\
EOS & $0.7 \pm 0.4$ & $189.4 \pm 26.3^{*}$ & $0.0 \pm 0.0$ & $0.1 \pm 0.1$ & $51.2 \pm 10.3^{*} \#$ \\
PMN & $1.3 \pm 1.2$ & $1.4 \pm 0.2$ & $0.2 \pm 0.0$ & $0.1 \pm 0.1$ & $21.5 \pm 6.0^{*} \#$ \\
LYMPH & $0.7 \pm 0.3$ & $15.7 \pm 2.1^{*}$ & $1.6 \pm 0.6$ & $3.0 \pm 0.9$ & $29.8 \pm 7.1^{*} \#$
\end{tabular}

Figure 2. HDM induces ER stress and active caspase-3. Mice were challenged with PBS, HDM, LPS or Ovalbumin+LPS as depicted (A). Western blot analysis of whole lung lysates for ER stresses markers (B). Representative images showing up-regulation of ERp57 and active caspase-3 in the airway epithelium of HDM challenged mice $(n=4)(C)$. 

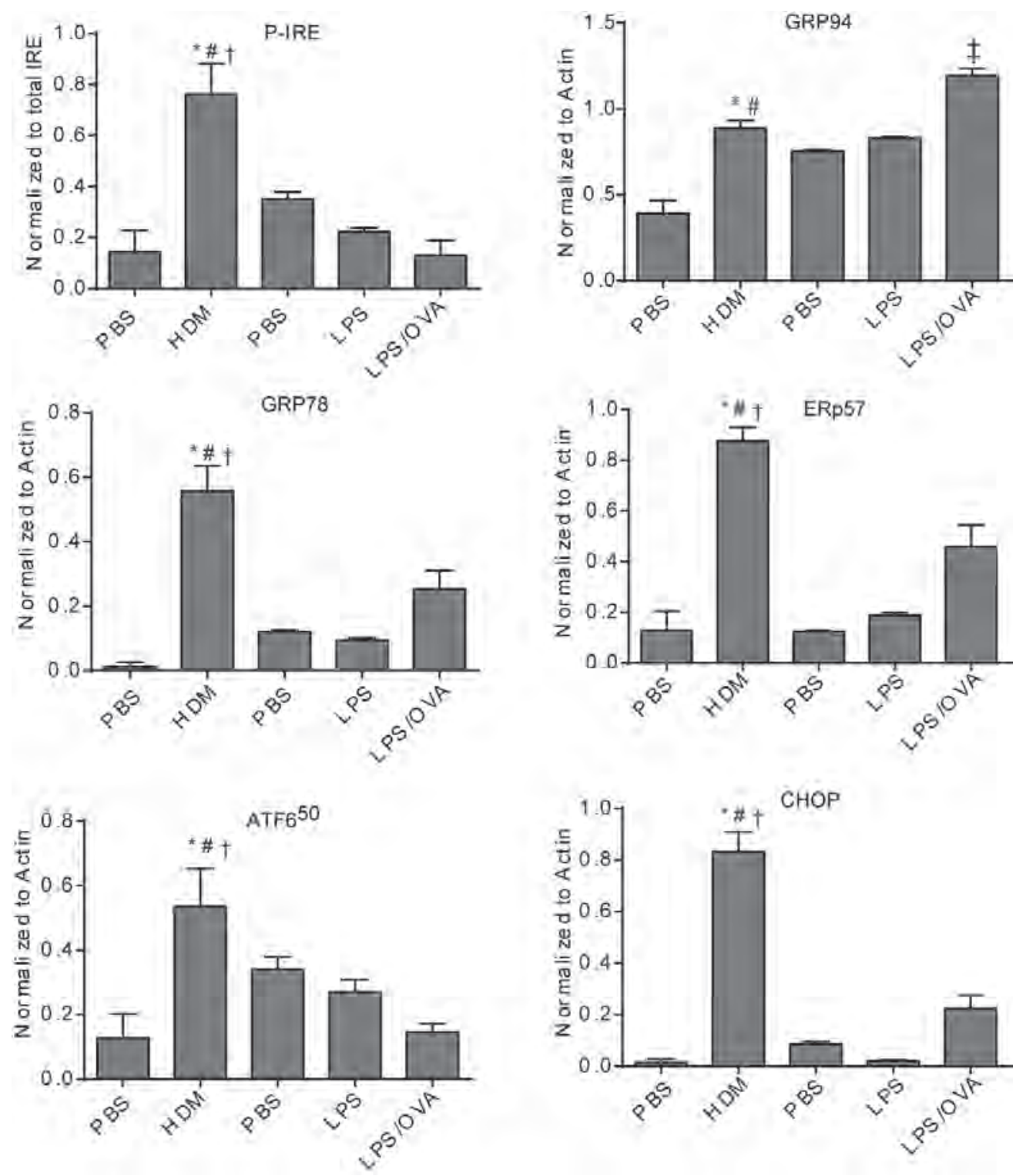

Supplementary Figure 1. Quantitaion of bands on the western blots in figure 2B by densitometry. All samples were normalized to actin except P-IRE.* indicates $p<0.05$ as compared to PBS controls, \# indicates $p<0.05$ as compared to their LPS and + indicates $p<0.05$ as compared to LPS/OVA samples by ANOVA. In the bar graph for GRP94 ‡ indicates $p<0.05$ as compared to HDM samples.

Knockdown of ATF6 $\alpha$ and ERp57 decreases CHOP, Bak oligomerization, and apoptosis in mice. To evaluate the role of ATF6 $\alpha$ and ERp57 in HDM-induced allergic airways disease in vivo, mice were 
administered $10 \mathrm{mg} / \mathrm{kg}$ oropharyngeal scrambled siRNA (Si-scr) or siRNA targeting ERp57 and ATF6 $\alpha$. Subsequently, the mice were challenged via the intranasal administration with $50 \mu \mathrm{g}$ of HDM consecutively on days 15-19 and euthanized 72h after final challenge (Fig. 3A). As expected, protein expression of ERp57 and ATF6 $\alpha$ in the whole lung was decreased after repeated HDM exposure in mice that received siRNA for ERp57 and ATF6 $\alpha$ (Si-ERp57+ATF6) compared to siRNA control samples (Si-scr). We also observed decreases in CHOP and GRP78 in HDM challenged mice treated with Si-ERp57+ATF6 $\alpha$ as compared to Si-scr. The elevated expression of GRP94 was not altered following HDM challenge and treatment with Si-ERp57+ATF6 a relative to Si-scr HDM groups (Fig. 3B and S2), demonstrating that not all ER stress responders were altered by knockdown of ERp57 and ATF6 $\alpha$. ER stress-induced ERp57 is known to form disulfide (-S-S-) bridges in proapoptotic Bak [16]. Results in figures $3 C$ and S2 demonstrate that siRNA-mediated knockdown of ERp57 and ATF6 $\alpha$ resulted in significantly decreased S-S- mediated oligomerization of Bak, manifested by decreases in the 75 and 50 kD forms of Bak. Furthermore, we also observed that caspase-3 activity and Poly (ADP-ribose) Polymerase (PARP), a target for caspase-3 cleavage during apoptosis, was significantly decreased in the whole lung lysates after ATF6 $\alpha$ and ERp57 knockdown following HDM challenge compared to Si-scr controls (Fig. 3D and E). This indicates that knockdown of ATF6 $\alpha$ and ERp57 results in significant decrease in HDM-induced, ER stress-mediated apoptotic cascade in the lung. 

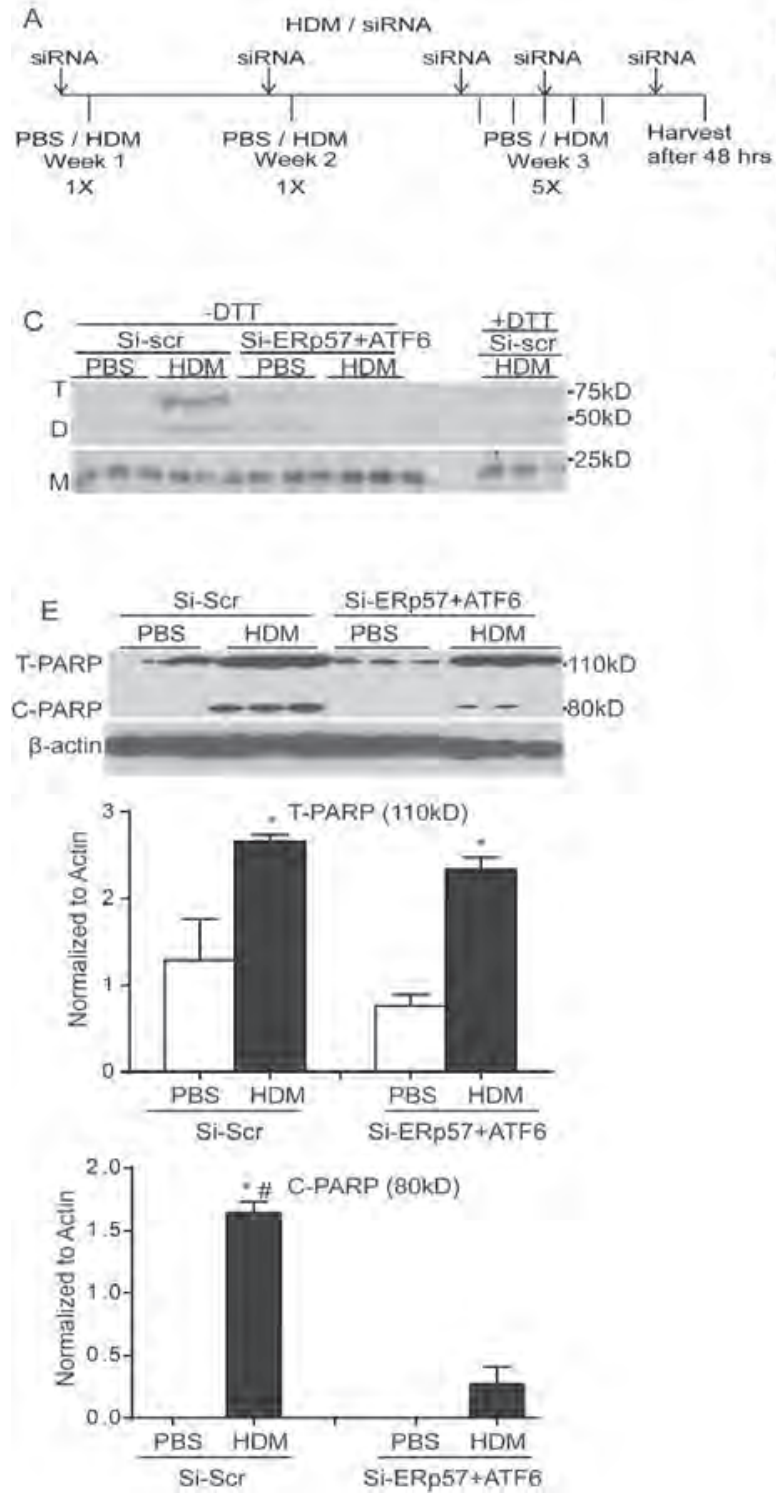

Figure 3. SiRNA-mediated knockdown of ATF6 $\alpha$ and ERp57 decreases -S-S- Bak oligomerization and active caspase-3. Mice were challenged with HDM and treated with scrambled siRNA (Si-Scr) or ERp57+ ATF6 $\alpha$ siRNA (SiERp57+ ATF6 $\alpha$ ) (A). Western blot analysis of whole lung lysates for ER stress markers post siRNA and HDM treatment (B). Disulfide -S-S- mediated oligomerization status of Bak post siRNA treatment (+DTT samples were used as controls) (C). Caspase-3 activity was measured in whole lung lysates using a luminescence assay (D). Cleavage of PARP from whole lung lysates were measured by western blots (T-PARP=total PARP, C-PARP=cleaved PARP) (E) and the bar graphs below represent quantitation of total and cleaved PARP by densitometry. * indicates $p<0.05$ as compared to their respective PBS controls. \# indicates $p<0.05$ as compared to their siRNA transfected HDM challenged samples by ANOVA. 

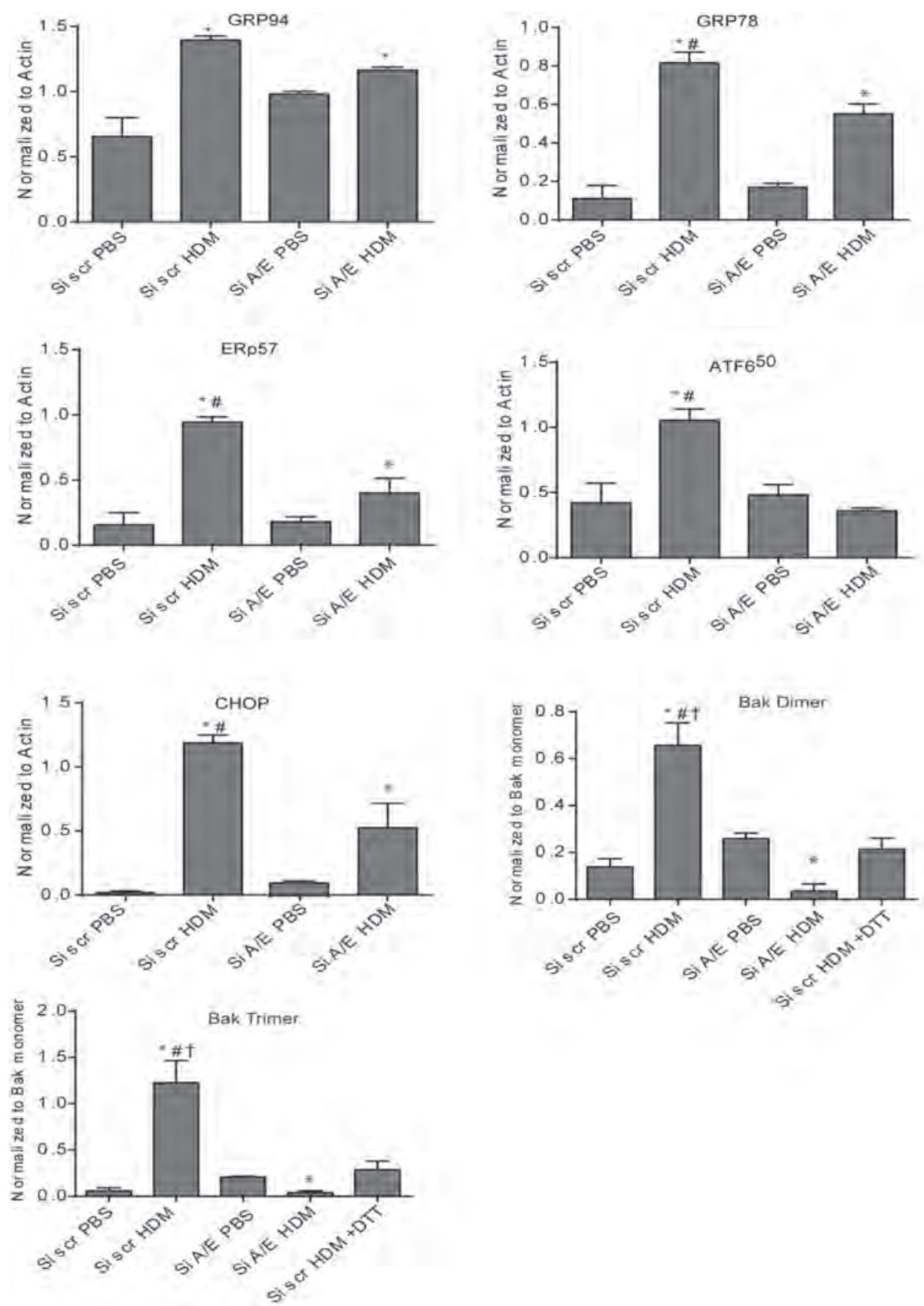

Supplementary Figure 2. Quantitaion of bands in the western blots in figure 3B and 3C by densitometry. All samples were normalized to actin.* indicates $p<0.05$ as compared to PBS controls, \# indicates $p<0.05$ as compared to their HDM samples and + indicates $p<0.05$ as compared to DTT treated samples by ANOVA. 
Knockdown of ERp57 and ATF6a partially decreases airway inflammation and airway hyperresponsiveness (AHR). As expected, animals in the Si-scr or Si-ERp57+ATF6 groups that were not exposed to HDM exhibited primarily macrophages in BALF. However, mice that received Si-scr and were challenged with HDM showed a marked influx of cells into the airways, characterized by increases in eosinophils, lymphocytes, and to a lesser extent, neutrophils. In contrast, knockdown of ERP57 and ATF6 $\alpha$ resulted in decreases in HDM-induced eosinophils and lymphocytes (Fig. 4A, B, C, and D). As expected, we observed significant increases in IL-13 mRNA in HDM challenged mice as compared to PBS controls. Additionally, there was no significant increase in IFN $\gamma$ mRNA (Fig S3), indicating that instillation of double stranded siRNA did not alter HDM-induced responses from Th2 to Th1. To address the functional effects of siRNA-mediated knockdown of ERp57 and ATF6 $\alpha$ in the airways, a forced oscillation technique was used to evaluate alterations in respiratory mechanics $[28,29]$ in response to HDM challenge. We did not observe any significant differences in central airway resistance $\left(R_{n}\right)$ at either the lowest or highest dose of methacholine in HDM-challenged siRNA-treated groups of mice, but we did observe significant increases in $\mathrm{R}_{\mathrm{n}}$ in mice that received Si-ERp57+ATF6 $\alpha$ as compared to Si-scr, after HDM challenge (Fig. 5A). In the peripheral airways, tissue resistance/dampening (G) and elastance/stiffness $(H)$ were significantly decreased in mice that received Si-ERp57+ATF6 $\alpha$ as compared to Si-scr, after HDM challenge (Fig. 5B and C). Although our analysis revealed an increase in Muc5AC mRNA levels after HDM challenge, there were no significant differences in levels of Muc5AC mRNA or Periodic Acid Schiff (PAS) staining in HDM-challenged ERp57+ATF6a-siRNA treated groups (data not shown). 
A

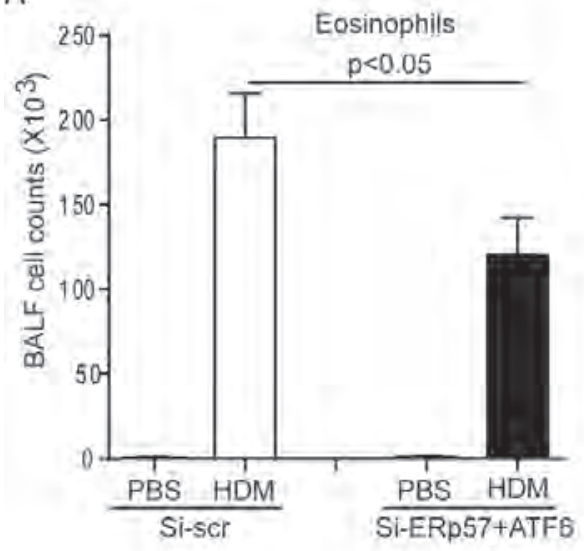

C

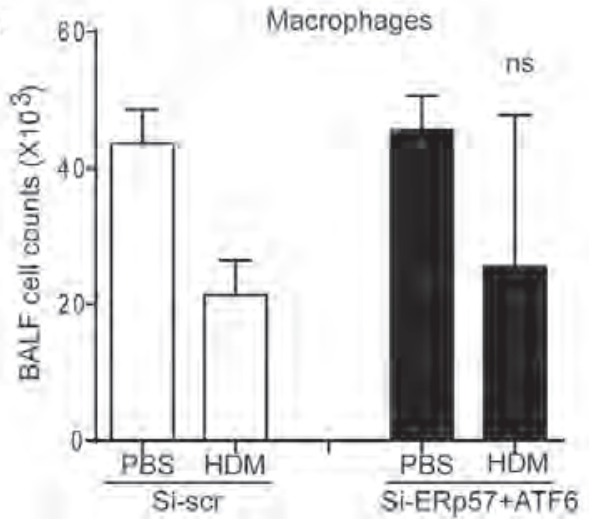

B
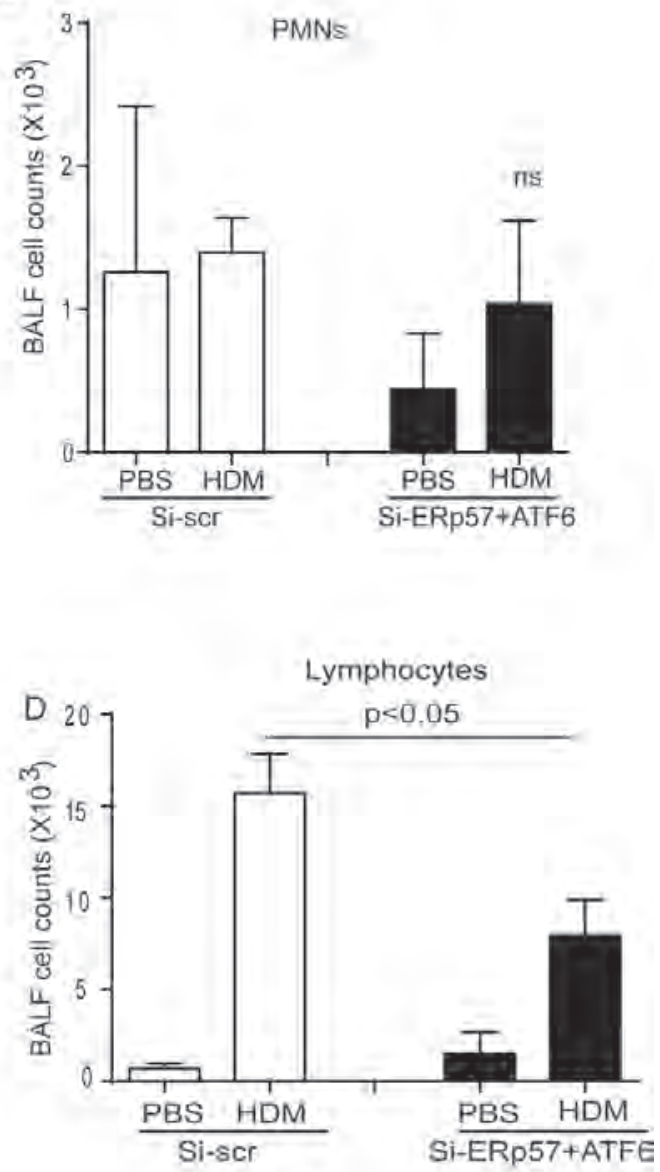

Figure 4. SiRNA-mediated knockdown of ATF6 $\alpha$ and ERp57 partially decreases HDM-induced eosinophils and lymphocytes in BALF. Mice were challenged with HDM and treated with siRNA as depicted in Fig 3A. BALF fluid was collected from mice ( $n=10$ /group) and eosinophils (A), PMNs (B), macrophages (C) and lymphocytes (D) were counted using a hemocytometer. $\mathrm{p}<0.05$ as measured by ANOVA, ns= not significant. 

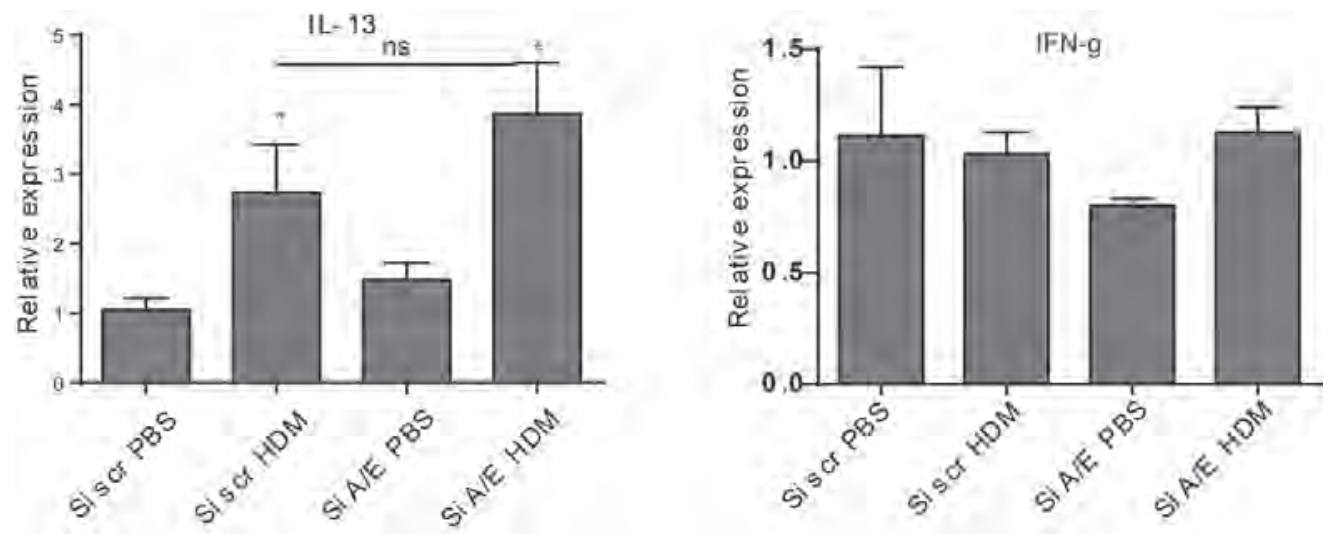

Supplementary Figure 3. Measurement of cytokines in the whole lung lysates. IL-13 and IFN $\gamma$ mRNA levels were measured by qRT-PCR. * indicates $\mathrm{p}<0.05$ as compared to PBS controls, ns-not significant. 

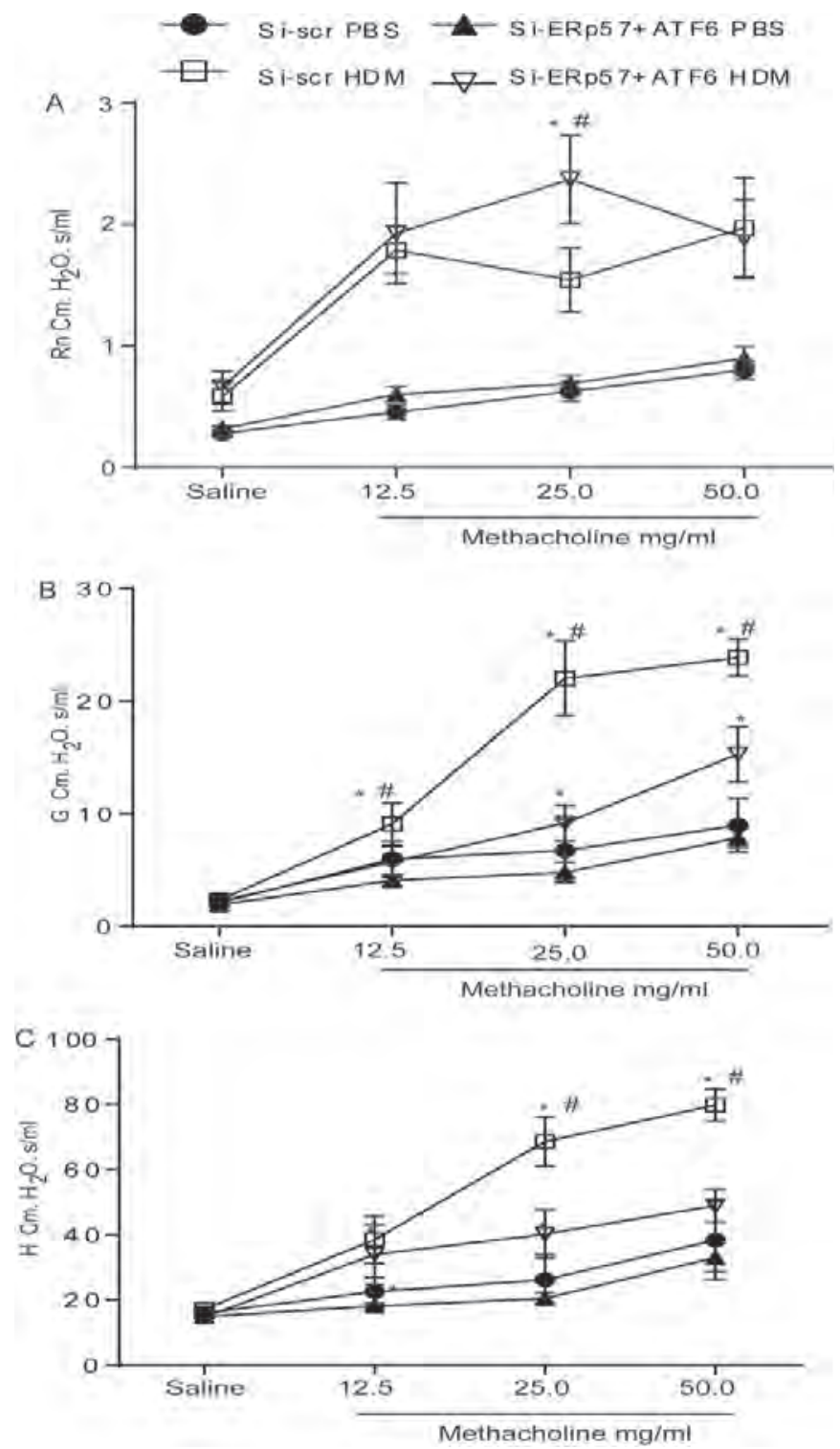

Figure 5. SiRNA mediated knockdown of ATF6 $\alpha$ and ERp57 decreases HDM-induced airway hyperresponsiveness.

Assessment of airway mechanics using a forced oscillation technique. Measurement of Newtonian Resistance $\left(R_{n}\right)$ $(A)$, airflow heterogeneity or tissue resistance $(G)(B)$, and airway closure/elastance $(H)(C)$, in response to varying doses of methacholine. ${ }^{*} \mathrm{p}<0.05$ by ANOVA, denotes differences in peak responses, compared with PBS controls. \# $p<0.05$ by ANOVA, denotes differences in peak responses, compared with the HDM groups ( $n=10$ mice/group). 
SiRNA mediated knockdown of ERp57 and ATF6 $\alpha$ decreases airway fibrosis. To examine the role of ATF6 $\alpha$ and ERp57 in airway remodeling, collagen deposition was evaluated following siRNAadministration and HDM exposure. Analysis of deposition of collagen by Masson's Trichrome in mice receiving HDM and Si-scr exhibited significant increases over PBS controls, whereas animals that were treated with HDM and received si-ERp57+ATF6 $\alpha$ showed decreases in Masson's trichrome staining (Fig. 6A) as compared to HDM treated, Si-scr animals. Semi quantitative scoring for Masson's trichrome staining by three independent scientists blinded to the identity of the samples revealed significant decreases in HDM challenged si-ERp57+ATF6 $\alpha$ treated mice (Fig. 6B) over HDM Si-scr treated animals. Additionally, alpha-smooth muscle actin ( $(S M A)$ was increased in the peribronchiolar region of HDM challenged mice treated with Si-scr (Fig 6C) as compared to HDM challenged si-ERp57+ATF6 $\alpha$ treated mice. Western blot analysis of whole lung lysates also showed a significant increase in $\alpha \mathrm{SMA}$ and Fibroblast Specific Protein (FSP-1) in HDM challenged mice treated with Si-scr (Fig 6D and S4). These increases were attenuated in mice challenged with HDM and treated with si-ERp57+ATF6 $\alpha$ (Fig 6D and S4). Furthermore, we also observed an increase in total collagen content in whole lung lysates from HDM challenged mice treated with Si-scr as compared to mice challenged with HDM and treated with siERp57+ATF6 $\alpha$ (Fig 6E). Collectively these results indicate that ER stress mediators act to control allergen induced airway fibrosis in the lung. 

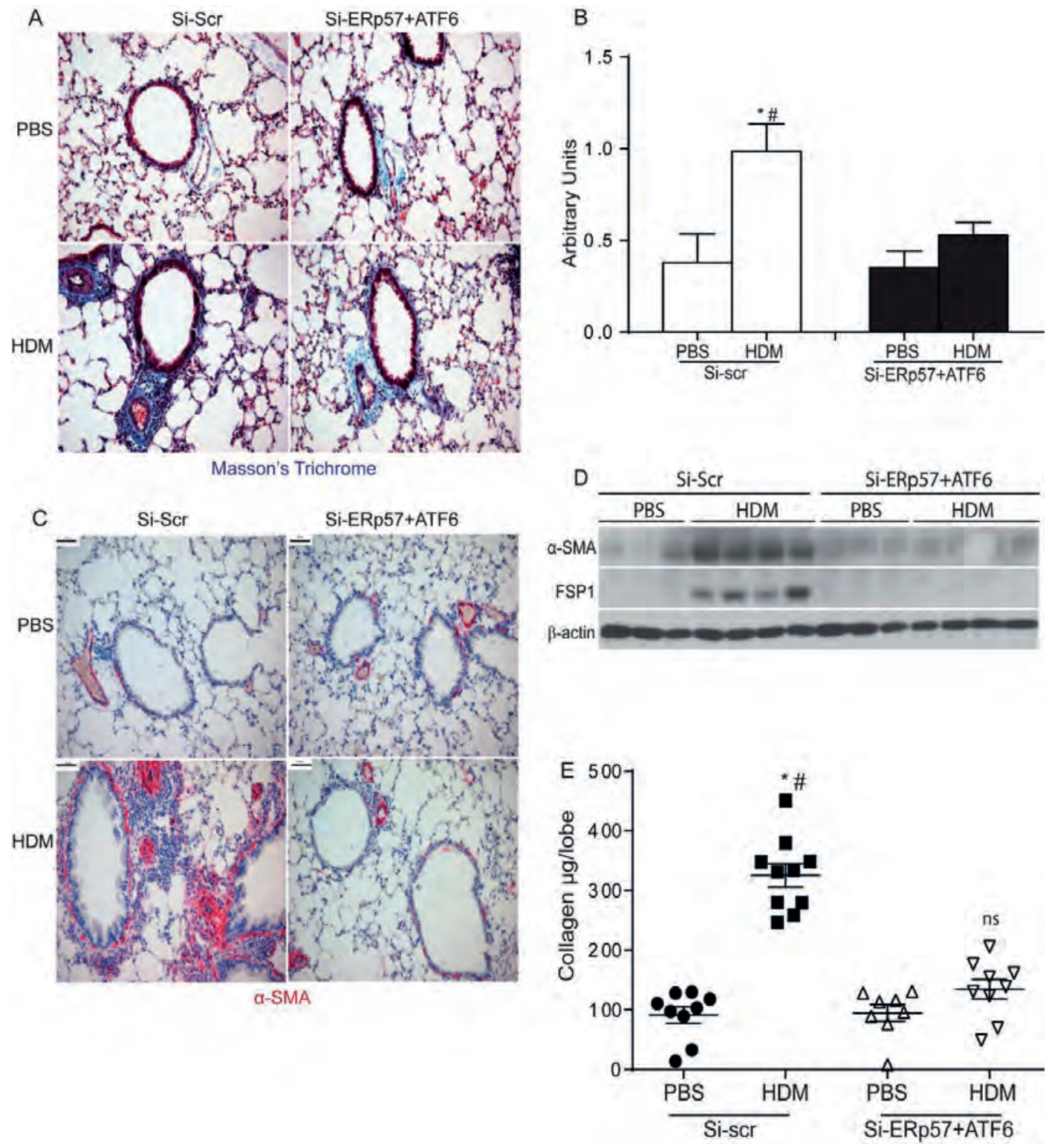

Figure 6. SiRNA mediated knockdown of ATF6 $\alpha$ and ERp57 decreases HDM-induced airway fibrosis. Representative images and scoring for airways stained with Masson's trichrome (A \& B). * indicates $p<0.05$ as compared to their respective PBS controls. \# indicates $p<0.05$ as compared to their siRNA transfected HDM challenged samples. Representative images stained with an antibody for $\alpha$ SMA (C). Western blot analysis of whole lung lysates for $\alpha$ SMA, FSP1 and $\beta$-actin (D). Measurement of collagen (E), * indicates $p<0.05$ as compared to their respective PBS controls. \# indicates $p<0.05$ as compared to their siRNA transfected HDM challenged samples, ns= not significant compared to PBS treated samples. 

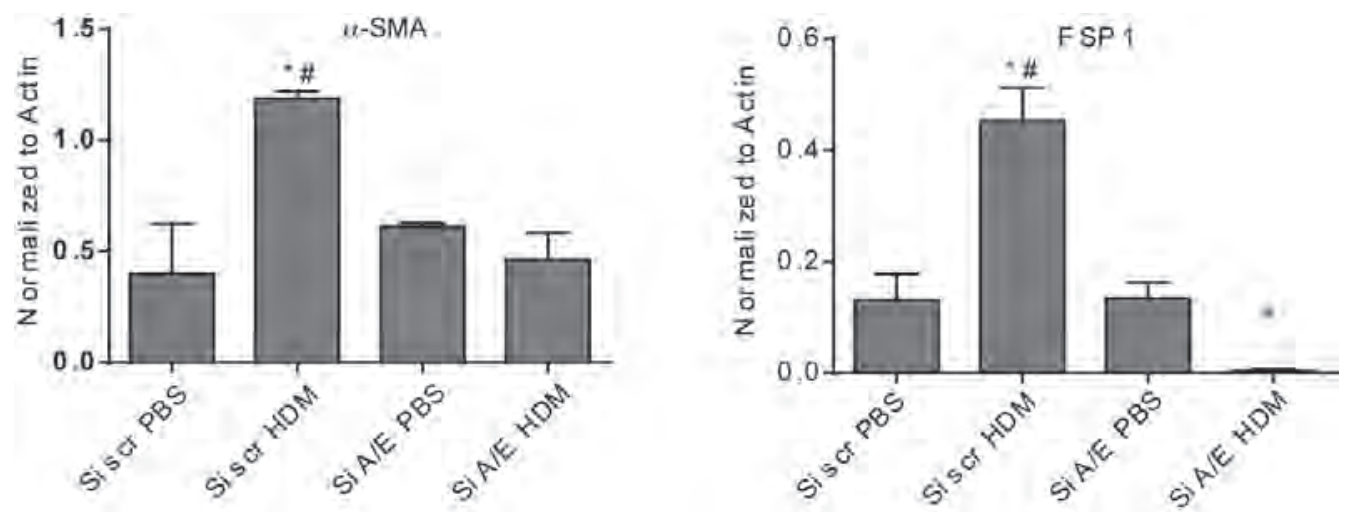

Supplementary Figure 4. Quantitaion of bands in the western blots in figure 6D by densitometry. All samples were normalized to actin.* indicates $\mathrm{p}<0.05$ as compared to PBS controls, \# indicates $\mathrm{p}<0.05$ as compared to their HDM samples by ANOVA.

\section{DISCUSSION}

Perturbations in ER homeostasis can cause ER stress, and when unresolved, ER stress is known to activate cell death [32]. Recent reports suggest that phosphorylation of ER stress transducer IRE1 (P-IRE) and subsequent $\mathrm{X}$-box binding protein 1 (XBP-1) activation is required to induce mucus metaplasia in the lungs of mice challenged with ovalbumin $[17,18]$. Those reports did not address the implications of ER stress in other facets of asthma, such as epithelial apoptosis, airway hyperresponsiveness and fibrosis. In the current investigation we sought to determine the mechanism by which HDM, a common aeroallergen, induces multiple facets of human asthma in mice. Our results demonstrate induction of severe ER stress in human nasal and bronchial epithelial cells, as well as in mice after administration of HDM. Furthermore, we also found that HDM-induced ER stress is associated with airway epithelial cell death, hyperresponsiveness and subsequent airway fibrosis in mice.

In contrast to recent reports $[17,18]$, our studies with human epithelial cells demonstrated that HDM-mediated activation of IRE1 is not consistent in primary nasal or bronchial epithelial cells. We observed increased phosphorylation of IRE1 in HDM challenged mice as compared to ovalbumin, LPS or ovalbumin/LPS challenged mice. However, we did not observe any downstream XBP-1 activation as in other published reports $[17,18]$. Activation of PKR-like ER kinase (PERK), phosphorylation of elF2 $\alpha$, or expression of ATF4, was also not observed. Instead, we showed robust activation of ATF6 $\alpha$ and caspase3 in human epithelial cells and believe that the differences in the activation of specific ER stress transducers may be due to the complex signaling pathways activated in the epithelium by HDM, 
compared to the antigen ovalbumin or TLR4 agonist, LPS $[5,11]$. Thus, our results indicate a multifaceted mechanism of allergen-specific activation of ER stress mediators in mouse and human airway epithelial cells.

HDM challenge of human primary nasal and bronchial epithelial cells, as well as HDM exposure of mice, demonstrated consistent robust activation of chaperones, such as GRP94, GRP78 (Bip), ATF6 ${ }^{50 k D a}$, a PDI-ERp57 and CHOP. Consistent with previously published data, our results demonstrate that chronic ER stress-mediated activation of ATF6 $\alpha$ led to increases in specific protein folding enzymes and subsequent activation of apoptotic executioner caspase-3 [14, 33, 34]. Interestingly, knockdown of ATF6 $\alpha$ in human bronchial epithelial cells, as well as ATF6 $\alpha$ and ERP57 in mice, resulted in decreased airway epithelial apoptosis as measured by activation of caspase-3. With the exception of GRP78 and CHOP [18], this up-regulation of specific ER stress and pro-apoptotic markers in allergic asthma has not yet been documented. It has been also suggested that chronic activation of ATF6 $\alpha$ can lead to the induction of $\mathrm{CHOP}$ and subsequent up regulation of proapototic Bak-mediated activation of caspases and apoptosis [16, 35-37]. Accordingly, our results suggest that ATF6 $\alpha$ activation leads to up regulation of CHOP as well as ERp57, which is capable of inducing disulfide (-S-S-) mediated oligomerization of Bak and induction of intrinsic apoptosis [16].

Our in vitro results in human epithelial cells provide a clue that ATF6 $\alpha$ as a transcription factor is responsible for ERp57 expression. As shown by others, ATF6 $\alpha$ is also known to regulate inflammatory responses in models of other diseases $[38,39]$ alluding to the possibility that ATF6 $\alpha$ could be regulating HDM-induced inflammatory responses in the lung.

As reported in neuronal diseases, a severe ER stress response can lead to neuronal cell death $[16,40]$. Recent studies on ER stress-mediated neuronal apoptosis have also shown involvement of ERp57 in disulfide-mediated oligomerization of proapoptotic Bak [16]. Our work supports the notion that ER stress-induced ERp57 mediates Bak oligomerization and apoptosis of lung epithelial cells during HDM challenge. Furthermore, our results also showed that ERp57 mediated oligomerization of Bak and apoptosis was associated with airway fibrosis. Based on these results, and our data demonstrating ATF6dependent induction of ERp57, it is reasonable to speculate that ERp57 could be regulating apoptosis of epithelial cells downstream of ATF6 during HDM challenge. However, the role of ATF6 $\alpha$ and ERp57 in regulating airway hyperresponsiveness is unknown at this point. Therefore, in the future it would be interesting to conduct careful experiments in mice with lung epithelial cell specific ablation of ERp57 and/or ATF6 $\alpha$ to determine the role of these two proteins in allergic airway diseases. 
ER stress transducers, such as ATF6 and CHOP, are known to play a prominent role in apoptosis of alveolar type II epithelial cells in fibrotic lung diseases, such as Idiopathic Pulmonary Fibrosis (IPF) [19, 20] and Hermansky Pudlak Syndrome (HPS) [21]. Recent studies have suggested that asthmatics and HDM based mouse models of asthma develop sub-epithelial thickening marked by aSMA (smooth muscle hyperplasia) and increased collagen deposition, [2-4] resulting in peribronchiolar fibrosis. Accordingly results presented here show that HDM induces severe ER stress, leading to apoptosis of airway epithelial cells and subsequent fibrosis.

Complex allergens, such as HDM [5], may induce a physiological state that requires an increase in protein synthesis and folding (e.g. production of high levels of mucin, cytokines and surfactants) and create an imbalance in synthesis and capacity to fold, which in turn may increase misfolded proteins in the ER, eliciting the ER stress response and ultimately, apoptosis [15]. Furthermore, the allergen dependent chronic activation of ER stress and apoptosis can cause repeated injury to the airway epithelium. In fact, injured epithelium in human asthmatics as well as in mouse models up regulate profibrotic growth factors, stimulating proliferation of the underlying smooth muscle cells, and subsequently leading to the deposition of extracellular matrix proteins [41]. In our studies, we were unable to observe the up-regulation of various pro-fibrotic growth factors, perhaps as a consequence of the protracted time of in vivo analyses.

Eosinophilic inflammation is one of the hallmarks of allergic asthma [42]. In our study, we found that knocking down ATF6 $\alpha$ and ERP57 resulted in partial attenuation of eosinophilic influx and lymphocytes in the BALF of HDM-challenged mice, indicating that ER stress may play a role in HDMinduced inflammatory responses. Our results also show that ER stress and apoptosis are associated with another critical facet of asthma, airway hyperresponsiveness to methacholine. HDM-induced tissue resistance $(G)$ and tissue stiffness $(H)$ were decreased in mice after knockdown of ATF6 $\alpha$ and ERp57. It is tempting to speculate that these changes in respiratory mechanics are due to altered permeability of the small airways in association with enhanced apoptosis of epithelial cells, which could perhaps allow increased access of methacholine to smooth muscle cells $[43,44]$. Interestingly we did not observe statistically significant differences in central airway resistance ( $\mathrm{Rn})$ in HDM-challenged, Si-scr mice as compared to HDM-challenged, Si-ERp57+ATF6 $\alpha$ mice. The mechanisms of uncoupling of $R_{n}$ in these models are yet to be determined.

Collectively, our work illuminates a previously unexplored mechanism for HDM-induced airway inflammation and fibrosis via ER stress and apoptosis of epithelial cells. We also show that knocking 
down ER stress transducers ATF6 $\alpha$ and ERP57 decreases HDM-induced apoptosis of airway epithelial cells, bronchiolar fibrosis, inflammation, and airway hyperresponsiveness. 


\section{REFERENCES}

1. Roche, W.R., et al., Subepithelial fibrosis in the bronchi of asthmatics. Lancet, 1989. 1(8637): $\mathrm{p}$. 520-4.

2. Noble, P.B., et al., Airway Smooth Muscle Dynamics and Hyperresponsiveness: In and outside the Clinic. J Allergy (Cairo), 2012. 2012: p. 157047.

3. James, A.L., et al., Airway smooth muscle hypertrophy and hyperplasia in asthma. Am J Respir Crit Care Med, 2012. 185(10): p. 1058-64.

4. Johnson, J.R., et al., Continuous exposure to house dust mite elicits chronic airway inflammation and structural remodeling. Am J Respir Crit Care Med, 2004. 169(3): p. 378-85.

5. Gregory, L.G. and C.M. Lloyd, Orchestrating house dust mite-associated allergy in the lung. Trends Immunol, 2011. 32(9): p. 402-11.

6. Nelson, R.P., Jr., et al., Allergen-specific IgE levels and mite allergen exposure in children with acute asthma first seen in an emergency department and in nonasthmatic control subjects. J Allergy Clin Immunol, 1996. 98(2): p. 258-63.

7. Chapman, M.D., et al., Nomenclature and structural biology of allergens. J Allergy Clin Immunol, 2007. 119(2): p. 414-20.

8. Fahlbusch, B., et al., The effect of storage on allergen and microbial agent levels in frozen house dust. Allergy, 2003. 58(2): p. 150-3.

9. Topp, R., et al., Repeated measurements of allergens and endotoxin in settled house dust over a time period of 6 years. Clin Exp Allergy, 2003. 33(12): p. 1659-66.

10. Da Silva, C.A., et al., Chitin particles are multifaceted immune adjuvants. Am J Respir Crit Care Med, 2010. 182(12): p. 1482-91.

11. Ryu, J.H., et al., Distinct TLR-mediated pathways regulate house dust mite-induced allergic disease in the upper and lower airways. J Allergy Clin Immunol, 2013. 131(2): p. 549-61.

12. Lan, R.S., G.A. Stewart, and P.J. Henry, Role of protease-activated receptors in airway function: $a$ target for therapeutic intervention? Pharmacol Ther, 2002. 95(3): p. 239-57.

13. Tabas, I. and D. Ron, Integrating the mechanisms of apoptosis induced by endoplasmic reticulum stress. Nat Cell Biol, 2011. 13(3): p. 184-90.

14. Hetz, C., The unfolded protein response: controlling cell fate decisions under ER stress and beyond. Nat Rev Mol Cell Biol, 2012. 13(2): p. 89-102.

15. Zhang, K. and R.J. Kaufman, From endoplasmic-reticulum stress to the inflammatory response. Nature, 2008. 454(7203): p. 455-62.

16. Hoffstrom, B.G., et al., Inhibitors of protein disulfide isomerase suppress apoptosis induced by misfolded proteins. Nat Chem Biol, 2010. 6(12): p. 900-6.

17. Schroeder, B.W., et al., AGR2 is induced in asthma and promotes allergen-induced mucin overproduction. Am J Respir Cell Mol Biol, 2012. 47(2): p. 178-85.

18. Martino, M.B., et al., The ER stress transducer IRE1beta is required for airway epithelial mucin production. Mucosal Immunol, 2013. 6(3): p. 639-54.

19. Korfei, M., et al., Epithelial endoplasmic reticulum stress and apoptosis in sporadic idiopathic pulmonary fibrosis. Am J Respir Crit Care Med, 2008. 178(8): p. 838-46.

20. Lawson, W.E., et al., Endoplasmic reticulum stress enhances fibrotic remodeling in the lungs. Proc Natl Acad Sci U S A, 2011. 108(26): p. 10562-7.

21. Mahavadi, P., et al., Epithelial stress and apoptosis underlie Hermansky-Pudlak syndromeassociated interstitial pneumonia. Am J Respir Crit Care Med, 2010. 182(2): p. 207-19.

22. Olson, N., et al., Nitric oxide and airway epithelial barrier function: regulation of tight junction proteins and epithelial permeability. Arch Biochem Biophys, 2009. 484(2): p. 205-13. 
23. Wu, R., Y.H. Zhao, and M.M. Chang, Growth and differentiation of conducting airway epithelial cells in culture. Eur Respir J, 1997. 10(10): p. 2398-403.

24. Jaspers, I., et al., Diesel exhaust enhances influenza virus infections in respiratory epithelial cells. Toxicol Sci, 2005. 85(2): p. 990-1002.

25. Anathy, V., et al., Redox amplification of apoptosis by caspase-dependent cleavage of glutaredoxin 1 and S-glutathionylation of Fas. J Cell Biol, 2009. 184(2): p. 241-52.

26. Reynaert, N.L., et al., Dynamic redox control of NF-kappaB through glutaredoxin-regulated Sglutathionylation of inhibitory kappaB kinase beta. Proc Natl Acad Sci U S A, 2006. 103(35): p. 13086-91.

27. Wilson, R.H., et al., Allergic sensitization through the airway primes Th17-dependent neutrophilia and airway hyperresponsiveness. Am J Respir Crit Care Med, 2009. 180(8): p. 720-30.

28. Riesenfeld, E.P., et al., Inhaled salmeterol and/or fluticasone alters structure/function in a murine model of allergic airways disease. Respir Res, 2010. 11: p. 22.

29. Pantano, C., et al., Nuclear factor-kappaB activation in airway epithelium induces inflammation and hyperresponsiveness. Am J Respir Crit Care Med, 2008. 177(9): p. 959-69.

30. Poynter, M.E., C.G. Irvin, and Y.M. Janssen-Heininger, Rapid activation of nuclear factor-kappaB in airway epithelium in a murine model of allergic airway inflammation. Am J Pathol, 2002. 160(4): p. 1325-34.

31. Ma, Y. and L.M. Hendershot, Delineation of a negative feedback regulatory loop that controls protein translation during endoplasmic reticulum stress. J Biol Chem, 2003. 278(37): p. 34864-73.

32. Wang, S. and R.J. Kaufman, The impact of the unfolded protein response on human disease. J Cell Biol, 2012. 197(7): p. 857-67.

33. Galindo, I., et al., The ATF6 branch of unfolded protein response and apoptosis are activated to promote African swine fever virus infection. Cell Death Dis, 2012. 3: p. e341.

34. Roberson, E.C., et al., Influenza induces endoplasmic reticulum stress, caspase-12-dependent apoptosis, and c-Jun $\mathrm{N}$-terminal kinase-mediated transforming growth factor-beta release in lung epithelial cells. Am J Respir Cell Mol Biol, 2012. 46(5): p. 573-81.

35. Yao, S., et al., Activating transcription factor 6 mediates oxidized LDL-induced cholesterol accumulation and apoptosis in macrophages by up-regulating CHOP expression. J Atheroscler Thromb, 2013. 20(1): p. 94-107.

36. Fu, H.Y., et al., Ablation of C/EBP homologous protein attenuates endoplasmic reticulummediated apoptosis and cardiac dysfunction induced by pressure overload. Circulation, 2010. 122(4): p. 361-9.

37. McCullough, K.D., et al., Gadd153 sensitizes cells to endoplasmic reticulum stress by downregulating Bcl2 and perturbing the cellular redox state. Mol Cell Biol, 2001. 21(4): p. 1249-59.

38. Cao, S.S., et al., The unfolded protein response and chemical chaperones reduce protein misfolding and colitis in mice. Gastroenterology, 2013. 144(5): p. 989-1000 e6.

39. Cao, S.S. and R.J. Kaufman, Unfolded protein response. Curr Biol, 2012. 22(16): p. R622-6.

40. Ono, Y., et al., A Sigma-1 Receptor Antagonist (NE-100) Prevents Tunicamycin-Induced Cell Death via GRP78 Induction in Hippocampal Cells. Biochem Biophys Res Commun, 2013.

41. Holgate, S.T., et al., The role of the airway epithelium and its interaction with environmental factors in asthma pathogenesis. Proc Am Thorac Soc, 2009. 6(8): p. 655-9.

42. Amelink, M., et al., Three phenotypes of adult-onset asthma. Allergy, 2013. 68(5): p. 674-80.

43. Bates, J.H., et al., The synergistic interactions of allergic lung inflammation and intratracheal cationic protein. Am J Respir Crit Care Med, 2008. 177(3): p. 261-8.

44. Poynter, M.E., et al., NF-kappa B activation in airways modulates allergic inflammation but not hyperresponsiveness. J Immunol, 2004. 173(11): p. 7003-9. 


\section{CHAPTER 3}

Epithelial NF- $\kappa B$ orchestrates house dust mite-induced airway inflammation, hyperresponsiveness, and fibrotic remodeling

Jane E. Tully, Sidra M. Hoffman, Karolyn G. Lahue, James D. Nolin, Vikas Anathy, Lennart K. Lundblad, Nirav Daphtary, Minara Aliyeva, Kendall E. Black, Anne E. Dixon, Matthew E. Poynter, Charles G. Irvin, and Yvonne M.W. Janssen-Heininger

Journal of Immunology 2013; 191. 5811-21 


\section{ABSTRACT}

NF-KB activation within the epithelium has been implicated in the pathogenesis of asthma, yet the exact role of epithelial NF-KB in allergen-induced inflammation and airway remodeling remains unclear. In the present study, we utilized an intranasal House Dust Mite (HDM) extract exposure regimen time course in BALB/c mice to evaluate inflammation, NF-kB activation, airway hyperresponsiveness (AHR), and airway remodeling. We utilized $\mathrm{CC} 10-\mathrm{I} \mathrm{KB} \alpha_{\mathrm{SR}}$ transgenic mice to evaluate the functional importance of epithelial NF-KB in response to HDM. After a single exposure of HDM, mRNA expression of pro-inflammatory mediators was significantly elevated in lung tissue of WT mice, in association with increases in nuclear RelA and RelB, components of the classical and alternative NF-KB pathway, respectively, in the bronchiolar epithelium. In contrast, $\mathrm{CC} 10-\mathrm{IKB}_{\mathrm{SR}}$ mice displayed marked decreases in nuclear RelA and RelB and mRNA expression of pro-inflammatory mediators compared to WT mice. After 15 challenges with HDM, WT mice exhibited increases in inflammation, airway hyperresponsiveness, mucus metaplasia and peri-bronchiolar fibrosis. $\mathrm{CC} 10-\mathrm{IKB}_{\mathrm{SR}}$ transgenic mice displayed marked decreases in neutrophilic infiltration, tissue damping, and elastance parameters, in association will less peribronchiolar fibrosis and decreases in nuclear RelB in lung tissue. However, central airway resistance and mucus metaplasia remained elevated in $\mathrm{CC} 10-\mathrm{IKB}_{\mathrm{SR}}$ transgenic mice, in association with continued presence of lymphocytes, and partial decreases in eosinophils and IL-13. The current study demonstrates that following airway exposure with an asthma-relevant allergen, activation of classical and alternative NF-KB pathways occur within the airway epithelium and may coordinately contribute to allergic inflammation, AHR and fibrotic airway remodeling. 


\section{INTRODUCTION}

The NF-KB pathway is a critical regulator of both innate and adaptive immune responses in a wide variety of cell types. Upon stimulation, the I kappa B kinase (IKK) signalsome, consisting of IKK $\beta$, IKK $\alpha$, and IKKY, is activated, leading to IKK $\beta$-mediated phosphorylation of IKB $\alpha$. Phosphorylation of IKB $\alpha$ in turn leads to its subsequent ubiquitination and degradation by the $26 \mathrm{~S}$ proteasome, thus allowing for transcription factor, RelA, to translocate to the nucleus. This event results in RelA-dependent transcription of genes important in cell survival, proliferation, and inflammation [1, 2]. A wide variety of agonists can activate the classical NF-KB pathway in lung epithelial cells and the resultant release of proinflammatory mediators crucial in the recruitment and activation of dendritic cells, lymphocytes, neutrophils, and many other cells in the lung [3]. Additionally, an alternative NF-KB pathway exists, which requires activation of NF-KB inducing kinase (NIK) and subsequent phosphorylation of IKK $\alpha$. IKK $\alpha$ in turn phosphorylates $\mathrm{p} 100$, leading to its partial processing to $\mathrm{p} 52$. This allows subsequent nuclear translocation of RelB/p52 and transcriptional activation of a subset of NF-KB dependent genes $[4,5]$. It was originally thought that the alternative NF-KB pathway played a predominant role in lymphocyte activation and lymphoid organ development [6]. However, recent work from our laboratory demonstrated that both classical and alternative NF-KB pathways are activated in lung epithelial cells in response to diverse pro-inflammatory stimuli and that both pathways coordinately regulate proinflammatory responses [7].

Activation of the classical NF-KB pathway within the airway epithelium has been demonstrated to play a critical role in acute inflammation and allergic airways disease. CC10-IKB $\alpha_{S R}$ transgenic mice, which are refractory to $1 \mathrm{~KB} \alpha$ degradation and NF-KB activation in the lung epithelium, were demonstrated to be strongly protected from airway inflammation induced by lipopolysaccharide [8]. Following intraperitoneal sensitization and challenge with ovalbumin (Ova), CC10-IKB $\alpha_{S R}$ transgenic mice showed a marked diminution of airway inflammation compared to WT littermate controls, although Ova-induced airways hyperresponsiveness (AHR) was unaffected in $C C 10-1 \kappa B \alpha_{S R}$ transgenic mice compared to controls [9]. A similar protection against Ova-induced allergic inflammation and peribronchiolar fibrosis has been observed in mice following epithelial-specific ablation of IKK $\beta$ [10].

It remains unclear to date whether activation of NF-KB within epithelial cells plays a role in the orchestration of inflammatory responses in vivo to an asthma-relevant allergen following sensitization via the airways. It also remains unknown whether both NF-KB pathways are activated following exposure to an antigen. House dust mite (HDM) is a multifaceted allergen to which up to $85 \%$ of 
asthmatics are allergic [11]. HDM has been shown to signal through the classical NF-KB pathway in human bronchial epithelial cells in vitro [12]. Therefore, the goal of the present study was to determine the activation of classical and alternative NF-KB in epithelial cells in vivo in response to HDM, and to address its effect on HDM-triggered airway inflammation, remodeling, mucus, and AHR. Our results demonstrate the functional importance of epithelial NF-KB in HDM-induced acute inflammatory responses, AHR, and airway remodeling. We also demonstrate activation of both classical and alternative NF-KB pathways in response to HDM. These findings illustrate the complexity of activation of the NF-KB pathways in settings of allergic airways disease and suggest a broader role for epithelial NF-KB in lung disease pathogenesis.

\section{MATERIALS AND METHODS}

Animal Studies. CC10-IKB $\alpha_{\mathrm{SR}}$ mice were generated as previously described [8] and backcrossed onto the $\mathrm{BALB} / \mathrm{CJ}(\mathrm{N}=10)$ (The Jackson Laboratories, Bar Harbor, ME) background. Transgene-negative littermates were used as a control. All experiments were approved by the University of Vermont Institutional Animal Care and Use Committee.

Cell Culture. A human bronchial epithelial cell line (HBE) was kindly provided by Dr. Albert van der Vliet and cultured as described previously $[13,14]$ and primary human nasal epithelial cells were cultured as

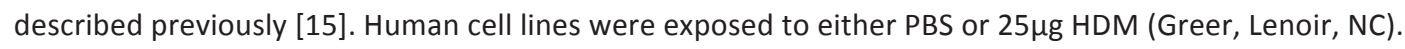
All protocols that utilize primary human nasal epithelial cells were approved by the Institutional Review Board.

HDM exposure. BALB/CJ mice (Jackson Laboratories, Bar Harbor, ME) were subjected to daily intranasal

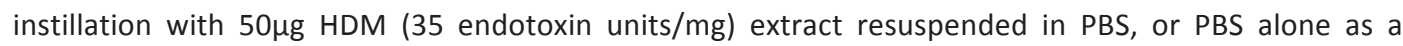
vehicle control. Briefly, mice were instilled with either 1 dose of HDM and euthanized: $2 \mathrm{~h}, 6 \mathrm{~h}$, or $24 \mathrm{~h}$ later or instilled with 3 doses of HDM on 3 consecutive days and euthanized $24 \mathrm{~h}$ thereafter. In addition, mice were exposed to HDM 5 days/week for 1, 2 or 3 weeks, (5, 10 or 15 instillations, respectively), and harvested $72 \mathrm{~h}$ after the final exposure (Fig. 1A).

Assessment of AHR. Mice subjected to 5, 10, or 15 administrations of HDM were anesthetized with an intraperitoneal injection of pentobarbital sodium $(90 \mathrm{mg} / \mathrm{kg})$, tracheotomised, and mechanically ventilated at 200 breaths/minute and assessed in response to increasing doses of methacholine (0, $3.125 \mathrm{mg}, 12.5 \mathrm{mg}$, and $25 \mathrm{mg}$ ). Respiratory mechanics were assessed with a forced oscillation technique on a computer controlled small animal ventilator (Flexi-vent ${ }^{\mathrm{TM}}, \mathrm{SCIREQ}, \mathrm{QC}$, Canada), as previously 
described $[16,17]$, and the parameters Newtonian resistance $(R n)$, tissue damping $(G)$, and elastance $(H)$ were calculated.

Serum IgG1 and IgE. Following euthanization, blood was collected by heart puncture and immediately spun through a microtainer and serum was separated. Analysis of serum IgG1 and IgE was performed via enzyme-linked immunosorbent assay (ELISA) methods, using $1 \mu \mathrm{g} / \mathrm{ml}$ HDM to coat the 96 well plates.

Bronchoalveolar lavage. Following euthanization, bronchoalveolar lavage (BAL) was collected using $1 \mathrm{ml}$ of PBS. Cell counts were determined (Advia 120 automated hematology analyser), and differential cells were analysed by the Hema3 kit (Fisher Scientific, Kalamazoo, MI) by counting a minimum of 300 cells per mouse as previously described [17].

$m R N A /$ protein analysis. Right lung lobes were flash frozen and pulverized for protein and mRNA. Total mRNA was isolated using the RNeasy kit (Qiagen, Valencia, CA). $1 \mu \mathrm{g}$ of mRNA was used to generate CDNA, followed by quantitative polymerase chain reaction (qPCR) using SYBR green (BioRad, Hercules, CA) via in order to assess expression of: Muc5ac, KC, MIP-2, GM-CSF, IL-6, CCL20, and IL-33. Primers were: KC (Fwd:5'-GCTGGATTCACCTCAAGAA-3', Rv:5'-TGGGGACACCTTTTAGCATC-3'), IL-6 (Fwd:5'CtGATGCTGGTGACAACCAC-3', Rv:5'-CAGAATtGCCATTGCACAAC-3'), CCL20 (Fwd:5'AAgACAGATGGCCGATGAAG-3', Rv:5'-AgCCCTTtTCACCCAGTTCT-3'), Muc5ac (Fwd:5'CAGTGAATTCTGGAGGCCAACAAGGTAGAG-3', Rv:5'-AGCTAAGCTTAGATCTGGTTGGGACAGCAGC-3'), MIP2 (Fwd:5'-AGTGAACTGCGCTGTCAATG-3', Rv:5'-TTCAgGgTCAAGGCAAACTT-3'), GM-CSF (Fwd:5'GGCCTTGgAAGCATGTAGAGG-3', Rv:5'- GgAGAACTCGTTAGAGACGACTT-3'), and IL-33 (Fwd:5'GCTGCGTCTGTTGACACATT-3', Rv:5'-GACTTGCAGGACAGGGAGAC-3'). The expression of all genes was normalized to the housekeeping gene, cyclophilin, (Fwd:5'- TTCCTCCTTTCACAGAATTATTCCA-3', Rv:5'CCGCCAGTGCCATTATGG-3') and calculated via the ddCt method. Antibodies for Western blot analysis were: RelB and NIK (Santa Cruz Biotchnologies, Santa Cruz, CA), Histone H3, p52, and phospho RelA536 (Cell Signaling Technology, Danvers, MA) and $\beta$-Actin (Sigma-Aldrich, St. Louis, MO). Enzyme-linked Immunosorbent assays (ELISA) were performed according to manufacturer's instructions ( $R$ \& $D$ Systems, Minneapolis, MN) on either BAL or homogenized lung extracts. Protein was equalized before analysis.

Histopathology/immunofluorescence/aSMA immunohistochemistry. Following euthanization, the left lobe was inflated with $4 \%$ paraformaldehyde and mounted in paraffin embedded $5 \mu \mathrm{m}$ sections. Hematoxylin and Eosin (H\&E) and Periodic Acid Schiff (PAS) imaging were all performed with 3 small bronchioles (x20 magnification) per animal. Mucus metaplasia was assessed with a blinded scoring system by two independent investigators with the following scale: 0 , no reactivity; 1 , minimal staining; 
2, moderate staining; and 3, prominent staining. Scores were averaged according to treatment group. Immunofluorescence was performed as previously described to detect nuclear RelA and RelB in situ [18]. RelA and RelB antibodies were purchased from Santa Cruz Biotechnology (Santa Cruz, CA). Staining for $\alpha$-SMA was performed on lung sections following incubation of slides for 20 minutes in $0.01 \mathrm{M}$ sodium citrate, $\mathrm{pH} 6.0$ at $95{ }^{\circ} \mathrm{C}$. Slides were then blocked with $2 \%$ normal goat serum for 30 minutes, incubated with monoclonal mouse antibody against $\alpha$-SMA (1:5000 dilution; Sigma Aldrich, St. Louis, MO) overnight at $4 \stackrel{\circ}{ } \mathrm{C}$, and then incubated in biotinylated anti-mouse $\lg$ for 30 minutes at room temperature. Subsequently, the slides were incubated in avidin-biotin- complex-alkaline phosphatase (Vectastain ABC-AP, Vector Laboratories, Burlingame, CA) for another 30 minutes at room temperature. After rinsing the sections in PBS, the substrate, Vector Red (Vector Laboratories), was added for 20 minutes, which reacts with the bound alkaline phosphatase, thus producing an intense red color. The slides were then counterstained in hematoxylin and imaged (x20 magnification) for analysis.

Assessment of collagen content. Collagen was assessed from the upper right lobe of the lung after an overnight digestion with $10 \mathrm{mg}$ pepsin in $0.5 \mathrm{M}$ acetic acid. Quantification was performed by the Sircol Assay according to manufacturer's instructions (Accurate Chemical and Scientific corp, Westbury, NY). Masson's Trichrome reactivity was evaluated in 3 bronchioles (x20 magnification) per animal, and analyzed by two blinded investigators with the following scale: 0 , no reactivity; 1 , minimal staining; 2 , moderate staining; and 3, prominent staining.

Statistical Analysis. All data were evaluated using Graphpad Prism 6 Software (Graphpad, Inc, San Diego, CA). A one-way ANOVA was used with Bonferoni corrections to adjust for multiple comparisons and all p-values $<0.05$ were considered statistically significant. Histopathological/aSMA IHC scores were analyzed using the Kruskal-Wallis test and Dunn's multiple comparison post hoc tests.

\section{RESULTS}

Inflammatory response in BALB/c mice exposed to HDM. Because of the well-known role of NF-KB in the orchestration of inflammation, we first sought to assess the extent and kinetics of the HDM-induced inflammatory response through a time-course analysis illustrated schematically in Fig. 1A. Neutrophils in bronchoalveolar lavage fluid (BAL) were significantly increased $6 \mathrm{~h}$ following a single intranasal challenge with HDM, and remained elevated until $24 \mathrm{~h}$ post 3 challenges, as compared to PBS controls (Fig. 1B). It is important to note that neutrophils were elevated $24 \mathrm{~h}$ following exposure to the vehicle PBS, potentially indicative of a non-specific response to the intranasal instillation. Neutrophils were also 
detectable in BAL $72 \mathrm{~h}$ post 5, 10, or 15 challenges, with statistically significant increases occurring in response to HDM $72 \mathrm{~h}$ post 15 challenges. Although macrophages in BAL tended to increase in response to HDM, these trends were not statistically significant. No eosinophils or lymphocytes were detected in BAL up to 24 h post 3 challenges. In contrast, robust increases in eosinophils and lymphocytes occurred in BAL $72 \mathrm{~h}$ post 10 and 15 challenges with HDM compared to PBS controls (Fig. 1B). Although some fluctuations in the total number of macrophages in BAL were observed throughout these time points, these were not statistically significant (Fig. 1B)
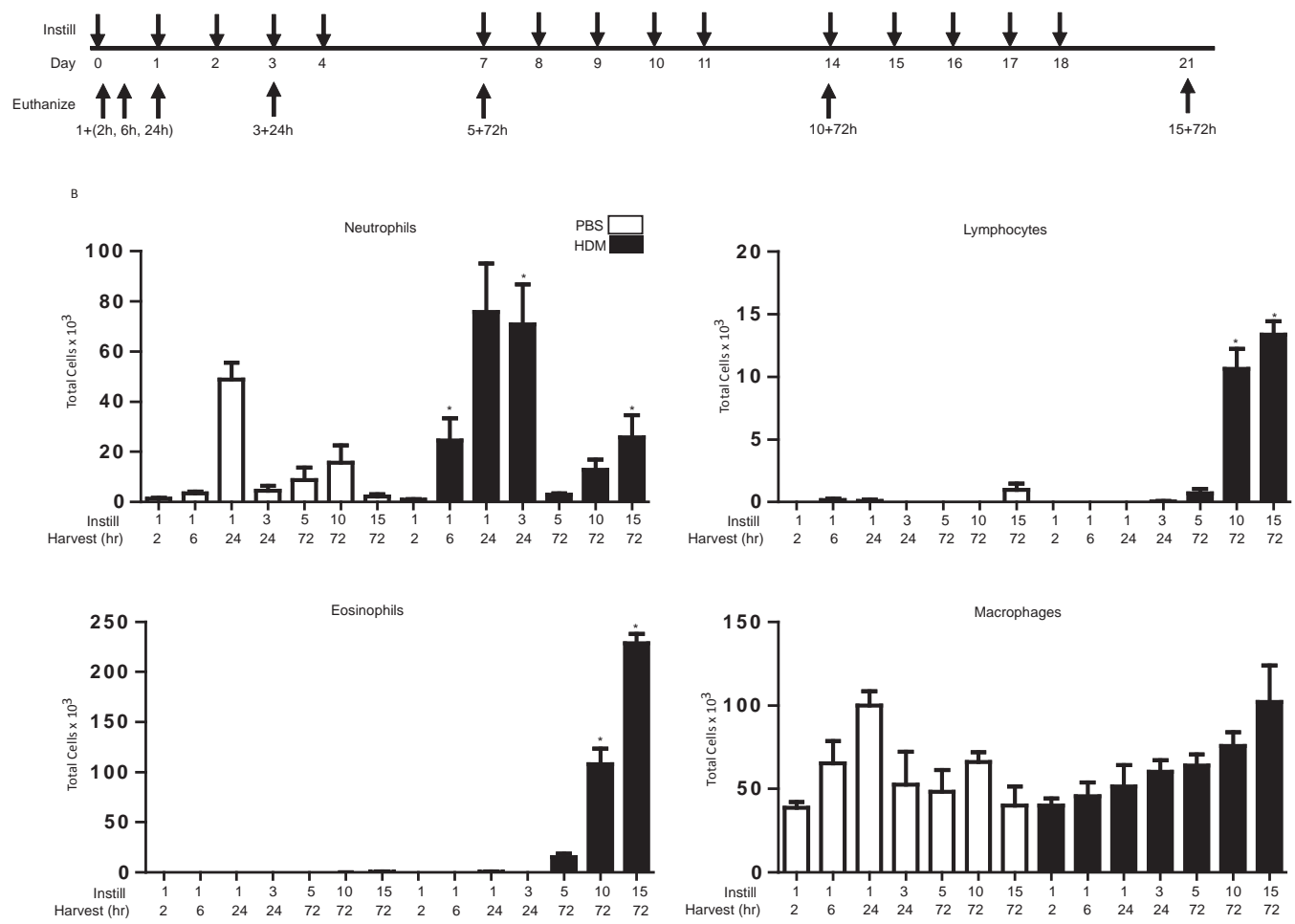

Figure 1. Analysis of cell totals and differentials in BAL of mice exposed to HDM. (A) Schematic depicting the

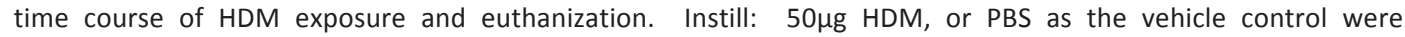
administered intranasally once at the days indicated via downward arrows. Mice were euthanized $2 \mathrm{~h}, 6 \mathrm{~h}$ or $24 \mathrm{~h}$ post a single challenge, $24 \mathrm{~h}$ after 3 challenges or $72 \mathrm{~h}$ after 5,10 , or 15 challenges, indicated by the upward arrows. (B) Mice ( $n=5 /$ group/time point) were exposed to PBS or HDM according to the schematic in A, and BAL was assessed for neutrophils, eosinophils, lymphocytes, and macrophages. ${ }^{*} p<0.05$ (ANOVA) compared to the PBS group at the same time point.

Increases in nuclear localization of RelA and RelB in airway epithelium in response to HDM. We next evaluated a potential role for epithelial NF-KB in response to HDM. We investigated kinetics of 
activation of both classical and alternative pathways in the bronchiolar epithelium via the assessment of nuclear presence of RelA and RelB. Nuclear presence of RelA and RelB (indicated by yellow staining) within the bronchiolar epithelium was increased 2 and $6 \mathrm{~h}$ following a single challenge with HDM, compared to PBS controls in the nuclei (Fig. 2). Nuclear RelA decreased to control reactivity by 24 h, but increased again $24 \mathrm{~h}$ post- 3 challenges with HDM, indicative of a bi-modal activation pattern (Fig. 2). In contrast, increases in nuclear RelB were apparent throughout the time course evaluated here (Fig. 2). No clear evidence for nuclear localization of RelA or RelB within the airway epithelium was apparent 72 h after 5, 10 or 15 challenges with HDM compared to PBS (data not shown).
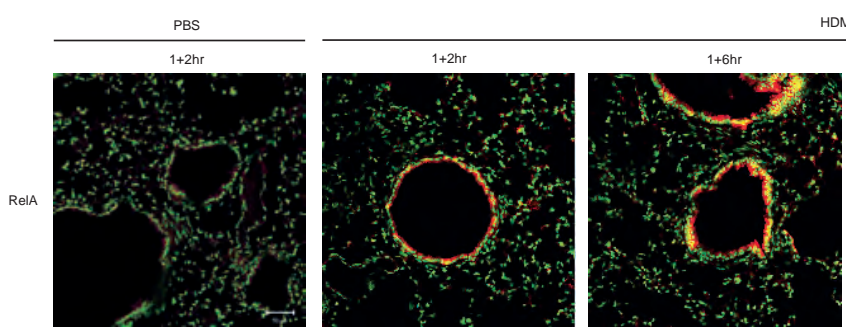

HDM
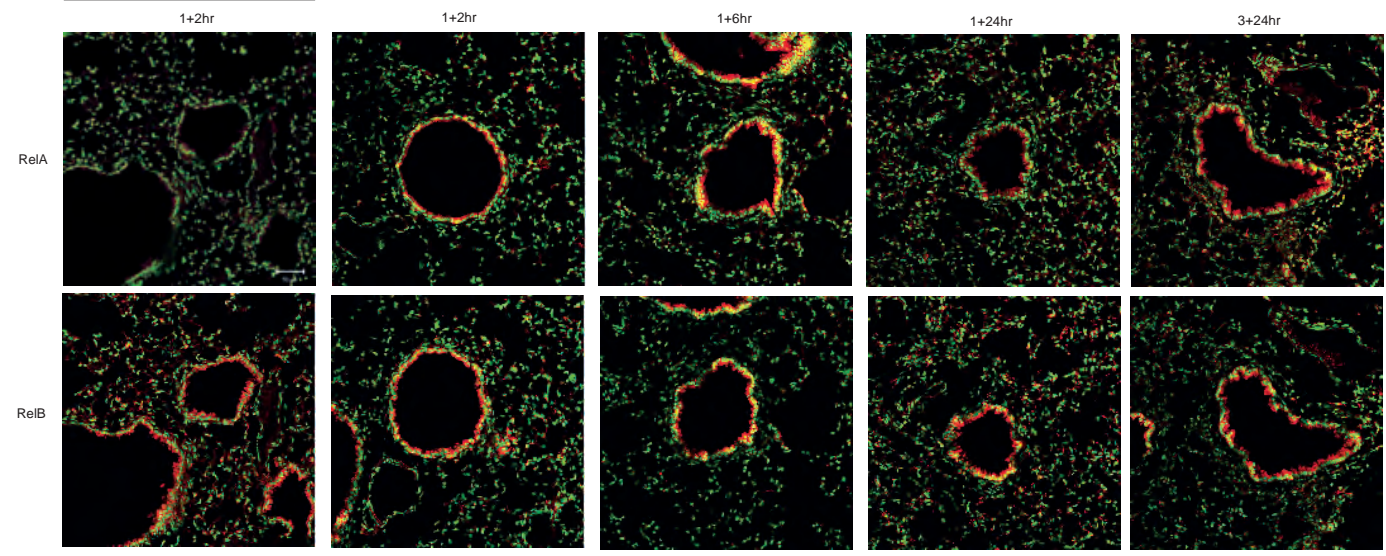

Figure 2. Assessment of nuclear localization of RelA and RelB in airway epithelium in response to HDM. BALB/C mice were exposed to PBS and HDM and harvested as indicated. De-paraffinized lung sections were incubated with antibodies directed against RelA or RelB, followed by fluorophore-conjugated secondary antibodies, and the nuclei were counterstained with Sytox Green. Images were captured by laser scanning confocal microscopy using identical instrument settings in all groups. Images are representative results of two independent experiments, $\mathrm{n}=5$ /group/time. RelA/RelB: red, DNA: green, Nuclear RelA/RelB: yellow. Scale bar=50 $\mu \mathrm{m}$.

Pro-inflammatory mediator expression following acute HDM exposures. In order to investigate the early inflammatory response after HDM exposure, we evaluated mRNA expression of Interleukin-33 (IL-33), Keratinocyte Derived Chemokine (KC), Granulocyte Macrophage Colony Stimulating-Factor (GM-CSF), Macrophage Inflammatory Protein-2 ( MIP-2), Chemokine (C-C motif) ligand 20 (CCL20), Interleukin-6 (IL-6), Interleukin-25 (IL-25) and Thymic Stromal Lymphopoietin (TSLP), pro-inflammatory mediators shown to be produced by epithelial cells. Expression levels of all genes analyzed were significantly increased following $2 \mathrm{~h}$ exposure HDM, with the exception of IL-25 and TSLP, whose expression did not change (data not shown). mRNA expression of CCL20, GM-CSF, MIP-2, KC, and IL-6 remained elevated 6 
h after a single administration of HDM, and tended to decrease towards control levels thereafter (Fig S1).
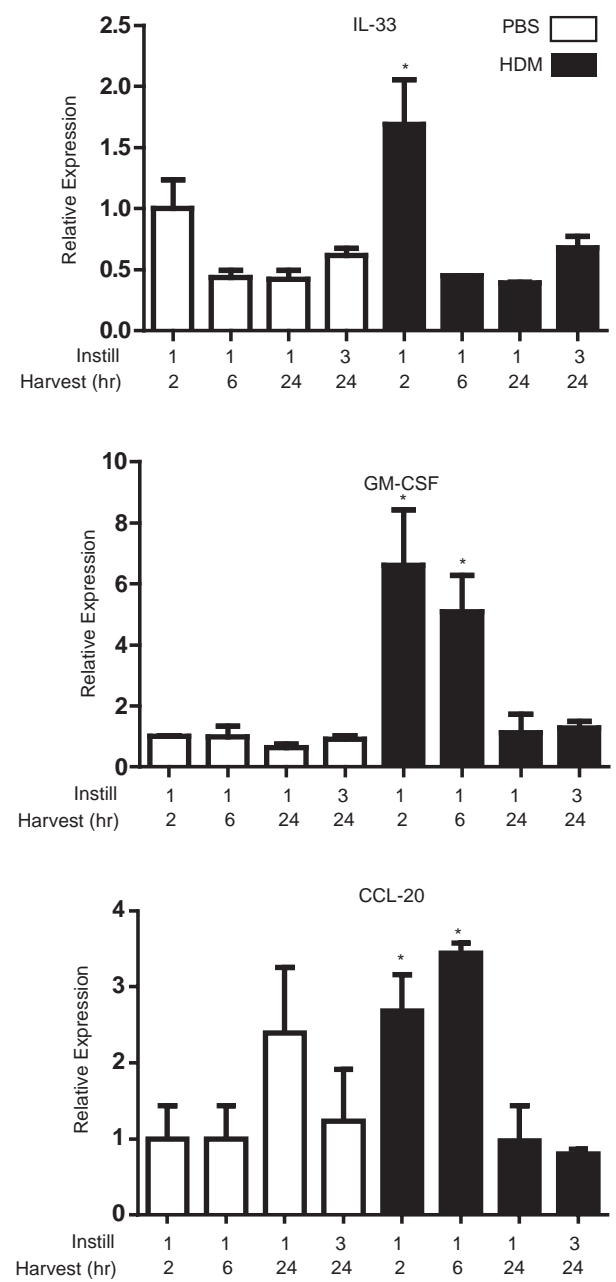
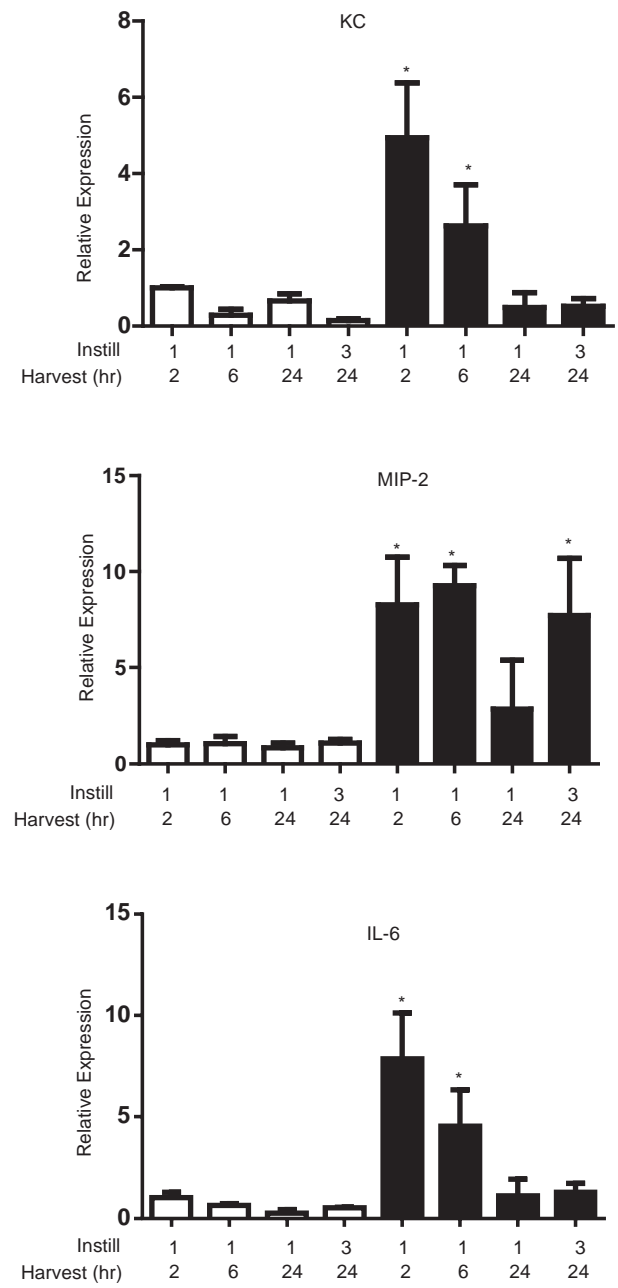

Supplementary Figure 1. Pro-inflammatory mediator mRNA expression analysis following HDM exposure. $\mathrm{BALB} / \mathrm{c}$ mice were exposed to a single dose of either PBS or HDM and harvested 2, 6, or $24 \mathrm{~h}$ following challenge. Additionally, mice were challenged for three consecutive days and harvested $24 \mathrm{~h}$ following the $3^{\text {rd }}$ challenge. mRNA isolated from homogenized lung tissue was analyzed for expression of IL-33, GM-CSF, CCL20, KC, MIP-2, and IL-6. Data represent 5 mice/group/time point. ${ }^{*} p<0.05$ (ANOVA) compared to the PBS group at the same time point.

Role of epithelial NF-KB in pro-inflammatory cytokine expression induced by HDM. We next sought to address the role of epithelial NF-KB in HDM-induced pro-inflammatory responses utilizing CC10-IKB $\alpha_{S R}$ transgenic mice. While nuclear RelA and RelB content increased in the bronchiolar epithelium in WT 
mice in response to $\mathrm{HDM}$, these increases were not observed in CC10-IKB $\alpha_{S R}$ mice exposed to HDM (Fig 3A). HDM- mediated increases in mRNA expression of CCL20, GM-CSF, MIP-2, KC, IL-33, and IL-6 in WT mice were strongly attenuated in $\mathrm{CC} 10-\mathrm{IKB}_{\mathrm{SR}}$ mice (Fig. $3 \mathrm{~B}$ ), demonstrating the importance of NF-KB activation in the bronchiolar epithelium in the orchestration of the acute pro-inflammatory responses to HDM.
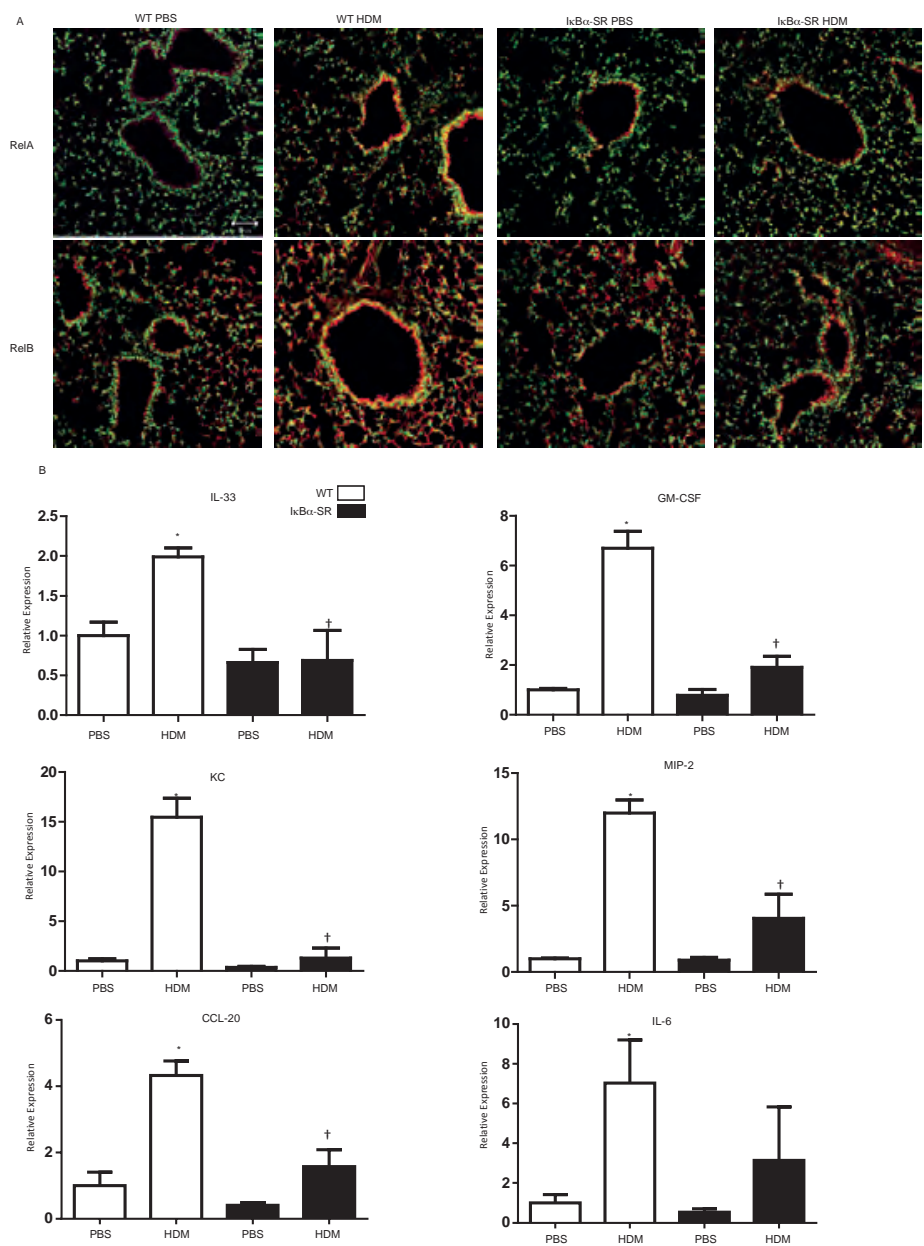

Figure 3. Nuclear RelA and RelB, and pro-inflammatory cytokine mRNA expression in CC10-NF-KB SR $_{S \text { mice }}$ following exposure to HDM. CC10-NF-KB $B_{S R}$ mice, or WT littermate controls were exposed to PBS or HDM for $2 \mathrm{~h}$. (A) Nuclear localization of RelA and RelB was assessed in lung tissues as described in Fig. 2. Images are representative results of two independent experiments with 5 mice/group, RelA/RelB, red; DNA, green; Nuclear RelA/RelB, yellow, scale bar=50 $\mu$ m. (B) Assessment of mRNA expression for IL-33, GM-CSF, CCL20, KC, MIP-2, and IL-6 in homogenized lung tissue. Data were normalized to cyclophilin and are presented as relative expression. Values reflect 6 mice/group/time point. ${ }^{*} p<0.05$ (ANOVA) compared to the PBS group at the same time point. $t p<0.05$ (ANOVA) compared to WT mice exposed to HDM. 
Effects of repeated HDM exposure on AHR, mucus metaplasia, and remodeling. We next subjected mice to 5,10 , and 15 challenges of HDM. Serum content of HDM-specific IgG1 and IgE showed no apparent increases $72 \mathrm{~h}$ following 5 days challenge. Marked increases in HDM-specific IgG1 and IgE were apparent following 10 and 15 challenges with HDM, compared to PBS controls, indicative of activation of adaptive immune responses (Fig. S2A). AHR, a feature of allergic airways disease, was assessed via forced oscillation mechanics using ascending doses of methacholine. Newtonian resistance (Rn) increased significantly over PBS controls after 10 and 15 challenges with HDM, while no changes were apparent in mice subjected to 5 challenges with HDM (Fig S2B). Tissue damping (G), which is indicative of tissue resistance and small airway dysfunction, was increased following 5 challenges HDM, and remained increased throughout 10 and 15 challenges. Elastance $(H)$ was also increased following 5, 10, and 15 challenges of HDM. Increases in elastance were most prominent after 10 challenges of HDM and tended to decrease after 15 challenges (Fig. S2B). Histo-pathological evaluation revealed a robust inflammatory response to HDM, with prominent peri-bronchiolar and perivascular cellular infiltrates being apparent following 10 and 15 challenges with HDM (Fig. S2C). Additionally, mucus metaplasia was apparent following 5, 10, and 15 challenges HDM, based upon staining with Periodic Acid Shiff reagent and Muc5ac expression (Fig. S2C and S2D). Another hallmark of severe allergic airways disease is peribronchiolar fibrotic remodeling. Following 15 challenges with HDM, overall collagen content increased in the lung tissue (Fig S2E), consistent with increases in peri-bronchiolar collagen deposition evaluated via Masson's Trichrome staining (Fig S2C). These results collectively demonstrate that the HDM exposure regimen used herein induces a number of the hallmark features of allergic airway disease. 

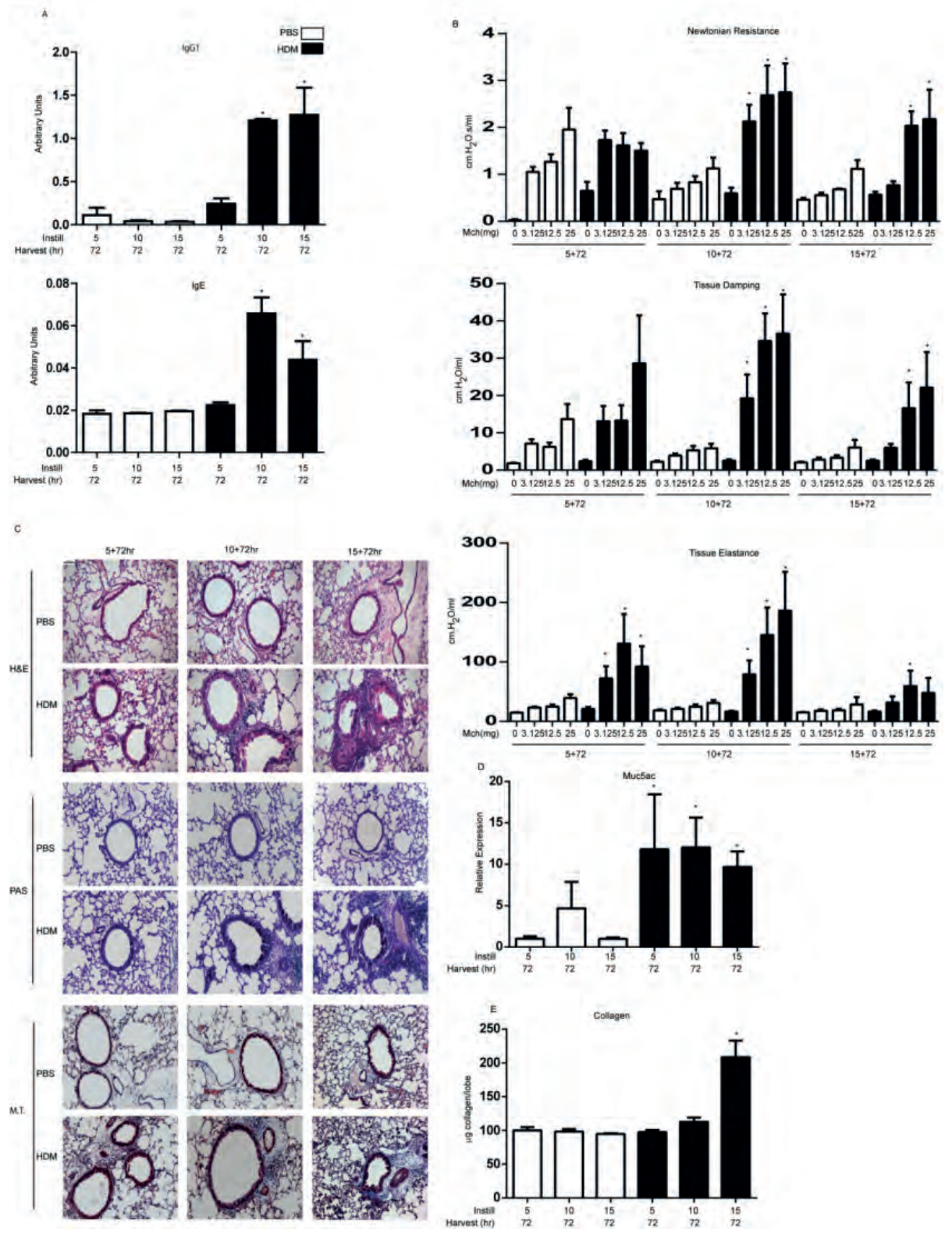

Supplementary Figure 2. Effects of Repeated HDM Exposure on HDM-specific IgG1 and IgE, AHR and airways remodeling. $\mathrm{BALB} / \mathrm{c}$ mice were exposed to HDM and harvested at the indicated time points. (A) Assessment of total IgG1 and IgE in serum. (B) Assessment of airway hyperresponsiveness in response to increasing doses $(3.125,12.5$, and $25 \mathrm{mg})$ of methacholine via forced oscillation mechanics. Parameters measured were Newtonian Resistance (Rn), Tissue Damping (G) and Tissue Elastance (H). (C) Histopathological analysis of inflammation (H\&E), mucus metaplasia (PAS) and peri-bronchiolar collagen deposition (M.T.) in mice exposed to HDM for the indicated times. Scale bar=50 $\mu \mathrm{m}$. (D) Analysis of Muc5ac mRNA expression in homogenized lung tissue. Data were normalized to cyclophilin and are presented as relative expression. (E) Quantification of 
collagen from the upper right lobe. Data represent 5 mice/group/time point. ${ }^{*} p<0.05$ (ANOVA) compared to the PBS group at the same time point.

Role of epithelial NF-KB in HDM-induced inflammation. We next determined the role of epithelial NF-KB on the inflammatory response induced following 15 challenges of HDM. HDM-mediated increases in total cell counts in BAL observed in WT mice were significantly decreased in CC10-IKBa $\alpha_{S R}$ mice (Fig. 4A). BAL eosinophils were significantly decreased in $\mathrm{CC} 10-\mathrm{I} K B \alpha_{S R}$ transgenic mice exposed to $\mathrm{HDM}$, while $\mathrm{BAL}$ lymphocytes remained elevated in $\mathrm{HDM}$ exposed $\mathrm{CC} 10-\mathrm{IKB}_{\mathrm{SR}}$ transgenic mice compared to WT littermates (Fig. 4B). Total macrophage number in BAL remained unchanged in CC10-IKB $\alpha_{S R}$ mice (Fig. 4B). In contrast HDM-mediated increases in BAL neutrophils in WT mice were completely attenuated in $\mathrm{CC} 10-\mathrm{IKB}_{\mathrm{SR}}$ mice. Consistent with these observations, increases in BAL content of the neutrophil chemoattractant, KC, in WT mice exposed to HDM were absent in CC10-IKB $\alpha_{S R}$ mice (Fig. 4C). HDMspecific IgG1 and IgE levels in serum were decreased slightly in CC10-IKB $\alpha_{S R} H D M$-exposed mice, although not significantly, compared to littermate controls, but remained elevated over PBS controls (Fig. 4D). Levels of IFN- $\gamma$, IL-13, and IL-17A in homogenized lung tissue were significantly decreased in CC10-NF-KB $B_{S R}$ mice exposed to HDM, compared to WT animals (Fig. 4E), collectively suggesting an attenuation of Th-mediated responses to HDM following epithelial-specific inhibition of NF-KB. We next sought to assess inhibition of nuclear RelA and RelB in lung tissue in CC10-NF-KB $\mathrm{SR}_{\mathrm{R}}$ mice exposed to HDM. After 15 challenges with HDM, no consistent increases in nuclear RelA or RelB were detected in the bronchiolar epithelium (data not shown), in contrast to earlier time points at which increases in nuclear RelA and RelB were readily observed (Fig 3). Assessment of homogenized lung tissue also showed variable and inconsistent patterns of nuclear RelA (data not shown). However, clear increases in nuclear RelB content were observed in WT mice, which were absent in the $\mathrm{CC} 10-\mathrm{IKB} \alpha_{\mathrm{SR}}$ transgenics (Fig. $4 \mathrm{~F})$. 

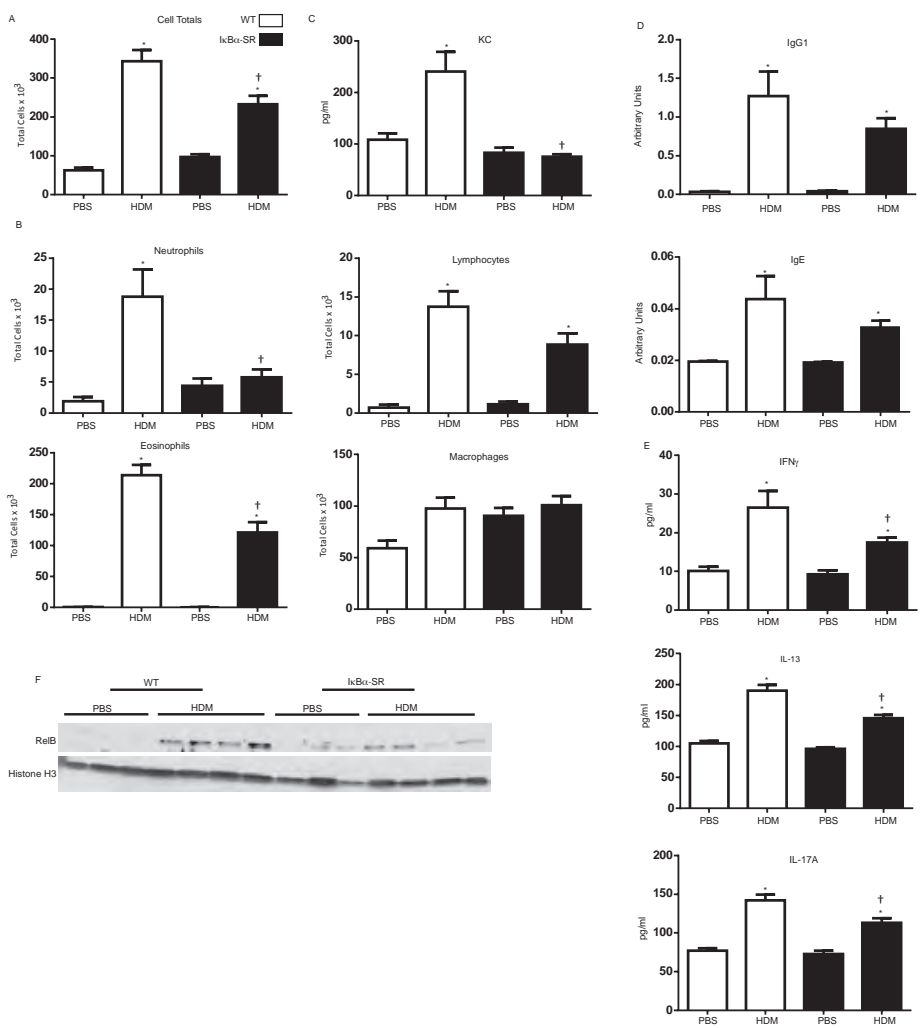

Figure 4. Assessment of HDM-induced inflamation, immunoglobins, T-cell cytokines, and nuclear RelB in WT or CC10-NF-KB $B_{S R}$ mice following 15 challenges with HDM. Assessment of (A) total cells and differential cell counts (B) and KC levels(C) in BAL. (D) Total IgG1 and IgE in serum. (E) IFNY, IL-13, and IL-17A in homogenized tissue by ELISA. (F) Nuclear content of RelB in homogenized lung tissue. Histone $\mathrm{H} 3$ is shown as a loading control. Data represents 8 mice/group/time point. ${ }^{*} p<0.05$ (ANOVA) compared to the PBS group at the same time point. ${ }^{\dagger} p<0.05$ (ANOVA) compared to WT mice exposed to HDM.

Role of epithelial NF-KB in HDM-induced AHR and remodeling. WT and CC10-IKBa $\alpha_{S R}$ mice subjected to 15 challenges with HDM demonstrated equivalent increases in Newtonian resistance (Rn). In contrast, HDM-mediated increases in tissue damping $(G)$ and elastance $(H)$ were significantly decreased in CC10$I_{\mathrm{KB}} \alpha_{\mathrm{SR}}$ mice compared to littermate controls (Fig. 5A). Consistent with attenuated inflammatory cell profiles in BAL, peri-bronchiolar infiltrates were attenuated CC10-IKB $\alpha_{S R}$ mice compared to controls (Fig. 5B). In contrast, mucus metaplasia was not observed to be decreased in HDM-exposed CC10-IKB $\alpha_{S R}$ mice, compared to WT animals (Fig. 5B+D). These findings are consistent with a lack of attenuation of HDM-induced Muc5AC mRNA (Fig. 5C) and modest decreases in IL-13 content (Fig. 4E). Biochemical analysis of collagen demonstrated that HDM-mediated increases in WT mice were significantly 
decreased in $\mathrm{CC} 10-\mathrm{IKB}_{\mathrm{SR}}$ transgenic mice (Fig.5E), consistent with less peri-bronchiolar collagen detected via histopathology (Fig. 5B+F). Furthermore, $\alpha$-Smooth Muscle Actin ( $\alpha$-SMA), a known marker of airway thickening and remodeling, was assessed via immunohistochemistry. Peri-bronchiolar $\alpha$-SMA staining was significantly increased following HDM administration in WT mice, as compared to PBS controls. In contrast, no increases in peri-bronchiolar $\alpha$-SMA reactivity were detected in CC10-IKB $\alpha_{S R}$ mice exposed to HDM, in comparison to PBS controls (Fig. $5 \mathrm{G}+\mathrm{H}$ ).
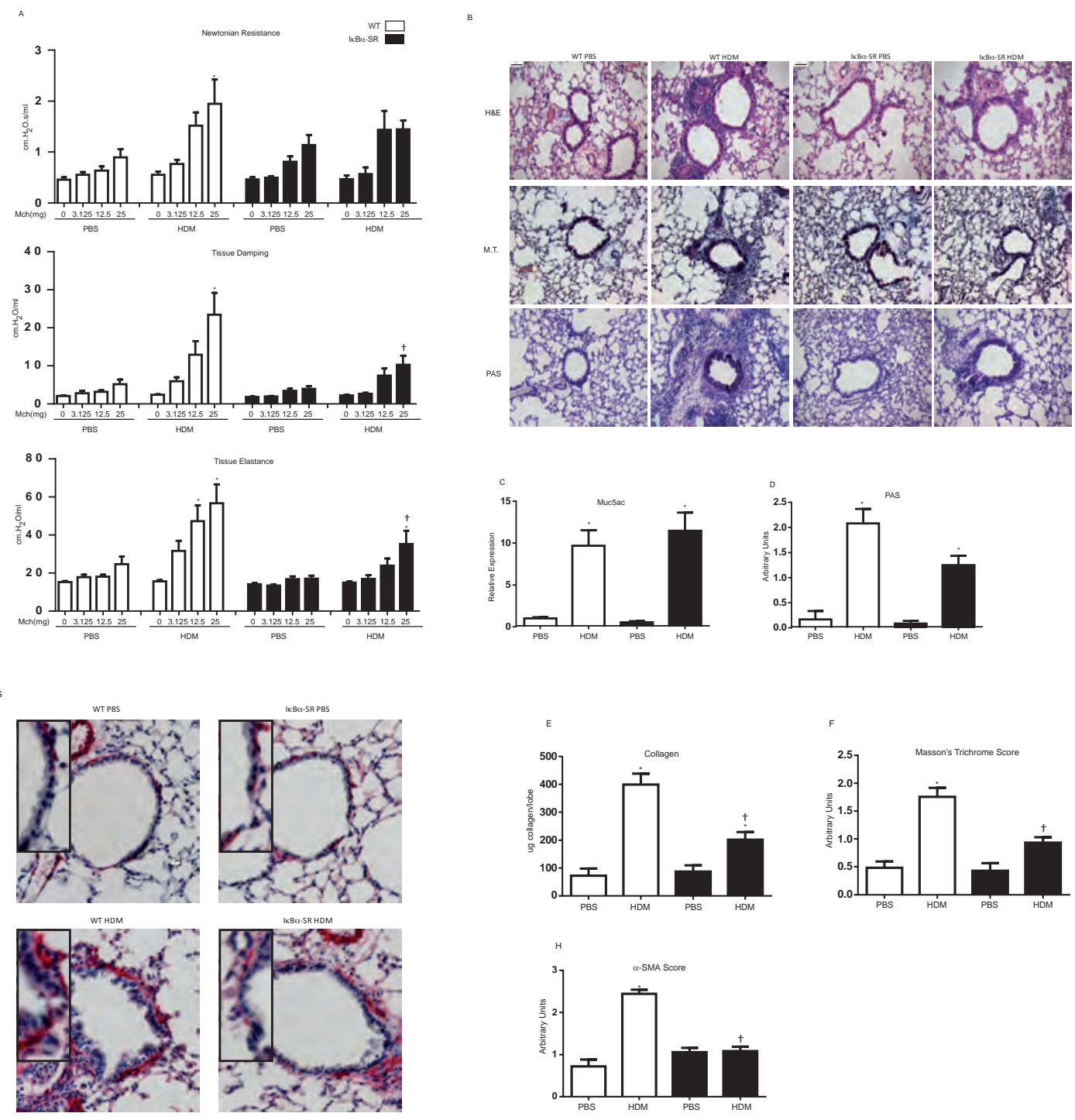
Figure 5. Assessment of HDM-induced alterations in respiratory mechanics and airway remodeling in WT or CC10-NF-KB $\mathrm{SR}_{\mathrm{SR}}$ mice following 15 challenges with HDM. (A) Evaluation of airway hyperresponsiveness via forced oscillation mechanics in response to 3.125, 12.5, and $25 \mathrm{mg}$ methacholine. Shown are the parameters Newtonian resistance (Rn), Tissue damping (G) and Elastance (H) assessed via forced oscillation (B) Histopathological evaluation of tissue inflammation (H\&E), mucus metaplasia (PAS) and peri-bronchiolar collagen deposition (M.T.). Scale bar $=50 \mu m$. (C) Analysis of Muc5ac mRNA expression in homogenized lung tissue. Data were normalized to cyclophilin and are presented as relative expression. (D) Quantification of mucus metaplasia. Bronchioles of similar size $(n=3)$ were analyzed/mouse by two blinded scorers, and average scores presented as average units. $(E)$ Assessment of collagen from the upper right lobe. (F) Quantification of peri-bronchiolar collagen deposition. Bronchioles of similar size $(n=3)$ were analyzed/mouse by two blinded scorers, and average scores presented as average units. (G) Assessment of $\alpha$-SMA immunohistochemistry, red= $\alpha$-SMA, scale bar $=1 \mu \mathrm{m}$. (F) Quantification of $\alpha$-SMA immunoreactivity. Bronchioles of similar size ( $n=3 /$ mouse) were analyzed by two independent blinded scorers, and average scores presented as average units. Data represent 8 mice/group/time point. ${ }^{*} p<0.05$ (ANOVA) compared to the PBS group at the same time point. ${ }^{\dagger} \mathrm{p}<0.05$ (ANOVA, Kruskal Wallis) compared to WT mice exposed to HDM.

HDM-induced activation of the classical and alternative NF-KB pathways in human nasal and bronchial epithelial cells. Since we demonstrated increases in nuclear RelB in the bronchial epithelium in response to HDM administration, we sought to determine whether the alternative NF-KB pathway could be activated directly by HDM in human epithelial cells. Results in Fig. 6A demonstrate increased levels of NIK and p52 in response to HDM in Primary Human Nasal Epithelial Cells (PHNEC) obtained from two independent donors, and similar increases were observed (Fig. 6B) in human bronchial epithelial cells (HBEC), albeit less robust, demonstrating activation of the alternative NF-KB pathway. In addition, phosphorylation of RelA at serine 536, a separate parameter of NF-KB activation, was also increased in response to HDM (Fig. $6 \mathrm{~A}+\mathrm{B}$ ), demonstrating that in addition to the known ability of HDM to activate the classical NF-KB pathway, it also induces activation of the alternative NF-KB pathway in lung epithelial cells.
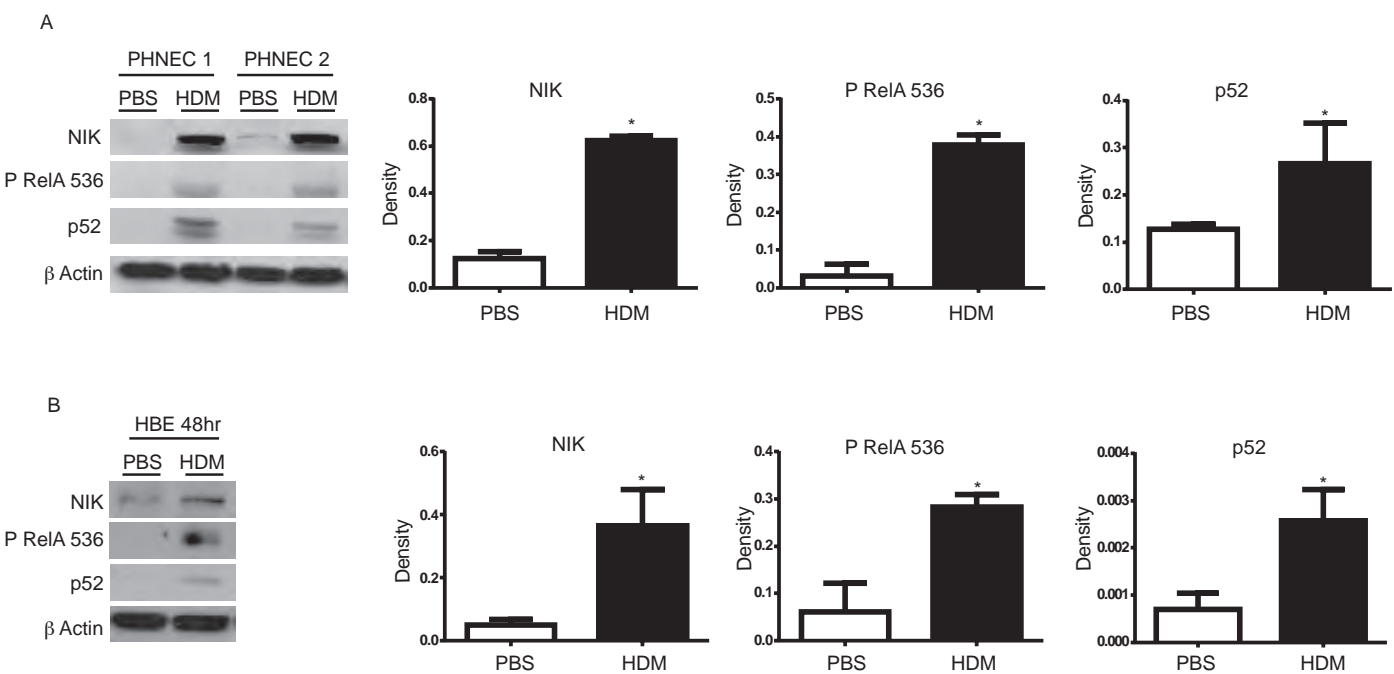
Figure 6. Assessment of activation of classical and alternative NF-KB pathways in human lung epithelial cells exposed to HDM. (A) Primary human nasal epithelial (PHNE) cells or (B) Human bronchial epithelial (HBE) cells were exposed to PBS or $25 \mu \mathrm{g}$ HDM once a day for consecutive days and harvested thereafter at either $72 \mathrm{~h}$ (PHNE) or $48 \mathrm{~h}$ (HBE). Cells were lysed for evaluation of NIK, p52, phospho RelA 536, by Western blot analyses. $\beta$-Actin (loading control). Right panels: Densitometric evaluation of Western blots shown in (A) PHNE ( $n=3$ patients) or (B) $\mathrm{HBE}(\mathrm{n}=3$ experimental repeats). Results are expressed as arbitrary density and were normalized to corresponding $\beta$-Actin bands.

\section{DISCUSSION}

NF-KB is a regulator of inflammation and immunity, and its role in the pathogenesis in asthma has been suggested based upon evidence of its activation in the bronchiolar epithelium from asthmatics [19] and from studies in mouse models of allergic airways disease [18]. Notably, studies previously performed in our laboratory have revealed a crucial role for lung epithelial NF-KB in the Alum/Ova model of allergic airways disease [9]. Moreover, transgenic expression of constitutively active IKK $\beta$ in lung epithelial cells was sufficient to cause neutrophilic inflammation and airways hyperresponsiveness, and enhanced sensitization to an inhaled antigen $[17,20]$. Despite these prior observations, the more generalized importance of epithelial NF-KB in allergic airways disease following sensitization/exposure to a relevant allergen via the airways has yet to be determined. Results presented herein describe a critical role for non-ciliated airway epithelial NF-KB in promoting inflammation, AHR, and fibrotic remodeling following extended challenges of HDM, while mucus metaplasia differences did not appear affected.

Results of the present study somewhat contrast our previous work using the Alum/Ova model of intraperitoneal sensitization followed by challenges of aerosolized Ova. Notably, CC10-IKB $\alpha_{S R}$ mice subjected to the Alum/Ova protocol were strongly protected against the development of eosinophilic inflammation and mucus metaplasia, but were not protected against Ova-induced AHR [9]. The reasons for these discrepant findings remain unclear. It is likely that the route of sensitization of an allergen dictates the nature of the subsequent immunological and pathophysiological response. In support of the latter is a previous report demonstrating that the IP sensitization regimen with Alum/Ova triggers eosinophilic, Th2-driven inflammation [21]. In contrast, sensitization with Ova via the airways, along lipopolysaccharide as the adjuvant, led to neutrophilic-dependent AHR and Th17-associated inflammation [22].

In the present study we demonstrated that $\mathrm{CC} 10-\mathrm{IKB}_{\mathrm{SR}}$ mice did not display increases in airway neutrophils following 15 challenges with HDM compared to PBS controls, while neutrophils were robustly increased in BAL of WT mice. In contrast, HDM-induced eosinophilia was only partially 
attenuated in $\mathrm{CC} 10-\mathrm{IKB} \alpha_{S R}$ mice, while BAL lymphocytes were not significantly affected. IL-5, a potent eosinophil chemoattractant in mice and humans, was not detectable at any experimental time points (data not shown); however, we did demonstrate a strong decrease in KC (human IL-8) in CC10-IKB $\alpha_{S R}$ mice in comparison to WT mice exposed to HDM. These findings demonstrate that following inhibition of epithelial NF-KB in the setting of HDM-induced disease, the inflammatory process is not uniformly inhibited, but preferentially affects neutrophils. This potential differential effect of NF-KB inhibition on the inflammatory process could explain the impact of airway remodeling and AHR. Consistent with the continued presence of eosinophils, HDM-specific IgG1 and IgE, and IL-13 in CC10-IKB $\alpha_{S R}$ mice exposed to HDM, mucus metaplasia and Muc5AC expression remained elevated in these animals. Previous studies demonstrated that IL-13 can activate epithelial cells to produce Muc5ac in an NF-KB independent mechanism [23]. In light of those observations, the lack of an impact of CC10-I-KBaSR mice in HDMinduced mucus metaplasia is therefore, perhaps, not surprising.

The role of neutrophils in the pathogenesis of asthma remains unclear. HDM exposure in mice has been associated with mixed neutrophilic/eosinophilic, Th2/Th17-linked inflammation, and production of IL-13 and IL-17A [24]. In this study, the preferential diminution of neutrophils following inhibition of epithelial NF-KB was associated with normalization of tissue damping and elastance parameters towards values observed in controls, while in contrast, Newtonian resistance, reflective of the central airways, was unaffected. The disparate effects of the $\mathrm{CC} 10-\mathrm{IKB}_{\mathrm{SR}}$ transgene on tissue damping and elastance, as compared to Newtonian resistance, are puzzling. Previous studies have suggested that inflammation contributed to enhanced leakage of fibrin to the airway surface, leading to decreased stability of surfactant proteins and increased surface tension, and facilitated contractility of smooth muscle. These mechanisms have been linked to increases in the closure of distal airways [2527]. It is plausible that decreases in inflammation, along with decreased $\alpha$-smooth muscle actin in bronchioles, account for decreases in tissue damping and elastance, which were observed in CC10$\mathrm{I} B \mathrm{~B} \alpha_{\mathrm{SR}}$ transgenic mice. This putative explanation would need to be addressed with additional studies. Our data also demonstrate that KC, a potent neutrophil chemokine, was not increased in CC10-IKB $\alpha_{S R}$ HDM-exposed mice in comparison to PBS controls, indicating a potential mechanism whereby neutrophils are decreased in $\mathrm{CC} 10-\mathrm{IKB}_{\mathrm{SR}}$ mice. All together, these findings are suggestive of a role for neutrophils in promoting increases in peri-bronchiolar collagen deposition and associated changes in tissue damping and elastance. Alternatively, it is also plausible that other mediators control neutrophil trafficking to the airways, promote peri-bronchiolar remodeling, and changes in AHR. In this regard, IL$17 \mathrm{~A}$, which we also demonstrate to be decreased in the tissue of $\mathrm{CC} 10-\mathrm{I} K B \alpha_{\mathrm{SR}}$ mice, has been shown to 
stimulate production of chemokines important in neutrophil recruitment in a KC-dependent manner [28] and has been implicated in the pathogenesis of pulmonary fibrosis [29], yet its functional contribution here remains to be determined. Additionally, eosinophils expressing TGF- $\beta 1$ have been shown to be important in allergen-induced peribronchial fibrosis [30], and hence it is possible that TGF$\beta 1$ also plays a role in peri-bronchiolar fibrosis downstream of activation of NF-kB. Additional studies will be required to formally address these putative scenarios.

In addition to demonstrating a role for epithelial NF-KB in promoting inflammation, AHR, and fibrosis following 15 challenges of HDM, we also established a contributing role for epithelial NF-KB in regulating pro-inflammatory gene expression following a single exposure of HDM. Numerous proinflammatory mediators have been implicated in the development of allergic airways disease [3], and our studies demonstrate a putative role for epithelial NF-KB in regulating expression of several of these molecules in response to HDM. GM-CSF and CCL20 have been shown to be important in the recruitment/activation of dendritic cells, which are required for $T$ cell activation and recognition of antigens. Notably, exposure of human asthmatic bronchial epithelial cells to the HDM component, Derp1, was shown to be important in dendritic cell recruitment in a CCL20 dependent manner [31]. Additionally, IL-33 has been shown to be important in the activation of a variety of cell types crucial to the development of asthma, such as T helper type 2 cells, eosinophils, dendritic cells, and mast cells [32]. As previously mentioned, the activation and infiltration of neutrophils is emerging as a potential phenotype in severe, steroid resistant asthma. Cytokines KC and MIP-2 have both been shown to be important in the recruitment of neutrophils and production of HDM-specific IgE [33]. Altogether, our data indicate a likely role for epithelial NF-KB in the recruitment/activation of several cell types important for the development of HDM-induced asthma. This suggests a mechanism whereby epithelial NF-KB activation is the crucial step between contact with an allergen and downstream manifestations of asthma.

In addition to the role of NF-KB in airway epithelium demonstrated herein, it is plausible that activation NF-KB in other cell types contributes to the pathophysiology of allergic airways disease. Notably our findings demonstrate increased immunofluorence of nuclear RelA and RelB in parenchymal regions following exposure to HDM (Fig. 3). Unraveling the cell types in which NF-KB is activated and their functional contribution to allergic airways disease would require additional cell specific labeling and targeting strategies, which were beyond the scope of the present study. For example, neutrophil elastase-induced secretion of Transforming Growth Factor $\beta-1$ from smooth muscle cells was shown to be dependent on NF-KB activation [34], and the smooth muscle contractile force in response to IL-17A 
was dependent on NF-KB activation [24], suggesting a putative role of NF-KB activation in smooth muscle cells in airways hyperresponsiveness and remodeling. Secretion of eotaxin, a potent eosinophil activating factor, by fibroblasts was also shown to be dependent on NF-kB activation [35]. One notable finding of the present study is that HDM activates both the classical and alternative NF-KB pathway within the bronchiolar epithelium, evidenced by the increases in nuclear presence of both RelA as well as RelB in mice exposed to HDM. Similar to observations herein, we recently demonstrated increases in nuclear RelA and RelB in the parenchymal regions following administration of lipopolysaccharide, a component of HDM [7], suggesting that TLR4 activation by HDM may be contributing to the observed increases in RelA and RelB observed. Increases in nuclear RelA and RelB within the epithelium occurred rapidly and were sustained at least for $24 \mathrm{~h}$ after 3 challenges. However, $72 \mathrm{~h}$ post 5, 10 or 15 challenges, there was no clear evidence of increased RelA or RelB in the bronchiolar epithelium, possibly due to the timing of tissue analysis post the last administration of HDM, which was $72 \mathrm{~h}$, a time when NF-KB activation may have resolved. However, we did observe sustained increases in nuclear RelB in lung tissue homogenates $72 \mathrm{hr}$ post 15 challenges with HDM in WT mice, suggestive of NF-KB activation in other cell types. Increases in nuclear RelB were attenuated in $\mathrm{CC} 10-\mathrm{I} \mathrm{KB} \mathrm{S}_{\mathrm{SR}}$ mice, suggesting a putative role for RelB in the orchestration of HDM-mediated AHR and fibrotic remodeling. In addition to the demonstration that RelB was increased in the bronchiolar epithelium following HDM exposure, we also demonstrated that HDM directly activated both NF-KB pathways in both human bronchial and nasal epithelial cells. Although extensive studies have been conducted to unravel the molecular regulation and pathophysiological relevance of the classical NF-KB pathway, far less information is available for the alternative pathway. The latter pathway was originally thought to play a role in adaptive immune responses, development of lymphocytes and lymphoid organs. However, emerging studies have pointed to a coordinate function of both classical and alternative NF-KB in the orchestration of pro-inflammatory responses. For example, exposure of lung epithelial cells with Tumor Necrosis Factor- $\alpha$, Polyinosinic acid, LPS, IL-17A, lipoteichoic acid, and CD40L, agonists that signal through distinct families of receptors, led to a coordinate activation of classical and alternative NF-KB pathways and subsequent proinflammatory responses [7]. In contrast, adenovirus-mediated delivery of RelB afforded protection against cigarette smoke-induced neutrophilic inflammation [36], suggesting potentially complex roles of the alternative NF-KB pathway in the regulation of pro-inflammatory and immune responses. The classical NF-KB pathway previously has been shown to be important in HDM-mediated pro-inflammatory responses in human bronchial epithelial cells in vitro [12], and Derp1, a component of HDM, was shown to be important in the activation of NF-KB in human asthmatic bronchial epithelial cells [37]. Patients 
with allergic asthma demonstrate increased classical NF-KB activation in nasal epithelial cells in response to HDM in comparison to healthy controls [38]. Intriguingly, the HDM component, $\beta$-glucan, was shown to be crucial for activation of allergic rhinitis in nasal epithelial cells via TLR2, in contrast to another HDM component, LPS, which is important in promoting allergic airways disease via activation of TLR4 in bronchial epithelial cells [39]. Despite these previous studies and data presented herein, additional studies are needed to better understand the timing and locale of activation of classical and alternative NF-KB pathways, the components of HDM that are required to activate either pathway, as well as the relative contributions of the classical and alternative NF-KB pathways in eliciting HDM- triggered allergic airways disease.

In summary, we demonstrate in the present study the importance of NF-KB activation within the bronchiolar epithelium in HDM-induced inflammation, AHR and fibrotic airway remodeling. We also demonstrate that both classical and alternative NF-KB pathways are activated by HDM. Data presented herein showed that in the setting of HDM-induced allergic airway disease, inhibition of epithelial NF-KB plays a more prominent role in attenuating neutrophilia, AHR, and remodeling, in comparison to eosinophilia, IgE, and mucus metaplasia. Therefore, it is plausible that therapeutic approaches which target NF-KB via interference with degradation of IKB $\alpha$ may have a stronger impact on asthmatic patients with predominant neutrophila in contrast to patients with predominant eosinophilia. Corticosteroids, the most common therapy for asthma, inhibit NF-KB, but have many off target effects. Current therapies that are being developed for asthma are aimed at inhibiting the pro-inflammatory effects of NF-KB signalling in the lung [40], and are focused on inhibition of IKK $\beta$, the dominant kinase in the classical NF-KB pathway [40]. The small molecule IKK $\beta$ inhibitor, IMD-0354, attenuated HDMinduced eosinophilia, globlet cell hyperplasia, subepithelial fibrosis, smooth muscle cell hypertrophy, and lung resistance using an intraperitoneal senstization model [41]. The disparate findings of the latter study with the present findings may relate to the different sensitization route, mechanism of inhibition of NF-kB, and the cell types wherein NF-kB inhibition occurred. Furthermore, intravenous administration of RelA antisense oligonucleotides prior to ovalbumin challenge resulted in dampened responses of inflammation, $\mathrm{AHR}$, and $\mathrm{TH} 2$ responses in mice [42]. Based upon these collective findings, therapeutics designed to inhibit both facets of the NF-kB pathway may hold larger therapeutic potential for allergic asthma and other allergic diseases of the lung. 


\section{REFERENCES}

1. Hayden, M.S. and S. Ghosh, Signaling to NF-kappaB. Genes Dev, 2004. 18(18): p. 2195-224.

2. Scheidereit, C., IkappaB kinase complexes: gateways to NF-kappaB activation and transcription. Oncogene, 2006. 25(51): p. 6685-705.

3. Swamy, M., et al., Epithelial decision makers: in search of the 'epimmunome'. Nat Immunol, 2010. 11(8): p. 656-65.

4. Senftleben, U., et al., Activation by IKKalpha of a second, evolutionary conserved, NF-kappa B signaling pathway. Science, 2001. 293(5534): p. 1495-9.

5. Oeckinghaus, A., M.S. Hayden, and S. Ghosh, Crosstalk in NF-kappaB signaling pathways. Nat Immunol, 2011. 12(8): p. 695-708.

6. Xiao, G., et al., Alternative pathways of NF-kappaB activation: a double-edged sword in health and disease. Cytokine Growth Factor Rev, 2006. 17(4): p. 281-93.

7. Tully, J.E., et al., Cooperation between classical and alternative NF-kappaB pathways regulates proinflammatory responses in epithelial cells. Am J Respir Cell Mol Biol, 2012. 47(4): p. 497-508.

8. Poynter, M.E., C.G. Irvin, and Y.M. Janssen-Heininger, A prominent role for airway epithelial NFkappa B activation in lipopolysaccharide-induced airway inflammation. J Immunol, 2003. 170(12): p. 6257-65.

9. Poynter, M.E., et al., NF-kappa B activation in airways modulates allergic inflammation but not hyperresponsiveness. J Immunol, 2004. 173(11): p. 7003-9.

10. Broide, D.H., et al., Allergen-induced peribronchial fibrosis and mucus production mediated by IkappaB kinase beta-dependent genes in airway epithelium. Proc Natl Acad Sci U S A, 2005. 102(49): p. 17723-8.

11. Gregory, L.G. and C.M. Lloyd, Orchestrating house dust mite-associated allergy in the lung. Trends Immunol, 2011. 32(9): p. 402-11.

12. Osterlund, C., et al., The non-proteolytic house dust mite allergen Der $p 2$ induce NF-kappaB and MAPK dependent activation of bronchial epithelial cells. Clin Exp Allergy, 2009. 39(8): p. 1199208.

13. Olson, N., et al., Nitric oxide and airway epithelial barrier function: regulation of tight junction proteins and epithelial permeability. Arch Biochem Biophys, 2009. 484(2): p. 205-13.

14. Wu, R., Y.H. Zhao, and M.M. Chang, Growth and differentiation of conducting airway epithelial cells in culture. Eur Respir J, 1997. 10(10): p. 2398-403.

15. Jaspers, I., et al., Diesel exhaust enhances influenza virus infections in respiratory epithelial cells. Toxicol Sci, 2005. 85(2): p. 990-1002.

16. Riesenfeld, E., et al., The Temporal Evolution of Airways Hyperresponsiveness and Inflammation. J Allergy Ther, 2012. 1(5): p. 1-7.

17. Pantano, C., et al., Nuclear factor-kappaB activation in airway epithelium induces inflammation and hyperresponsiveness. Am J Respir Crit Care Med, 2008. 177(9): p. 959-69.

18. Poynter, M.E., C.G. Irvin, and Y.M. Janssen-Heininger, Rapid activation of nuclear factor-kappaB in airway epithelium in a murine model of allergic airway inflammation. Am J Pathol, 2002.

160(4): p. 1325-34.

19. Zhao, S., et al., Activation of NF-kappa B in bronchial epithelial cells from children with asthma. Chin Med J (Engl), 2001. 114(9): p. 909-11.

20. Ather, J.L., et al., Airway epithelial NF-kappaB activation promotes allergic sensitization to an innocuous inhaled antigen. Am J Respir Cell Mol Biol, 2011. 44(5): p. 631-8.

21. Kung, T.T., et al., Characterization of a murine model of allergic pulmonary inflammation. Int Arch Allergy Immunol, 1994. 105(1): p. 83-90. 
22. Wilson, R.H., et al., Allergic sensitization through the airway primes Th17-dependent neutrophilia and airway hyperresponsiveness. Am J Respir Crit Care Med, 2009. 180(8): p. 720-30.

23. Whittaker, L., et al., Interleukin-13 mediates a fundamental pathway for airway epithelial mucus induced by CD4 T cells and interleukin-9. Am J Respir Cell Mol Biol, 2002. 27(5): p. 593-602.

24. Kudo, M., et al., IL-17A produced by alphabeta $T$ cells drives airway hyper-responsiveness in mice and enhances mouse and human airway smooth muscle contraction. Nat Med, 2012. 18(4): p. 547-54.

25. Hohlfeld, J.M., The role of surfactant in asthma. Respir Res, 2002. 3: p. 4.

26. Wagers, S.S., et al., Extravascular fibrin, plasminogen activator, plasminogen activator inhibitors, and airway hyperresponsiveness. J Clin Invest, 2004. 114(1): p. 104-11.

27. Yager, D., R.D. Kamm, and J.M. Drazen, Airway wall liquid. Sources and role as an amplifier of bronchoconstriction. Chest, 1995. 107(3 Suppl): p. 105S-110S.

28. Laan, M., et al., Neutrophil recruitment by human IL-17 via C-X-C chemokine release in the airways. J Immunol, 1999. 162(4): p. 2347-52.

29. Hasan, S.A., et al., Role of IL-17A and neutrophils in fibrosis in experimental hypersensitivity pneumonitis. J Allergy Clin Immunol, 2013.

30. Cho, J.Y., et al., Inhibition of airway remodeling in IL-5-deficient mice. J Clin Invest, 2004. 113(4): p. 551-60.

31. Pichavant, M., et al., Asthmatic bronchial epithelium activated by the proteolytic allergen Der $p 1$ increases selective dendritic cell recruitment. J Allergy Clin Immunol, 2005. 115(4): p. 771-8.

32. Borish, L. and J.W. Steinke, Interleukin-33 in asthma: how big of a role does it play? Curr Allergy Asthma Rep, 2011. 11(1): p. 7-11.

33. McKinley, L., et al., CXC chemokines modulate IgE secretion and pulmonary inflammation in a model of allergic asthma. Cytokine, 2005. 32(3-4): p. 178-85.

34. Lee, K.Y., et al., Neutrophil-derived elastase induces TGF-beta1 secretion in human airway smooth muscle via NF-kappaB pathway. Am J Respir Cell Mol Biol, 2006. 35(4): p. 407-14.

35. Rokudai, A., et al., Differential regulation of eotaxin-1/CCL11 and eotaxin-3/CCL26 production by the TNF-alpha and IL-4 stimulated human lung fibroblast. Biol Pharm Bull, 2006. 29(6): p. 11029.

36. McMillan, D.H., et al., Lung-targeted overexpression of the NF-kappaB member RelB inhibits cigarette smoke-induced inflammation. Am J Pathol, 2011. 179(1): p. 125-33.

37. Stacey, M.A., et al., The allergen Der 1 induces NF-kappaB activation through interference with IkappaB alpha function in asthmatic bronchial epithelial cells. Biochem Biophys Res Commun, 1997. 236(2): p. 522-6.

38. Vroling, A.B., et al., Primary nasal epithelium exposed to house dust mite extract shows activated expression in allergic individuals. Am J Respir Cell Mol Biol, 2008. 38(3): p. 293-9.

39. Ryu, J.H., et al., Distinct TLR-mediated pathways regulate house dust mite-induced allergic disease in the upper and lower airways. J Allergy Clin Immunol, 2013. 131(2): p. 549-61.

40. Edwards, M.R., et al., Targeting the NF-kappaB pathway in asthma and chronic obstructive pulmonary disease. Pharmacol Ther, 2009. 121(1): p. 1-13.

41. Ogawa, H., et al., IkappaB kinase beta inhibitor IMD-0354 suppresses airway remodelling in a Dermatophagoides pteronyssinus-sensitized mouse model of chronic asthma. Clin Exp Allergy, 2011. 41(1): p. 104-15.

42. Choi, I.W., et al., Administration of antisense phosphorothioate oligonucleotide to the p65 subunit of NF-kappaB inhibits established asthmatic reaction in mice. Int Immunopharmacol, 2004. 4(14): p. 1817-28. 


\section{CHAPTER 4}

Genetic ablation of glutaredoxin- 1 causes enhanced resolution of enhanced airways hyperresponsiveness and mucus metaplasia in mice with allergic airways disease

Sidra M. Hoffman, Jane E. Tully, Karolyn G. Lahue, Vikas Anathy, James D. Nolin, Amy S. Guala, Jos L. van der Velden, Yi Shi Ho, Minara Aliyeva, Nirav Daphtary, Lennart K. Lundblad, Charles G. Irvin, and Yvonne M.W. Janssen-Heininger

American Journal of Physiology Lung Cell Molecular Physiology 2012; 303. L528-38 


\section{ABTRACT}

Protein-S-glutathionylation (PSSG) is an oxidative modification of reactive cysteines that has emerged as an important player in patho-physiological processes. Under physiological conditions, the thiol transferase, glutaredoxin-1 (Glrx1) catalyses de-glutathionylation. Although we previously demonstrated that GIrx1 expression is increased in mice with allergic inflammation, the impact of Glrx1/PSSG in the development of allergic airways disease remains unknown. In the present study we examined the impact of genetic ablation of Glrx1 in the pathogenesis of allergic inflammation and airway hyperresponsiveness (AHR) in mice. GIrx $1^{\%}$ or WT mice were subjected to the antigen, ovalbumin (OVA) and parameters of allergic airways disease were evaluated $48 \mathrm{hr}$ after 3 challenges, and $48 \mathrm{hr}$ or 7 days after 6 challenges with aerosolized antigen. Although no clear increases in PSSG were observed in WT mice in response to OVA, marked increases were detected in lung tissue of mice lacking G/rx1 $48 \mathrm{hr}$ following 6 antigen challenges. Inflammation and expression of pro-inflammatory mediators were decreased in G/rx $1^{\%}$ mice, dependent on the time of analysis. WT and Glrx $1^{-}$mice demonstrated comparable increases in AHR $48 \mathrm{hr}$ after 3 or 6 challenges with OVA. However, 7 days post cessation of 6 challenges, parameters of AHR in GIrx $1^{\%}$ mice were resolved to control levels, accompanied by marked decreases in mucus metaplasia and expression of Muc5AC and GOB5. These results demonstrate that the GIrx1/S-glutathionylation redox status in mice is a critical regulator of AHR, suggesting that avenues to increase $S$-glutathionylation of specific target proteins may be beneficial to attenuate AHR.

\section{Keywords}

Asthma, lung inflammation, protein S-glutathionylation 


\section{INTRODUCTION}

Chronic inflammatory disorders of the lung, including asthma, are accompanied by changes in the oxidative environment [1]. For instance, oxidative inactivation of the antioxidant enzymes copper zinc containing superoxide dismutase and manganese superoxide dismutase, have been demonstrated in patients with asthma $[2,3]$, and changes in the redox status of the tri-peptide antioxidant, glutathione (GSH) have also been reported in patients with asthma compared with healthy controls $[4,5]$. The exact mechanisms whereby oxidants contribute to disease pathogenesis remain unresolved. GSH, which is highly abundant and acts as an antioxidant and reducing agent in cells in lung lining fluid, plays a major role in maintenance of overall redox homeostasis. Consequently, agents that cause oxidative stress are known to decrease the ratio of reduced GSH to oxidized glutathione (GSSG, glutathione disulfide) [6]. In addition to changes in reduced to oxidized glutathione ratio, under conditions of oxidative stress, glutathione can become conjugated to reactive cysteines in proteins, a post-translational modification known as protein S-glutathionylation (PSSG, also known as S-glutathiolation, or protein mixed disulfides). PSSG is a redox-based post-translational modification of proteins that changes structure and function of proteins in a reversible and tightly regulated manner [7, 8]. Mammalian glutaredoxin (GIrx) enzymes are members of the thioredoxin family of thiol transferases that, under physiological conditions, specifically catalyze de-glutationylation reactions, leading to the restoration of the reduced sulfhydryl group of protein cysteines [9].

The functional significance of altered regulation of protein function through PSSG in allergic airways disease remains unknown. We recently demonstrated increases in Glrx1 expression and increases in Glrx activity in lung tissue from mice with antigen-induced allergic inflammation. Increases in Glrx1 immunoreactivity occurred predominantly in the bronchiolar epithelium of mice with allergic airways inflammation [10]. Furthermore, in mice lacking the glutaredoxin-1 gene (G/rx $\left.1^{\%}\right)$, lipopolysaccharide (LPS)-induced inflammation and macrophage activation were attenuated, in association with enhanced S-glutathionylation [11]. Primary lung epithelial cells derived from Glrx $1^{-1}$ mice also showed markedly attenuated production of inflammatory mediators in response to LPS compared with epithelial cells isolated from wild-type (WT) mice [12]. The goal of the present study was to determine the impact of Glrx1 ablation on the development of allergic airways disease, using the well characterized model of ovalbumin-induced $\mathrm{T}_{\mathrm{H}} 2$ inflammation and airway hyperresponsiveness (AHR). Our results demonstrate that $\mathrm{GIr \times 1} \mathbf{1}^{\%}$ mice showed enhanced resolution of AHR and mucus metaplasia, in association with increases in PSSG, suggesting a role for PSSG in ameliorating AHR. 


\section{MATERIALS AND METHODS}

Glrx $1^{\%}$ mice were backcrossed for more than 10 generations into a BALB/c background [13] (The Jackson Laboratories, Bar Harbor, ME) and were housed in the University of Vermont Animal Facility. For all experiments, eight- to twelve-week-old $\mathrm{Glr} \mathrm{1}^{\%}$ and littermate WT BALB/c control mice were used, as approved by the Institutional Animal Care and Use Committee.

Ovalbumin model of allergic airway disease. WT BALB/c and Glrx $1^{\%}$ mice were administered $20 \mu \mathrm{g}$ of the antigen, OVA (Grade V, Sigma Aldrich, St. Louis, MO) with aluminum hydroxide (alum) (Pierce, Rockford, IL) via an intraperitoneal injection on days 0 and 14 as previously described $[14,15]$. The control group was mock sensitized, receiving intraperitoneal phosphate-buffered saline (PBS) and alum. All groups of mice were challenged using 3 or 6 doses of aerosolized 1\% OVA in sterile PBS for 30 min on days 21, 22, 23 [16], or on days 21-26. The animals were harvested $48 \mathrm{hr}$ or 7 days post challenge.

Assessment of airway hyperresponsiveness. Mice were anesthetized with i.p. sodium pentobarbital $(90 \mathrm{mg} / \mathrm{kg})$, tracheotomized, and mechanically ventilated at 200 breaths/min using a flexiVent $^{\mathrm{TM}}$ computer controlled small animal ventilator (SCIREQ, QC, Canada), as previously described [14, 17]. Airways responsiveness was quantified by averaging the three highest measurements obtained at each incremental methacholine dose.

Bronchoalveolar lavage (BAL) and lung processing. BAL (800-1000 $\mu \mathrm{l})$ was collected from mice immediately after the completion of measurements of respiratory mechanics. Total and differential cell counts were performed as previously described [14]. Briefly, cells were isolated by centrifugation and total cell counts were performed using the Advia 120 automated hematology analyzer system. Cytospins were performed and stained using the Hema3 kit (Fisher Scientific, Kalamazoo, MI). Differential cell counts were performed on a minimum of 300 cells. Right lung lobes were flash frozen for RNA and protein isolation. Left lung lobes were fixed with $4 \%$ paraformaldehyde, mounted in paraffin, and $5-\mu \mathrm{m}$ sections were prepared for histopathology as previously described [16].

Cytokineprofiling. BAL samples were thawed and assayed for OVA-specific immunoglobulins and inflammatory cytokine levels as previously described [11]. Enzyme-Linked Immunosorbent assays (ELISA) were utilized to assess cytokine content in undiluted BAL according to manufacturer's instructions (R \& 
D Systems, Minneapolis, MN). For simultaneous quantitation of multiple analytes, undiluted BAL fluid or serum was analyzed using a mouse cytokine 23-plex kit on the Bio-Plex suspension array system (BioRad, Hercules, CA) [18].

mRNA analysis. Total RNA was isolated from pulverized lung tissue using the RNeasy kit (Qiagen, Valencia, CA). $1 \mu \mathrm{g}$ of RNA was used for cDNA synthesis and reverse transcribed for Taqman gene analysis using SYBR green (Bio-Rad, Hercules, CA). Primers for quantitative reverse transcriptase polymerase chain reaction include: KC (Fwd:5'-GCTGGATTCACCTCAAGAA-3', Rv:5'TGGGGACACCTTTTAGCATC-3'), RANTES (Fwd:5'-ATATGGCTCGGACACCACTC-3', Rv:5'tCCTTCGAGTGACAAACACG-3'), IL-6 (Fwd:5'-CTGATGCTGGTGACAACCAC-3', Rv:5'CAGAATtGCCATTGCACAAC-3'), CCL20 (Fwd:5'-AAGACAGATGGCCGATGAAG-3', Rv:5'-

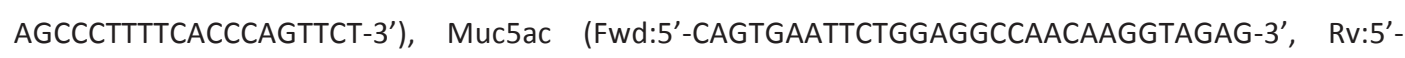

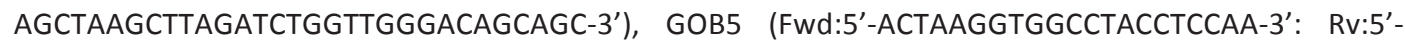
GGAGGtgacAGtCAAGgtgagA-3'), and IL-5 (Fwd: 5'-ATGGAGATtCCCATGAGCAC-3', Rv:5'CCCACGGACAGTTTGATTCT-3') The fold induction was calculated using the housekeeping gene, cyclophilin.

Histopathology. Periodic acid Schiff staining was performed, and mucus metaplasia was evaluated by obtaining three images of small bronchioles (20X objective) of similar dimensions from each mouse. Images were blinded and ranked by two independent investigators, at a scale from 0 to $3 ; 0$, being no reactivity; 1 , minimal staining; 2 , moderate staining; 3 , prominent staining. The cumulative score from each mouse was then averaged according to treatment group.

Biochemical analysis of protein S-glutathionylation in lung tissue. Protein S-glutathionylation in lung tissue was determined using the glutathione/glutathione reductase/NADPH/ 5,5'-dithiobis (2nitrobenzoic acid) (DTNB) recycling assay, according to procedures described previously [19]. In brief, pulverized lung tissue stored at $-80^{\circ} \mathrm{C}$ was homogenized in buffer containing $137 \mathrm{mM}$ Tris- $\mathrm{HCl}, \mathrm{pH} 8.0$, $130 \mathrm{mM} \mathrm{NaCl}$, and 1\% NP-40. Protein content was determined, and samples were equalized for protein content. $200 \mu \mathrm{g}$ of protein was precipitated with acetone. The pellet was resuspended in $0.1 \%$ TritonX100, 0.6\% sulfasalicylic acid containing buffer, and freeze thawed twice. Protein-associated glutathione was released with sodium borohydride, and GSH determined. Samples that were not treated with 
sodium borohydride were used as a control. The sodium borohydride sensitive fraction of GSH was calculated, and expressed as nmol GSH/mg of protein.

Statistical analyses. Analyses of all data were performed using the Graph Pad Prism software (Graphpad Inc.) by ANOVA, or Student's $t$ test where appropriate. All experiments were repeated twice, and data from combined experiments are presented plus/minus the standard error of the mean (SEM). Histological scoring was analyzed using the Kruskal-Wallis test, and Dunn's multiple comparison posthoc tests. Analyses with resultant $p$ values of $<0.05$ were accepted as significant.

\section{RESULTS}

Variable impact of Glrx1 ablation on OVAlbumin-induced airways inflammation. In order to model allergic airways disease, we utilized the well described model of OVA-induced pulmonary inflammation to produce $\mathrm{T}_{\mathrm{H}} 2$-dominated allergic airways disease. WT and $\mathrm{G} / r \times 1^{-}$mice were immunized with OVA plus alum on days 0 and 14, and challenged with aerosolized OVA on days 21, 22, 23 and harvested $48 \mathrm{hr}$ later ( 3 Chall.+ 48hr). In addition, separate groups of mice were immunized, and then subjected to 6 consecutive OVA challenges and harvested $48 \mathrm{hr}(6$ Chall. $+48 \mathrm{hr}$ ) and 7 days (6 Chall.+ $7 \mathrm{~d}$ ) post final challenge. We first assessed OVA-specific immunoglobulins in serum from WT and Glrx $1^{-/}$mice following sensitization and challenge. Results in Fig. 1 demonstrate comparable increases in levels of IgE (Fig. 1A) and IgG1 (Fig. 1B) in WT and GIrx $1^{\%}$ mice subjected to sensitization and challenge with OVA, compared to alum/OVA controls, demonstrating that $G / r \times 1 \%$ mice mounted an equally robust immune response to OVA as WT mice. 

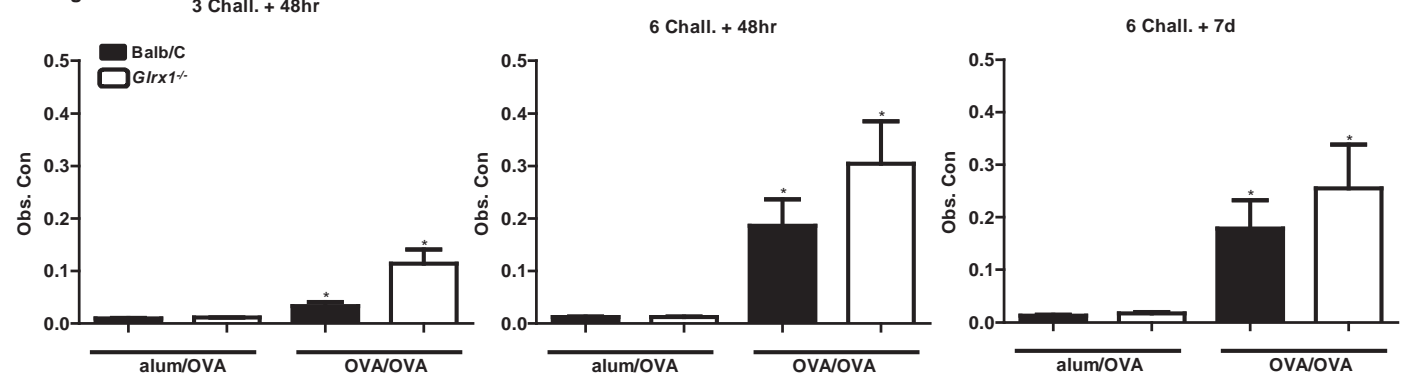

B. $\lg G 1$
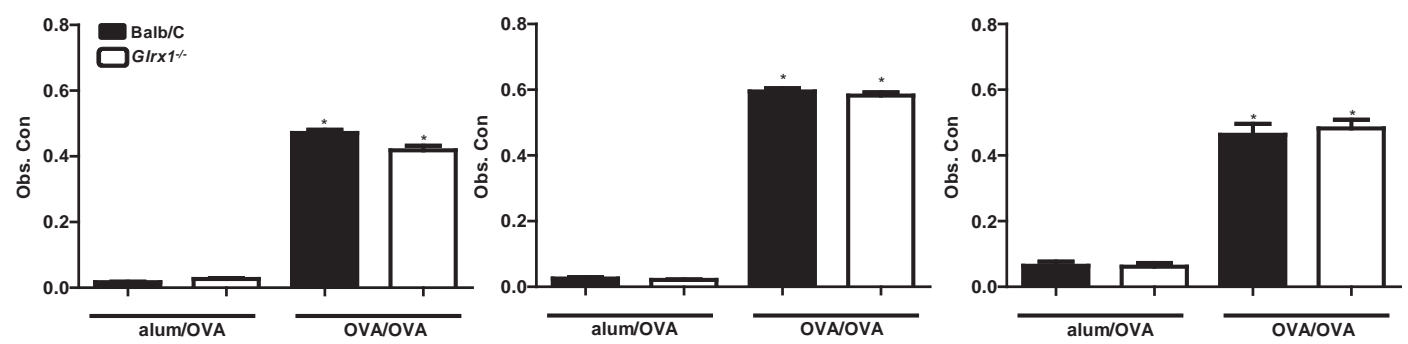

Figure 1. Assessment of OVA-specific Immunoglobulins in WT or Glrx $1^{-1}$ mice following sensitization and challenge with OVA. Serum immunoglobulin levels were measured by ELISA. Standard curves for (A) OVA-specific $\operatorname{lgE}$ and (B) IgG1 were generated from alum/OVA-sensitized BALB/c mouse serum. For comparisons, values for the most concentrated were set to 10,000 units $/ \mathrm{ml}$. Data are expressed as means \pm SEM from 8 to 10 mice per group. ${ }^{*} P<0.05$ (ANOVA) compared with respective alum/OVA control groups. Black bars: BALB/c mice, white bars: Glrx1 /- mice.

We next assessed the magnitude of airways inflammation in WT and GIrx $1^{-/}$mice in response to OVA by enumerating cells obtained via BAL. All mice immunized and challenged with OVA showed a marked influx of cells into the airways (Fig. 2), characterized by increases in eosinophils, and to lesser but significant extent, neutrophils and lymphocytes (Table 1). Inflammation was most robust $48 \mathrm{hr}$ after 6 challenges, and remained strongly elevated, despite 7 days of recovery post final challenge. Genetic ablation of $\mathrm{G} / \mathrm{r} \times 1^{-}$had a variable impact on OVA-induced inflammation. While inflammation was significantly decreased in the G/rx $1^{\text {\%- }}$ mice $48 \mathrm{hr}$ following 3 challenges with OVA, predominantly due to a lower influx of eosinophils, at the later time points, overall airway inflammation was comparable between WT and Glrx1\% mice (Fig. 2 and Table 1). 

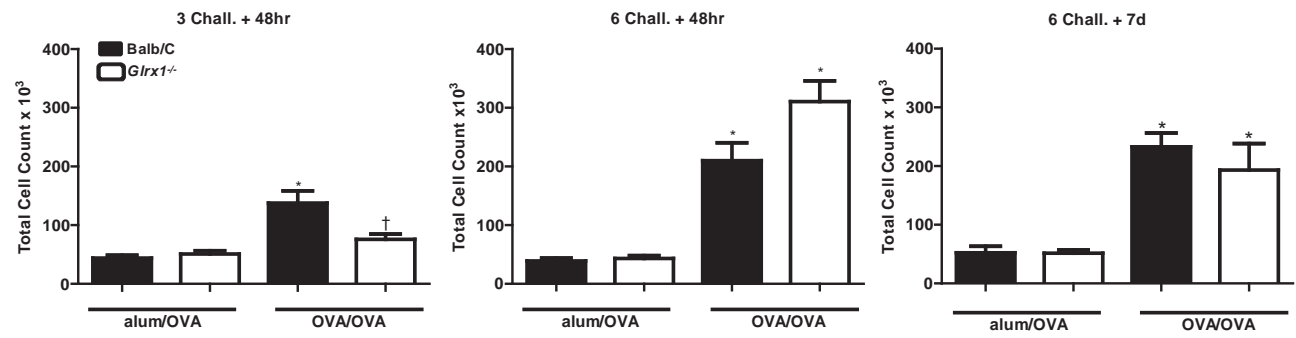

Figure 2. Total cell counts in BAL from WT or $\mathrm{G} / r \times 1^{\%}$ mice following sensitization and challenge with OVA. Control mice received an intraperitoneal injection of alum and PBS (alum/OVA) at days 0 and 14. Sensitized mice were administered an intraperitoneal injection of alum and OVA (OVA/OVA) at days 0 and 14. Both groups were nebulized with aerosolized OVA at days 21,22 , and 23, or days 21 through 26 . BAL was performed $48 \mathrm{hr}$ or 7 days after the last aerosolized OVA challenge. Total cell numbers were determined by Advia, and data are expressed as means \pm SEM from 8 to 10 mice per group. ${ }^{*} P<0.05$ (ANOVA) compared with respective alum/OVA control groups. Black bars: BALB/c mice, white bars: GIr $\times 1^{-1-}$ mice.

Table 1: BAL Cell Differentials

\begin{tabular}{|c|c|c|c|c|}
\hline 3 Chall. $+48 \mathrm{hr}$ & \multicolumn{2}{|c|}{ Balb/C } & \multicolumn{2}{|c|}{$\underline{G /} \times 1^{\prime}$} \\
\hline $10^{3}$ & alum/OVA & OVA/OVA & alum/OVA & OVA/OVA \\
\hline MACS & $42.2 \pm 5.4$ & $29.5 \pm 5.4$ & $52.4 \pm 5.4$ & $38.7 \pm 4.3$ \\
\hline EOS & $0.0 \pm 0.0$ & ${ }^{*} 93.2 \pm 14.8$ & $0.9 \pm 0.5$ & $*+27.7 \pm 5.4$ \\
\hline PMN & $0.8 \pm 0.3$ & $* 11.7 \pm 3.9$ & $1.2 \pm 0.3$ & $7.7 \pm 2.1$ \\
\hline LYMPH & $0.0 \pm 0.0$ & $* 3.1 \pm 0.5$ & $0.5 \pm 0.2$ & $1.7 \pm 0.4$ \\
\hline 6 Chall. $+48 \mathrm{hr}$ & \multicolumn{2}{|c|}{ Balb/C } & \multicolumn{2}{|c|}{$\underline{G / r \times 1^{1 /}}$} \\
\hline $10^{3}$ & alum/OVA & OVA/OVA & alum/OVA & OVA/OVA \\
\hline MACS & $36.1 \pm 4.9$ & $32.2 \pm 4.8$ & $36.4 \pm 4.1$ & $49.1 \pm 13.2$ \\
\hline EOS & $0.2 \pm 0.1$ & ${ }^{*} 150.2 \pm 30.7$ & $0.2 \pm 0.1$ & ${ }^{*} 213.8 \pm 27.9$ \\
\hline PMN & $1.6 \pm 0.6$ & $* 21.0 \pm 9.3$ & $0.5 \pm 0.2$ & ${ }^{*} 21.4 \pm 8.0$ \\
\hline LYMPH & $0.9 \pm 0.2$ & ${ }^{*} 6.0 \pm 1.4$ & $0.4 \pm 0.2$ & $* 13.5 \pm 5.2$ \\
\hline 6 Chall. $+7 d$ & \multicolumn{2}{|c|}{ Balb/C } & \multicolumn{2}{|c|}{ Glrx1\% } \\
\hline $10^{3}$ & alum/OVA & OVA/OVA & alum/OVA & OVA/OVA \\
\hline MACS & $49.1 \pm 11.1$ & $43.7 \pm 7.2$ & $50.3 \pm 5.6$ & $44.3 \pm 7.2$ \\
\hline EOS & $06 \pm 0.3$ & ${ }^{*} 169.1 \pm 21.5$ & $0.1 \pm 0.0$ & $* 133.0 \pm 38.0$ \\
\hline PMN & $1.2 \pm 0.5$ & $1.3 \pm 0.7$ & $3.5 \pm 1.5$ & $1.1 \pm 0.4$ \\
\hline LYMPH & $1.3 \pm 0.7$ & ${ }^{*} 6.7 \pm 2.5$ & $10.7 \pm 5.4$ & ${ }^{*} 4.9 \pm 3.9$ \\
\hline
\end{tabular}

Table 1. Inflammatory cell profiles in BAL following OVA sensitization and challenge of WT or $\mathrm{Glrx} \mathbf{1}^{\%}$ mice. A minimum of 300 cells were enumerated by two independent investigators. Results were averaged and are expressed as means \pm SEM from 8 to 10 mice per group. ${ }^{*} P<0.05$ (ANOVA) compared with respective alum/OVA control groups. ${ }^{\dagger} P<0.05$ (ANOVA) compared with WT OVA/OVA groups. 
OVAlbumin-induced expression of pro-inflammatory mediators is reduced $48 \mathrm{hr}$ following 3 challenges in Glrx $1^{-}$mice. We evaluated the expression of pro-inflammatory mediators by assessment of mRNA in whole-lung homogenates, as well as overall content in BAL fluid. Results in Table 2 demonstrate significant increases in mRNA expression of KC, IL-6, RANTES, CCL-20, and IL-5 in WT mice $48 \mathrm{hr}$ after 3 or 6 OVA challenges, which tended to decrease 7 days following cessation of the 6 challenge regimen. The content of CCL-20 and KC was increased in BAL in mice immunized and challenged with OVA at all examined time points (Table 3), while BAL levels of RANTES and IL-6 remained widely unchanged. Fortyeight $\mathrm{hr}$ after 3 OVA challenges, G/rx $1^{\%}$ mice exhibited significant decreases in mRNA expression of KC and CCL-20 in lung tissue (Table 2, top), with accompanying decreases of these chemokines in the BAL (Table 3, top), compared to WT mice. Although small increases in mRNA expression of IL-4 and IL-13 were detected in lung tissue in response to sensitization and challenge with OVA, no clear differences were detected between WT and GIrx $1^{\%}$ mice (data not shown). Collectively, these findings suggest that ablation of the GIrx1 gene tended to decrease expression of pro-inflammatory mediators, but did not have a strong impact on overall allergic inflammation, in particular at the later time points that were investigated herein.

\begin{tabular}{|c|c|c|c|c|}
\hline 3 Chall. $+48 \mathrm{hr}$ & \multicolumn{2}{|c|}{ Balb/C } & \multicolumn{2}{|c|}{ G/rx $1 \%$} \\
\hline $\begin{array}{c}\text { Relative } \\
\text { Expression }\end{array}$ & alum/OVA & OVA/OVA & alum/OVA & OVA/OVA \\
\hline CCL-20 & $1.0 \pm 0.3$ & ${ }^{*} 3.8 \pm 0.5$ & $1.4 \pm 0.2$ & $\dagger 1.9 \pm 0.3$ \\
\hline IL-6 & $1.0 \pm 0.2$ & $1.5 \pm 0.4$ & $0.4 \pm 0.1$ & $1.4 \pm 0.3$ \\
\hline RANTES & $1.0 \pm 0.1$ & $0.8 \pm 0.1$ & $1.0 \pm 0.2$ & $1.0 \pm 0.1$ \\
\hline KC & $1.0 \pm 0.1$ & ${ }^{*} 7.6 \pm 1.2$ & $0.9 \pm 02$ & $\dagger 3.6 \pm 0.9$ \\
\hline IL-5 & $10 \pm 0.3$ & ${ }^{*} 9.4 \pm 28$ & $0.9 \pm 0.4$ & $+1.1 \pm 0.5$ \\
\hline 6 Chall. $+48 \mathrm{hr}$ & \multicolumn{2}{|c|}{ Balb/C } & \multicolumn{2}{|c|}{ G/rx $1 \%$} \\
\hline $\begin{array}{c}\text { Relative } \\
\text { Expression }\end{array}$ & alum/OVA & OVA/OVA & alum/OVA & OVA/OVA \\
\hline CCL-20 & $1.0 \pm 0.3$ & $\star 2.4 \pm 0.7$ & $0.6 \pm 0.1$ & $2.4 \pm 0.6$ \\
\hline IL-6 & $10 \pm 0.1$ & ${ }^{*} 4.8 \pm 1.1$ & $0.6 \pm 0.2$ & $2.1 \pm 0.7$ \\
\hline RANTES & $10 \pm 02$ & ${ }^{*} 9.3 \pm 2.2$ & $1.7 \pm 0.5$ & $4.1 \pm 0.7$ \\
\hline KC & $10 \pm 02$ & $2.4 \pm 12$ & $2.1 \pm 0.8$ & $1.3 \pm 0.5$ \\
\hline IL-5 & $1.0 \pm 0.1$ & ${ }^{*} 6.2 \pm 1.5$ & $2.1 \pm 1.6$ & $9.8 \pm 2.0$ \\
\hline
\end{tabular}

Table 2. Analysis of mRNA expression of NF-KB-dependent inflammatory cytokines in lung homogenates from WT and GIrx $1^{-1}$ mice subjected to sensitization and challenge with OVA. CCL-20, IL-6, RANTES, KC, and IL-5 mRNA from whole lung homogenates of OVA/OVA or alum/OVA mice were analyzed by real time PCR analyses. Results were normalized to the housekeeping gene cyclophilin. Data are expressed as fold increases in expression compared with WT alum/OVA controls. ${ }^{*} P<0.05$ (ANOVA) compared with respective alum/OVA control groups. ${ }^{\dagger} P$ $<0.05$ (ANOVA) compared with WT OVA/OVA groups. 


\begin{tabular}{|c|c|c|c|c|}
\hline 3 Chall. $+48 \mathrm{hr}$ & \multicolumn{2}{|c|}{ Balb/C } & \multicolumn{2}{|c|}{$\underline{G / n \times 1^{\%}}$} \\
\hline $\mathrm{pg} / \mathrm{ml}$ & alum/OVA & OVA/OVA & alum/OVA & OVA/OVA \\
\hline CCL-20 & $94.6 \pm 26.6$ & ${ }^{*} 183.6 \pm 22.9$ & $55.7 \pm 9.8$ & $\dagger 83.3 \pm 14.3$ \\
\hline IL-6 & $2.0 \pm 0.3$ & $2.7 \pm 0.4$ & $2.0 \pm 0.4$ & ${ }^{*} 6.1 \pm 1.1$ \\
\hline RANTES & $16.5 \pm 1.5$ & $21.2 \pm 4.0$ & $17.2 \pm 2.5$ & $20.4 \pm 3.7$ \\
\hline KC & $44.0 \pm 5.6$ & ${ }^{*} 249.7 \pm 15.9$ & $29.1 \pm 4.6$ & ${ }^{*}+161.7 \pm 27.9$ \\
\hline 6 Chall. $+48 \mathrm{hr}$ & \multicolumn{2}{|c|}{ Balb/C } & \multicolumn{2}{|r|}{$\underline{\text { GIrx } \times 1 \%}$} \\
\hline $\mathrm{pg} / \mathrm{ml}$ & alum/OVA & OVA/OVA & alum/OVA & OVAIOVA \\
\hline CCL-20 & $40.3 \pm 16,4$ & $* 95.7 \pm 31.8$ & $20.2 \pm 5.9$ & ${ }^{*} 50.1 \pm 16.8$ \\
\hline IL-6 & $16.8 \pm 3.9$ & $16.2 \pm 2.5$ & $16.1 \pm 3.9$ & $14.2 \pm 2.0$ \\
\hline RANTES & $79.7 \pm 19.9$ & $67.8 \pm 8.5$ & $76.3 \pm 22.5$ & $63.8 \pm 8.0$ \\
\hline KC & $1420 \pm 21.3$ & ${ }^{*} 677.9 \pm 147.2$ & $128.6 \pm 30.5$ & ${ }^{*} 478.7 \pm 104.3$ \\
\hline 6 Chall. $+7 d$ & \multicolumn{2}{|c|}{ Balb/C } & \multicolumn{2}{|c|}{$\underline{G l n} \times 1^{-1}$} \\
\hline $\mathrm{pg} / \mathrm{ml}$ & alum/OVA & OVAIOVA & alum/OVA & OVAIOVA \\
\hline CCL-20 & $60.2 \pm 19.3$ & ${ }^{*} 131.2 \pm 47.8$ & $86.2 \pm 13.3$ & $40.7 \pm 32.2$ \\
\hline IL-6 & $19.4 \pm 3.7$ & $18.2 \pm 4.4$ & $17.9 \pm 4.5$ & $10.5 \pm 1.7$ \\
\hline RANTES & $111.1 \pm 16.6$ & $69.9 \pm 12.5$ & $82.2 \pm 14.2$ & $43.0 \pm 9.5$ \\
\hline KC & $227.9 \pm 80.5$ & ${ }^{*} 667.3 \pm 164.0$ & $110.4 \pm 198$ & $502,0 \pm 116.9$ \\
\hline
\end{tabular}

Table 3. Analysis of content of NF-kB-dependent inflammatory cytokines in BAL from WT and Glrx ${ }^{\%}$ mice subjected to sensitization and challenge with OVA. BAL fluid was analyzed by ELISA for cytokines: CCL-20, IL-6, RANTES, and KC. Data are expressed as means \pm SEM from 8 to 10 mice per group. ${ }^{*} P<0.05$ (ANOVA) compared with respective alum/OVA control groups. ${ }^{+} P<0.05$ (ANOVA) compared with WT OVA/OVA groups.

Ablation of GIrx1 causes enhanced resolution of Ovalbumin-induced AHR and mucus metaplasia. To address the functional consequences of the lack of Glrx1, a forced oscillation technique was used to evaluate alterations in respiratory mechanics in response to OVA sensitization and challenge. As expected, in response to OVA sensitization and challenge, airway resistance $\left(R_{N}\right)$ (Fig. 3A), tissue dampening (G) (Fig. 3B), and elastance/stiffness (H) (Fig. 3C) were significantly increased in WT animals, with the most prominent increases occurring $48 \mathrm{hr}$ post 3 challenges with OVA. It is worth noting that one week post cessation of OVA challenges, all parameters of AHR remained significantly increased in WT animals. $48 \mathrm{hr}$ after 3 challenges, G/r $\times 1^{\%}$ mice demonstrated comparable increases over respective controls in $R_{N}$ and $G$ at 12.5 and $25 \mathrm{mg} / \mathrm{ml}$ of Mch. Although tissue elastance $(H)$ tended to increase in Glrx $1^{\%}$ mice in response to sensitization and challenge with OVA, compared with alum/OVA controls, these increases did not reach statistical significance. Glr $\times 1^{\%}$ mice showed significant attenuation in tissue dampening (G) $48 \mathrm{hr}$ after 6 challenges with OVA, compared to WT controls. Importantly, 
parameters of AHR were significantly decreased in $\mathrm{Glrx}^{\%}$ mice compared to WT groups 7 days post cessation of the 6 day challenge regimen (Fig. 3A-C, bottom).

A. $\mathrm{Rn}$

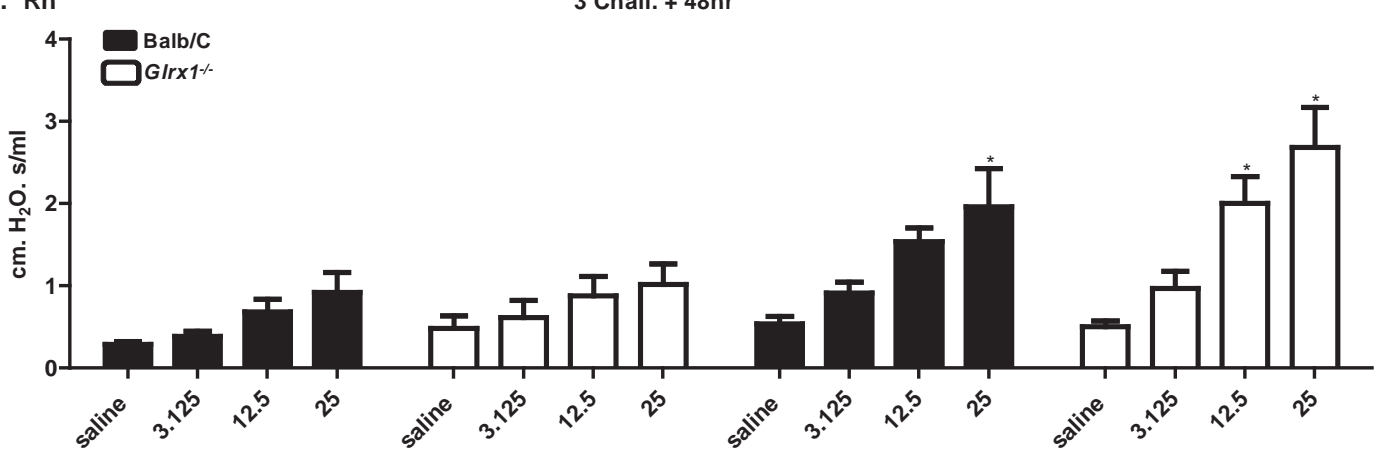

alum/OVA

6 Chall. $+48 \mathrm{hr}$

OVA/OVA

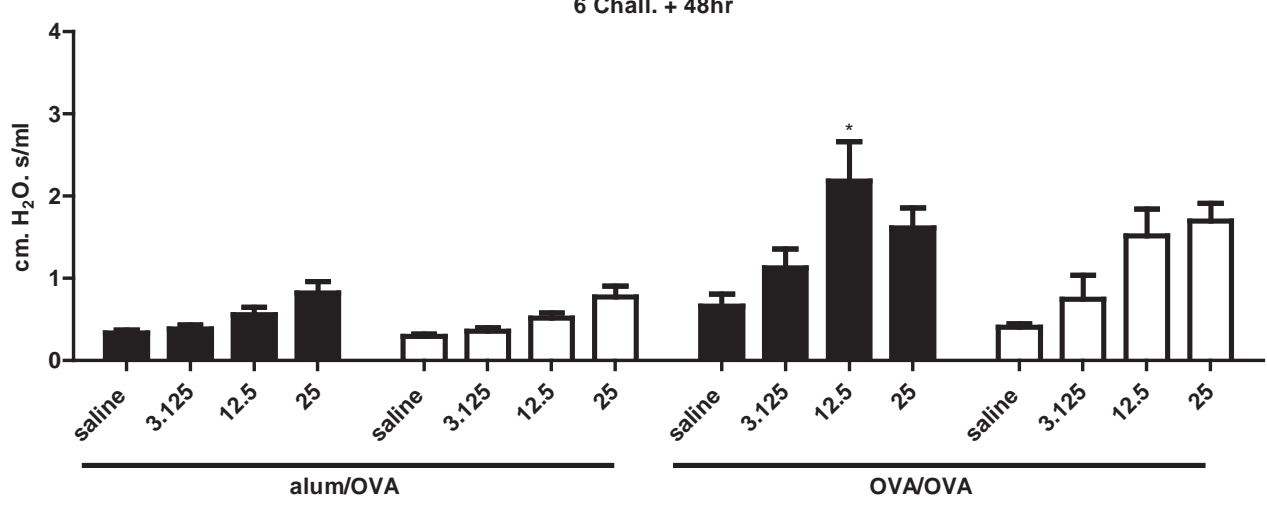

6 Chall. $+7 d$

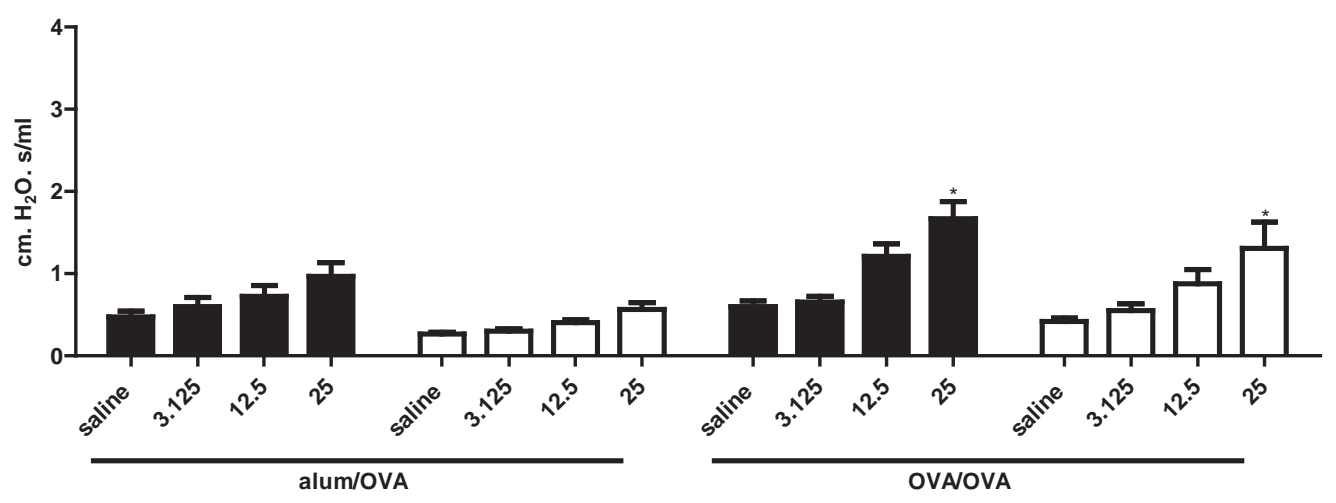


B. $\mathbf{G}$

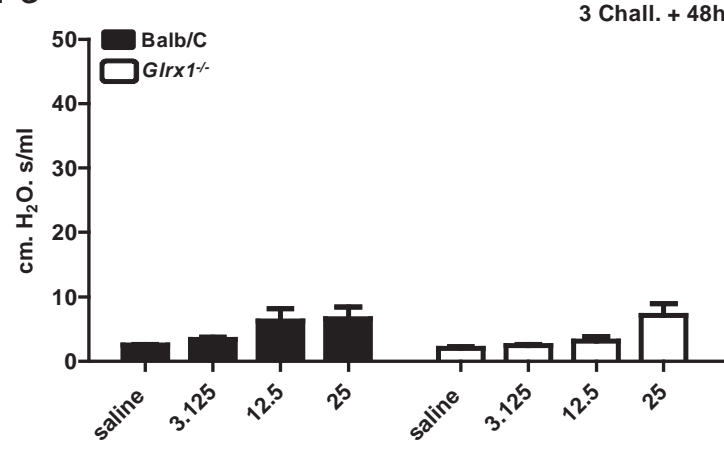

alum/OVA
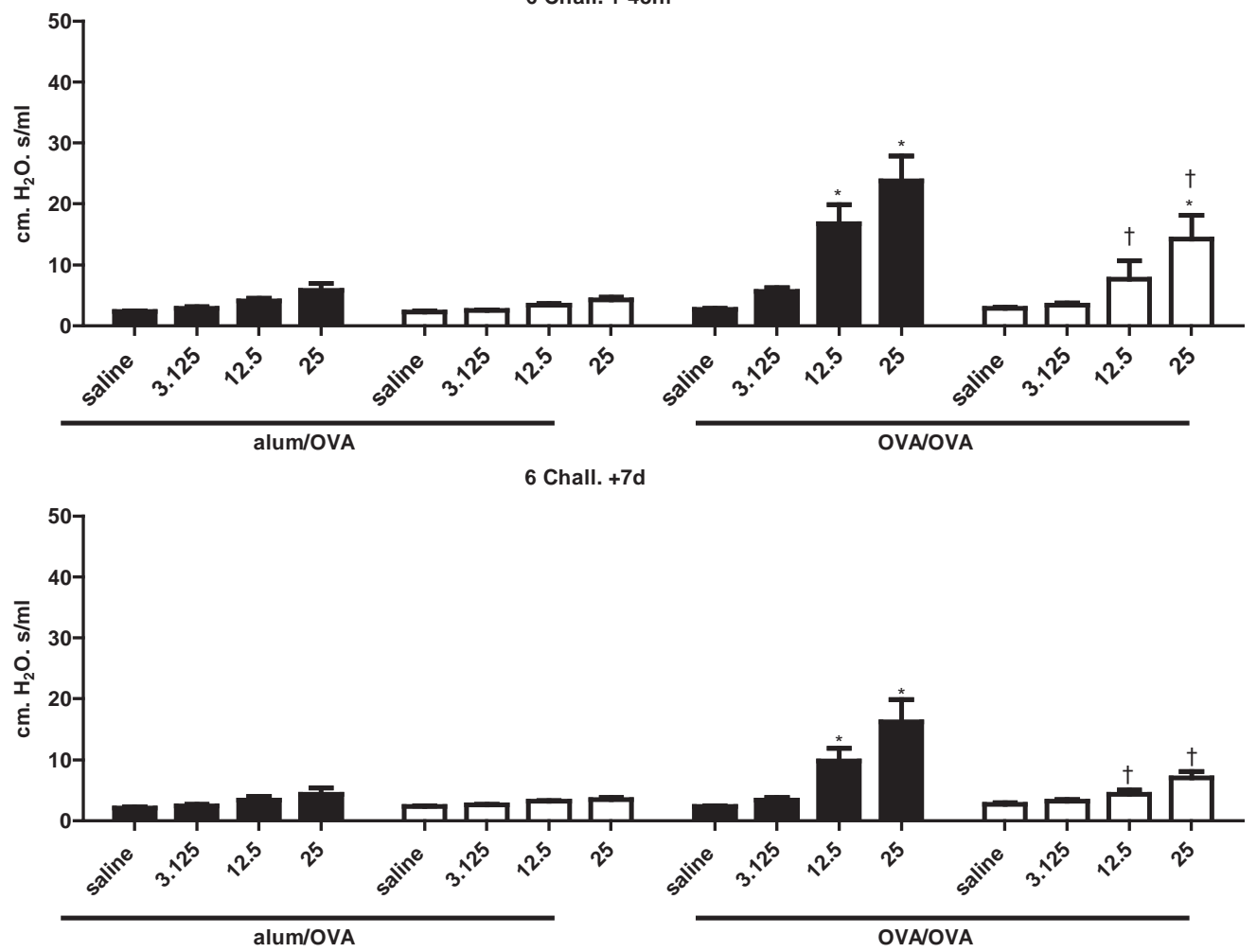
C. $\mathrm{H}$
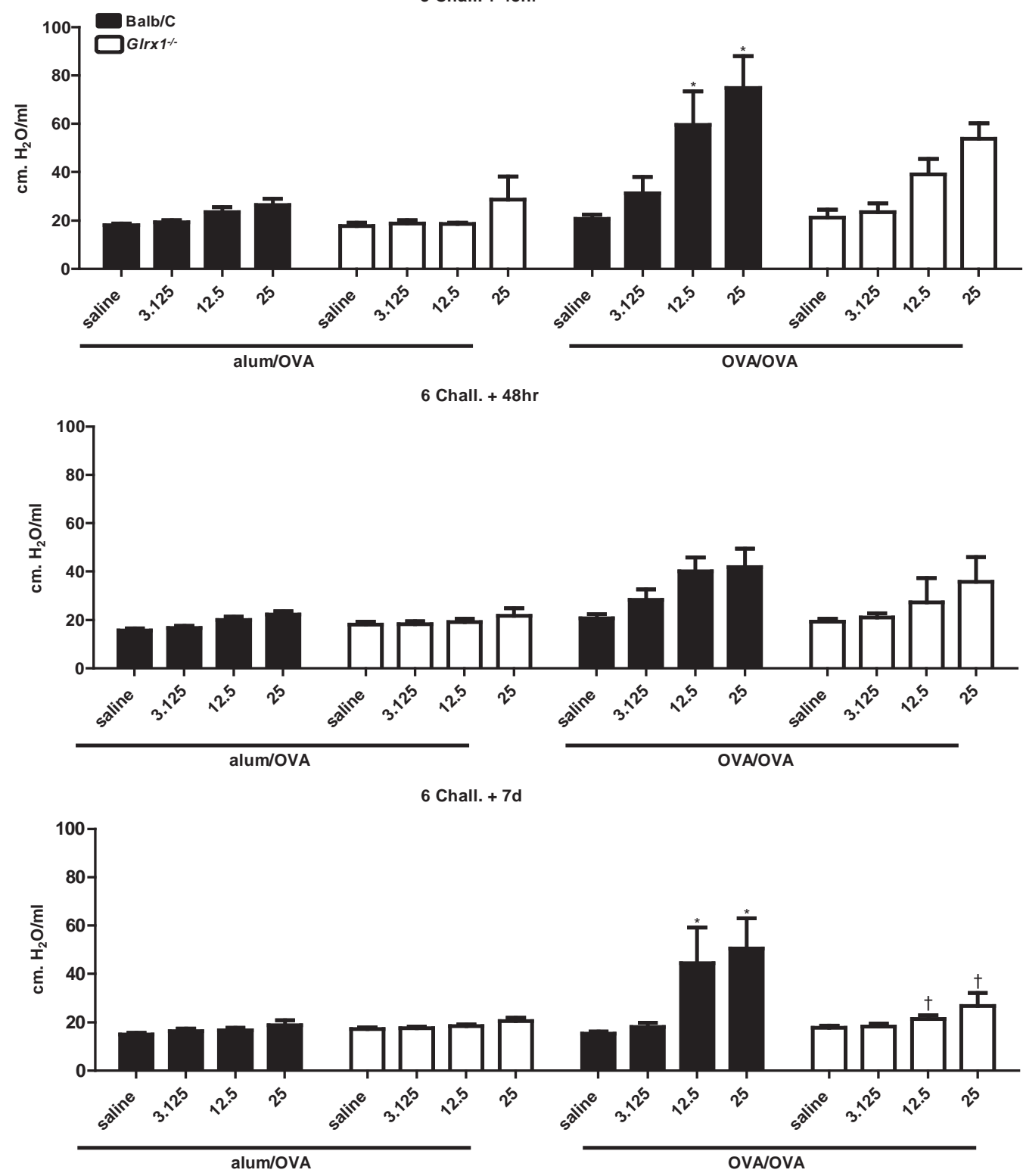

Figure 3. Assessment of airway hyperresponsiveness in WT or GIrx ${ }^{\%}$ mice following sensitization and challenge with OVA. Changes in respiratory mechanics were analyzed in mock-sensitized (alum/OVA) or OVA-sensitized (OVA/OVA) WT (black bars) or G/rx $1^{-1-}$ mice (white bars) $48 \mathrm{hr}$ following 3 or 6 challenges with aerosolized OVA, or 7 days following the sixth daily aerosolized OVA challenge. Ascending doses of methacholine were administered to determine (A) $R_{N}$ (airway resistance), (B) G (tissue dampening), and (C) $H$ (elastance/stiffness) parameters. Data are expressed as the percent change from baseline measurements ( \pm SEM) for each of the parameters measured from 8 to 10 mice per group. ${ }^{*} P<0.05$ (ANOVA) compared with respective alum/OVA control groups. ${ }^{t} P<0.05$ (ANOVA) compared with WT OVA/OVA groups. 
Defining features of asthma include excess mucus secretion, goblet cell hyperplasia and sub-mucosal gland hypertrophy [17, 20], which have been shown to contribute to airway closure and hyperresponsiveness [21, 22]. mRNA analysis of the mucus genes MUC5ac and GOB5, revealed similar increases in expression in response to OVA in WT and $G / r \times 1^{-1-}$ mice $48 \mathrm{hr}$ after 6 challenges with OVA (Fig. 4A, 4B). Decreases in MUC5ac mRNA were apparent in Glrx $1^{\%}$ compared to WT mice 7 days post the 6 day OVA challenge regimen (Fig. 4A).

A. MUC5ac
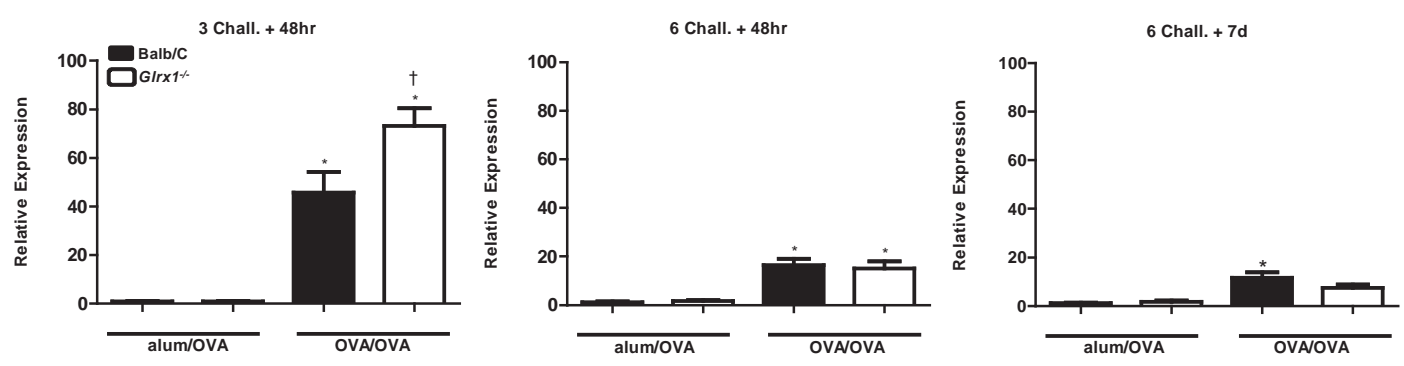

B. GOB5
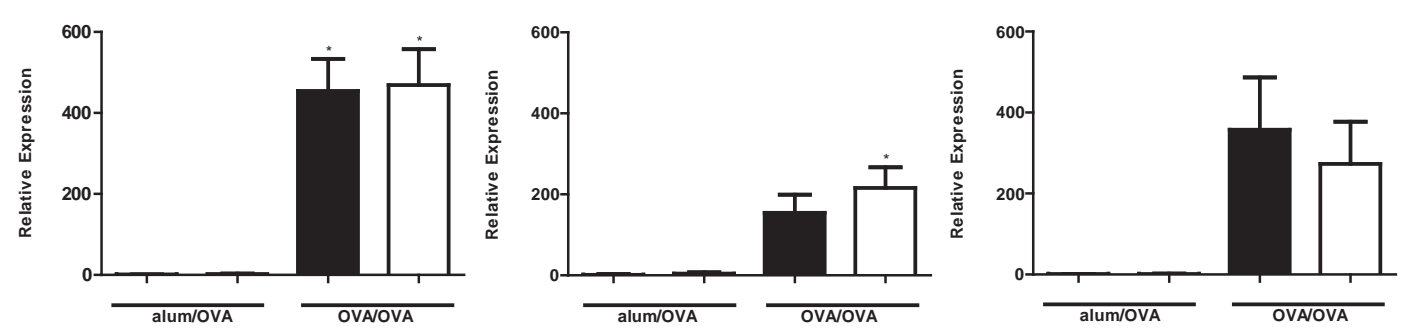

Figure 4. Evaluation of GOB5, MUC5ac, and IL-13 gene expression in WT and GIrx $1^{\%}$ mice subjected to sensitization and challenge with OVA. RNA from lung homogenates of OVA-sensitized and challenged mice (OVA/OVA) or mock-sensitized mice (alum/OVA) was analyzed by real time PCR, and results were normalized to the housekeeping gene cyclophilin. Data are expressed as fold increases in expression compared with WT alum/OVA controls. ${ }^{*} P<0.05$ (ANOVA) compared with respective alum/OVA control groups. ${ }^{\dagger} P<0.05$ (ANOVA) compared with WT OVA/OVA groups. Black bars: BALB/c mice, white bars: $G / r \times 1^{-1-}$ mice.

IL-13 levels in BAL fluid recovered from WT mice sensitized and challenged with OVA, were significantly elevated over alum/OVA controls in WT mice $48 \mathrm{hr}$ post 3 challenges (Fig. 5A). Although no clear increases in IL-13 content in the BAL occurred in OVA sensitized and challenged mice compared to the alum/OVA group, at the later time points, IL-13 content in BAL from Glrx $1^{\%}$ mice was slightly decreased $48 \mathrm{hr}$ post 6 challenges, and significantly decreased after 7 days compared to WT groups (Fig. $5 \mathrm{~A}$, middle and right). Consistent with these observations, histopathological analysis of lungs from WT and $\mathrm{G} / \mathrm{r} \times \mathrm{1}^{-/}$mice revealed comparable mucus metaplasia $48 \mathrm{hr}$ after 3 and 6 aerosolized OVA challenges 
(Fig. 5B). Although significant increases in mucus metaplasia remained 7 days following cessation of 6 challenges in WT mice, PAS positivity was significantly reduced in Glr $\times 1^{-1}$ mice (Fig. 5B, right, Fig. 5C). In aggregate, these findings demonstrate that $\mathrm{Glr} \times 1^{\%}$ mice display enhanced resolution of AHR and mucus metaplasia, compared with WT counterparts.

A. IL-13
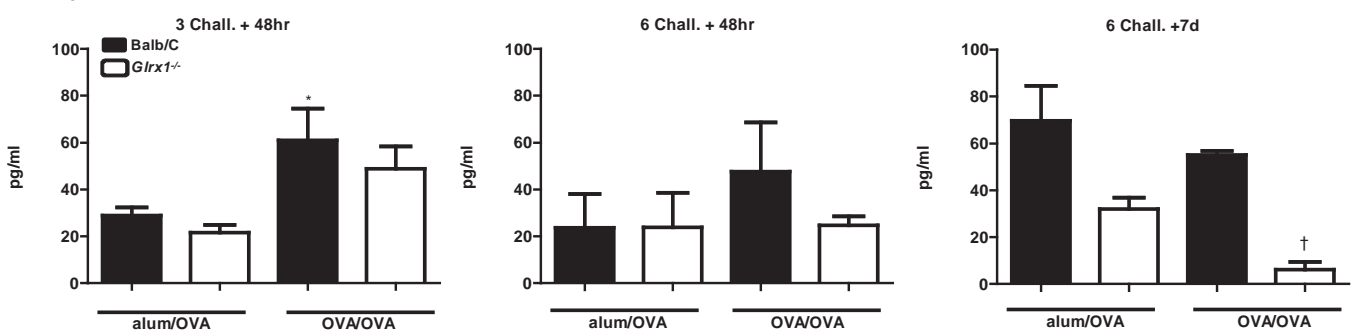

B. PAS
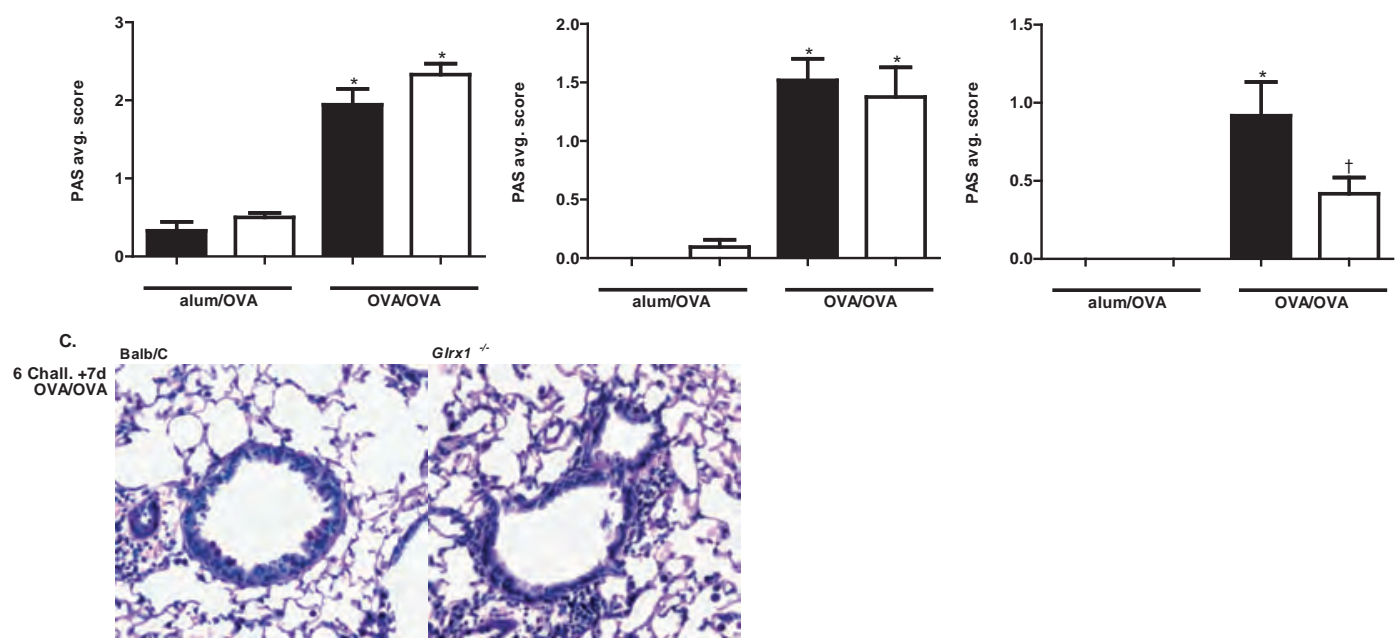

Figure 5. Evaluation of IL-13 content in BAL and mucus metaplasia in WT and GIrx $1 \%$ mice subjected to sensitization and challenge with OVA. (A) IL-13 content in the BAL was determined by ELISA. Data are expressed as means \pm SEM from 8 to 10 mice per group. ${ }^{*} P<0.05$ (ANOVA) compared with respective alum/OVA control groups. ${ }^{+} P<0.05$ (ANOVA) compared with WT OVA/OVA groups. (B) Mucus metaplasia was assessed in periodic acid-Schiff-stained bronchiolar epithelium from WT and Glrx $1^{\%}$ mice sensitized and challenged with OVA (OVA/OVA). Mucus metaplasia was evaluated by obtaining three images of small bronchioles (20X objective) of similar dimensions from each mouse. Average scores of two independent evaluations were determined. Data are expressed as means \pm SEM from 8 to 10 mice per group. ${ }^{*} P<0.05$ (Kruskal-Wallis) compared with respective alum/OVA control groups. ${ }^{\dagger} P<0.05$ (Kruskal-Wallis) compared with WT OVA/OVA groups. Black bars: BALB/c mice, white bars: $G / r \times 1^{\%-}$ mice.

Absence of glutaredoxin-1 leads to increases in protein S-glutathionylation in response to sensitization and challenge with OVA. Under physiological conditions, the main function of glutaredoxin-1 is to de- 
glutathionylate proteins restoring protein sulfhydryl groups. In response to sensitization and challenge with OVA, increases in endogenous Glrx1 content occurred in WT mice over time (Fig. 6A). In WT mice, no clear increases in overall PSSG content occurred in response to OVA, compared with alum/OVA controls at any of the time points investigated. We next assessed whether alterations in PSSG content occurred in lung tissue from mice lacking Glrx1 compared to WT animals. Ablation of Glrx1 resulted in slight increases in PSSG content, in the alum/OVA group compared to WT mice. Slight, but significant increases in PSSG content were apparent $48 \mathrm{hr}$ after 3 challenges in $\mathrm{Glr} \times 1^{\%}$ mice, with striking increases occurring $48 \mathrm{hr}$ after 6 challenges (Fig. 6B). Surprisingly, increases in PSSG were no longer apparent in Glrx $1^{\%-}$ mice, 1 week post cessation of OVA challenges.

A.

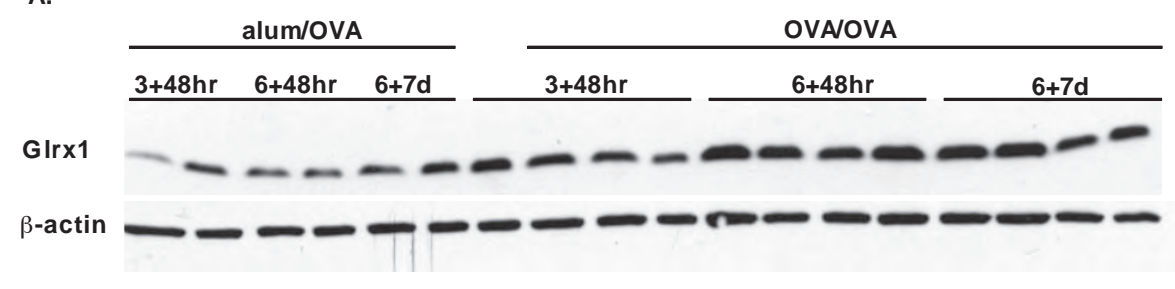

B.

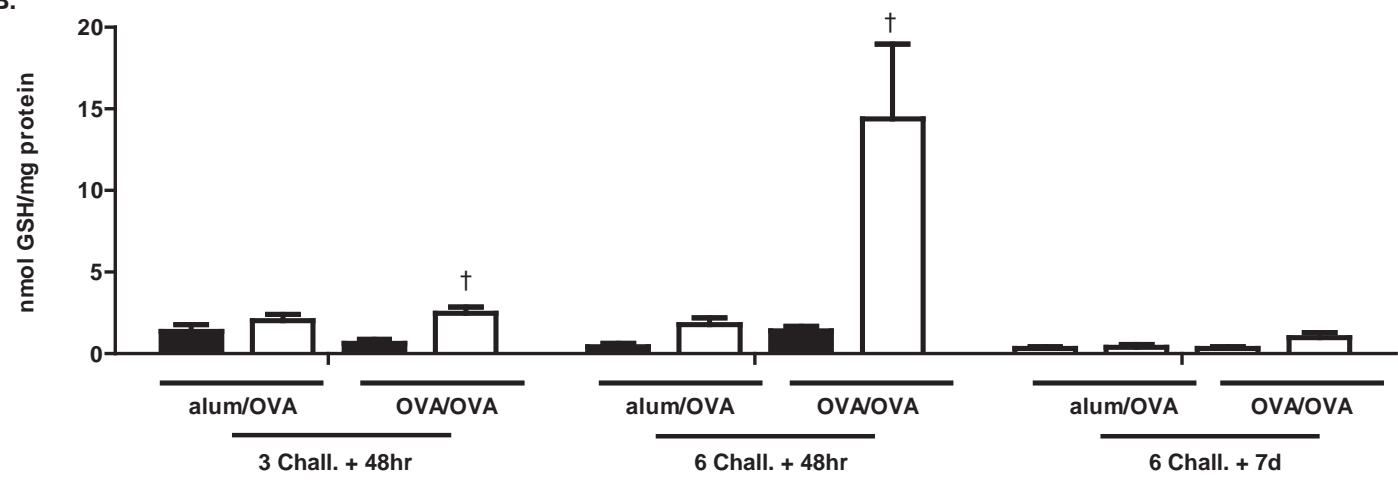

Figure 6. Assessment of protein $S$-glutathionylation and Glrx1 expression in WT and Glrx $1^{-/}$mice following sensitization and challenge with OVA. (A) Assessment of GIrx1 content in lung tissue from WT mice at different time points following sensitization and challenge with OVA. Lung tissues were homogenized, and GIrx1 content assessed in via Western Blot analyses/ $\beta$-actin is shown as a loading control. Results from two independent mice are shown per time point in the Alum/OVA groups, whereas 4 independent mice were analyzed per time point in the OVA/OVA groups. (B) Biochemical analysis of protein S-glutathionylation in homogenized lung tissues of WT (black bars) and Glrx $1^{-}$mice (white bars) $48 \mathrm{hr}$ and 7 days following the sixth daily aerosolized OVA challenge. Homogenized lung tissue was prepared, proteins were precipitated, and glutathione was released using Naborohydride and quantified using the glutathione disulfide (GSSG) reductase recycling assay, with 5,5'-dithiobis-(2nitrobenzoic acid) (DTNB) as substrate. Na-borohydride-dependent formation of 5'-thio-2-nitrobenzoic acid was calculated and normalized to protein content. Data are expressed as nmol tripeptide glutathione (GSH)/mg protein. Data are expressed as means \pm SEM from 8 to 10 mice per group. ${ }^{*} P<0.05$ (ANOVA) compared with respective alum/OVA control groups. ${ }^{\dagger} P<0.05$ (ANOVA) compared with WT OVA/OVA groups. 


\section{DISCUSSION}

PSSG has emerged as an archetypal, redox-dependent post-translational modification of target protein cysteines. PSSG not only has the potential to affect protein structure and function, it also protects proteins from irreversible thiol oxidations. The impact of protein S-glutathionylation depends upon the target protein, and activation [23, 24], gain of function [25], and inhibition of various proteins [12, 26, 27] has been observed [8]. The biological significance of PSSG is bolstered by the evolution of glutaredoxins, thioltransferases that catalyse reversible $S$-glutathionylation and de-glutathionylation reactions. Under physiological conditions, cytosolic Glrx1 preferentially catalyses deglutathionylation reactions via the $\mathrm{N}$-terminal active-site cysteine. This results in S-glutathionylation of Glrx1 itself, which is subsequently reduced by a second GSH molecule $[7,8]$. Patho-physiological functions for GIrx1 are emerging that include roles in the regulation of apoptosis, and transcription, tissue inflammation and remodeling [7].

In the current study, we addressed the impact of ablation of GIrx1 on OVAlbumin-induced allergic airways disease. Our results demonstrate clear increases in the content of PSSG reactivity in lung tissues from GIrx $1^{-1}$ mice after sensitization and repeated challenges with OVA, compared with WT mice, consistent with its role in catalysis of de-glutathionylation reactions in physiological settings. Surprisingly, 7 days after 6 challenges with antigen, PSSG content decreased in the lungs of G/rx $1 \%$ mice (Fig. 6B). A previous study reported no evidence for compensation of various antioxidant enzymes or GIrx2 content in the lungs of $\mathrm{G} / r \times 1^{\%}$ mice, which also lacked detectable de-glutathionylation activity [13]. However, our data indicate the presence of other de-glutathionylating events or enzymes in the lung in the setting of allergic inflammation. In this regard, putative roles for the related oxidoreductase, thioredoxin [28] as well as sulfiredoxin $[29,30]$ have been suggested, which could account for decreases in PSSG content over time in lungs from Glrx $1^{-/}$mice. Additional analyses that were beyond the scope of the present study will be required to determine the source of the "de-glulathionylating activity" in lungs of $\mathrm{G} / \mathrm{r} \times 1^{-/-}$mice. In the present study we also observed slight increases in PSSG in lung tissues from mock sensitized (alum/OVA) G/rx $1^{-}$mice compared WT alum/OVA groups, findings that are in apparent contrast to previous reports indicating that the systemic lack of GIrx1 does not affect baseline PSSG [11, 13]. It is plausible that aerosolized antigen, in the absence of previous sensitization, causes a mild perturbation of the redox status, which could be more apparent in the absence of Glrx1.

Our laboratory has previously demonstrated a causal role for activation of the transcription factor, NF-KB within the bronchial epithelium in orchestrating allergic inflammation [14, 18, 31]. The transcription factor, NF-KB has been shown to be inhibited via S-glutathionylation of IKK $\beta$, RelA and p50 
$[12,27,32,33]$. Genetic ablation or siRNA-mediated knock-down of Glrx1 resulted in enhanced Sglutathionylation of inhibitory kappa B kinase-beta, (IKK $\beta$ ), and attenuated production of proinflammatory mediators by epithelial cells following stimulation with LPS. Conversely, overexpression of Glrx1 prolonged LPS-induced pro-inflammatory responses in lung epithelial cells, in association with decreases in glutathionylated IKK $\beta$, and prolonged activation of NF-KB [12, 32]. It is therefore surprising that, despite increases in the overall content of PSSG in lung tissue, the overall extent of airway inflammation was only transiently diminished in $\mathrm{Glrx} \mathrm{1}^{-/}$mice after sensitization and challenge with OVA compared with WT mice. Nonetheless, expression of pro-inflammatory mediators was consistently decreased in Glrx1\% mice compared with WT animals. It is possible that Glrx1 affects the resolution of airway inflammation. Since inflammatory cells in the airways are still prominent 7 days after cessation of the 6 challenge regimen, more extended time points will be required to determine the impact of GIrx1 on the resolution of allergic disease. Within the time frame of investigation of the present study we failed to demonstrate clear differences in nuclear content of RelA in lung homogenates from WT and Glrx $1^{-1-}$ mice (data not shown). Future studies will be required to elucidate whether $S$-glutathionylation of NF-KB family members within the lung epithelium are affected in Glrx $1^{-1}$ mice. Such analyses were beyond the scope of the present study, and will require dissection of bronchiolar epithelium, the compartment wherein NF-KB activation is predominant. It is also plausible that the systemic ablation of Glrx1 may have obscured the contribution of Glrx1 specifically in airway epithelium towards NF-KB activation, and subsequent inflammatory responses. Therefore, elucidation of the exact role of GIrx1 in orchestrating inflammation awaits additional studies aimed at conditional manipulation of Glrx1 during specific times, and specifically within the bronchial epithelium during allergen-driven inflammation.

One of the most striking phenotypes of $\mathrm{G} / \mathrm{r} \times 1^{\%}$ mice in the present study was the enhanced resolution of AHR, compared with WT mice. Despite the sustained presence of OVA-induced inflammatory cells in the lungs at the time of assessment of respiratory mechanics, G/rx $1^{\%}$ animals exhibited marked reductions in hyperresponsiveness to methacholine in comparison with WT mice. Notably, the elastance $(\mathrm{H})$ and tissue dampening $(\mathrm{G})$ parameters in $\mathrm{G} / \mathrm{r} \times 1^{\%}$ mice sensitized to OVA were significantly reduced 7 days following OVA challenge, whereas increases in Newtonian resistance $\left(R_{N}\right)$, reflective of conducting airways, still occurred in $\mathrm{Glrx}^{-/-}$mice, albeit to a slightly lesser extent than WT mice. These findings suggest a more pronounced impact of Glrx1 deficiency in the lung periphery. Additionally, Glrx $1 \%$ mice demonstrated significant decreases in gene expression of MUC5ac and mucus metaplasia 7 days post cessation of OVA challenges, compared to WT mice (Fig. 4 and Fig. 5). These findings suggest that the enhanced resolution of $A H R$ in $G / r \times 1^{-1}$ mice may be functionally linked to 
altered regulation of mucin gene expression, mucin glycoprotein processing, and/or secretion. A firm link between oxidants and mucus metaplasia has been established; oxidants can increase expression of Muc5AC mRNA [34-38] and a role for oxidants in the transcriptional upregulation of the IL-13 gene has been shown [39]. In keratinocytes stimulated with IL-4 and IL-13, activation of dual specificity oxidase-1 was reported to be critical in the activation of STAT6, following oxidative inactivation of protein tyrosine phosphatase 1B. These collective studies demonstrate that changes in redox homeostasis are important in the development of mucus metaplasia. However, it is unknown to date whether the signals that regulate mucin gene expression, or intracellular processing of mucins is regulated via GIrx1-controlled Sglutathionylation.

S-glutathionylation reflects an oxidative event that can be triggered via multiple biochemical mechanisms, including oxidant production via activation of NADPH oxidases $[7,8]$. Oxidative events have been strongly implicated in the pathogenesis of allergic airways disease $[5,40,41]$. It is therefore perhaps counter-intuitive that in mice lacking GIrx1 wherein PSSG (an oxidative event) is increased, AHR, mucus metaplasia, and expression of pro-inflammatory mediators are decreased. These findings suggest putative beneficial function for S-glutathionylation in the pathophysiology of allergic airways disease. The exact mechanisms whereby S-glutathionylation exerts protective effects during the pathogenesis of allergic airways disease awaits additional investigation into the proteins that are targeted via $S$ glutathionylation, and the impact of S-glutathionylation on the regulation of their structure and function. It is plausible that $S$-glutathionylation exerts its protective function by preventing irreversible protein oxidations. In this regard, irreversible protein oxidation has been detected in lungs from mice exposed to cigarette smoke in association with loss of S-glutathionylation [42].

In conclusion, the present study demonstrates that a systemic loss of Glrx1 attenuates airways hyperresponsiveness, in association with decreased mucus metaplasia in antigen-induced allergic airways disease. Our results highlight the potential functional importance of the GIrx1/PSSG redox module in the resolution of airway hyperresponsiveness in allergic asthma, findings that could hold clinical relevance and offer potential therapeutic opportunities. 


\section{REFERENCES}

1. Andreadis, A.A., et al., Oxidative and nitrosative events in asthma. Free Radic Biol Med, 2003. 35(3): p. 213-25.

2. Comhair, S.A., et al., Correlation of systemic superoxide dismutase deficiency to airflow obstruction in asthma. Am J Respir Crit Care Med, 2005. 172(3): p. 306-13.

3. Comhair, S.A., et al., Superoxide dismutase inactivation in pathophysiology of asthmatic airway remodeling and reactivity. Am J Pathol, 2005. 166(3): p. 663-74.

4. Fitzpatrick, A.M., et al., Airway glutathione homeostasis is altered in children with severe asthma: evidence for oxidant stress. J Allergy Clin Immunol, 2009. 123(1): p. 146-152 e8.

5. Reynaert, N.L., Glutathione biochemistry in asthma. Biochim Biophys Acta, 2011. 1810(11): p. 1045-51.

6. Rahman, I., et al., Glutathione, stress responses, and redox signaling in lung inflammation. Antioxid Redox Signal, 2005. 7(1-2): p. 42-59.

7. Mieyal, J.J., et al., Molecular mechanisms and clinical implications of reversible protein Sglutathionylation. Antioxid Redox Signal, 2008. 10(11): p. 1941-88.

8. Janssen-Heininger, Y.M., et al., Redox-based regulation of signal transduction: principles, pitfalls, and promises. Free Radic Biol Med, 2008. 45(1): p. 1-17.

9. Lillig, C.H., C. Berndt, and A. Holmgren, Glutaredoxin systems. Biochim Biophys Acta, 2008. 1780(11): p. 1304-17.

10. Reynaert, N.L., E.F. Wouters, and Y.M. Janssen-Heininger, Modulation of glutaredoxin-1 expression in a mouse model of allergic airway disease. Am J Respir Cell Mol Biol, 2007. 36(2): p. 147-51.

11. Aesif, S.W., et al., Ablation of glutaredoxin-1 attenuates lipopolysaccharide-induced lung inflammation and alveolar macrophage activation. Am J Respir Cell Mol Biol, 2011. 44(4): p. 4919.

12. Reynaert, N.L., et al., Dynamic redox control of NF-kappaB through glutaredoxin-regulated Sglutathionylation of inhibitory kappaB kinase beta. Proc Natl Acad Sci U S A, 2006. 103(35): p. 13086-91.

13. Ho, Y.S., et al., Targeted disruption of the glutaredoxin 1 gene does not sensitize adult mice to tissue injury induced by ischemia/reperfusion and hyperoxia. Free Radic Biol Med, 2007. 43(9): p. 1299-312.

14. Pantano, C., et al., Nuclear factor-kappaB activation in airway epithelium induces inflammation and hyperresponsiveness. Am J Respir Crit Care Med, 2008. 177(9): p. 959-69.

15. Lloyd, C.M. and S. Saglani, Asthma and allergy: the emerging epithelium. Nat Med, 2010. 16(3): p. 273-4.

16. Poynter, M.E., C.G. Irvin, and Y.M. Janssen-Heininger, Rapid activation of nuclear factor-kappaB in airway epithelium in a murine model of allergic airway inflammation. Am J Pathol, 2002. 160(4): p. 1325-34.

17. Riesenfeld, E.P., et al., Inhaled salmeterol and/or fluticasone alters structure/function in a murine model of allergic airways disease. Respir Res, 2010. 11: p. 22.

18. Ather, J.L., et al., Airway epithelial NF-kappaB activation promotes allergic sensitization to an innocuous inhaled antigen. Am J Respir Cell Mol Biol, 2011. 44(5): p. 631-8.

19. Rahman, I., A. Kode, and S.K. Biswas, Assay for quantitative determination of glutathione and glutathione disulfide levels using enzymatic recycling method. Nat Protoc, 2006. 1(6): p. 3159-65.

20. Morcillo, E.J. and J. Cortijo, Mucus and MUC in asthma. Curr Opin Pulm Med, 2006. 12(1): p. 1-6.

21. Evans, C.M., et al., Mucus hypersecretion in asthma: causes and effects. Curr Opin Pulm Med, 2009. 15(1): p. 4-11. 
22. Singer, M., et al., A MARCKS-related peptide blocks mucus hypersecretion in a mouse model of asthma. Nat Med, 2004. 10(2): p. 193-6.

23. Adachi, T., et al., S-glutathiolation of Ras mediates redox-sensitive signaling by angiotensin II in vascular smooth muscle cells. J Biol Chem, 2004. 279(28): p. 29857-62.

24. Adachi, T., et al., S-Glutathiolation by peroxynitrite activates SERCA during arterial relaxation by nitric oxide. Nat Med, 2004. 10(11): p. 1200-7.

25. Anathy, V., et al., Redox amplification of apoptosis by caspase-dependent cleavage of glutaredoxin 1 and S-glutathionylation of Fas. J Cell Biol, 2009. 184(2): p. 241-52.

26. Barrett, W.C., et al., Regulation of PTP1B via glutathionylation of the active site cysteine 215. Biochemistry, 1999. 38(20): p. 6699-705.

27. Pineda-Molina, E., et al., Glutathionylation of the $p 50$ subunit of NF-kappaB: a mechanism for redox-induced inhibition of DNA binding. Biochemistry, 2001. 40(47): p. 14134-42.

28. Greetham, D., et al., Thioredoxins function as deglutathionylase enzymes in the yeast Saccharomyces cerevisiae. BMC Biochem, 2010. 11: p. 3.

29. Findlay, V.J., et al., A novel role for human sulfiredoxin in the reversal of glutathionylation. Cancer Res, 2006. 66(13): p. 6800-6.

30. Park, J.W., et al., Deglutathionylation of 2-Cys peroxiredoxin is specifically catalyzed by sulfiredoxin. J Biol Chem, 2009. 284(35): p. 23364-74.

31. Poynter, M.E., et al., NF-kappa B activation in airways modulates allergic inflammation but not hyperresponsiveness. J Immunol, 2004. 173(11): p. 7003-9.

32. Aesif, S.W., et al., Activation of the glutaredoxin-1 gene by nuclear factor kappaB enhances signaling. Free Radic Biol Med, 2011. 51(6): p. 1249-57.

33. Qanungo, S., et al., Glutathione supplementation potentiates hypoxic apoptosis by Sglutathionylation of p65-NFkappaB. J Biol Chem, 2007. 282(25): p. 18427-36.

34. Wright, D.T., et al., Oxidant stress stimulates mucin secretion and PLC in airway epithelium via a nitric oxide-dependent mechanism. Am J Physiol, 1996. 271(5 Pt 1): p. L854-61.

35. Jang, M.K., et al., The tyrosine phosphatase, SHP-1, is involved in bronchial mucin production during oxidative stress. Biochem Biophys Res Commun, 2010. 393(1): p. 137-43.

36. Kim, H.J., et al., Epicatechin gallate suppresses oxidative stress-induced MUC5AC overexpression by interaction with epidermal growth factor receptor. Am J Respir Cell Mol Biol, 2010. 43(3): p. 349-57.

37. Adler, K.B., W.J. Holden-Stauffer, and J.E. Repine, Oxygen metabolites stimulate release of highmolecular-weight glycoconjugates by cell and organ cultures of rodent respiratory epithelium via an arachidonic acid-dependent mechanism. J Clin Invest, 1990. 85(1): p. 75-85.

38. Fischer, B.M. and J.A. Voynow, Neutrophil elastase induces MUC5AC gene expression in airway epithelium via a pathway involving reactive oxygen species. Am J Respir Cell Mol Biol, 2002. 26(4): p. 447-52.

39. Bansal, G., et al., Oxidant signaling for interleukin-13 gene expression in lung smooth muscle cells. Free Radic Biol Med, 2012. 52(9): p. 1552-9.

40. Ckless, K., et al., Epithelial, dendritic, and CD4(+) T cell regulation of and by reactive oxygen and nitrogen species in allergic sensitization. Biochim Biophys Acta, 2011. 1810(11): p. 1025-34.

41. Peden, D.B., The role of oxidative stress and innate immunity in $\mathrm{O}(3)$ and endotoxin-induced human allergic airway disease. Immunol Rev, 2011. 242(1): p. 91-105.

42. Kuipers, I., et al., Smoke decreases reversible oxidations S-glutathionylation and S-nitrosylation in mice. Free Radic Res, 2012. 46(2): p. 164-73. 


\section{CHAPTER 5}

Ablation of glutaredoxin-1 modulates airway neutrophilia, IL-17A and airways hyperresponsiveness in house dust mite-induced allergic airways disease

Sidra M. Hoffman, David G. Chapman, Karolyn G. Lahue, James D. Nolin, David H. McMillan, Jane T. Jones, Minara Aliyeva, Nirav Daphtary, Jennifer L. Ather, Ye-Shih Ho, Vikas Anathy, Charles G. Irvin, Emiel F.M. Wouters, Matthew E. Poynter and Yvonne M.W. Janssen-Heininger 


\section{ABSTRACT}

S-glutathionylation has emerged as an oxidant-induced post-translational modification of protein cysteines which affects structure and function [1, 2]. The oxidoreductase, glutaredoxin-1 (Grx1) under physiological conditions catalyzes deglutathionylation and restores the protein thiol group. Our laboratory has previously demonstrated that Grx1 expression is increased in mice with ovalbumin induced-allergic airways inflammation [3], and that absence of Grx1 enhances the resolution of ovalbumin-induced inflammation and AHR. However, the impact of Grx1/S-glutathionylation on allergic inflammation induced by asthma-relevant allergens remains unknown. In the present study, we examined the impact of genetic ablation of the glutaredoxin-1 gene (GIrx1) on the pathogenesis of house dust mite (HDM)-induced allergic airways disease in mice. WT or Glrx $1^{\%}$ mice in the BALB/C

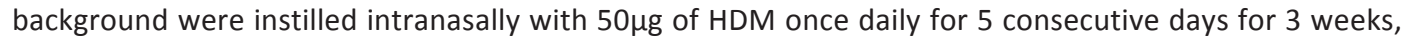
and were euthanized $72 \mathrm{~h}$ post final exposure. As expected, overall protein S-glutathionylation was increased in $\mathrm{Glrx}^{\%}$ mice exposed to HDM compared to WT animals. Total cells in the BAL fluid were similarly increased in WT and G/rx $1^{\%}$ HDM-treated mice compared to PBS controls. However, in response to HDM, mice lacking Glrx1 demonstrated significantly more neutrophils but less eosinophils as compared to respective WT animals. mRNA expression of IL-17A was significantly increased in the lungs of $\mathrm{G} / \mathrm{rx} \mathrm{H}^{\%} \mathrm{HDM}$ treated mice while expression of $\mathrm{T}_{\mathrm{H}} 2$-associated cytokines was attenuated. Similarly, cells isolated from the lungs of Glrx $1^{-}$HDM-exposed mice showed increased IL-17A production but decreases in IL-5 content in the media in vitro, as compared to cells from WT mice. Lastly, HDM-induced tissue stiffness and elastance were significantly attenuated in $\mathrm{Glr} \mathrm{1}^{\%}$ mice in addition to decreased mRNA expression of Muc5ac and IL-13 as well as IL-13 content within the lung, compared to WT littermates. These results demonstrate that the Grx1/S-glutathionylation redox status plays a pivotal role in HDM-induced allergic inflammation and airway hyperresponsiveness, and highlights the potential for Grx1 in controlling the nature of HDM-induced adaptive immune responses by promoting $T_{H} 2$ driven inflammation, and restricting IL-17A.

\section{Keywords}

Asthma, glutaredoxin-1, protein S-glutathionylation, IL-17A 


\section{INTRODUCTION}

Oxidative stress has been linked to the pathogenesis of a variety of diseases, and allergic inflammatory disorders such as chronic asthma are accompanied by changes in the oxidative environment [4]. Inflammatory cells recruited to the asthmatic airways have an exceptional capacity for producing a battery of reactive oxygen and nitrogen species. These molecules exert their biological activity through redox-based mechanisms, inflicting oxidative damage to biomolecules and modulating the activity of redox-sensitive signal transduction pathways [5]. The first line of defense against oxidants is an antioxidant defense system consisting of enzymatic and non-enzymatic antioxidants that include catalase, glutathione peroxidase (GPx), superoxide dismutase (SOD), peroxiredoxins, thioredoxins, vitamins $E$ and $\mathrm{C}$, uric acid and glutathione (GSH), among others. These antioxidant systems form a tightly regulated network that maintains the intra- and extracellular redox environment. Evidence for an oxidant-antioxidant imbalance in asthmatic airways is demonstrated in a number of studies, revealing decreased total antioxidant capacity as well as lower levels of individual antioxidants in plasma and bronchoalveolar lavage (BAL) fluid of patients with asthma [6]. Thiols in the form of sulfhydryl groups of proteins are among the most susceptible oxidant-sensitive targets and can be reversibly oxidized to sulfenic acids $(-\mathrm{SOH})$ or disulfides $(\mathrm{S}-\mathrm{S})$, or irreversibly oxidized to sulfinic $\left(-\mathrm{SO}_{2} \mathrm{H}\right)$ and sulfonic $\left(-\mathrm{SO}_{3} \mathrm{H}\right)$ acids.

S-glutathionylation (PSSG) represents an oxidant-induced post-translational modification of reactive cysteines within proteins, and has emerged as a key regulatory mechanism whereby oxidants regulate (patho-) biological processes. S-glutathionylation occurs when the sulfhydryl group present in glutathione (GSH) forms a mixed disulfide bond with a protein cysteine via mechanisms that remain incompletely understood. Increases in PSSG have been reported in different cell types under a variety of oxidative conditions [7], and numerous proteins have now been reported to be affected by $S$ glutathionylation. Glutaredoxin-1 (mammalian, cytosolic: Grx1) is a well-characterized, efficient and specific catalyst of deglutathionylation responsible in part for the reversal of PSSG under physiological conditions [7]. A member of the thiol-disulfide oxidoreductase family, Grx1 promotes reversible reduction of PSSG to free sulfhydryl groups through a monothiol mechanism, and is involved in a number of diseases including allergic airways disease. Our laboratories recently reported increases in Grx1 expression in both the sputum from asthmatic patients and the bronchiolar epithelium, in association with decreased PSSG [8]. These data correspond with the increases in Grx1 expression and 
activity observed predominantly in bronchial epithelium in the ovalbumin (OVA) model of allergic airways disease [1]. Mice lacking the glutaredoxin 1 gene (G/rx1) showed increases in PSSG and attenuation of airway inflammation and AHR in response to OVA compared to WT littermates, demonstrating that the Grx1/PSSG axis controls various manifestations of allergic airways [2].

The OVA model has facilitated the discovery of pathways and mediators relevant to the pathogenesis of allergic airways disease and human asthma, but controversy exists regarding the physiological relevance of this model. Ovalbumin is not believed to be an asthma-relevant allergen. The route of administration of ovalbumin and its adjuvant, alum, is via intraperitoneal injection. Alternative models have therefore been developed which have utilized more relevant allergens and sensitization methods. House dust mite (HDM) is a multifaceted allergen to which up to $85 \%$ of asthmatics are allergic [9]. To date, it is not clear whether the Grx1/PSSG redox axis controls the pathogenesis of HDM-induced allergic airways disease. The goal of the present study was to determine the impact of Glrx1 ablation on the development of HDM-induced allergic airways disease. Our results indicate that Grx1 may regulate HDM-induced adaptive immune responses by promoting $\mathrm{T}_{\mathrm{H}} 2$ driven inflammation and restricting IL-17A in allergic airways disease.

\section{MATERIALS AND METHODS}

Animal studies. Glrx $1^{-}$mice were backcrossed for $>10$ generations into a BALB/c background [10] (Jackson Laboratories) and were housed in the University of Vermont animal facility. For all experiments, 8-12 week old G/rx $1^{-1}$ and littermate WT BALB/c control mice were used. All procedures were approved by the Institutional Animal Care and Use Committee.

Murine model of HDM-induced allergic asthma. WT and Glr $\times 1^{-1}$ mice were subjected to intranasal instillations with $50 \mu \mathrm{g}$ of HDM extract (1.75 endotoxin units/50 $\mathrm{gg}$ of HDM extract normalized to protein content) resuspended in PBS, or with PBS alone as a vehicle control, once a day for 5 consecutive days for three weeks. Mice were euthanized $72 \mathrm{~h}$ following the final HDM challenge (Fig. 1A).

Assessment of AHR. All mice were anesthetized with an intraperitoneal injection of pentobarbital sodium $(90 \mathrm{mg} / \mathrm{kg})$, tracheotomized and mechanically ventilated at 200 breaths per minute. Respiratory mechanics were assessed at baseline (saline) and in response to increasing doses of methacholine (12.5, 
$25,50 \mathrm{mg}$ ) via a forced oscillation technique using a computer-controlled small animal ventilator (flexiVent $^{\circledR}$, SCIREQ) as previously described $[11,12]$.

Serum IgG1 and IgE. Following euthanization, blood was collected by heart puncture and immediately spun through a microtainer. Serum was collected and IgG1 and IgE content was determined using an ELISA-based method, with a 96-well plate pre-incubated with $1 \mu \mathrm{g} / \mathrm{ml}$ of HDM.

Bronchoalveolar lavage. Following euthanization, bronchoalveolar lavage (BAL) was collected using $1 \mathrm{ml}$ of PBS. Total cell counts were determined using the Advia ${ }^{\circledR} 120$ automated hematology analyzer. For differential cell counts, cells collected by BAL were centrifuged onto glass slides at $600 \mathrm{rpm}$, and cytospins were stained using the Hema3 kit (Fisher Scientific). A minimum of 300 cells were counted for every individual mouse, as previously described [12].

mRNA analysis. Lobes of the right lung were flash frozen, pulverized and total RNA was isolated and purified using the RNeasy kit (QIAGEN). 1 g of RNA was reverse transcribed to cDNA for TaqMan gene analysis using SYBR Green (Bio-Rad) to assess expression of chemokine (C-C motif) ligand 20 (CCL-20), Interleukin-13 (IL-13) and Interleukin-17A (IL-17A), Regulated on Activation, Normal T cell Expressed and Secreted (RANTES), and Mucin 5ac (MUC5ac). Expression values were normalized to the house keeping gene cyclophilin. Primers were as follows: CCL-20 (FW 5'-AAGACAGATGGCCGATGAAG-3', RV 5'ACGCCTTTTCACCCAGTTCT-3'), IL-13 (FW 5'-CCAGGCCCCTTCTAATGA-3', RV 5'GCCTCTCCCCAGCAAAGTCT-3'), IL-17A (FW 5'-TTTAACTCCCTTGGCGCAAAA-3', RV 5'CTTTCCCTCCGCATTGACAC-3'), RANTES (FW 5'-ATATGGCTCGGACACCACTC-3', RV 5'TCCTTCGAGTGACAAACACG-3'), MUC5ac (FW 5'-CAGTGAATTCTGGAGGCCAACAAGGTAGAG-3', RV 5'AGCTAAGCTTAGATCTGGTTGGGACAGCAGC-3') and cyclophilin (FW 5'-TTCCTCCTTCACAGAATTATTCCA-3', RV 5'-CCAGTGCCATTATGG-3').

Preparation of lung tissue for single-cell suspension. Lung cells were prepared using a lung dissociation kit (Miltenyi Biotec) and GentleMACs dissociator (Miltenyi Biotec) per manufacturer's instructions. Red

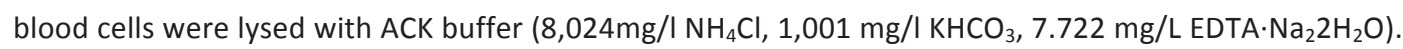
Suspensions were then spun and filtered through a $100 \mu \mathrm{m}$ (BD Biosciences) mesh filter. Cells were plated in $\mathrm{CD}^{+}$complete medium (5\% FBS (Cell Generation), pen/strep, L-glutamine, folic acid, glucose, 
and 2-ME in RPMI-1640 (Gibco) and either left untreated or restimulated with 15 $\mu \mathrm{g} / \mathrm{ml}$ of HDM for $96 \mathrm{~h}$ to elicit an immune response.

Preparation of splenoctyes. Splenocytes were harvested from WT and Glrx $1^{\%}$ mice. Single cell suspensions were generated by passing the tissues through a $70 \mu \mathrm{m}$ nylon mesh filter (BD Biosciences) and lymphocytes were enriched by centrifugation through Lymphocyte Separation Medium (MP Biomedicals). Cells were plated in $\mathrm{CD}^{+}$complete medium (5\% FBS (Cell Generation), pen/strep, Lglutamine, folic acid, glucose, and 2-ME in RPMI-1640 (Gibco) and either left untreated or restimulated with $15 \mu \mathrm{g} / \mathrm{ml}$ of HDM for $96 \mathrm{~h}$ to elicit an immune response.

Enzyme-linked immunosorbent assay. IL-5, IL-13, and IL-17A were detected by ELISA in lung homogenates (normalized for protein) according to the manufacturer's instructions (R\&D Systems).

Antibodies. The following antibodies were used in this study: mouse anti-GSH (Virogen), goat anti-Grx1 (American Immunoresearch) mouse anti $\beta$-actin (Sigma-Aldrich), secondary HRP-conjugated anti-mouse (Amersham), and secondary anti-goat (Jackson Immunoresearch).

Histopathology and myeloperoxidase immunohistochemistry. Following euthanization, left lung lobes were fixed with $4 \%$ paraformaldehyde in $\mathrm{ddH}_{2} \mathrm{O}$, paraffin-embedded and sectioned $(5-\mu \mathrm{m})$, and stained with Hematoxylin and Eosin (H\&E). Fixed sections were prepared for immunostaining by deparaffinizing with xylene and rehydrating through a series of ethanols. For antigen retrieval, slides were heated for $20 \mathrm{~min}$ in $95^{\circ} \mathrm{C}$ citrate buffer ( $\mathrm{pH} \mathrm{6.0)}$ with $0.05 \%$ Tween (Sigma-Aldrich) then rinsed with $\mathrm{ddH}_{2} \mathrm{O}$. Sections were then blocked in blocking serum (Vectastain) for $1 \mathrm{~h}$, followed by incubation with a primary antibody for myeloperoxidase (Abcam) overnight at $4^{\circ} \mathrm{C}$. Slides were washed with Tris $\mathrm{HCl}$ buffered saline with $0.1 \%$ Tween (TBSt) $3 \times 5 \mathrm{~min}$ each. Tissues were incubated with a universal, biotinylated, secondary antibody (Vectastain) for $30 \mathrm{~min}$ at room temperature and washed again $3 \times 5 \mathrm{~min}$ each. Vectastain ABCAP (Vectastain) reagents A and B were applied for $1 \mathrm{~h}$, and again washed. Reagents 1,2 , and 3 of the Vector Red Alkaline Phosphatase Substrate Kit 1 (Vectastain) were combined and added to the sections for approximately $20 \mathrm{~min}$. Slides were then rinsed with $\mathrm{dd}_{2} \mathrm{O}$, and counterstained with Hematoxylin. Slides were dehydrated and cleared through a number of xylene and ethanol baths, and coverslipped and mounted with PermaMount (Alban Scientific Inc.). 
Statistical analysis. All data were evaluated using Graphpad Prism 6 Software (Graphpad, Inc). Statistical significance was calculated with either a one-way ANOVA or two-way ANOVA $(P<0.05)$ and either a Bonferroni or Tukey post-hoc test.

\section{RESULTS}

Absence of glutaredoxin-1 leads to increases in protein S-glutathionylation (PSSG) in response to repeated HDM challenge. Based on our previous observations using the ovalbumin model of allergic airways disease, we first sought to determine whether Grx1 and PSSG were affected in a HDM model of allergic airways disease, using the intranasal sensitization regimen shown in Fig. 1A. In response to repeated intranasal administration of HDM, Grx1 expression was robustly increased in lung tissue homogenates of WT mice, compared to PBS vehicle controls (Fig. 1B). Under physiological conditions Grx1 acts to reduce target S-glutathionylated proteins to their sulfhydryl forms. Despite increases in Grx1, PSSG content was also increased in lung tissues following HDM exposure compared to PBS control mice. As expected, knockout of GIrx1 resulted in greater increases in overall PSSG in response to HDM compared to WT littermates (Fig. 1C).

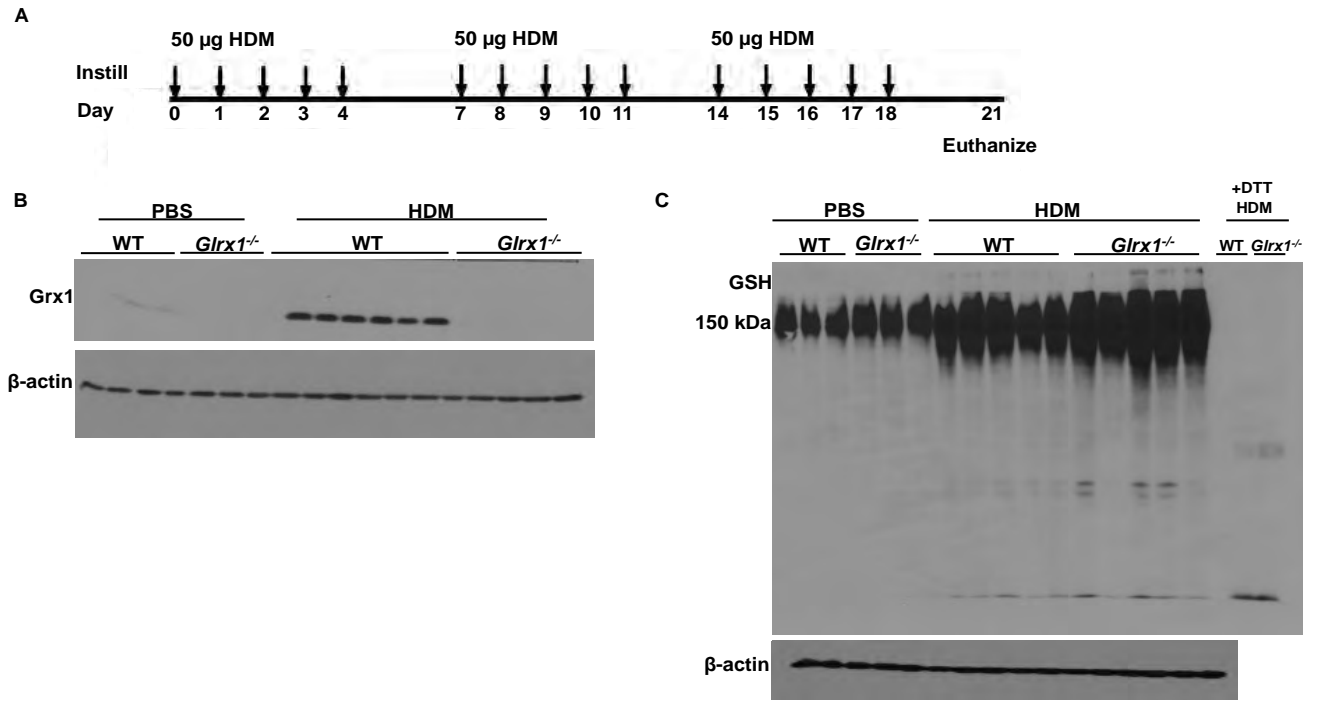

Figure 1. Evaluation of protein S-glutathionylation and Grx1 expression in the whole lung following HDM challenge. (A) Schematic depicting repeated intranasal instillation of $50 \mu \mathrm{g}$ of HDM or PBS for the vehicle control and euthanization on day $21,72 \mathrm{~h}$ following the final challenge. (B) Immunoblot for Grx 1 in homogenized lung tissue. $\beta$-actin is shown as a loading control. Immunoblots are representative of three independent experiments; WT PBS $n=3, G / r \times 1^{-}$PBS $n=4$, WT HDM $n=6, G / r \times 1^{-}$HDM $n=5$. (C) 
Immunoblot for total GSH in homogenized lung tissue. Lung tissue homogenates were electophoresed under non-reducing conditions, transferred into nitrocellulose and probed with an anti-GSH antibody. + DTT: representative samples from WT or G/rx $1^{\%}$ mice exposed to HDM incubated with DTT prior to electrophoresis, to reduce PSSG as a reagent control. $\beta$-actin is shown as a loading control. Immunoblots are representative of three independent experiments; WT PBS $n=3$, GIrx $1^{-1}$ PBS $n=3$, WT HDM $n=5$, Glrx $1^{-/}$HDM $n=5$.

Glrx $1^{-1}$ mice exhibit significantly increased neutrophilia accompanied by significant decreases in eosinophilia following HDM challenge, compared to littermate controls. The extent of HDM-induced inflammation was assessed by differential counts on cells collected by BAL (Fig. 2A) and by analyzing histopathology (Fig. 2C). Overall BAL cell counts were elevated to a similar extent in both WT and Glrx $1^{\%}$ mice compared to PBS controls (Fig. 2A, 2C). However, in HDM-challenged Glrx $1^{\%}$ mice, significantly more neutrophils were present in the BAL fluid compared to WT littermates (Fig. 2B). Increased myeloperoxidase (MPO) positive cells were also observed in lung tissues of G/rx $1^{\%}$ mice compared to WT animals (Fig. 2D, right panel). In contrast to the increases in airway and tissue neutrophils, however, airway eosinophilia in the lungs of $G / r \times 1^{-}$mice was considerably decreased compared to WT mice exposed to HDM. Total macrophages were also elevated in HDM challenged G/r $\times 1^{\%}$ mice compared to WT groups, while lymphocytes were comparable between both groups (Fig. 2B). Additionally, HDMinduced serum IgG1 was elevated to a similar extent in WT and Glrx $1^{-}$Balb/C mice compared to respective PBS controls. In contrast, serum IgE was significantly attenuated in Glrx $1^{\%}$ mice compared to HDM-exposed WT mice (Fig. 3). Overall these findings suggest that the nature of the inflammatory response to HDM is influenced by the Grx1/protein S-glutathionylation redox axis. 
A

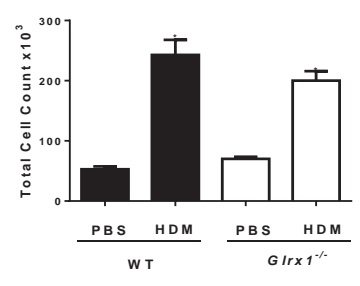

C

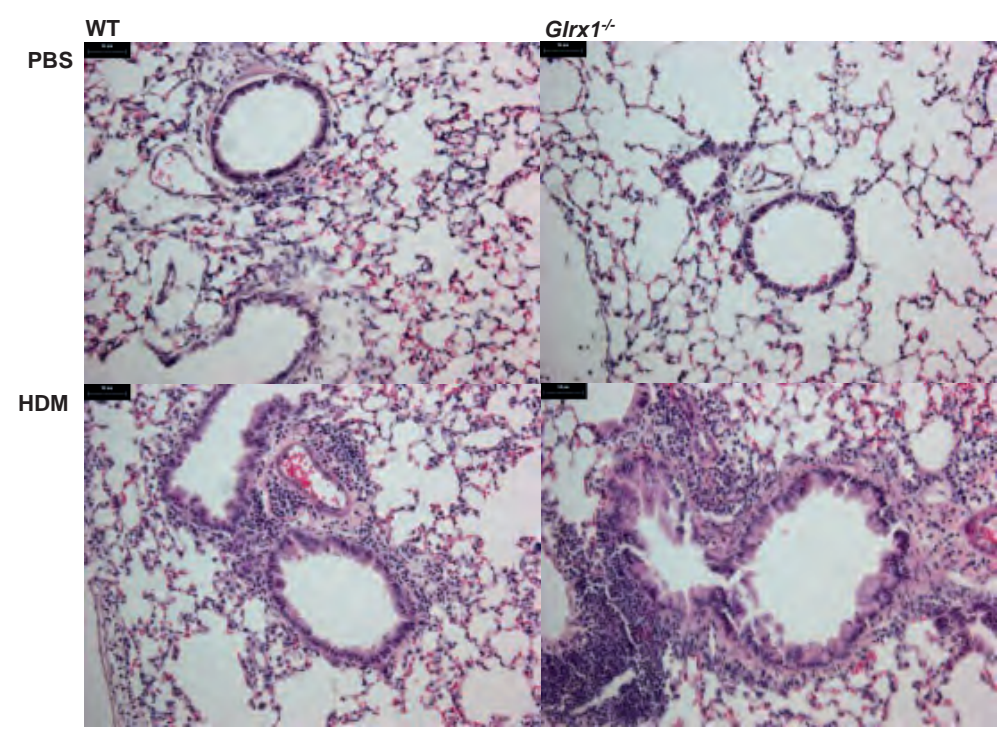

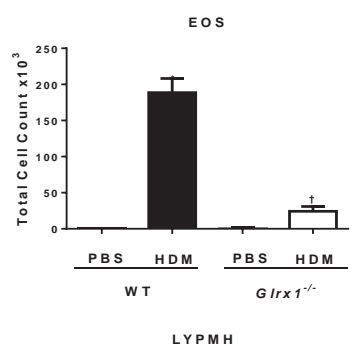
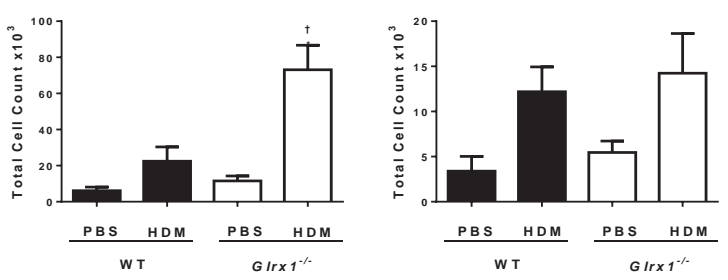
D
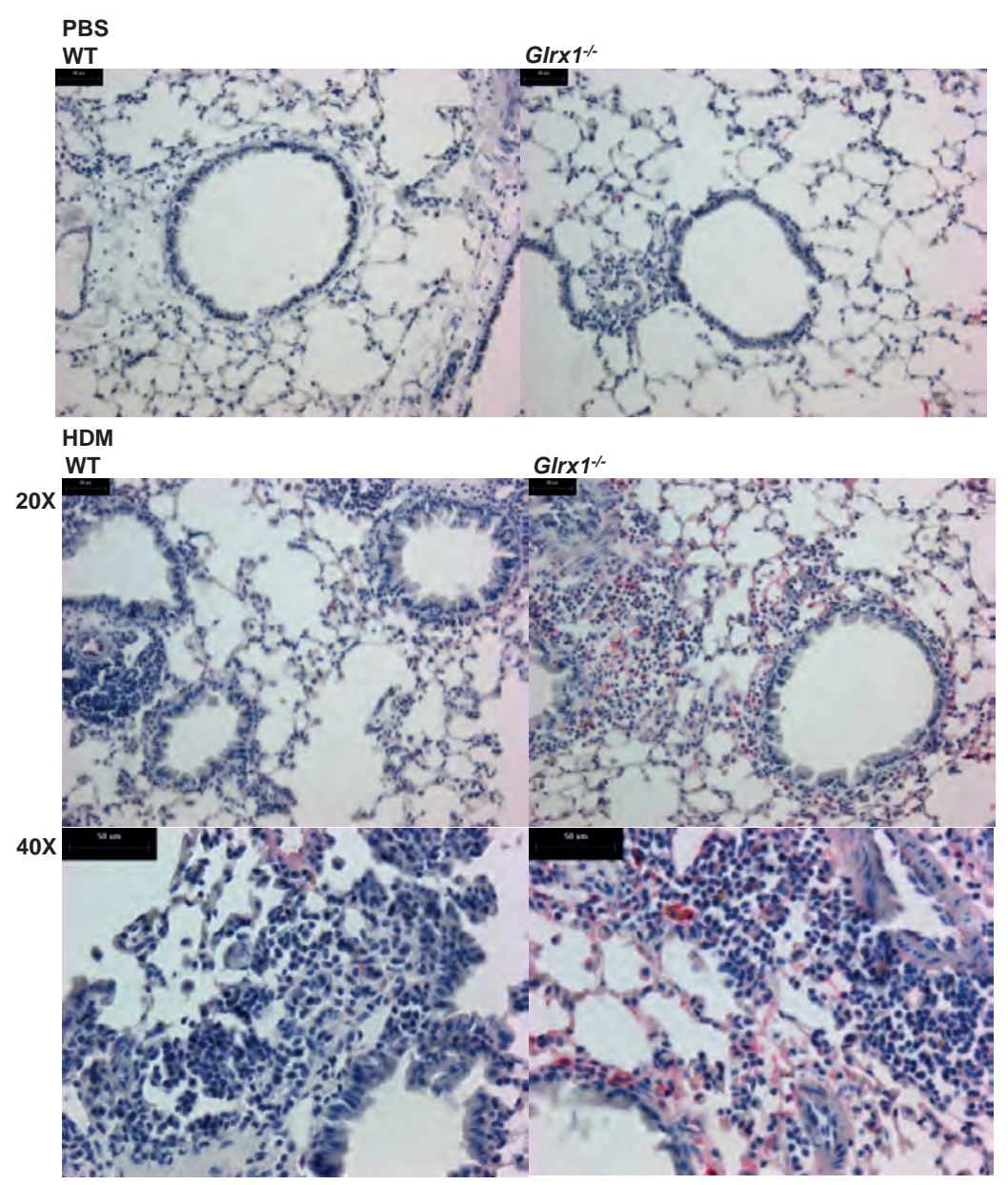

Figure 2. Evaluation of overall inflammation, and inflammatory cell differentials following 15 challenges of HDM. (A) Total and (B) differential cell counts in BAL from WT and G/rx $1^{-1-}$ mice following exposure to PBS or HDM. Total cell numbers were determined by Advia, and data are expressed as means \pm SEM (8-9 mice per group). ${ }^{*} p<0.05$ (ANOVA) compared with PBS controls; ${ }^{+} p<0.05$ (ANOVA) compared to WT mice challenged with HDM. (C) Histopathological evaluation of tissue inflammation (H\&E) from PBS or HDM-exposed mice. (D) Immunohistochemistry for myeloperoxidase (red). Images (20X top, 40X bottom) are representative of three independent experiments. Scale bars represent 50 $\mu \mathrm{m}$. 
A

$\lg E$

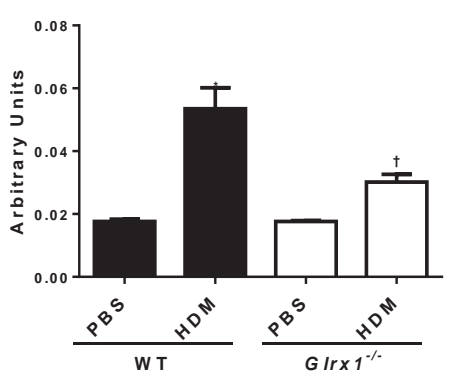

$\lg G 1$

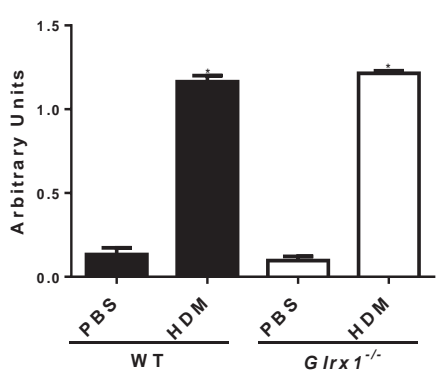

Figure 3. Assessment of HDM-induced serum IgG1 and IgE in WT and Glrx1/- mice. (A) Assessment of total HDM-specific IgE and IgG1 in the serum in WT and Glrx $1^{-1}$ mice. Serum immunoglobulin levels were measured by ELISA. Data are expressed as means \pm SEM of 6 mice per group. ${ }^{*} p<0.05$ (ANOVA) compared with PBS controls; $\uparrow p<0.05$ (ANOVA) compared with WT mice challenged with HDM.

Ablation of Glrx1 attenuates $T_{H} 2$ pro-inflammatory mediator expression and enhances IL-17A expression in the lung following HDM challenge. To further investigate the extent of HDM-induced allergic airway inflammation in WT and Glrx $1^{-1}$ mice, we evaluated mRNA expression of pro-inflammatory mediators in whole lung homogenates. mRNA expression of CCL-20, RANTES and IL-13 was increased in the lungs of WT mice following repeated HDM challenge however, these increases were not apparent in G/rx $1^{\%}$ mice (Fig. 4A). Furthermore, $\mathrm{T}_{\mathrm{H}} 2$-associated cytokines IL-13 and IL-5 were increased in the lungs of WT mice in response to HDM challenge however, production of these cytokines was significantly attenuated in Glrx $1^{-1}$ mice (Fig. 4B). Non- $\mathrm{T}_{\mathrm{H}} 2$ responses, such as IL-17A, E, or $\mathrm{F}$ production as well as neutrophil recruitment have been reported in the lungs of patients with asthma, particularly those with severe asthma or corticosteroid-resistant asthma [13]. In association with increased neutrophils recovered from the BAL fluid of GIrx $1^{-/}$mice, mRNA expression of IL-17A was also significantly elevated in response to HDM exposure, compared to WT mice (Fig. 4A). IL-17A content in the lung was undetectable in both WT and $G / r \times 1^{\%}$ mice $72 \mathrm{~h}$ following euthanization. These observations suggest that $\mathrm{T}_{\mathrm{H}} 17$-like responses may be enhanced while $\mathrm{T}_{\mathrm{H}} 2$ responses are dampened in $\mathrm{Glr} \times 1^{\%}$ mice exposed to HDM, as compared to WT littermate controls. 
A
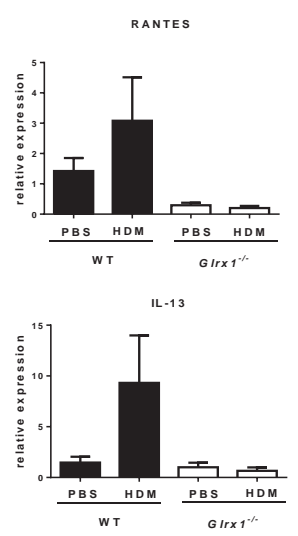
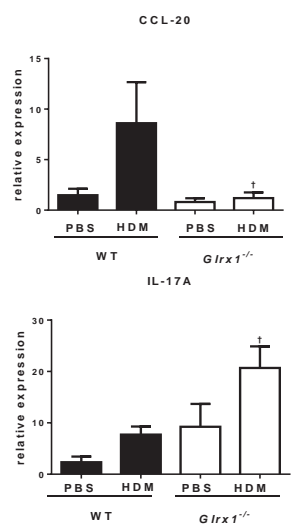
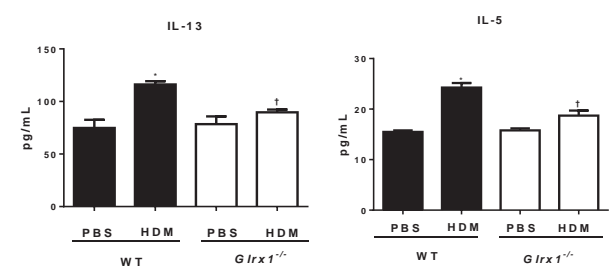

Figure 4. Analysis of HDM-induced pro-inflammatory mediator expression. (A) mRNA expression of RANTES, CCL-20, IL-13 and IL-17A from whole lung homogenates of WT and Glrx $1^{-1}$ mice was analyzed by real-time PCR. Results were normalized to the housekeeping gene cyclophilin. Data are expressed as fold increases in expression compared with WT PBS controls. Data are expressed as means \pm SEM of 6 mice per group. ${ }^{*} p<0.05$ (ANOVA) compared with PBS controls; ${ }^{\dagger} p<0.05$ (ANOVA) compared with WT mice challenged with HDM. (B) IL-13 and IL-5 content was measured in whole lung homogenates by ELISA. Data are expressed as means \pm SEM of 6 mice per group. ${ }^{*} p<0.05$ (ANOVA) compared with PBS controls; $\uparrow p<0.05$ (ANOVA) compared with WT mice challenged with HDM.

In order to further address these apparent differences, we prepared single cell suspensions from lung tissues, and addressed the spontaneous production of IL-5 or IL-17A (Fig. 5A), or following restimulation of cells with HDM (Fig. 5B) in vitro. Cells prepared from WT HDM-challenged mice spontaneously produced increased amounts of IL-5, compared to the PBS group. Compared to WT cells, spontaneous IL-5 production was significantly decreased in cells from Glrx $1^{\%}$ HDM-challenged mice (Fig. $5 \mathrm{~A})$. In response to re-stimulation of cells with HDM in vitro, IL-5 production further increased the cells from both WT and G/rx $1^{\%}$ HDM-challenged mice, although the overall amounts of IL-5 remained decreased in Glrx $1^{-/}$cells compared to respective WT cells (Fig. 5B). Spontaneous production of IL-17A was significantly increased in the media of cells isolated from Glrx $1^{\%} \mathrm{HDM}$-challenged mice, compared to WT cells. IL-17A produced by G/rx $1^{-1}$ cells was further increased following re-stimulation with $15 \mu \mathrm{g} / \mathrm{ml}$ of HDM in vitro compared to cells isolated from WT mice. In splenocytes isolated from HDMchallenged WT or Glrx $1^{-1}$ mice, upon re-stimulation with HDM in vitro, comparable increases in IL-5 were observed. In response to re-stimulation with HDM in vitro, small increases in IL-17A were apparent only in cells isolated from Glrx $1^{\%}$ mice. Overall, observed increases in response to HDM were blunted in splenocytes compared to those observed in lung cell suspensions. Collectively, these results 
demonstrate that the absence of GIrx1 results in enhanced IL-17A and dampened IL-5 cytokine production which occurred predominantly in cells of the lung.

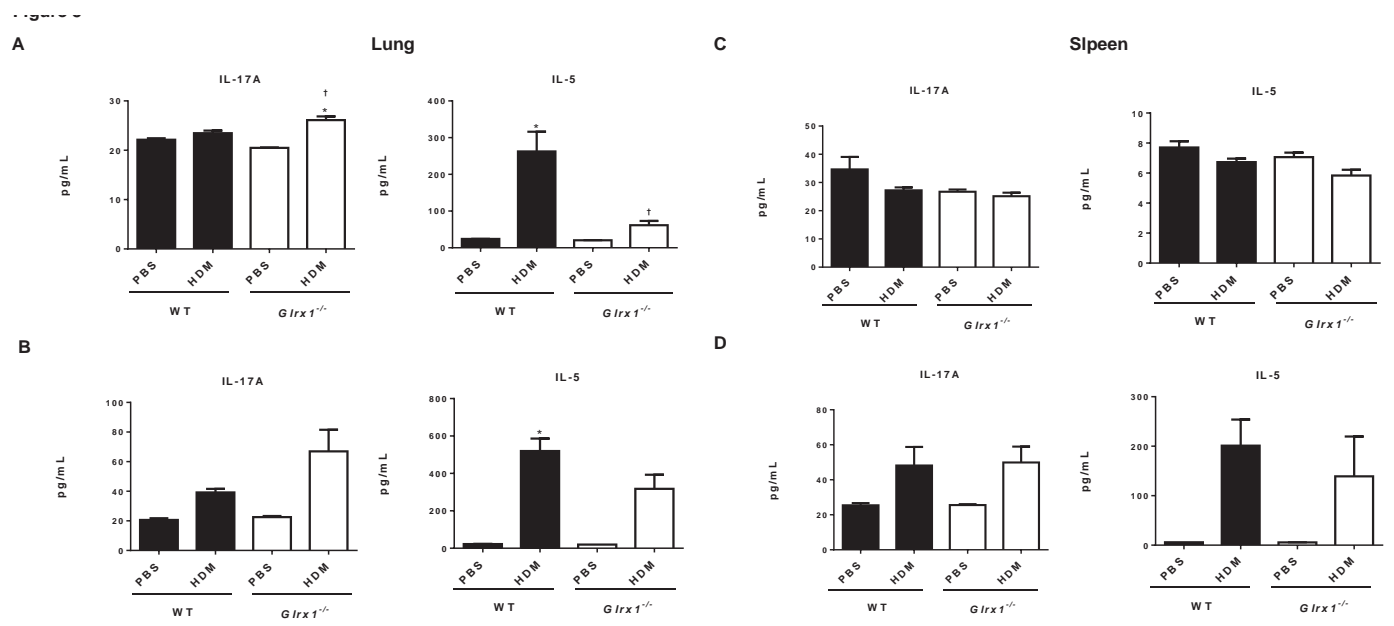

Figure 5. Assessment of HDM-induced cytokine expression and content in lung tissue from WT or Glrx $1^{\%}$ mice exposed to PBS or HDM. Media was collected from single lung cell $(A, B)$ or spleen suspensions $(C, D)$ from unstimulated (top) and cells re-stimulated (bottom) for $96 \mathrm{~h}$ with $15 \mu \mathrm{g} / \mathrm{ml}$ of HDM. IL-17A and IL-5 production was quantified by ELISA. Data are expressed as means \pm SEM (6 mice per group). ${ }^{*} p<0.05$ (ANOVA) compared with PBS controls; ${ }^{\prime} p<0.05$ (ANOVA) compared with WT mice challenged with HDM.

Attenuation of HDM-induced AHR and mucus metaplasia in Glrx $1^{-1}$ mice. To address the effects of ablation of Glrx1 on HDM-induced alterations in respiratory mechanics, a forced oscillation technique was used to evaluate responsiveness to ascending doses of methacholine. As expected, airway resistance $\left(R_{N}\right)$, tissue resistance $(G)$ and tissue elastance $(H)$ were significantly elevated in WT mice

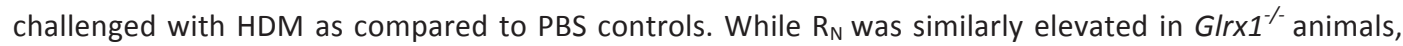
parameters $\mathrm{G}$ and $\mathrm{H}$, representing the peripheral airways were significantly decreased toward control values (Fig. 5A). Characteristic features of allergic airways disease include excess mucus production and goblet cell hyperplasia [11, 14], which have been shown to contribute to airway closure and hyperresponsiveness $[15,16]$. mRNA analysis of the mucin gene, MUC5ac revealed decreased expression in the lungs of Glrx ${ }^{-1}$ mice (Fig. 5B). However, histopathological analysis of lungs from WT and Glrx $1^{\%}$ mice revealed comparable HDM-induced mucus metaplasia (Fig. 5B). 
A

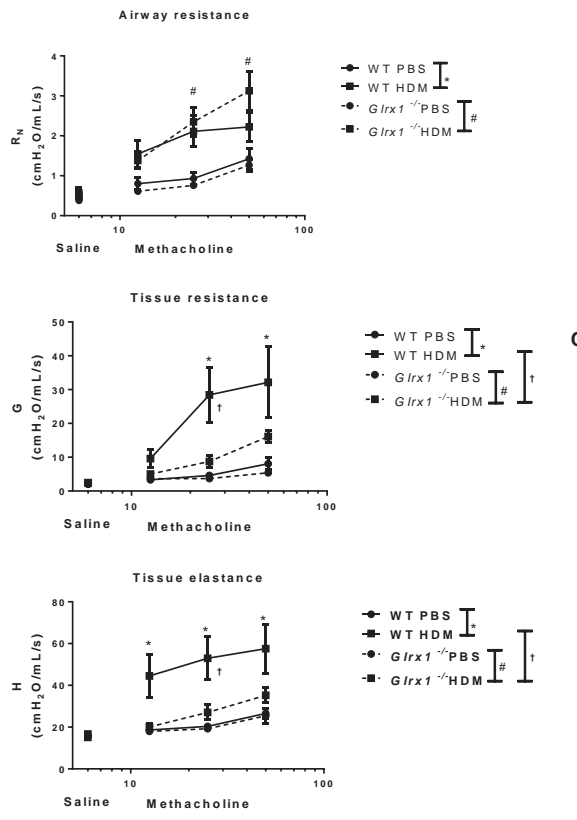

B
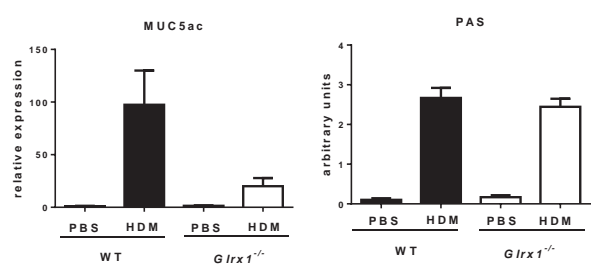

C

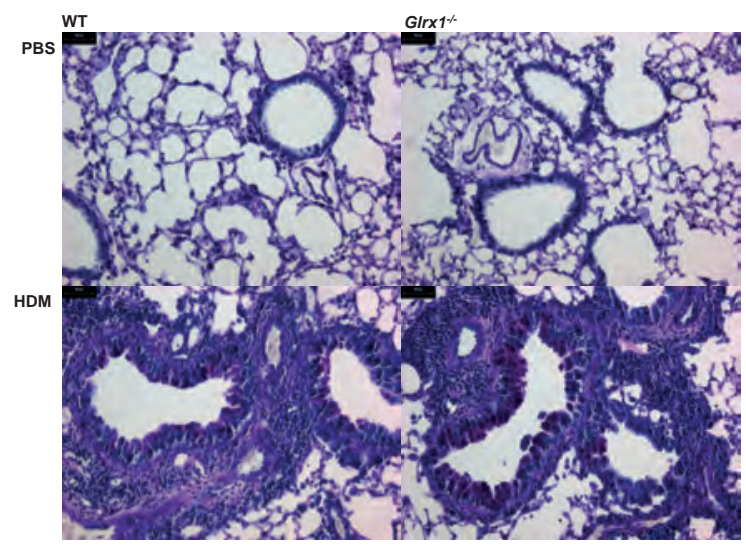

Figure 6. Evaluation of alterations in respiratory mechanics and mucin production in WT and GIrx $1^{\text {/- }}$ mice in response to 15 challenges of $50 \mu \mathrm{g}$ of HDM. (A) Assessment of airways hyperresponsiveness (AHR) via a forced oscillation technique in WT and $\mathrm{Glr}_{\mathrm{N}} \mathrm{1}^{-}$mice. Changes in respiratory mechanics were analyzed $72 \mathrm{~h}$ following the final instillation of either PBS or HDM. Ascending doses of methacholine were administered to determine Newtonian resistance $\left(R_{N}\right)$, tissue resistance $(G)$, and tissue elastance (H). Data are expressed as \% change from baseline measurements \pm SEM for each of the parameters measured from 6-8 mice per group. For AHR data, a three-way ANOVA was used with an additional term for methacholine dose. ${ }^{*} / \#<0.05$ compared with PBS controls; $+p<0.05$ compared with WT mice challenged with HDM. (B) Evaluation of MUC5ac gene expression in homogenized lung tissue. mRNA was analyzed by real-time PCR and results were normalized to the housekeeping gene cyclophilin and are expressed as fold increases in expression compared with WT PBS controls. Data are expressed as means \pm SEM (6 mice per group). ${ }^{*} p<0.05$ (ANOVA) compared with PBS controls; ${ }^{\dagger} p<0.05$ (ANOVA) compared with WT mice challenged with HDM. Mucus metaplasia was evaluated by obtaining 2 images of small bronchioles (X20 objective) of similar dimensions from each mouse. Average scores of 2 independent evaluations were determined. Data are expressed as means \pm SEM from 6-8 mice per group. ${ }^{*} p<0.05$ (Kruskal-Wallis) compared with respective PBS control groups. ${ }^{\dagger} p<0.05$ (Kruskal-Wallis) compared with WT HDM groups.

\section{Discussion}

The maintenance of redox homeostasis is crucial for appropriate functioning of cellular processes and cell survival. It is widely accepted that imbalances in the production and removal of reactive oxygen and nitrogen species (ROS-RNS) are associated with chronic inflammatory disorders such as asthma. 
Glutathione $(\mathrm{GSH})$ is the predominant endogenous antioxidant in mammalian cells and contributes to the redox homeostasis of the cell [17] and emerging studies suggest that the protein S-glutathionylation (PSSG) represents a mechanism whereby oxidants exert their (patho)physiological effects. Notably, the existence of glutaredoxins, which catalyze (de)glutathionylation reactions have given credence to the (patho)physiological role of PSSG [18]. Glutaredoxins have been implicated in a number of diseases, including allergic airways disease. Airways (sputum) of asthmatic patients displayed increased Grx1 and PSSG compared to non-asthmatics. Interestingly, a significant negative correlation between Grx1 and lung function was observed, suggesting an association between decreased Grx1, increased PSSG and improved lung function [8]. Our laboratory has previously demonstrated that the systemic loss of Glrx1 resulted in enhanced resolution of AHR and mucus metaplasia in antigen-induced allergic airways disease [2]. Results from the present study demonstrate that glutaredoxin-1 is increased in response to repeated HDM exposure (Fig. 1B) and acts to regulate HDM-induced airways inflammation and hyperresponsiveness. Moreover, we demonstrated clear increases in PSSG reactivity in lung tissues from Glrx $1^{-1}$ mice following $72 \mathrm{~h}$ HDM challenge (Fig $1 \mathrm{C}$ ), consistent with its role in catalysis of deglutathionylation reactions in physiological settings.

Asthma is a chronic, heterogeneous inflammatory disorder of the airways [19], and studies of patients and animal models suggest that $\mathrm{T}_{\mathrm{H}} 2$ cells that reside in the lung contribute to the persistence and progression of asthma $[20,21]$. The mouse model most commonly used to investigate allergic asthma involves sensitization by intraperitoneal injections of OVA complexed with the $T_{H} 2$ adjuvant, aluminum hydroxide (alum) [22]. This model has been valuable in studying $T_{H} 2$-mediated responses in allergic airway inflammation, but some aspects of the alum/OVA model do not reflect human asthma. The level of airway eosinophilia greatly exceeds that seen in human patients [22, 23], and neutrophil recruitment to the airways is transient [24] and shown to be dispensable for AHR [25]. Moreover, in humans, immune responses to inhaled allergens develop in the lung-draining lymph nodes, and the alum/OVA protocol bypasses this route via sensitization through the peritoneum. A more recent study demonstrated that sensitization through the airways induced a profoundly different immune response, and administration of a low dose of LPS as an adjuvant selectively primed modest $\mathrm{T}_{\mathrm{H}} 2$ responses and strong $T_{H} 17$ responses, prolonged neutrophilia and AHR [26]. In asthmatic patients, IL-17 expression is increased in the lungs, sputum, BAL fluid and sera, and correlates with the severity of AHR [27, 28]. However, mixed eosinophilic, neutrophilic and granulocytic mobilization and associated elevated total cell counts are often observed in the sputum of patients with severe allergic asthma [29]. 
In the present study, the extent of overall airway inflammation was increased comparably in both WT and Glrx $1^{-1}$ mice following repeated intranasal HDM administration (Fig. 2A, 2C), but clear differences in the type of infiltrating cells were apparent between the two strains (Fig. 2B). Infiltrating cells in the lungs of WT mice were predominantly eosinophils and lymphocytes, and to a lesser extent neutrophils. Conversely, neutrophils were significantly elevated in Glrx $1^{-/}$mice compared to WT mice (Fig. 2B, 2D). Interestingly, a recent study demonstrated that dynamic actin S-glutathionylation in neutrophils is regulated by Grx1, and overexpression of Grx1 impaired neutrophil migration [30]. It is not clear whether altered S-glutathionylation occurred in neutrophils and affected cellular function in the lungs of Glrx1-deficient animals, thereby contributing to HDM-induced allergic responses and altered respiratory mechanics. Further analysis is needed to determine the role of the Grx1/S-glutathionylation redox module in regulating actin dynamics and neutrophil recruitment in settings of allergic asthma.

In addition to significantly increased neutrophilia and decreased eosinophilia in Glrx $1^{-/}$mice, a potential shift away from the $\mathrm{T}_{\mathrm{H}} 2$-type responses was apparent in lungs from G/rx1-/- mice. Proinflammatory mediators such as IL-4, IL-5 and IL-13 produced by activated CD4 ${ }^{+}$T cells [31] stimulate increased IgE production in B cells [32]. WT mice demonstrated significantly elevated serum IgE in response to HDM (Fig. 3A) in addition to increased IL-5 and IL-13 content within the lung (Fig. 4B). Conversely, HDM-specific serum IgE as well as IL-5 and IL-13 were significantly attenuated in G/rx $1^{\%}$ mice. Consistent with increased neutrophilia, IL-17A mRNA expression was significantly increased following HDM exposure in GIrx $1^{\%}$ mice compared to WT mice (Fig. 4A). Moreover, re-stimulation of single lung cell suspensions with HDM revealed a trend towards enhanced IL-17A and dampened IL-5 production from HDM challenged Glrx $1^{-1}$ mice, as compared to WT littermates (Fig. 5A), responses that appeared blunted in single cell suspensions generated from the spleens (Fig. 5B), indicating that $\mathrm{T}$ cell activation and/or polarization might have preferentially taken place in the lung. Indeed, recent studies have shown that naïve T cells migrate to the peripheral tissues including the lung [33]. Furthermore, $\mathrm{CD}^{+} \mathrm{T}$ cell proliferation and $\mathrm{T}_{H} 17$ differentiation was shown to occur primarily in the lung and not the draining lymph nodes following bleomycin-mediated lung injury [34], suggesting that similar events might be operative in the present study. However, evaluation of $T_{H} 2$ and $T_{H} 17$ lineage-specific transcription factors GATA3 and RORyt from whole lung homogenates did not reveal any considerable differences between WT and GIrx $1^{-1}$ HDM-challenged mice (data not shown). In vitro polarization of naïve T cells isolated from WT and GIrx $1^{-/}$mice also failed to reveal significant differences in the capacity to differentiate towards $T_{H} 1, T_{H} 2$ and $T_{H} 17$ lineages (data not shown). Further analysis of cytokines 
promoting $T_{H}$ 17- differentiation and stabilization such as TGF $\beta$, IL-6, IL-1 $\beta$ and IL-23 [35] may also reveal how Grx1 and the protein thiol redox environment influences the nature of the inflammatory response.

Our group has previously demonstrated enhanced resolution of AHR in GIrx $1^{\%}$ mice following the ovalbumin (OVA) protocol, which corresponded to significant decreases in mucus metaplasia compared with WT mice [2]. In response to HDM, similar modulation of AHR was observed in Glrx $1^{\%}$ mice (Fig. 6A), with increases in $R_{N}$ being apparent, while tissue stiffness (damping) and elastance were significantly decreased compared to WT littermates. Thus, despite the differences in the antigens used, the route of sensitization, and inflammatory cell and cytokine milieu, absence of Glrx1 appears to affect AHR in a similar manner in both models. Despite the prior demonstration that mucus metaplasia was decreased in Glrx $1^{-}$mice using the OVA model, the present study showed comparable mucus metaplasia in WT and G/rx $1^{\%}$ mice subjected to HDM, despite the observed decreased MUC5ac mRNA expression in $\mathrm{Glrx}^{\%}$ mice compared to WT mice. The apparent discrepancy between these findings is likely attributable to the timing of evaluation of lung tissues following the final challenge with antigen, which differed. Of interest in this regard is the recent demonstration that airway mucins are targets for oxidation, manifested by disulfide-based crosslinks that increases mucin elasticity in association with pathological mucus gel formation [36]. It is not known at this time whether mucins are targets for $S$ glutathionylation, and whether this attenuates crosslinking. Such scenario could explain attenuation of AHR in $\mathrm{G} / r \times 1^{-1}$ mice, despite the apparent similar extent of overall mucus metaplasia. Previous studies have suggested that inflammation-triggered airway smooth muscle (ASM) contraction alone is not sufficient for airway hyperreactivity to methacholine, and that non-contractile factors such as mucus are essential for AHR [37].

Current pathological and physiological evidence suggests a role for the peripheral airways and parenchyma in the production of $\mathrm{T}_{\mathrm{H}} 2$ cytokines and airflow obstruction in asthmatics [38]. Increased numbers of IL-4 and IL-5-expressing cells were found within the small airways of resected lung specimens from asthmatics, and the expression of IL-5 mRNA was increased predominantly in the small airways [39]. Glrx $1^{\%}$ mice exhibited significantly decreased IL-5 protein content within the lung as compared to HDM-exposed WT mice. These results suggest that Grx1 may be involved in small airway immune responses and may contribute to peripheral airway hyperresponsiveness.

The exact cells and/or tissue compartments wherein Grx1/PSSG function to regulate allergic inflammation and AHR will require additional studies that incorporate in situ analysis of PSSG and cellspecific ablation of Glrx1. Similarly, identification of the proteins targeted via S-glutathionylation in HDM-induced lung disease and patients with asthma will also require additional analyses that 
incorporate Grx1-selective cysteine derivatization and detection via Mass Spectrometry [40], which was beyond the scope of the present study. The identification of a number of proteins that are known targets for S-glutathionylation [7] coupled with the emerging biological role of protein $S$ glutathionylation in (patho)biological processes [18] offer the potential towards the development of targeted protein-thiol based-therapeutics in order to alter the profile of allergic inflammation. 


\section{REFERENCES}

1. Reynaert, N.L., et al., Dynamic redox control of NF-kappaB through glutaredoxin-regulated Sglutathionylation of inhibitory kappaB kinase beta. Proc Natl Acad Sci U S A, 2006. 103(35): p. 13086-91.

2. Hoffman, S.M., et al., Genetic ablation of glutaredoxin-1 causes enhanced resolution of airways hyperresponsiveness and mucus metaplasia in mice with allergic airways disease. Am J Physiol Lung Cell Mol Physiol, 2012. 303(6): p. L528-38.

3. Reynaert, N.L., E.F. Wouters, and Y.M. Janssen-Heininger, Modulation of glutaredoxin-1 expression in a mouse model of allergic airway disease. Am J Respir Cell Mol Biol, 2007. 36(2): p. 147-51.

4. Andreadis, A.A., et al., Oxidative and nitrosative events in asthma. Free Radic Biol Med, 2003. 35(3): p. 213-25.

5. Loukili, N., et al., Oxidants positively or negatively regulate nuclear factor kappaB in a contextdependent manner. J Biol Chem, 2010. 285(21): p. 15746-52.

6. Nadeem, A., A. Masood, and N. Siddiqui, Oxidant--antioxidant imbalance in asthma: scientific evidence, epidemiological data and possible therapeutic options. Ther Adv Respir Dis, 2008. 2(4): p. 215-35.

7. Shelton, M.D. and J.J. Mieyal, Regulation by reversible S-glutathionylation: molecular targets implicated in inflammatory diseases. Mol Cells, 2008. 25(3): p. 332-46.

8. Kuipers, I., et al., Increased glutaredoxin-1 and decreased protein S-glutathionylation in sputum of asthmatics. Eur Respir J, 2013. 41(2): p. 469-72.

9. Gregory, L.G. and C.M. Lloyd, Orchestrating house dust mite-associated allergy in the lung. Trends Immunol, 2011. 32(9): p. 402-11.

10. Ho, Y.S., et al., Targeted disruption of the glutaredoxin 1 gene does not sensitize adult mice to tissue injury induced by ischemia/reperfusion and hyperoxia. Free Radic Biol Med, 2007. 43(9): p. 1299-312.

11. Riesenfeld, E., et al., The temporal evolution of airways hyperresponsiveness and inflammtion. Allergy and Therapy, 2012: p. 1-5.

12. Pantano, C., et al., Nuclear factor-kappaB activation in airway epithelium induces inflammation and hyperresponsiveness. Am J Respir Crit Care Med, 2008. 177(9): p. 959-69.

13. Kim, H.Y., R.H. DeKruyff, and D.T. Umetsu, The many paths to asthma: phenotype shaped by innate and adaptive immunity. Nat Immunol, 2010. 11(7): p. 577-84.

14. Morcillo, E.J. and J. Cortijo, Mucus and MUC in asthma. Curr Opin Pulm Med, 2006. 12(1): p. 1-6.

15. Singer, M., et al., A MARCKS-related peptide blocks mucus hypersecretion in a mouse model of asthma. Nat Med, 2004. 10(2): p. 193-6.

16. Evans, C.M., et al., Mucus hypersecretion in asthma: causes and effects. Curr Opin Pulm Med, 2009. 15(1): p. 4-11.

17. Xiong, Y., et al., S-glutathionylation: from molecular mechanisms to health outcomes. Antioxid Redox Signal, 2011. 15(1): p. 233-70.

18. Hoffman, S., et al., Thiol redox chemistry: role of protein cysteine oxidation and altered redox homeostasis in allergic inflammation and asthma. J Cell Biochem, 2015. 116(6): p. 884-92.

19. Anderson, G.P., Endotyping asthma: new insights into key pathogenic mechanisms in a complex, heterogeneous disease. Lancet, 2008. 372(9643): p. 1107-19.

20. Robinson, D.S., et al., Predominant TH2-like bronchoalveolar T-lymphocyte population in atopic asthma. N Engl J Med, 1992. 326(5): p. 298-304. 
21. Epstein, M.M., Targeting memory Th2 cells for the treatment of allergic asthma. Pharmacol Ther, 2006. 109(1-2): p. 107-36.

22. Kung, T.T., et al., Characterization of a murine model of allergic pulmonary inflammation. Int Arch Allergy Immunol, 1994. 105(1): p. 83-90.

23. Lex, C., et al., Airway eosinophilia in children with severe asthma: predictive values of noninvasive tests. Am J Respir Crit Care Med, 2006. 174(12): p. 1286-91.

24. Tomkinson, A., et al., Temporal association between airway hyperresponsiveness and airway eosinophilia in ovalbumin-sensitized mice. Am J Respir Crit Care Med, 2001. 163(3 Pt 1): p. 72130.

25. Taube, C., et al., Inhibition of early airway neutrophilia does not affect development of airway hyperresponsiveness. Am J Respir Cell Mol Biol, 2004. 30(6): p. 837-43.

26. Wilson, R.H., et al., Allergic sensitization through the airway primes Th17-dependent neutrophilia and airway hyperresponsiveness. Am J Respir Crit Care Med, 2009. 180(8): p. 720-30.

27. Molet, S., et al., IL-17 is increased in asthmatic airways and induces human bronchial fibroblasts to produce cytokines. J Allergy Clin Immunol, 2001. 108(3): p. 430-8.

28. Chakir, J., et al., Airway remodeling-associated mediators in moderate to severe asthma: effect of steroids on TGF-beta, IL-11, IL-17, and type I and type III collagen expression. J Allergy Clin Immunol, 2003. 111(6): p. 1293-8.

29. Simpson, J.L., et al., Inflammatory subtypes in asthma: assessment and identification using induced sputum. Respirology, 2006. 11(1): p. 54-61.

30. Sakai, J., et al., Reactive oxygen species-induced actin glutathionylation controls actin dynamics in neutrophils. Immunity, 2012. 37(6): p. 1037-49.

31. Webb, D.C., et al., Comparative roles of IL-4, IL-13, and IL-4Ralpha in dendritic cell maturation and CD4+ Th2 cell function. J Immunol, 2007. 178(1): p. 219-27.

32. Bosnjak, B., et al., Treatment of allergic asthma: modulation of Th2 cells and their responses. Respir Res, 2011. 12: p. 114.

33. Cose, S., et al., Evidence that a significant number of naive $T$ cells enter non-lymphoid organs as part of a normal migratory pathway. Eur J Immunol, 2006. 36(6): p. 1423-33.

34. Oh, K., et al., Epithelial transglutaminase 2 is needed for $T$ cell interleukin-17 production and subsequent pulmonary inflammation and fibrosis in bleomycin-treated mice. J Exp Med, 2011. 208(8): p. 1707-19.

35. Stockinger, B. and M. Veldhoen, Differentiation and function of Th17 T cells. Curr Opin Immunol, 2007. 19(3): p. 281-6.

36. Yuan, S., et al., Oxidation increases mucin polymer cross-links to stiffen airway mucus gels. Sci Transl Med, 2015. 7(276): p. 276 ra27.

37. Evans, C.M., et al., The polymeric mucin Muc5ac is required for allergic airway hyperreactivity. Nat Commun, 2015. 6: p. 6281.

38. Tulic, M.K., P. Christodoulopoulos, and Q. Hamid, Small airway inflammation in asthma. Respir Res, 2001. 2(6): p. 333-9.

39. Minshall, E.M., J.C. Hogg, and Q.A. Hamid, Cytokine mRNA expression in asthma is not restricted to the large airways. J Allergy Clin Immunol, 1998. 101(3): p. 386-90.

40. Guo, J., et al., Resin-assisted enrichment of thiols as a general strategy for proteomic profiling of cysteine-based reversible modifications. Nat Protoc, 2014. 9(1): p. 64-75. 


\section{CHAPTER 6}

Summary and Future Directions 


\section{Endoplasmic Reticulum Stress in Allergic Airways Disease}

Allergic asthma is characterized by airways inflammation, mucus metaplasia and peri- bronchiolar fibrosis, impacting lung structure and function [1, 2]. Airway epithelial cells (AECs) reside at the intersection of the lung and the external environment [3], and recent studies have demonstrated that activation of a number of receptors on the surface of AECs and subsequent secretion of various mediators are required for responses from dendritic cells (DCs) and subsequent immune responses [1, 4-6]. House Dust Mite (HDM) contains numerous antigens, proteases and ligands for Pattern Recognition Receptors (PRRs) resulting in activation of airway epithelial cells, and inducing the secretion of growth factors and cytokines that regulate subsequent activation of innate lymphoid cells, T cells, mucus metaplasia, inflammation, airways hyperresponsiveness (AHR), and fibrosis [7-9]. As discussed in Chapter 1, during allergen stimuli a demand for increases in protein synthesis and folding (eg. cytokines or mucus production) can create an imbalance in the endoplasmic reticulum (ER). This leads to an increase in misfolded proteins in the ER, causing ER stress and initiating the UPR signaling [10].

Studies thus far from our laboratory and others have shown that ER stress-dependent activation of transcription factors XBP1 and ATF6 $\alpha$ are required during mucus metaplasia and pro-inflammatory responses respectively, in ovalbumin or HDM-induced allergic airways disease [11, 12]. As discussed in Chapter 2, both cleavage product of ATF6 $\alpha$ and ERp57 protein are up regulated in murine and human epithelial cells in association with caspase-3 activation, following HDM exposure. siRNA-mediated knockdown of ATF6 $\alpha$ and ERp57 during HDM administration in mice resulted in a decrease in components of HDM-induced ER stress, disulfide mediated oligomerization of Bak, and activation of caspase-3. However, while siRNA also led to decreased inflammation, airway hyperresponsiveness and airway fibrosis, the impact of UPR-mediated induction of ATF6 $\alpha$ or ERp57 specifically has not been characterized in the development of allergic asthma. Furthermore, it is not clear whether allergeninduced ATF6 $\alpha$ or ERp57 is directly linked to multiple facets of asthma such as inflammation, apoptosis, peri-bronchiolar fibrosis and impairment in respiratory mechanics.

Substantial evidence suggests that the persistence of asthma is driven by ongoing immune responses that generate mediators driving airway remodeling and airway dysfunction. As has been discussed, the epithelium is both a site of production of these mediators and a source of cells that respond to mediators produced by immune cells and other cells within the airway, and understanding how epithelial cells recognize and respond to allergens and other stimuli is critical. Our laboratory has recently embarked on the projects utilizing ATF6 $\alpha$ systemic knock out mice and a doxycyline (Dox) 
inducible triple transgenic CCSP-rTetA/TetO-Cre/ERp57loxp/loxp ( $\triangle E$ pi-ERp57) mouse to delete ERp57 specifically in lung epithelial cells, to determine whether deletion of ATF6 $\alpha$ or ERp57 in mice or specifically in airway epithelial cells respectively attenuates pathophysiology associated with HDM challenge. Being a protein disulfide isomerase with specificity towards glycoproteins [13] (eg. cytokines and growth factors), it can be hypothesized that ERp57 could be involved in formation of disulfide bonds (-S-S-) in epithelial derived cytokine and chemokine production, affecting innate and adaptive immune responses following HDM exposure. Unraveling the pro-inflammatory mediators that are targets of ERp57 represents a major goal of future investigation.

\section{Glutaredoxin-1 and OVA-induced Allergic Airways Disease}

Glutaradoxin-1 (Grx1), an oxidoreductase responsible for protein deglutathionylation, is primarily expressed in the lung epithelium and in alveolar macrophages [14, 15]. Alterations in Grx1 expression have been associated with inflammatory diseases of the lung such as COPD and asthma [16, 17], and studies from our laboratory found significantly elevated levels of Grx1 within the airways of mice following OVA immunization and challenge [18]. To further elucidate the function of Grx1 in allergic airway disease, we determined the effects of ablation of the glutaredoxin-1 gene $\left(G / r \times 1^{-1}\right)$ in the OVA model. In response to OVA sensitization and challenge, Glr $\times 1^{\%}$ mice displayed decreased eosinophilic inflammation, mucus metaplasia, as well as enhanced resolution of airway hyperresponsiveness to methacholine. Glrx $1^{-/}$mice also exhibited increased protein S-glutathionylation in the lung [19] following repeated antigen challenge. As discussed in Chapter 1, a study by Kuipers, et al., reported decreased lung function associated with higher sputum Grx1 levels and lower PSSG levels [17], indicating that the Grx1/PSSG redox module may regulate AHR in allergic airways disease. While increases in overall protein S-glutathionylation were seen in the lungs of $G / r \times 1^{\%}$ mice exposed to OVA, the targets of $S$-glutathionylation remain unidentified. Future studies utilizing antibodies that target $S$ glutathionylated proteins or strategies to selectively reduce and label S-glutathionylated proteins [20] coupled with mass spectrometry will be required to identify these targets.

While the OVA model is widely used to study allergic airways disease, controversy exists regarding its physiological relevance. Both ovalbumin and the adjuvant, alum, are administered intraperitoneally in this model, but asthmatic patients are believed to be sensitized via the airway. Our laboratory therefore sought to utilize an alternative and perhaps more clinically relevant model of 
allergic airways disease to further study protein S-glutathionylation and Grx1 in the context of allergic airways disease, as described in Chapter 3.

\section{Epithelial NF-KB in HDM-Induced Allergic Airways Disease}

House dust mite (HDM) is a multifaceted allergen to which $50-85 \%$ of asthmatics are allergic [7]. HDM extract contains many components including fecal matter, LPS, chitin, and proteins such as Derp1, all of which are hypothesized to be involved in the allergic response. Previous studies with HDM demonstrated strong hyperresponsiveness to methacholine, inflammation, mucus metaplasia, and fibrotic remodeling in the mouse lung [21]. Additionally, HDM-induced allergic airway disease in mice via TLR4 triggering of airway structural cells, as well as production of pro-inflammatory cytokines TSLP, GM-CSF, IL-25, and IL-33 [4]. Utilization of the HDM model has enabled the investigation of early innate immune responses in the lung which are thought to contribute to the development of allergic airway disease.

Nuclear Factor-KB (NF-KB) is a family of transcription factors involved in the regulation of prosurvival, pro-inflammatory, and immune pathways. Dysregulation of NF-KB has been linked to a variety of chronic inflammatory diseases including cancer, sepsis, and asthma. NF-KB activity is elevated in lung epithelial cells of asthmatic patients in comparison to healthy controls [22], and activation of classical NF-KB in the lung epithelium is both sufficient and necessary to regulate allergic airway inflammation in mice $[6,23,24]$. The data presented in Chapter 3 demonstrate that HDM administration via the airways induces activation of both classical and alternative NF-KB, and that NF-KB inhibition in the epithelium protects against HDM-induced inflammation, hyperresponsiveness, and remodeling. Additionally, inhibition of NF-KB decreased the expression of IL-33, a cytokine known to promote $\mathrm{T}_{\mathrm{H}} 2$ differentiation activation of both the NF-KB and MAP Kinase pathways. Increased IL-33 expression has been demonstrated in the lungs of patients refractory to classic anti-inflammatory treatments, highlighting the potential therapeutic relevance of epithelial driven allergic airways disease.

As previously mentioned in Chapter 2, both classical and alternative NF-KB are inhibited in the epithelium following expression of the NF-KB SR transgene. Given the knowledge that NF-KB Rel proteins bind to ankyrin repeat domains on IKB's, this was is not surprising. Further experiments were necessary to define the independent contributions of the classical and alternative pathways in HDM-driven allergic disease. Utilizing RelB siRNA, we demonstrated that attenuation of the alternative pathway conveyed partial protection against HDM-induced pro-inflammatory cytokine expression. siRNA administration is 
not specific to the epithelium, however, and future studies might involve specific genetic models to further address the role of alternative NF-KB in the lung epithelium. Crossing a NIK/IKK $\alpha / R_{\text {elB }}{ }^{\text {flox/flox }}$ mouse with the CC10-rtta tetOP/Cre mouse, for example, might help clarify how activation of alternative NF-KB within epithelial cells regulates HDM-driven allergic airways disease.

Oxidant-induced post-translational modifications strongly influence NF-KB signaling, and key enzymes such as Grx1 control the extent of cysteine oxidation. NF-KB proteins are targets for Sglutathionylation in part via Grx1 [25], so mice which either inducibly overexpress Grx1 in CC10 positive epithelial cells, or which harbor a floxed Grx1 allele, could be used to selectively decrease Grx1 expression in the airway epithelium. These experiments would address the epithelial cell-specific effects of Grx1 for NF-kB activation, NF-KB driven allergic inflammation, and subsequent downstream effects such as inflammation, mucus production and AHR. Studies using chimeric mice in which wild-type and GIrx1-deficient animals are irradiated and their bone marrow repopulated with marrow of the opposite genotype could also demonstrate whether Grx1 expressed in structural cells of the lung, including epithelial cells, plays a role in allergic disease, and further identify potential targets for PSSG in epithelial cells and redox-based regulation in the orchestration of inflammatory responses.

\section{Glutaredoxin-1 and $\mathrm{T}_{\mathrm{H}} 17$ Driven Allergic Asthma}

Given the beneficial effects of global Grx1 knockout on mucus metaplasia and resolution of airway hyperresponsiveness observed in Chapter 2, and the role of NF-KB in HDM-induced allergic airways disease discussed in chapter 3, our group next investigated the role of Grx1 and protein $S$ glutathionylation in the pathophysiology of HDM-driven allergic airways disease. WT and Glrx $1^{\%}$ mice

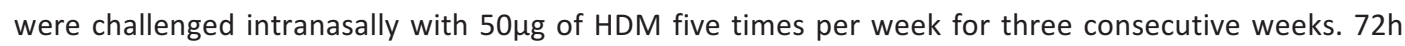
following the final challenge, WT mice exhibited prominent $T_{H} 2$-type inflammation, increased mucus metaplasia and AHR. Repeated HDM exposure increased Grx1 expression in the whole lung, and led to robust increases in PSSG in WT mice. Although we have not yet investigated the role of other deglutathionylating enzymes like Srx, it is clear that the level to which HDM induced PSSG could still not be overcome by endogenous antioxidants and reducing enzymes. As expected, in the absence of Grx1, overall PSSG was further increased as compared to WT mice. Analytical detection and quantification of glutathionylated proteins has been made easier by advances in proteomics and mass spectrometry. The results from Fig. 1C show prominent glutathionylation of proteins in the lung ranging from 100-150kDa, and mass spectrometry may be used to identify these proteins and provide further insight into the role of oxidative modifications in the pathogenesis of allergic airways disease. 
One of the most striking results from this study was the apparent shift away from the $T_{\mathrm{H}} 2$-type response seen in WT mice towards a $T_{H} 17$-like phenotype in the absence of Glrx1. Neutrophils were significantly elevated in G/rx $1^{-\%}$ mice in response to HDM challenge as compared to WT mice, while eosinophils were markedly decreased. Moreover, IL-17A mRNA expression in the whole lung as well as cytokine production from single lung cell suspensions was increased in the absence of Glrx1, while IL-5 production in the lung was significantly dampened. Severe asthma differs from mild or moderate persistant asthma, as it is characterized by neutrophilic inflammation either in the presence or absence of classical $T_{H} 2$-induced eosinophilic inflammation. Additionally, $T_{H} 17$ cells, which mediate neutrophil recruitment, are thought to have an influential role in asthma pathogenesis, especially in asthmatics who do not respond to glucocorticoid therapy [26]. Neutrophils are known to be largely steroidinsensitive and glucocorticoids (GC) inhibit neutrophil apoptosis [27] however, it is still unclear which cellular and molecular mechanisms contribute to this steroid insensitivity. Apart from $T_{H} 17$ cells, multiple cell types such as macrophages, $\gamma \delta T$ cells, as well as CD1d-restricted (i)NKT and innate lymphoid 3 cells (ILC3s) have previously been shown to be important sources of IL-17 in the lung [28-30], and future experiments will attempt to identify the cellular source of IL-17 in response to HDM in Glrx $1^{\%}$ mice. Furthermore, IL-17A has been shown to drive AHR in mice [31] and although hyperresponsiveness was dampened in the distal airways of $G / r \times 1^{-1-}$ mice, central airway resistance $\left(R_{N}\right)$ was increased to a magnitude similar to HDM-challenged WT mice. An IL-17A neutralizing antibody may therefore provide insight into the functional role of IL-17A in the absence of Grx1. If IL-17A does in fact play a significant role in HDM-induced allergic airways inflammation and AHR in $\mathrm{Glr} \times \mathrm{I}^{-}$mice, it would be interesting to address whether or not Grx1 ablation confers steroid insensivity, thus potentially illuminating a novel mechanism by which Grx1 and PSSG regulates disease type and severity. Interestingly, IL-17A is known to enhance chemotaxis of neutrophils by inducing IL-8 production in human bronchial epithelial cells [32] and airway smooth muscle cells [33]. Therefore, additional in vitro experiments might pinpoint a role for Grx1 in $T_{H} 17$-mediated glucocorticoid-insensitive inflammation.

Finally, as previously mentioned, G/rx $1^{-1}$ mice demonstrated signifiantly decreased tissue resistance $(\mathrm{G})$ and elastance $(\mathrm{H})$, as well as decreased Muc5ac mRNA expression compared to HDMtreated WT mice. These data, combined with results described in Chapter 2, highlight a potential functional role for Grx1 and PSSG in regulating mechanisms underlying airway hyperreactivity. Further immunohistological evaluation of the small and large airways may reveal regional variations in Grx1 expression and PSSG reactivity, and the predominant cell type(s) in which Grx1 is expressed. All 
together, these experiments may implicate a potential role for Grx1 and protein thiol oxidation, notably S-glutathionylation, in the pathogenesis of allergic asthma. 


\section{REFERENCES}

1. Lambrecht, B.N. and H. Hammad, The airway epithelium in asthma. Nat Med, 2012. 18(5): p. 684-92.

2. Anderson, G.P., Endotyping asthma: new insights into key pathogenic mechanisms in a complex, heterogeneous disease. Lancet, 2008. 372(9643): p. 1107-19.

3. Holtzman, M.J., et al., The role of airway epithelial cells and innate immune cells in chronic respiratory disease. Nat Rev Immunol, 2014. 14(10): p. 686-98.

4. Hammad, H., et al., House dust mite allergen induces asthma via Toll-like receptor 4 triggering of airway structural cells. Nat Med, 2009. 15(4): p. 410-6.

5. Rate, A., et al., Airway epithelial cells regulate the functional phenotype of locally differentiating dendritic cells: implications for the pathogenesis of infectious and allergic airway disease. J Immunol, 2009. 182(1): p. 72-83.

6. Ather, J.L., et al., Airway epithelial NF-kappaB activation promotes allergic sensitization to an innocuous inhaled antigen. Am J Respir Cell Mol Biol, 2011. 44(5): p. 631-8.

7. Gregory, L.G. and C.M. Lloyd, Orchestrating house dust mite-associated allergy in the lung. Trends Immunol, 2011. 32(9): p. 402-11.

8. Ryu, J.H., et al., Distinct TLR-mediated pathways regulate house dust mite-induced allergic disease in the upper and lower airways. J Allergy Clin Immunol, 2013. 131(2): p. 549-61.

9. Lan, R.S., G.A. Stewart, and P.J. Henry, Role of protease-activated receptors in airway function: a target for therapeutic intervention? Pharmacol Ther, 2002. 95(3): p. 239-57.

10. Tabas, I. and D. Ron, Integrating the mechanisms of apoptosis induced by endoplasmic reticulum stress. Nat Cell Biol, 2011. 13(3): p. 184-90.

11. Schroeder, B.W., et al., AGR2 is induced in asthma and promotes allergen-induced mucin overproduction. Am J Respir Cell Mol Biol, 2012. 47(2): p. 178-85.

12. Martino, M.B., et al., The ER stress transducer IRE1beta is required for airway epithelial mucin production. Mucosal Immunol, 2013. 6(3): p. 639-54.

13. Ellgaard, L. and E.M. Frickel, Calnexin, calreticulin, and ERp57: teammates in glycoprotein folding. Cell Biochem Biophys, 2003. 39(3): p. 223-47.

14. Peltoniemi, M., et al., Expression of glutaredoxin is highly cell specific in human lung and is decreased by transforming growth factor-beta in vitro and in interstitial lung diseases in vivo. Hum Pathol, 2004. 35(8): p. 1000-7.

15. Aesif, S.W., et al., In situ analysis of protein S-glutathionylation in lung tissue using glutaredoxin1-catalyzed cysteine derivatization. Am J Pathol, 2009. 175(1): p. 36-45.

16. Peltoniemi, M.J., et al., Modulation of glutaredoxin in the lung and sputum of cigarette smokers and chronic obstructive pulmonary disease. Respir Res, 2006. 7: p. 133.

17. Kuipers, I., et al., Increased glutaredoxin-1 and decreased protein S-glutathionylation in sputum of asthmatics. Eur Respir J, 2013. 41(2): p. 469-72.

18. Reynaert, N.L., E.F. Wouters, and Y.M. Janssen-Heininger, Modulation of glutaredoxin-1 expression in a mouse model of allergic airway disease. Am J Respir Cell Mol Biol, 2007. 36(2): p. 147-51.

19. Hoffman, S.M., et al., Genetic ablation of glutaredoxin-1 causes enhanced resolution of airways hyperresponsiveness and mucus metaplasia in mice with allergic airways disease. Am J Physiol Lung Cell Mol Physiol, 2012. 303(6): p. L528-38.

20. Reynaert, N.L., et al., In situ detection of S-glutathionylated proteins following glutaredoxin-1 catalyzed cysteine derivatization. Biochim Biophys Acta, 2006. 1760(3): p. 380-7. 
21. Johnson, J.R., et al., Continuous exposure to house dust mite elicits chronic airway inflammation and structural remodeling. Am J Respir Crit Care Med, 2004. 169(3): p. 378-85.

22. Hart, L.A., et al., Activation and localization of transcription factor, nuclear factor-kappaB, in asthma. Am J Respir Crit Care Med, 1998. 158(5 Pt 1): p. 1585-92.

23. Poynter, M.E., et al., NF-kappa B activation in airways modulates allergic inflammation but not hyperresponsiveness. J Immunol, 2004. 173(11): p. 7003-9.

24. Pantano, C., et al., Nuclear factor-kappaB activation in airway epithelium induces inflammation and hyperresponsiveness. Am J Respir Crit Care Med, 2008. 177(9): p. 959-69.

25. Reynaert, N.L., et al., Dynamic redox control of NF-kappaB through glutaredoxin-regulated Sglutathionylation of inhibitory kappaB kinase beta. Proc Natl Acad Sci U S A, 2006. 103(35): p. 13086-91.

26. McKinley, L., et al., TH17 cells mediate steroid-resistant airway inflammation and airway hyperresponsiveness in mice. J Immunol, 2008. 181(6): p. 4089-97.

27. Cox, G., Glucocorticoid treatment inhibits apoptosis in human neutrophils. Separation of survival and activation outcomes. J Immunol, 1995. 154(9): p. 4719-25.

28. Ley, K., E. Smith, and M.A. Stark, IL-17A-producing neutrophil-regulatory Tn lymphocytes. Immunol Res, 2006. 34(3): p. 229-42.

29. Michel, M.L., et al., Identification of an IL-17-producing NK1.1(neg) iNKT cell population involved in airway neutrophilia. J Exp Med, 2007. 204(5): p. 995-1001.

30. Song, C., et al., IL-17-producing alveolar macrophages mediate allergic lung inflammation related to asthma. J Immunol, 2008. 181(9): p. 6117-24.

31. Kudo, M., et al., IL-17A produced by alphabeta $T$ cells drives airway hyper-responsiveness in mice and enhances mouse and human airway smooth muscle contraction. Nat Med, 2012. 18(4): p. 547-54.

32. Jones, C.E. and K. Chan, Interleukin-17 stimulates the expression of interleukin-8, growth-related oncogene-alpha, and granulocyte-colony-stimulating factor by human airway epithelial cells. Am J Respir Cell Mol Biol, 2002. 26(6): p. 748-53.

33. Vanaudenaerde, B.M., et al., Interleukin-17 stimulates release of interleukin-8 by human airway smooth muscle cells in vitro: a potential role for interleukin-17 and airway smooth muscle cells in bronchiolitis obliterans syndrome. J Heart Lung Transplant, 2003. 22(11): p. 1280-3. 


\section{CHAPTER 7}

Valorisation, Dankwoord, Curriculum Vitae, \& Publication List 



\section{Valorisation}

This thesis is focused on the identification of redox mechanisms, notably the role of thiol oxidoreductases and protein thiol oxidation in human allergic asthma as well as murine models of allergic airways disease.

\section{Application: a role for glutaredoxin-1 and S-glutathionylation in epithelial cell apoptosis and fibrosis}

Additional studies from our laboratory have demonstrated the S-glutathionylation/glutaredoxin redox axis is an important module in the pathogenesis of multiple lung diseases. Pseudomonas aeruginosa, a well-studied ubiquitous pathogen commonly found in the lungs of cystic fibrosis and immunocompromised patients [1], also causes significant morbidity and mortality in critically ill ventilated patients [1]. Furthermore, activation of the death receptor, Fas, in the airway epithelium is a critical component of $P$.Aeruginosa clearance following infection [2, 3]. P.Aeruginosa produces numerous redox active compounds [4-6], and our group has demonstrated that S-glutathionylation of Fas enhances formation of the death-inducing signaling complex and accelerates apoptosis in epithelial cells and promoted clearance of Pseudomonas aeruginosa. Conversely, overexpression of Grx1 resulted in deglutathionylation of Fas, decreased FasL-induced apoptosis in lung epithelial cells, and decreased clearance of Pseudomonas aeruginosa [7], highlighting the importance of the precise balance of Fas-SSG and Grx1 [8].

Activation of Fas has also been shown to be causal in the pathogenesis of bleomycin (BLM)induced lung remodeling, in part by increasing epithelial cell apoptosis. Increased loss of epithelial cells is currently believed to play an important role in the pathogenesis of pulmonary fibrosis. Additional studies from our laboratory revealed increased protein S-glutathionylation within the bronchiolar epithelium [9], following oropharyngeal aspiration of bleomycin. Changes in glutathione content have also been previously reported in both rodent models of fibrosis and patients with IPF [10, 11] however, the extent to which protein S-glutathionylation, including Fas-SSG, is altered in these settings and contributes to disease pathogenesis, remains unknown. Investigation by our laboratory discovered a latent pool of Fas localized to the endoplasmic reticulum (ER) and upon activation of surface Fas, this separate pool was S-glutathionylated and shuttled to the surface, suggesting that FasL induces rapid changes in the redox status of the ER [12]. As previously mentioned, intramolecular disulfide (S-S) bond formation is catalyzed by protein disulfide isomerases (PDIs) [13-15], and stimulation with FasL resulted 
in a strong association between ERp57 and Fas. PDIs such as ERp57 also produce $\mathrm{H}_{2} \mathrm{O}_{2}[16,17]$, which is thought to promote protein S-glutathionylation via formation of a sulfenic acid intermediate. Glutathione S-transferases are classically known as phase II detoxifying enzymes that catalyze GSH conjugation reactions [18, 19], and recent reports suggest that GSTP1 can catalyze protein $S$ glutathionylation via the sulfenic acid intermediate during oxidative stress [20]. Our group further demonstrated that Fas-SSG was preceded by Fas-SOH, and Fas readily interacted with GSTP1 predominantly in the ER within 10 min of stimulation of with FasL [12].

To address the functional importance of ERp57, GSTP and Fas in oxidative processing, Sglutathionylation of Fas, and effects on epithelial cell apoptosis, ERp57 and GSTP1 were individually or simultaneously ablated using an siRNA approach or pharmacologically inhibited. While cells lacking ERp57 showed an almost complete loss of Fas-SSG, and a smaller yet consistent decrease following siRNA-mediated knockdown of GSTP1 upon Fas ligation, simultaneous ablation of both ERp57 and GSTP1 resulted in a complete loss of detectable Fas-SSG in response to FasL. Additionally, siRNA-based ablation of ERp57 and GSTP1 resulted in further decreased caspase- 3 and -8 activities and rescued cells from FasL-induced death compared to individual knockdown [12]. Incubation of epithelial cells with thiomuscimol, a known inhibitor of PDIs [21] significantly attenuated Fas-SSG in response to FasL compared to the inactive analog muscimol, with corresponding decreases in caspase- 3 and -8 activities. Incubation of cells with TLK199, a highly specific inhibitor of GSTP [22] also resulted in decreased FasLmediated Fas-SSG, caspase-3, and caspase-8 activities [12]. Taken together, these results demonstrate that the coordinated catalytic activities of ERp57 and GSTP contribute to Fas-SSG and activation of caspases.

Lung fibrosis is believed to be the result of dysregulated epithelial injury/repair mechanisms and aberrant myofibroblast activation and proliferation [23]. However, the mechanistic details whereby oxidative changes intersect with profibrotic signaling pathways remain elusive. Numerous pathways have been linked to the pathogenesis of fibrotic lung disease, including Fas [24, 25]. Immunoprecipitation of Fas from lung tissues of BLM-treated animals showed strong interactions with ERp57 and GSTP1, which were not detected in the PBS control group. In response to siRNA mediatedablation of ERp57 and GSTP1, mice showed significant decreases in collagen content in lung tissue 15 days following BLM exposure, as well as decreased Fas-SSG and caspase- 3 and -8 activities compared to Ctr siRNA-instilled mice [12]. Numerous signaling pathways leading to the progression of apoptosis have been identified. The studies from our laboratories discussed above serve to highlight how changes in the 
protein-thiol redox environment influence activation of cell death machinery, advancing our current understanding of cell death mechanisms.

As has been widely discussed in this dissertation, protein S-glutathionylation is increasingly recognized as an important mechanism underlying redox regulation of signaling pathways with downstream effects on numerous cell functions. It is known that S-glutathionylation of ER chaperones such as PDI regulates their function and induction of the unfolded protein response [26, 27], and recently ERp57 was shown to be glutathionylated in LPS-stimulated RAW264.7 mouse macrophages [28]. The evidence presented in this thesis suggests that ER stress, oxidative stress, and inflammatory responses are intimately linked and highlights the potential relevance of investigating the role of the Grx1 and S-glutathionylation of PDIs in epithelial cell ER stress, apoptosis and fibrotic remodeling in allergic asthma.

\section{Implementation: approach for future investigation}

The generation of reactive oxygen species is a complex process. The mechanisms of ROS generation and their subsequent impact are likely disease or context dependent. For years, accumulating evidence has clearly demonstrated that ROS are increased in various disease states. These findings imply that administration of antioxidants such as $\mathrm{N}$-acetylcysteine or glutathione would counteract these potentially harmful ROS and protect against disease. However, the therapeutic potential of antioxidants in clinical settings remains unsubstantiated [29]. Improved understanding the molecular pathways and targets responsible for oxidative damage may help clarify whether antioxidants are beneficial or harmful towards to treatment or prevention of diseases. A wealth of studies published over the last two decades has demonstrated that oxidants can function as signaling molecules, and are critical regulators of normal cellular processes including differentiation, growth, cell death and senescence [30,31]. Moreover, the release of oxidants from the mitochondria and other sources, can trigger a protective response that may protect the cell from additional stresses, a process known as hormesis or mitohormesis [32]. It is possible that interruption of this response may in part explain why antioxidants have had limited efficacy in clinical practice. Given this dichotomy regarding the potential effects of ROS, perhaps our investigations should shift away from distinguishing 'bad' from 'good' ROS, and refocus towards determining the extent to which aberrant production of ROS can be dampened, yet homeostatic functions are maintained. These investigations also should include oxidant-induced post-translational 
modifications of proteins, and be aimed at maintaining beneficial protein-S-glutathionylation targets, while reversing pathological S-glutathionylation reactions.

\section{Relevance: socioeconomic and environmental importance}

The prevalence of asthma varies significantly throughout the world, and the World Health Organization (WHO) estimates the number of asthma patients will increase by 100 million by 2025 [33]. Asthma is more prevalent among working age groups which can negatively impact productivity [34], and coupled with rising costs in patient care, asthma represents a major global economic burden [33]. Additionally, the emergence of new therapies and disease management strategies constitutes a significant cost component in industrialized countries, where medication is generally the largest driver of direct costs of asthma; in comparison, in-patient and out-patient care seem to be the major source of financial burden in developing regions [33]. Evidence suggests that the increasing cost of medication parallels a reduction in the costs and numbers of hospitalizations and patient-care visits [35-37], which may reflect better access to asthma control medications in more affluent countries. However, the landscape of healthcare systems across the world is dynamic, influenced not only by the emergence of new treatments but also evolving genetic, behavioral (e.g., change in smoking rates), and environmental risk factors which all contribute to the economic burden of this disease.

In addition to asthma prevalence, obesity has also dramatically increased in the past two decades and the convergence of these trends has prompted investigations into the relationship between obesity and asthma. A growing body of evidence suggests that obesity is an important risk factor for asthma [38] and prospective studies indicate a probable shared genetic basis for asthma and obesity [39]. Clinical investigations have demonstrated a very complex association between obesity and asthma [40], and suboptimal responses to conventional asthma therapy are often attributed to distinct immunological and physiological phenotypes in overweight patients compared to normal-weight individuals [41]. Some beneficial effects of weight loss on asthma control have been reported [42]; however, additional studies carefully phenotyping matched cohorts of obese and normal-weight subjects with and without asthma are needed to evaluate the impact of obesity on airway physiology and inflammation.

While the cellular and molecular mechanisms underlying the association of obesity and asthma have yet to be fully elucidated, attention is being brought to changes in the redox environment. Increased systemic or airway oxidative stress may be a potential cause for increased severity in the co- 
occurrence of obesity and asthma [43]. Supporting this notion, it has been reported that serum levels of GSH are markedly decreased in obese children [44]. Moreover, mice subjected to a high fat diet in the ovalbumin model of allergic airways disease exhibited significantly lower concentrations of GSH in the BALF and lung tissues, accompanied by increased transcriptional activity of NF-KB compared to nonobese asthmatic mice [45]. These reports provide further rationale to investigate the involvement of the S-glutathionylation/glutaredoxin redox axis in asthma and obesity, and a more thorough understanding of altered redox status will unveil novel mechanisms that can be exploited to develop more targeted therapies, ultimately resulting in better treatment options.

Lastly, it is widely accepted that the global environment of earth is radically shifting, and changes in air quality and climate have a quantifiable impact for both morbidity and mortality of asthma and other respiratory diseases [48]. A statement issued by the European Respiratory Society (ERS) (developed by members of Health and Environmental Network (HENVINET) and the American Thoracic Society) highlights climate change and related health impacts [49], specifically noting altered spatial and temporal distribution of allergens (pollens, molds and mites) [49]. While the effects of climate change on respiratory allergy are not fully known, increased exposure to allergens as a result of global climate change, combined with exposure to pollutants may act synergistically to enhance the allergic response resulting in more prominent and severe disease [50]. While this thesis approaches disease on a cellular and molecular level, the results presented herein add to the collective knowledge of the pathophysiological mechanisms driving allergic asthma. 


\section{REFERENCES}

1. Chastre, J. and J.Y. Fagon, Ventilator-associated pneumonia. Am J Respir Crit Care Med, 2002. 165(7): p. 867-903.

2. Grassme, H., et al., CD95/CD95 ligand interactions on epithelial cells in host defense to Pseudomonas aeruginosa. Science, 2000. 290(5491): p. 527-30.

3. Sadikot, R.T., et al., Targeted immunomodulation of the NF-kappaB pathway in airway epithelium impacts host defense against Pseudomonas aeruginosa. J Immunol, 2006. 176(8): p. 4923-30.

4. O'Malley, Y.Q., K.J. Reszka, and B.E. Britigan, Direct oxidation of 2',7'-dichlorodihydrofluorescein by pyocyanin and other redox-active compounds independent of reactive oxygen species production. Free Radic Biol Med, 2004. 36(1): p. 90-100.

5. O'Malley, Y.Q., et al., Pseudomonas aeruginosa pyocyanin directly oxidizes glutathione and decreases its levels in airway epithelial cells. Am J Physiol Lung Cell Mol Physiol, 2004. 287(1): p. L94-103.

6. Muller, M., Pyocyanin induces oxidative stress in human endothelial cells and modulates the glutathione redox cycle. Free Radic Biol Med, 2002. 33(11): p. 1527-33.

7. Anathy, V., et al., Redox amplification of apoptosis by caspase-dependent cleavage of glutaredoxin 1 and S-glutathionylation of Fas. J Cell Biol, 2009. 184(2): p. 241-52.

8. Anathy, V., et al., Glutaredoxin-1 attenuates S-glutathionylation of the death receptor fas and decreases resolution of Pseudomonas aeruginosa pneumonia. Am J Respir Crit Care Med, 2014. 189(4): p. 463-74.

9. Aesif, S.W., et al., In situ analysis of protein S-glutathionylation in lung tissue using glutaredoxin1-catalyzed cysteine derivatization. Am J Pathol, 2009. 175(1): p. 36-45.

10. Meyer, A., R. Buhl, and H. Magnussen, The effect of oral N-acetylcysteine on lung glutathione levels in idiopathic pulmonary fibrosis. Eur Respir J, 1994. 7(3): p. 431-6.

11. Rahman, I., et al., Systemic and pulmonary oxidative stress in idiopathic pulmonary fibrosis. Free Radic Biol Med, 1999. 27(1-2): p. 60-8.

12. Anathy, V., et al., Oxidative processing of latent Fas in the endoplasmic reticulum controls the strength of apoptosis. Mol Cell Biol, 2012. 32(17): p. 3464-78.

13. Appenzeller-Herzog, C. and L. Ellgaard, The human PDI family: versatility packed into a single fold. Biochim Biophys Acta, 2008. 1783(4): p. 535-48.

14. Jansens, A., E. van Duijn, and I. Braakman, Coordinated nonvectorial folding in a newly synthesized multidomain protein. Science, 2002. 298(5602): p. 2401-3.

15. Sevier, C.S. and C.A. Kaiser, Conservation and diversity of the cellular disulfide bond formation pathways. Antioxid Redox Signal, 2006. 8(5-6): p. 797-811.

16. Tu, B.P. and J.S. Weissman, The FAD- and O(2)-dependent reaction cycle of Ero1-mediated oxidative protein folding in the endoplasmic reticulum. Mol Cell, 2002. 10(5): p. 983-94.

17. Zhang, K. and R.J. Kaufman, From endoplasmic-reticulum stress to the inflammatory response. Nature, 2008. 454(7203): p. 455-62.

18. Boyland, E. and L.F. Chasseaud, The role of glutathione and glutathione S-transferases in mercapturic acid biosynthesis. Adv Enzymol Relat Areas Mol Biol, 1969. 32: p. 173-219.

19. Douglas, K.T., Mechanism of action of glutathione-dependent enzymes. Adv Enzymol Relat Areas Mol Biol, 1987. 59: p. 103-67.

20. Manevich, Y., S.I. Feinstein, and A.B. Fisher, Activation of the antioxidant enzyme 1-CYS peroxiredoxin requires glutathionylation mediated by heterodimerization with pi GST. Proc Natl Acad Sci U S A, 2004. 101(11): p. 3780-5. 
21. Hoffstrom, B.G., et al., Inhibitors of protein disulfide isomerase suppress apoptosis induced by misfolded proteins. Nat Chem Biol, 2010. 6(12): p. 900-6.

22. Lyttle, M.H., et al., Isozyme-specific glutathione-S-transferase inhibitors: design and synthesis. J Med Chem, 1994. 37(1): p. 189-94.

23. Janssen-Heininger, Y.M., et al., Regulation of apoptosis through cysteine oxidation: implications for fibrotic lung disease. Ann N Y Acad Sci, 2010. 1203: p. 23-8.

24. Aoshiba, K., et al., The Fas/Fas-ligand system is not required for bleomycin-induced pulmonary fibrosis in mice. Am J Respir Crit Care Med, 2000. 162(2 Pt 1): p. 695-700.

25. Wallach-Dayan, S.B., R. Golan-Gerstl, and R. Breuer, Evasion of myofibroblasts from immune surveillance: a mechanism for tissue fibrosis. Proc Natl Acad Sci U S A, 2007. 104(51): p. 20460-5.

26. Xiong, Y., et al., S-Glutathionylation of Protein Disulfide Isomerase Regulates Estrogen Receptor alpha Stability and Function. Int J Cell Biol, 2012. 2012: p. 273549.

27. Townsend, D.M., et al., Nitrosative stress-induced s-glutathionylation of protein disulfide isomerase leads to activation of the unfolded protein response. Cancer Res, 2009. 69(19): p. 7626-34.

28. Checconi, P., et al., Redox proteomics of the inflammatory secretome identifies a common set of redoxins and other glutathionylated proteins released in inflammation, influenza virus infection and oxidative stress. PLoS One, 2015. 10(5): p. e0127086.

29. Guallar, E., et al., Enough is enough: Stop wasting money on vitamin and mineral supplements. Ann Intern Med, 2013. 159(12): p. 850-1.

30. Schieber, M. and N.S. Chandel, ROS function in redox signaling and oxidative stress. Curr Biol, 2014. 24(10): p. R453-62.

31. Holmstrom, K.M. and T. Finkel, Cellular mechanisms and physiological consequences of redoxdependent signalling. Nat Rev Mol Cell Biol, 2014. 15(6): p. 411-21.

32. Yun, J. and T. Finkel, Mitohormesis. Cell Metab, 2014. 19(5): p. 757-66.

33. Ehteshami-Afshar, S., et al., The global economic burden of asthma and chronic obstructive pulmonary disease. Int J Tuberc Lung Dis, 2016. 20(1): p. 11-23.

34. FitzGerald, J.M. and M. Sadatsafavi, The importance of measuring asthma control in emerging economies. Int J Tuberc Lung Dis, 2014. 18(3): p. 254.

35. Jang, J., et al., Trends in cost and outcomes among adult and pediatric patients with asthma: 2000-2009. Ann Allergy Asthma Immunol, 2013. 111(6): p. 516-22.

36. Bedouch, P., et al., Trends in asthma-related direct medical costs from 2002 to 2007 in British Columbia, Canada: a population based-cohort study. PLoS One, 2012. 7(12): p. e50949.

37. Sadatsafavi, M., et al., Direct health care costs associated with asthma in British Columbia. Can Respir J, 2010. 17(2): p. 74-80.

38. Beuther, D.A. and E.R. Sutherland, Overweight, obesity, and incident asthma: a meta-analysis of prospective epidemiologic studies. Am J Respir Crit Care Med, 2007. 175(7): p. 661-6.

39. Beuther, D.A., S.T. Weiss, and E.R. Sutherland, Obesity and asthma. Am J Respir Crit Care Med, 2006. 174(2): p. 112-9.

40. Lugogo, N.L., M. Kraft, and A.E. Dixon, Does obesity produce a distinct asthma phenotype? J Appl Physiol (1985), 2010. 108(3): p. 729-34.

41. Scott, H.A., et al., Airway inflammation is augmented by obesity and fatty acids in asthma. Eur Respir J, 2011. 38(3): p. 594-602.

42. Juel, C.T., et al., Asthma and obesity: does weight loss improve asthma control? a systematic review. J Asthma Allergy, 2012. 5: p. 21-6.

43. Komakula, S., et al., Body mass index is associated with reduced exhaled nitric oxide and higher exhaled 8-isoprostanes in asthmatics. Respir Res, 2007. 8: p. 32. 
44. Albuali, W.H., Evaluation of oxidant-antioxidant status in overweight and morbidly obese Saudi children. World J Clin Pediatr, 2014. 3(1): p. 6-13.

45. Liu, X., et al., Correlation between oxidative stress and the NFkappaB signaling pathway in the pulmonary tissues of obese asthmatic mice. Mol Med Rep, 2015.

46. Calixto, M.C., et al., Obesity enhances eosinophilic inflammation in a murine model of allergic asthma. Br J Pharmacol, 2010. 159(3): p. 617-25.

47. Brashier, B. and S. Salvi, Obesity and asthma: physiological perspective. J Allergy (Cairo), 2013. 2013: p. 198068.

48. D'Amato, G., et al., Effects on asthma and respiratory allergy of Climate change and air pollution. Multidiscip Respir Med, 2015. 10: p. 39.

49. Stafoggia, M., et al., Factors affecting in-hospital heat-related mortality: a multi-city casecrossover analysis. J Epidemiol Community Health, 2008. 62(3): p. 209-15.

50. D'Amato, G., et al., Meteorological conditions, climate change, new emerging factors, and asthma and related allergic disorders. A statement of the World Allergy Organization. World Allergy Organ J, 2015. 8(1): p. 25. 


\section{Dankwoord}

At the close of this journey, I reflect upon the events that have led me here and show appreciation to those who have been un-ending in their support and guidance.

Firstly, I thank my promoter Prof. dr. Yvonne Janssen-Heininger for her mentorship, and for believing in my potential as a scientist nearly seven years ago. To Prof. dr. Emiel Wouters, it is because of your continued international collaboration that I will attain the degree of doctor from Maastricht University, a premier academic institution and for that I am deeply appreciative. Dr. Vikas Anathy, I can attribute both personal and professional achievements to yourself and your family. I have truly benefitted from your sense of calm and endless knowledge, and believe you will continue to influence a many number of successful scientists. To Prof. dr. Edward Dompeling, thank you for serving as the Chairman of the Evaluation Committee, and also to Prof. dr. Boris Kramer for your participation and continued professional kindness. I also thank Prof. dr. Guy Brusselle, Prof. dr. Louis Renaud, and Prof. dr. Aalt Bast for also acting on the Evaluation Committee. I look forward to regarding you all as colleagues, and hope to demonstrate my continued appreciation by making substantial contributions to the scientific and broader communities in the pursuit to alleviate disease.

I also acknowledge the significant role of family and friends in this grand pursuit. To my oldest brother Jason, who has acted as a redwood in my life. You are greatly selfless, and have always offered never ending strength and resourcefulness. Kerri, I aspire to have your compassion and strength, and hope to provide the guidance and support you have bestowed upon me, to your children. To my other half Brady, there is no one who has believed in my dreams longer than you, and you never forgot to get me roses. To my future sister in-law Amy, you have been a light in our lives and I am so very excited for what the future holds for our new family. The friends that I have made during this time are certainly my closest, and it is difficult to express here my gratitude for each of them. To Jane and Joanne, clearly the best relationships arise from ripping one's jeans, seeking out help from the nearest female colleague and getting some duck tape from her colleague's friend upstairs. Things certainly happen for a reason. My beloved Kendall, for someone who could be described as tightly wound, your loving free-spirit is everything I ever needed to enjoy the flavors of life and I hope to have a lifetime of adventures with you, even if it means eating paprika toast. To Bridget, Adam, Audrey, Brian, Justin, and Marge whom like me profess their love (and sometimes hatred) of Ullr, thank you so much for sharing in the joys of the mountains and taking me to new heights! Japan is next. 
Finally, I have my grandmother Louise G. Hoffman to thank for providing for my education. Her intelligence, beauty and strength is unmatched and I have been so fortunate to have her love and support. To my parents, there is nothing other than to say and so many things to say it for but, thank you and I love you. Jimmy and Blue, you both are my life and my love and I would not be at this place without you. To all of those who were not mentioned yet have been a part of my journey, I thank you. 


\section{Curriculum vitae}

Sidra Marie Hoffman was born on March $6^{\text {th }} 1987$ in Burlington, Vermont, USA. She attended Middlebury Union High School in Middlebury, Vermont from 2001-2005. During that time, Sidra Hoffman was a principal dancer with the Vermont Chamber Ballet where she performed feature and/ or principle roles in classics such as Don Quixote, La Bayadere, Swan Lake, The Nutcracker and The Sleeping Beauty. She went on to train at the Jose Mateo Ballet Theatre in Cambrige, Massachusetts as well as the Central Pennsylvania Youth Ballet, under the direction of Marcia Dale Weary. In 2004, she was awarded a gold medal for a solo performance at the National Dance Educators of America competition in New York, New York.

Sidra attended the University of Utah in Salt Lake City, Utah from 2005-2009 and studied exercise physiology within the College of Health. Following her undergraduate studies, she was accepted into the environmental pathology program within the department of Pathology and Laboratory Medicine at the University of Vermont. Under the mentorship of Dr. Yvonne Janssen-Heininger, she studied the role of glutaredoxin-1 in allergic airways disease, and completed her MSc in July of 2011. Sidra was then offered to continue her research as a doctoral candidate in a unique collaboration between Maastricht University and the University of Vermont under the co-mentorship of Dr. Emiel Wouters and Dr. Yvonne Janssen-Heininger. In 2013, Sidra joined the laboratory of Dr. Vikas Anathy where she investigated endoplasmic reticulum stress in the pathogenesis of allergic asthma and fibrotic airways remodeling. During her time at the University of Vermont, Ms. Hoffman was awarded a scholarship from the March of Dimes foundation to attend the $54^{\text {th }}$ annual short course on medical and experimental mammalian genetics at Jackson Laboratories in Bar Harbor, Maine. She also received an abstract award from the American Thoracic Society- Assembly on Allergy, Immunology and Inflammation, and in May of 2015 was invited to speak under the topic of "novel pathways in pulmonary fibrosis" at the annual meeting of the American Thoracic Society in Denver, Colorado. Sidra now lives in Seattle, Washington and is pursuing a career in science. 


\section{Publications List}

1. Hoffman S.M., Qian X., Chapman D.G., Lahue K.G., Schneider R., Ather J.L., Nolin J.D., McMillan D.H., Jones J.T., Taatjes D.J., Aliyeva M, Daphtary N, Abdalla S., Lundblad L.K.A., Ho YS, Anathy V, Irvin C.G., Wouters E.F.M., Reynaert N.L., Dixon A.E., van der Vliet A., Poynter M.E. and Janssen-Heininger Y.M.W. Ablation of glutaredoxin-1 modulates house dust mite-induced allergic airways disease in mice. Am J Resp Cell Mol Biol. Under revision.

2. Hoffman S.M., Chapman D.G., Lahue K.G., Cahoon J, Rattu G, Daphtary N, Aliyeva M, Fortner K, Erzurum, S.C., Comhair S.A.A., Woodruff P.G., Dixon A.E., Irvin C.G., Janssen-Heininger Y.M.W., Poynter M.E., and Anathy V. Protein Disulfide Isomerase-ERp57 regulates allergen-induced airways inflammation, fibrosis and hyperresponsiveness. J Allergy Clin Immunol. 15: 01242-7 PMCID: PMC4597791.

3. Hoffman S.M., Nolin J.D., McMillan D.H., Wouters E.F.M., Janssen-Heininger Y.M.W. and Reynaert N.L. Thiol redox chemistry: role of protein cysteine oxidation and altered redox homeostasis in chronic allergic inflammation. Invited perspective for J Cell Biochem. 116: 884-92 PMCID: PMC4620985.

4. Nolin J.D., Tully J.E., Hoffman S.M., Guala A.S., van der Velden J.L., Poynter M.E., van der Vliet A, Anathy V, Janssen-Heininger Y.M. (2014) The glutaredoxin/S-glutathionylation axis regulates interleukin-17A induced proinflammatory responses in lung epithelial cells in association with Sglutathionylation of nuclear factor KB family proteins. Free Radic Biol Med. 73: 143-53. PMCID: PMC4111997.

5. van der Velden J.L.J., Hoffman S, Alcorn J.F., Tully J, Chapman D, Lahue K, Guala A, Lundblad L.K.A., Irvin C.G., Janssen-Heininger Y.M.W. (2014) Absence of c-Jun N-terminal kinase 1 protects against house dust mite-induced pulmonary remodeling, but not airway hyperresponsiveness and inflammation. Am J Physiol Lung Cell Mol Physiol. 306: L866-75. PMCID: PMC4010650.

6. Hoffman S, Tully J, Nolin J, Lahue K, Guala A, Goldman D, Daphtary N, Aliyeva M, Irvin C.G., Dixon A, Poynter M.E., Janssen-Heininger Y.M.W., Anathy V. (2013) Endoplasmic reticulum stress mediates house dust mite-induced airway epithelial apoptosis and fibrosis. Respir Res. 24:141. PMCID: PMC3877992.

7. Anathy V*, Aesif S.W.*, Hoffman S*, Bement J.L., Guala A, Lahue K, Leclair L.W., Suratt B.T., Cool C.D., Wargo M.J., Janssen-Heininger Y.M.W. * Co-first author (2014) Glutaredoxin-1 attenuates Sglutathionylation of the death receptor fas and decreases resolution of pseudomonas aeruginosa pneumonia. Am J Respir Crit Care Med. 189: 463-74. PMCID: PMC3977722.

8. Tully J, Hoffman S, Lahue K, Nolin J, Anathy V, Lundblad L.K.A., Daphtary N, Aliyeva M, Black K, Dixon A, Poynter M.E., Irvin C.G., Janssen-Heininger Y.M.W. (2013) Epithelial NF-kappaB orchestrates house dust mite-induced inflammation, airway hyperresponsiveness and fibrotic airways remodeling in the lung. J Immunol. 191: 5811-21. PMCID: PMC3858534.

9. Janssen-Heininger Y.M.W., Nolin J, Hoffman S, Tully J, Lahue K, Abdalla S, Chapman D.G., Reynaert N.L., van der Vliet A, Anathy V. (2013) Emerging mechanisms of glutathione-dependent chemistry in biology and disease. J Cell Biochem. 114: 1962-8. PMCID: PMC3857728.

10. Tully J, Nolin J, Guala A, Hoffman S, Roberson E, Lahue K, van der Velden J.L.J., Anathy V, Blackwell T.S., Janssen-Heininger Y.M.W. (2012) Cooperation between classical and alternative NF-kappaB pathways regulates proinflammatory responses in epithelial cells. Am J Respir Cell Mol Biol. 47: 497-508. PMCID: PMC3488618.

11. Hoffman S, Tully J, Lahue K, Anathy V, Nolin J, Guala A, van der Velden J.L.J., Ho Y.S., Aliyeva M, Daphtary N, Lundblad L.K.A., Irvin C.G., Janssen-Heininger Y.M.W. (2012) Genetic ablation of 
glutaredoxin-1 causes enhanced resolution of airways hyperresponsiveness and mucus metaplasia in mice with allergic airways disease. Am J Physiol Lung Cell Mol Physiol. 303: L52838. PMCID: PMC3468477.

12. Anathy V, Roberson E, Cunniff B, Nolin J, Hoffman S, Spiess P, Guala A, Lahue K, Goldman D, Flemer S, van der Vliet A, Heintz N, Budd R.C., Tew K.D., Janssen-Heininger Y.M.W. (2012) Oxidative processing of latent fas in the endoplasmic reticulum controls the strength of apoptosis. Mol Cell Biol. 32: 3464-78. PMCID: PMC3422013.

\section{Abstracts/Presentations}

Hoffman S.M., Chapman D.G., Lahue K.G., Cahoon J, Daphtary N, Aliyeva M, JanssenHeininger Y.M.W., Poynter M.E., Anathy V. Epithelial-specific deletion of protein disulfide isomerase-ERP57 leads to significant attenuation of HDM-induced fibrotic airways remodeling and hyperresponsiveness. American Thoracic Society, May 2015.

Cunniff B, Cahoon J.M., Hoffman S.M., Taatjes D.J., Bouffard N.A., Poynter M.E., Heintz N.H., Anathy V. Endoplasmic reticulum (ER) and mitochondrial interactions during house dust mite induced inflammatory response. American Thoracic Society, May 2015.

Chapman D.G., van der Velden J.L.J., Lahue K.G., Hoffman S.M., Daphtary N, Aliyeva M, Janssen-Heininger Y.M.W., Irvin C.G. Nuclear factor KB persistent activation in airway epithelial cells in a mouse model of allergic airways disease induces persistence of neutrophilia and AHR. Thoracic Society of Australia and New Zealand, March 2015.

McMillan D.H., Lahue K.G., Nolin J.D., Hoffman S.M., Abdalla S, Chapman D.G., van der Velden J.L.J., Anathy V, Janssen-Heininger Y.M.W. Attenuation of GSTP1 decreases Fas Sglutathionylation-mediated lung epithelial cell apoptosis and fibrotic remodeling. Society for Free Radical Biology and Medicine, Seattle, WA. November 2014.

Hoffman S, Nolin J, Tully J, Lahue K, Aliyeva M, Daphtary N, Lundblad L.K.A., Chapman D, Ho Y.S., Irvin C.G., Anathy V, Janssen-Heininger Y.M.W. Genetic ablation of glutaredoxin-1 in a model of house dust mite-induced allergic asthma modulates airway neutrophilia, IL-17A and AHR. American Thoracic Society, May 2014.

Chapman D.G., van der Velden J.L.J., Hoffman S, Lahue K, Tully J, Tracy R.P., Worthen G.S., Poynter M.E., Janssen-Heininger Y.M.W., Irvin C.G. Increased expression of duffy antigen receptor for chemokines in a murine model of allergic airways disease is associated with persistence of airway hyperresponsiveness. American Thoracic Society, May 2014.

Hoffman S, Tully J, Nolin J, Lahue K, Guala A, Goldman D, Daphtary N, Aliyeva M, Irvin C.G., Dixon A, Poynter M.E., Janssen-Heininger Y.M.W., Anathy V. Endoplasmic reticulum stress mediates house dust mite-induced airway epithelial apoptosis and fibrosis. $54^{\text {th }}$ annual Short Course on Medical and Experimental Mammalian Genetics, Jackson Laboratories Bar Harbor ME, July 2013.

Tully J, Hoffman S, Lahue K, Nolin J, Lundblad L.K.A., Daphtary N, Aliyeva M, Poynter M.E., Irvin C.G., Janssen-Heininger Y.M.W. Activation of classical and alternative nuclear factor- 
kappaB in lung epithelium orchestrates chronic house dust mite-induced inflammation, airways hyperresponsiveness, and fibrotic airways remodeling. American Thoracic Society, May 2013.

Chapman D.G., Hoffman S, van der Velden J.L.J., Lahue K, Tully J, Tracy R.P., Worthen G.S., Poynter M.E., Janssen-Heininger Y.M.W., Irvin C.G. (2012) duffy antigen receptor for chemokines in a mouse model of allergic airways disease. American Thoracic Society, May 2013.

van der Velden J.L.J., Hoffman S, Tully J, Lundblad L.KA., Lahue K, Guala A, Irvin C.G., JanssenHeininger Y.M.W. (2012) Absence of c-Jun N-Terminal kinase 1 protects against HDM-induced pulmonary remodeling but not airway hyperesponsiveness. American Thoracic Society, May 2013.

Hoffman S, Aesif S, Lahue K, Tully J, Anathy V, Janssen-Heininger Y.M.W. Epithelial-specific overexpression of glutaredoxin-1 causes enhanced LPS-induced pulmonary inflammation, in association with decreased protein S-glutathionylation. American Thoracic Society, May 2012.

van der Velden J.L.J., Lundblad, Li S, Aliyeva M, Daphtary N, Hoffman S, Herath S.A.C., Lahue K, Janssen-Heininger Y.M.W. (2012) Airway remodeling in house dust mite-induced allergic airway disease. American Thoracic Society, May 2012.

Lundblad L.K.A., Li S, Aliyeva M, Daphtary N, van der Velden J.L.J., Hoffman S, Lahue K, Janssen-Heininger Y.M.W. (2012) Bronchoconstriction in mice allergic to house dust mite; a role for mast cells? American Thoracic Society, May 2012.

Hoffman S, Lahue K, Tully J, Ho Y.S., Irvin C.G., Lundblad L.K.A., Aliyeva M, Daphtary N, Janssen-Heininger Y.M.W. Genetic ablation of glutaredoxin-1 causes an enhanced resolution of airways hyperresponsiveness (AHR) in mice, in association with increases in protein Sglutathionylation. American Thoracic Society, May 2011. 


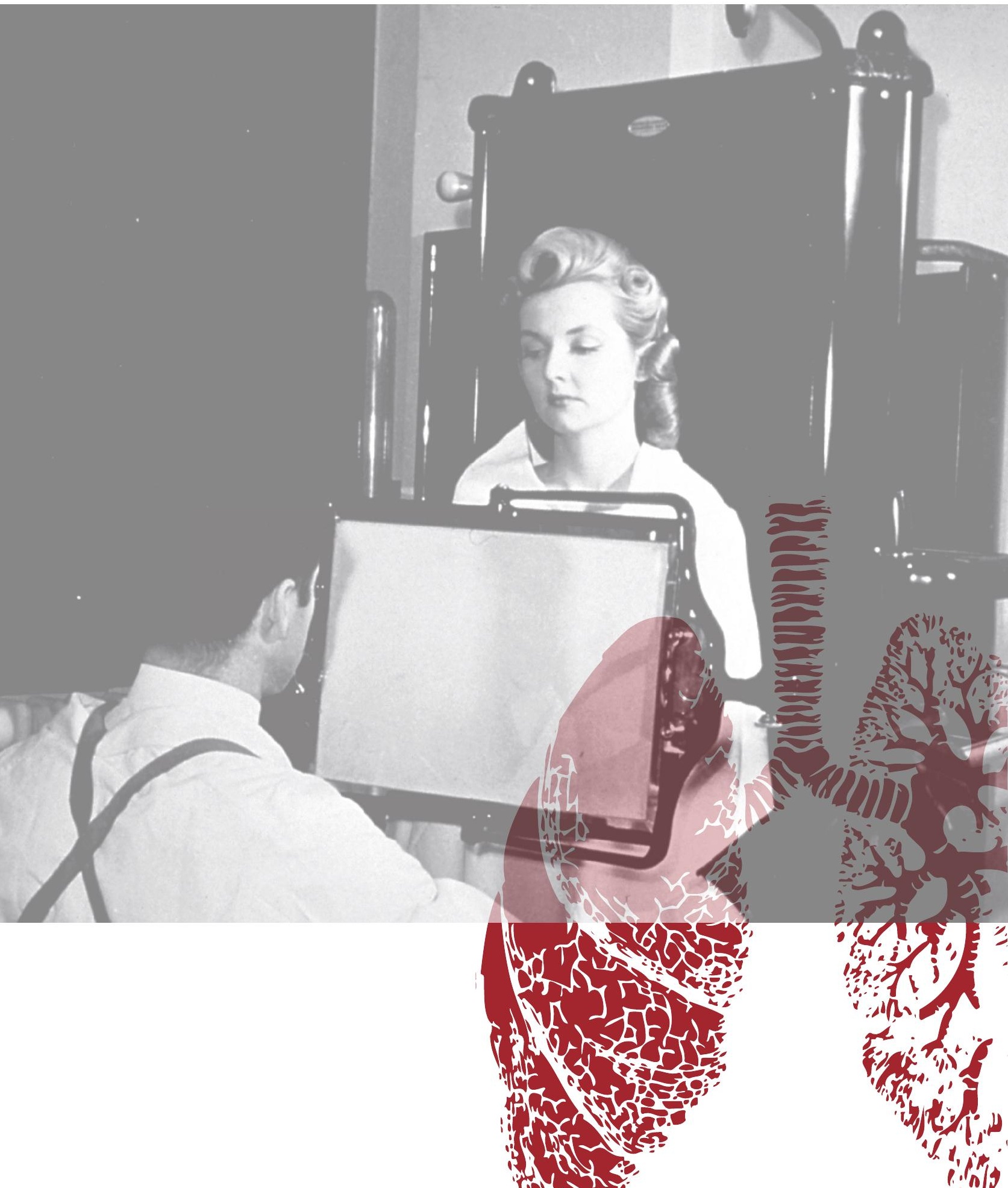

